\title{
Transport and nonequilibrium dynamics in 1d many-body systems with bosonic degrees of freedom
}

\author{
Dissertation \\ for the award of the degree \\ "Doctor rerum naturalium" (Dr.rer.nat.) \\ of the Georg-August-Universität Göttingen
}

within the doctoral program of the faculty of physics of the Georg-August University School of Science (GAUSS)

\author{
submitted by \\ Jan Felix Stolpp \\ from Frankfurt am Main
}

Göttingen, 2021 


\section{Thesis Committee:}

Prof. Dr. Fabian Heidrich-Meisner

Institut für Theoretische Physik, Georg-August-Universität Göttingen

Prof. Dr. Mathias Krüger

Institut für Theoretische Physik, Georg-August-Universität Göttingen

Prof. Dr. Christian Jooss

Institut für Materialphysik, Georg-August-Universität Göttingen

Members of the Examination Board:

Reviewer: Prof. Dr. Fabian Heidrich-Meisner

Institut für Theoretische Physik, Georg-August-Universität Göttingen

Second Reviewer: Prof. Dr. Eric Jeckelmann

Institut für Theoretische Physik, Leibniz Universität Hannover

\section{Further members of the Examination Board:}

Prof. Dr. Peter E. Blöchl

Institut für Theoretische Physik, Technische Universität Clausthal

Prof. Dr. Matthias Krüger

Institut für Theoretische Physik, Georg-August-Universität Göttingen

Prof. Dr. Stefan Mathias

I. Physikalisches Institut, Georg-August-Universität Göttingen

Prof. Dr. Peter K. Sollich

Institut für Theoretische Physik, Georg-August-Universität Göttingen

date of the oral examination: 12.03.2021 


\section{List of publications}

Parts of this thesis are published in peer reviewed journals and as preprints:

- S. Scherg, T. Kohlert, J. Herbrych, J. Stolpp, P. Bordia, U. Schneider, F. Heidrich-Meisner, I. Bloch, and M. Aidelsburger. Nonequilibrium mass transport in the 1D Fermi-Hubbard model. Phys. Rev. Lett., 121:130402, Sep 2018.

- D. Jansen, J. Stolpp, L. Vidmar, and F. Heidrich-Meisner. Eigenstate thermalization and quantum chaos in the Holstein polaron model. Phys. Rev. B, 99:155130, Apr 2019.

- J. Stolpp, J. Herbrych, F. Dorfner, E. Dagotto, and F. Heidrich-Meisner. Charge-density-wave melting in the one-dimensional Holstein model. Phys. Rev. B, 101:035134, Jan 2020.

- T. Köhler, J. Stolpp, and S. Paeckel. Efficient and flexible approach to simulate low-dimensional quantum lattice models with large local Hilbert spaces. SciPost Phys., 10:58, 2021.

- J. Stolpp, T. Köhler, S. R. Manmana, E. Jeckelmann, F. HeidrichMeisner, and S. Paeckel. Comparative study of state-of-the-art matrixproduct-state methods for lattice models with large local Hilbert spaces. arXiv:2011.07412, Nov 2020.

Additionally, the author contributed to another publication while working on this thesis:

- J. Stolpp, S.-S. Zhang, F. Heidrich-Meisner, and C. D. Batista. Large magnetic thermal conductivity induced by frustration in low-dimensional quantum magnets. Phys. Rev. B, 99:134413, Apr 2019. 


\section{Contents}

1 Introduction $\quad 5$

2 Electron-phonon coupling $\quad 12$

2.1 Phonons . . . . . . . . . . . . . . . . . . . 14

2.2 Phenomena resulting from electron-phonon interaction . . . . 16

3 Holstein model $\quad 19$

3.1 Limiting cases . . . . . . . . . . . . . . . . . . . 21

3.2 Phase diagram at half filling . . . . . . . . . . . . 23

4 Matrix-product-state methods $\quad 26$

4.1 Matrix product states . . . . . . . . . . . . . . 26

4.1.1 Entanglement entropy . . . . . . . . . . . . . . 29

4.1.2 Compressing a matrix product state . . . . . . . . 31

4.1.3 Matrix product operators . . . . . . . . . . . 32

4.1.4 U(1)-symmetric matrix product states . . . . . . . 32

4.2 Time evolution with matrix product states . . . . . . . . . . 34

4.3 Ground-state search with matrix product states . . . . . . . . 37

4.3.1 Two-site DMRG . . . . . . . . . . . . . . 38

4.3.2 Subspace expansion . . . . . . . . . . . . 40

5 Local basis optimization $\quad 42$

5.1 TEBD with local basis optimization . . . . . . . . . . . 44

5.2 Single-site ground-state DMRG with local basis optimization . 46

$\begin{array}{llr}6 & \text { Projected purification } & 49\end{array}$

7 Ground-state calculations in the Holstein model 53

7.1 Properties of the DMRG3S+LBO implementation . . . . . . . 54

7.2 Test against exact diagonalization . . . . . . . . . . . . . . 59

7.3 Benchmark of DMRG3S+LBO against other DMRG methods . . . . . . . . . . . . . . . 62 
7.4 Summary . . . . . . . . . . . . . . 70

8 Charge-density-wave melting in the Holstein model $\quad 74$

8.1 Bare charge-density-wave melting . . . . . . . . . . . 76

8.2 Dressed charge-density-wave melting . . . . . . . . . . . . . 82

8.3 Quenches from the CDW to the metallic phase . . . . . . . . 88

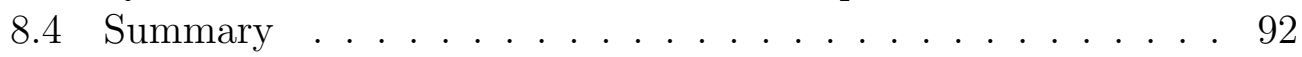

9 Thermalization in the Holstein model 94

9.1 Eigenstate thermalization hypothesis . . . . . . . . . . . . . 94

9.1.1 Quantum chaos . . . . . . . . . . . . 95

9.1 .2 ETH ansatz . . . . . . . . . . . . . . . 96

9.1.3 Exceptions from ETH _. . . . . . . . . 97

9.2 ETH test in the Holstein polaron model . . . . . . . . . . . 97

9.2.1 Quantum chaos indicators . . . . . . . . . . 98

9.2.2 Diagonal elements of observables . . . . . . . . . . . 101

9.2.3 Offdiagonal elements of observables . . . . . . . . . . 104

9.2.4 Variances of diagonal vs. offdiagonal matrix elements . 108

9.3 Summary . . . . . . . . . . . . . . . . . . . . 111

10 Sudden expansion in the 1d Fermi-Hubbard model 113

10.1 Earlier sudden expansion experiments in the Fermi- and Bose-

Hubbard model . . . . . . . . . . . . . . . . . . . . . . 115

10.1.1 Quantum distillation . . . . . . . . . . . 117

10.1.2 Probing quantum distillation in the 1d Bose-Hubbard model . . . . . . . . . . . . . . . . 118

10.2 Sudden expansion of a fermion cloud . . . . . . . . . . . . 119

10.2.1 Expansion with doublons in the initial state . . . . . 121

10.2.2 Expansion without doublons in the initial state . . . . 124

10.3 Summary . . . . . . . . . . . . . . . . . . . 128

11 Conclusion and outlook 130

A Charge-density-wave melting in the Holstein model: Finitesize dependence 


\section{Chapter 1}

\section{Introduction}

Atoms arranged in a periodic lattice structure are a common starting point for the description of solid-state materials. The atomic cores (the nucleus + the core electrons) form a periodic potential for the electrons. A fully rigid and perfectly periodic lattice structure leads to Bloch states for the electrons, under the condition that they are non-interacting. Although the assumptions made seem to be very strong approximations (the electrons definitively interact via screened Coulomb repulsion and the atoms will always have some movement for non-zero temperature), the resulting band theory is able to describe important phenomena like metals and band insulators [1].

The success of the non-interacting theory can be justified within FermiLiquid theory. Including weak short-range repulsive interactions between the electrons does not change the physics qualitatively. Important features, like the existence of a Fermi edge, carry over, while the non-interacting electrons have to be replaced by interacting quasi particles with a renormalized mass but otherwise identical quantum numbers as the bare electrons [2].

The assumption of a rigid lattice structure on the timescales of the electrons can be justified by noting that the mass of an atomic nucleus exceeds the electron mass by orders of magnitude (proton mass $\approx 1 \mathrm{u}$ vs. electron mass $\left.\approx 5 \times 10^{-4} \mathrm{u}[3]\right)$. The movements of the atomic cores is therefore slow compared to the electronic motion. The electronic state can instantaneously adapt to changes in the potential landscape caused by core movements. Formally, this can be derived by first splitting off the kinetic energy term for the cores from the full Hamiltonian to arrive at the Born-Oppenheimer Hamiltonian. Eigenstates of the Born-Oppenheimer Hamiltonian are coupled to each other through the kinetic term of the cores when the full Hamiltonian is considered. Ignoring these so called derivative couplings leads to the Born-Oppenheimer approximation (also referred to as adiabatic approximation) $[4,5]$. As we will see below, the notion that electrons do always adapt 
instantaneously to the potential landscape generated by the atomic cores can, however, break down.

In reality, lattice vibrations are always present at non-zero temperatures. In a quantum mechanical description, lattice excitations are referred to as phonons. The interaction between electrons and phonons plays a crucial role in understanding basic phenomena like the finite electric conductivity in metals [1]. Furthermore, strong electron-phonon coupling can lead to more striking phenomena. Prominent examples include the emergence of polarons and subsequent self trapping [6], the formation of charge-densitywave states [7], or BCS superconductivity [8].

In addition, electron-phonon coupling plays a crucial role as a relaxation channel after a system is taken far from equilibrium. Such situations come up in so called pump-probe experiments that have become a popular scheme in recent years to study solid-state materials $[9,10]$. These experiments bring a solid-state system out of equilibrium by hitting it with a strong laser pulse (pump pulse). After a variable waiting time, the sample is exposed to a second laser pulse (probe pulse) and the diffracted light of the second pulse is analysed. This experimental protocol allows for a time dependent study of excitation dynamics in the sample. In particular, laser-pulse durations on the femtosecond timescale allow for the direct observation of electron-relaxation dynamics, which opened the field of so called ultrafast dynamics in solids [9]. With this technology, many novel phenomena could be explored. Fascinating are the reported observation of photo-induced enhanced superconductivity [11], or the emergence of superconductivity in non-equilibrium above the critical temperature [12]. The melting of charge-density-wave order $[13,14]$ and the switching between different charge-density-wave patterns [15] were studied. Furthermore, metastable states can be accessed [16] and phase transitions between metallic and insulating states can be induced $[17,18]$.

To describe the dynamics of the experiments in theory, the full solid-state system is, in a first approximation, often treated as a collection of coupled sub-systems [10]. These are the electronic sub-system, the lattice degrees of freedom and possibly spin excitations. Specific modes in a selected subsystem can be targeted by tuning the frequency of the pump pulse. In many experiments, the pump pulse primarily couples to the electrons and excites them to a non-equilibrium state. Then the scattering between the electrons and their coupling to the lattice lets the system evolve to a steady state [10]. Understanding these competing relaxation channels is a task for theoreticians to comprehend the observations.

To approach this problem, it is helpful to resort to simple model systems. This ansatz allows to isolate certain aspects of more complicated solid-state systems, giving the opportunity to understand the influence of specific system 
parameters on a phenomenon without disguise by other effects. The bulk of this work is devoted to the study of exactly such a model system, namely the Holstein model of spinless fermions in one dimension [19,20]. The model comprises of non-interacting spinless fermions hopping on a tight binding chain. The fermions are locally coupled to harmonic oscillators. The harmonic oscillators at each site are decoupled from each other, thereby playing the role of an idealized Einstein phonon branch. Hence, direct electron-electron interaction or spin degrees of freedom are neglected. Further, phonons can only propagate through the system by being absorbed by a fermion at one site and then reemitted at another.

Despite its simplicity, the model hosts two phenomena that are typical for electron-phonon coupled systems, namely polaron formation [21] and, at half filling, a phase transition between a metallic Tomonaga-Luttinger liquid phase to an insulating charge-density-wave phase [22-24]. This makes it a popular platform in solid-state theory to explore these phenomena and to test different analytical and numerical techniques tailored for systems with electron-phonon coupling [22,24-38].

In this work, the Holstein model will be investigated by means of wavefunction based numerical techniques (exact diagonalization [39] and the densitymatrix renormalization group (DMRG) [40-42]). These require the Hilbert space of the problem to be of finite dimension, however, the local harmonic oscillators can in principle host arbitrarily many excitations, making already the Hilbert space of a single site infinite dimensional. This problem is resolved by a hard cutoff of the maximal local phonon number that has to be chosen appropriately depending on system parameters and the specific problem at hand. Still, the possibly large local Hilbert spaces pose a major challenge for the aforementioned numerical techniques and specialized algorithms have been developed to overcome this problem [28-30,37]. Here, two different ansatzes to improve the performance of density-matrix-renormalizationgroup methods will be introduced and used.

The first one is the so called local basis optimization (LBO) pioneered by Zhang et al. in Ref. [29] that aims to dynamically transform the local bosonic state space into a more efficient local basis that retains most of the state's information with a smaller local dimension. For this purpose, the singlesite reduced density matrix is computed and the state is locally transformed into its eigenbasis. The new local basis can then be truncated according to the eigenvalues of the single-site reduced density matrix, yielding an efficient approximation of the state.

Early efforts to incorporate LBO into ground-state DMRG methods con- 
sisted of calculating the optimized local basis on small systems and then using this basis for larger system sizes [43-45]. Further, DMRG-LBO algorithms were developed that calculated the local basis more dynamically [29, 46, 47]. In his thesis, F. Dorfner [48] developed a ground-state DMRG-LBO method by combining the ideas of Guo et al. [49] with the strictly single-site DMRG by Hubig et al. [50]. This method is tested here in detail. Especially, the inner workings and the interplay between algorithmic parameters with Hamiltonian parameters are analysed (see also Ref. [38]). Furthermore, the time-evolution DMRG-LBO ansatz developed by Brockt et al. [51-53] to investigate the dynamics of single electrons coupled to phonons, is extended in this work to half filled systems (see also Ref. [54]). In both the ground-state and timeevolution DMRG-LBO algorithms used here, the LBO is fully adaptive to the system size, system parameters, and the specific task at hand.

The second method is referred to as projected purification and was recently developed by Köhler, Paeckel, and the author [37]. In this method, the strategy is to utilize the well known performance gains from exploiting particle number conservations in the density-matrix renormalization group $[55,56]$. Since the number of phonons in the Holstein model is not conserved initially, the problem is projected to an enlarged Hilbert-space where a global $U(1)$ symmetry is restored. For this purpose, artificial phonon bath sites are introduced for every physical site, so that the combined number of phonons on every pair of physical and bath site is conserved.

To test the two methods, ground-state calculations are performed for the Holstein model at half filling and compared to the established pseudosite method [28] (see also Ref. [38]). For the comparison, the energy in the ground state as well as the displacement of the local oscillators is measured. Different parameter sets of the Holstein model are chosen, corresponding to distinct parts of the phase diagram. This way, a comprehensive overview of the methods performance in the different regions can be given.

Overall, all three methods are able to provide very precise results for every of the considered data points. The projected-purification method is conceptually quite simple and a two-site solver for the density-matrix renormalization group is used here, which makes handling the code very straight forward. In contrast, the local basis optimization introduces the number of optimized basis states as a new parameter in the code and a single-site solver with subspace expansion is used in the density-matrix renormalization group. This makes the method more delicate to converge. On the other hand, the local basis optimization yields a more compact representation of the ground states compared to the projected-purification, which becomes important close to the phase transition of the Holstein model. 
After testing the performance of the local basis optimization in a groundstate calculation, DMRG-LBO is applied to a time dependent problem (see also Ref. [54]). Motivated by the pump-probe experiments mentioned above, the ultra-short-time dynamics of charge-density-wave ordered states in the Holstein model is studied. Complementary to earlier work by Hashimoto and Ishihara [57], no direct interaction between electrons is present here and the emphasis is on the far-from-equilibrium regime. For this purpose three different initial states are considered that are time evolved at different points in the phase diagram. The first is a product state where every second site in the Holstein chain is occupied with an electron and no phonons are present initially; this one is termed bare charge-density-wave state. The second is a product state as well, but in this case every second site is occupied by a polaron. This one is referred to as dressed charge-density-wave state. As the last initial state, correlated ground states in the charge-density-wave phase of the Holstein model are considered.

The dynamics strongly depends on the parameters in the Hamiltonian and on the initial states. Nevertheless, a melting of the charge-density-wave order is found in all cases, as is expected in non-equilibrium. To track the melting, the charge-density-wave order parameter (staggered electron density) is followed as a function of time. In case of the bare charge-density-wave state, a transient self trapping effect can be observed for large electron-phonon couplings. This phenomenon results in a cascade like decay of the order parameter (a corresponding result was found in single-electron dynamics by Kloss et al. [58]). When the system is prepared in a correlated charge-density-wave ground state and quenched to the Tomonaga-Luttinger liquid phase, different timescales in the relaxation of the initially present electronic and phononic order can be detected. The electrons move through the lattice such that the order parameter decays. However, the phonons can only move by being absorbed by the electrons first and than emitted at a different location. This process happens on longer timescales than the electron hopping, explaining the persistent phononic order.

Complementary to the study of the short-time dynamics, one can ask whether and how closed quantum systems thermalize in the limit of very long times. A now well established theory for exactly this problem is the so called eigenstate thermalization hypothesis pioneered by Deutsch [59] and Srednicki [60]. One of its main statements is that thermalizing Hamiltonians share characteristics of random matrices. This further implies that expectation values of observables in states from the eigenbasis of the Hamiltonian are a 
smooth function of the energy of the respective states, only [61,62]. Many works that study the eigenstate thermalization hypothesis numerically focus on Hamiltonians that are relevant for quantum gas experiments (Hubbard models or spin systems) [63-66]. The systems studied in these experiments are close to being isolated and, therefore, thermalization and its breakdown in integrable systems can be tested directly [67-69].

Here, thermalization is investigated in the Holstein model as an example for a more solid-state motivated system (see also Ref. [70]). Only a single fermion in the chain is considered, making the coupling energy between electrons and phonons, that breaks the integrability of the system, an intensive term in the Hamiltonian. Both, the structure of the energy spectrum and observables are analysed by means of exact diagonalization of the Hamiltonian for small systems with up to $L=8$ sites and a maximum of $M_{\mathrm{ph}}=3$ phonons per site. Comparing the results to predictions of the eigenstate thermalization hypothesis yields a very good agreement. The findings suggest thermalizing behavior for fixed $M_{\mathrm{ph}}$ in the limit of large systems $(L \rightarrow \infty)$. These results are non trivial, since there is only a single electron in the system and, therefore, the integrability breaking electron-phonon energy is not extensive but of order $\mathcal{O}(1)[71-76]$.

The subject of the last part of this thesis is somewhat detached from the rest. There, mass transport in the 1d Fermi-Hubbard model is studied by means of a quantum gas experiment $[77,78]$ with additional support from time-dependent DMRG simulations. The model describes electrons in a tight-binding chain with onsite interactions, while a coupling to the lattice degrees of freedom is neglected, in contrast to the Holstein model.

The experimental study presented here (see also Ref. [79]) is motivated by preceding investigations of sudden expansion in the Bose-Hubbard model $[80,81]$. The concept of these experiments is to prepare a cloud of particles in the center of an otherwise empty lattice. Upon release, the particles tunnel to the empty part. The expansion dynamics of the particle cloud is strongly dependent on the interaction strength between the particles and the initial state. In particular, a reduction of the expansion velocity for intermediate interaction strengths was found in the 1d Bose-Hubbard model, when only singly occupied sites are present in the initial state [80]. Furthermore, signatures of quantum distillation [80-84] were detected in case the initial state consists of a mixture of singly and multi occupied sites [80,81]. This phenomenon features an accumulation of multi occupations in the center while single occupations escape.

In the experimental study presented here, clouds of fermions are prepared 
in local Fock states. If doubly occupied sites are present in the initial state, a large interaction leads to the dynamical formation of a stable high density core, while a low density background expands. This is clear signature of fermionic quantum distillation. If all the sites in the initial cloud are only singly occupied, double occupations are generated dynamically. Similar to the bosonic case, the expansion velocity is then reduced for intermediate interaction strengths. However, this reduction is far less pronounced here, since two is the highest occupation possible in case of fermions.

The results of the quantum gas experiment are analysed with support of numerical time-dependent DMRG calculations. These calculations show that the efficiency of the quantum distillation mechanism is reduced by the existence of completely unoccupied sites in the initial state. Furthermore, the reduced expansion velocity in case of only single occupations in the initial state can be traced back to converting kinetic energy into interaction energy. This mechanism is found both in the fermionic and bosonic case.

This work is structured as follows. In Ch. 2, the origin of electron-phonon coupling and resulting phenomena in solid-state systems are discussed. The main study object of this thesis, the Holstein model of spinless fermions is introduced in Ch. 3. Chapter 4 contains a recapitulation of matrix-productstate methods for time evolution and ground-state calculations, before two specialized ansatzes to optimize matrix-product-state methods for electronphonon systems, the local basis optimization and the projected purification, are introduced in Ch. 5 and Ch. 6, respectively. These two methods are used in Ch. 7 to calculate ground states of the half-filled Holstein model. The local basis optimization is again employed for time evolution in Ch. 8 to study charge-density-wave melting in the Holstein model. In Ch. 9, the eigenstate thermalization hypothesis is put to the test in the same model but for a single itinerant charge carrier. The focus shifts from electronphonon coupling to dominating electron-electron interaction in Ch. 10 where the sudden expansion of a fermion cloud in the 1d Fermi-Hubbard model is discussed. This thesis ends with a conclusion and outlook in Ch. 11. 


\section{Chapter 2}

\section{Electron-phonon coupling}

The full Hamiltonian to describe a solid-state crystal comprises terms for the movement of the atomic nuclei and all the electrons as well as terms for the interaction between these constituents. However, in most materials, for every nucleus, there are some electrons that are always tightly bound to it. These electrons do not directly take part in the binding between the atoms and, therefore, play a subordinary role for the electronic properties of the solid. These electrons are the so called core electrons, and in the description of solids the atomic nuclei and the core electrons are often merged to form the atomic cores. The rest of the electrons, the so called valence electrons, are kept explicitly in the description. This leads to the Hamiltonian $[1,85]$ :

$$
\begin{aligned}
H= & \sum_{i} \frac{\mathbf{P}_{i}^{2}}{2 M_{i}}+\sum_{j} \frac{\mathbf{p}_{j}^{2}}{2 m_{\mathrm{e}}} \\
& +\frac{1}{2} \sum_{\substack{i, i^{\prime} \\
i \neq i^{\prime}}} V_{1}\left(\mathbf{R}_{i}, \mathbf{R}_{i^{\prime}}\right)+\frac{1}{2} \sum_{\substack{j, j^{\prime} \\
j \neq j^{\prime}}} V_{2}\left(\mathbf{r}_{j}, \mathbf{r}_{j^{\prime}}\right)+\sum_{i, j} V_{3}\left(\mathbf{R}_{i}, \mathbf{r}_{j}\right),
\end{aligned}
$$

where $\mathbf{R}_{i}$ and $\mathbf{P}_{i}$ are the position and momentum operators of the atomic cores and $\mathbf{r}_{j}$ and $\mathbf{p}_{j}$ are the position and momentum operators of the valence electrons. $M_{i}$ are the masses of the atomic cores and $m_{\mathrm{e}}$ is the electron mass. The last three terms describe the interaction between the cores, the electrons, and the cores with the electrons, respectively.

The full Hamiltonian Eq. (2.1) can be reduced to the so called BornOppenheimer Hamiltonian by removing the kinetic energy term for the atomic cores $[4,5]$ :

$$
H_{\mathrm{BO}}=\sum_{j} \frac{\mathbf{p}_{j}^{2}}{2 m_{\mathrm{e}}}+\frac{1}{2} \sum_{\substack{i, i^{\prime} \\ i \neq i^{\prime}}} V_{1}\left(\mathbf{R}_{i}, \mathbf{R}_{i^{\prime}}\right)+\frac{1}{2} \sum_{\substack{j, j^{\prime} \\ j \neq j^{\prime}}} V_{2}\left(\mathbf{r}_{j}, \mathbf{r}_{j^{\prime}}\right)+\sum_{i, j} V_{3}\left(\mathbf{R}_{i}, \mathbf{r}_{j}\right)
$$


The Born-Oppenheimer Hamiltonian is diagonal in the position basis of the atomic cores. For a given spatial configuration of the atomic cores one is, therefore, left with the electronic problem only.

The electronic problem alone is already a very demanding many-body problem and in the most general form there is no hope of solving it. Nevertheless, let me assume that I got a solution for the electron problem somehow. A generic wave function in position space is:

$$
\psi(\overrightarrow{\mathbf{r}}, \overrightarrow{\mathbf{R}})=\langle\overrightarrow{\mathbf{r}}, \overrightarrow{\mathbf{R}} \mid \psi\rangle,
$$

where $\overrightarrow{\mathbf{r}}=\left(\mathbf{r}_{1}, \ldots \mathbf{r}_{N}\right)$ and $\overrightarrow{\mathbf{R}}=\left(\mathbf{R}_{1}, \ldots \mathbf{R}_{M}\right)$. The state $|\overrightarrow{\mathbf{r}}, \overrightarrow{\mathbf{R}}\rangle$ is a basis state in the position basis and can also be written as a product of the electronic and core part:

$$
|\overrightarrow{\mathbf{r}}, \overrightarrow{\mathbf{R}}\rangle=|\overrightarrow{\mathbf{r}}\rangle|\overrightarrow{\mathbf{R}}\rangle
$$

Since the Born-Oppenheimer Hamiltonian is diagonal in the position basis of the atomic cores, its eigenstates can be written as $|n(\overrightarrow{\mathbf{R}})\rangle|\overrightarrow{\mathbf{R}}\rangle$ such that $[5]$ :

$$
H_{\mathrm{BO}}|n(\overrightarrow{\mathbf{R}})\rangle|\overrightarrow{\mathbf{R}}\rangle=\epsilon_{n}(\overrightarrow{\mathbf{R}})|n(\overrightarrow{\mathbf{R}})\rangle|\overrightarrow{\mathbf{R}}\rangle
$$

For fixed $n$ the energies $\epsilon_{n}(\overrightarrow{\mathbf{R}})$ form surfaces in $\overrightarrow{\mathbf{R}}$-space, the so called BornOppenheimer surfaces.

One can now write the full Hamiltonian, Eq. (2.1), in the eigenbasis of the Born-Oppenheimer Hamiltonian, Eq. (2.5). In general, the kinetic term for the atomic cores in Eq. (2.1) leads to a coupling between different basis states, the so called derivative couplings [5]. However, if the different electronic eigenstates of the Born-Oppenheimer Hamiltonian, Eq. (2.2), are well separated for a given core configuration, the coupling between the electronic eigenstates will be very weak. In this situation, the ansatz states from Eq. (2.5) are close to being eigenstates of the full Hamiltonian, Eq. (2.1). If one prepares the electrons in a specific eigenstate $|n(\overrightarrow{\mathbf{R}})\rangle$ these will stay in the $n$th eigenstates, even if one slowly deforms the core configuration. The situation where one ignores the electron-lattice coupling through the kinetic energy term of the cores is also referred to as the adiabatic approximation [5].

If, however, the electronic levels come closer together, the situation is different. A transfer between different electronic levels is then possible by exchanging energy with the atomic core configuration. The states in Eq. (2.5) will then no longer resemble the eigenstates of the full system. In particular, the derivative couplings diverge in the vicinity of points in $\overrightarrow{\mathbf{R}}$-space where Born-Oppenheimer surfaces touch [5]. In this case, electrons and atomic cores have to be treated on equal footing. 


\subsection{Phonons}

For a quantum-mechanical description of the atomic cores, it is convenient to use the language of phonons. The starting point for this description is a collection of $N$ atomic cores, arranged in a periodic lattice structure, that are described classically. The cores are coupled with each other in such a way, that a deviation of the equilibrium distance between the cores leads to a linear restoring force. This approximation is certainly valid for small deviations from the equilibrium distances. One ends up with a system of $N$ coupled harmonic oscillators. In $d$ spatial dimensions the description leads to $d N$ oscillator modes [1].

The dispersion relation of the $d N$ oscillator modes can be grouped into different branches. As long as all atomic cores have the same mass and the coupling between the cores along all spatial directions is homogeneous, only acoustic branches appear. The dispersion relation of these acoustic branches vanishes linearly when the wave vector goes to zero:

$$
\omega_{\mathrm{A}}(\mathbf{k}) \propto|\mathbf{k}| \text { for }|\mathbf{k}| \rightarrow 0 .
$$

As soon as atomic cores with different masses are present in the system or the coupling between the cores is not homogeneous, so called optical branches show up. The dispersion of these optical branches does not vanish for long wavelengths:

$$
\omega_{\mathrm{O}}(\mathbf{k}) \rightarrow \omega_{0} \text { for }|\mathbf{k}| \rightarrow 0 .
$$

A sketch of a typical phonon dispersion in 1D with an acoustic and optical branch is shown in Fig. 2.1 [1].

Upon quantisation of the problem, each of the $d N$ classical oscillator modes can be treated as an independent quantum oscillator. These quantum oscillators can be in certain excitation states specified by the occupation numbers $n_{\mathbf{k} b}$ and their energy is:

$$
E_{\mathbf{k} b}=\left(n_{\mathbf{k} b}+\frac{1}{2}\right) \omega_{b}(\mathbf{k})
$$

where $b$ is a branch index and $\omega_{b}(\mathbf{k})$ is the dispersion from the classical description. Analogous to the description of excitations of the light field as photons, the excitations of the oscillator modes in a solid are called phonons [1].

Two rather radical approximations are frequently used when dealing with phonons, so I will quickly recap them here. The first one was used by Debye to describe the specific heat of a solid [86]. In the Debye description in $d$ 


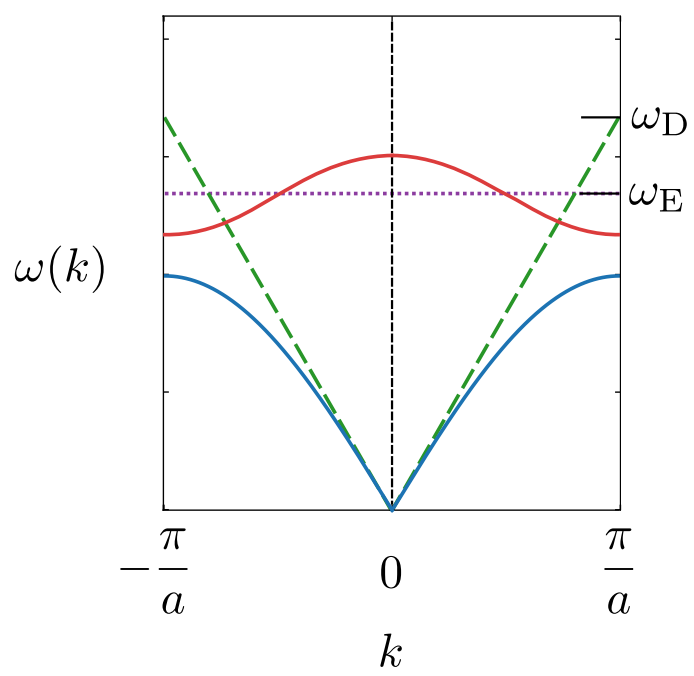

Figure 2.1: Sketch of a phonon dispersion in 1D with an acoustic branch (blue) and an optical branch (red). The dashed green line represents the Debye approximation for the acoustic branch and the purple dotted line represents the Einstein approximation of the optical branch, where $\omega_{\mathrm{D}}$ is the Debye frequency and $\omega_{\mathrm{E}}$ is the Einstein frequency.

dimensions, all phonon branches are replaced by $d$ phonon branches with the same linear dispersion (see Fig. 2.1):

$$
\omega(\mathbf{k})=c|\mathbf{k}|,
$$

where $c$ is a constant with units of a velocity. In addition, one defines a maximum wave vector $k_{\mathrm{D}}$ such that every branch contains exactly $N$ phonon modes. This cutoff replaces the restriction to a single Brillouine zone. The volume of a single wave vector in $k$-space is $(2 \pi)^{d} / V$, where $V$ is the volume of the solid. $N$ times this volume has to be equal the volume of a $d$ dimensional sphere in $k$-space with radius $k_{\mathrm{D}}$. Therefore, $k_{\mathrm{D}}$ is defined through the relation:

$$
n=\frac{N}{V}=\frac{V_{d}}{(2 \pi)^{d}} k_{\mathrm{D}}{ }^{d},
$$

where $V_{d}$ is the volume of the unit sphere in $d$ dimensions. From $k_{\mathrm{D}}$, one can define the Debye frequency $\omega_{\mathrm{D}}$ which is a measure for the highest frequency in the solid:

$$
\omega_{\mathrm{D}}=c k_{\mathrm{D}}
$$

Despite being a very crude approximation, the Debye model is commonly used. By choosing as the constant $c$ the average of the phase velocity in the 
long-wavelength limit of the acoustic branches, the model can, for example, provide reliable results for low temperatures [1].

The second approximation that I want to mention goes back to Einstein [87]. One envisions the solid as consisting of independent harmonic oscillators whose frequency is $\omega_{\mathrm{E}}$. The dispersion relation is therefore just a constant (see Fig. 2.1):

$$
\omega(\mathbf{k})=\omega_{\mathrm{E}} .
$$

Such a dispersion can be used to approximate the optical branches of the phonon dispersion.

Electron-phonon interaction can be categorized into being short or long range. In ionic and polar materials the potential for a charge carrier is formed by the dipole fields of the constituents. Changing the distance between the ions of a dipole alters the coulomb potential seen by a charge carrier, leading to a long range coupling. A short range coupling results from a dependence of the potential seen locally by a charge carrier on the strain at the respective location in the material [6]. This scenario is described by the Holstein model where the on-site energy level depends linearly on the displacement of a harmonic oscillator at the site $[19,20]$ (see also Ch. 3).

\subsection{Phenomena resulting from electron-phonon interaction}

The phonon picture is particularly helpful to describe the interaction between electrons and lattice degrees of freedom. Electrons moving through the lattice can bump into an atomic core and emit or absorb phonons in the process . These processes lead to the relaxation of electrical currents in metals and are the standard explanation for the temperature dependence of the resistivity [1]. In the following, other prominent examples of phenomena resulting from electron-phonon interactions are introduced.

The original notion of a polaron, going pack to a paper by Landau [88], is that of an electron bound to a potential indentation. Such an indentation can be caused by the electron itself attracting the surrounding polar lattice ions and thereby deforming the lattice. The movement of the electron is, therefore, hindered substantially since the deformation of the lattice has to travel simultaneously. The response of the lattice to the electron attraction, however, is rather slow on timescales of the electron movements. The result is a so called self trapping effect with a large effective mass of the polaron compared to the bare electron mass. This situation corresponds to the strong coupling limit between electrons and phonons [6]. 
In a broader sense, polarons are electrons spatially correlated to phonon excitations. If the coupling between electrons and phonons is weak this will not impede the electron movement too much. Rather, the result is a moderate increase of the effective mass [6].

Furthermore, a categorization of polarons can be made depending on the extend of the induced lattice deformations. In the case of large polarons the the lattice deformations are spread over several sites while for small polarons these deformations are primarily confined to the location of the electron.

In addition to the change of the effective mass of the electrons, the deformation of the lattice can have an effect on the interaction between the electrons. Bi-polarons are formed if it becomes favorable for two charge carriers to join forces and occupy the same self-trapping potential well [6]. On the other end of the spectrum, a repulsive interaction between polarons can lead to charge density waves. Such a situation is found in the Holstein model of spinless fermions [22] as will be discussed later.

Electron-phonon coupling plays a key role in conventional BCS superconductors [89]. Cooper showed that a Fermi liquid is unstable against attractive interactions between electrons at the Fermi edge [90]. Such an interaction leads to the formation of bound states of two electrons with opposite momentum and spin, the so called Cooper pairs. The necessary attractive interaction is a result of the interaction between electrons and lattice as shown by Bardeen, Cooper, and Schrieffer $[8,85]$.

The superconducting gap in this theory takes the form [89]:

$$
\Delta \approx \hbar \omega_{c} \mathrm{e}^{-1 /\left(N_{0} V\right)},
$$

where $\omega_{c}$ is a cutoff frequency, $N_{0}$ is the density of states at the fermi edge, and $V$ is the attractive interaction strength between the electrons. As an estimate for the cutoff frequency one can take the Debye frequency which in turn depends on the mass of the atoms in the crystal as $\omega_{\mathrm{D}} \propto 1 / \sqrt{M}[1]$. This leads to the so called isotope effect. If one changes the isotope of an element superconductor, this changes the size of the gap and therefore the critical temperature as $T_{\mathrm{C}} \propto 1 / \sqrt{M}$. A necessary condition is of course that all other parameters in Eq. (2.13) remain unchanged. The prediction of an isotope effect in case electron-lattice interactions play a role in superconductivity by Fröhlich [91, 92] and the subsequent measurement of such an effect [93, 94] paved the way towards the development of the BCS-theory.

The role of electron-phonon coupling in the context of high- $T_{\mathrm{c}}$ superconductivity is less clear. The most widespread working hypothesis is that spin excitations are responsible for the pairing mechanism at least in the cuprate [95] and iron based superconductors [96]. The role of electron-phonon coupling in these compounds is, therefore, less prominent. 


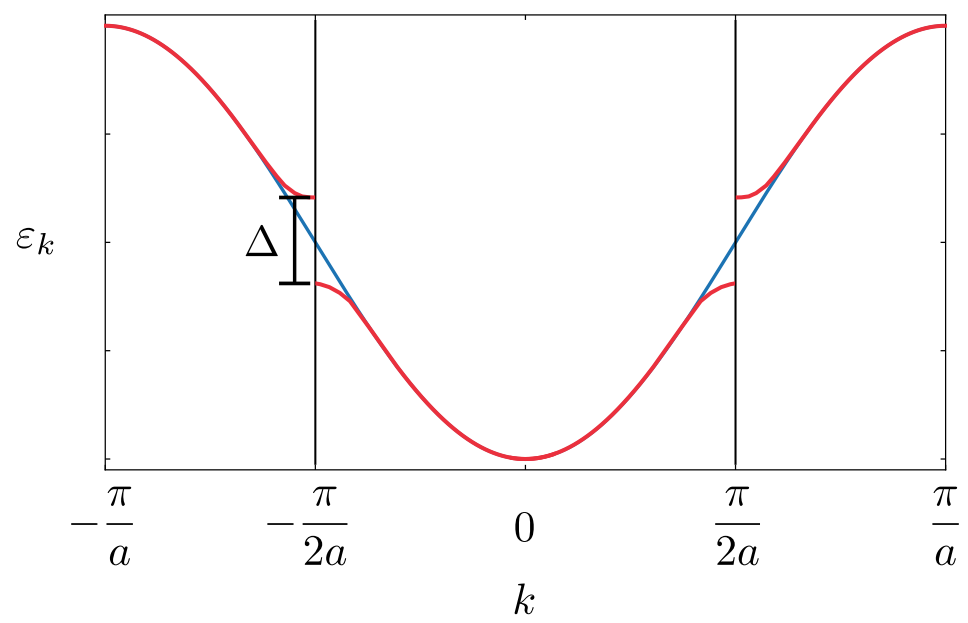

Figure 2.2: Sketch of the gap opening in a electronic dispersion as a consequence of a periodic lattice distortion that doubles the unit cell. If the band is half-filled, the electrons close to the Fermi edge lose energy because of the gap opening, resulting in a band insulator (Peierls instability).

In one-dimensional systems exists a particular phenomenon associated with electron-phonon coupling. It was pointed out by Peierls, that partially filled bands in a one-dimensional conductor are unstable towards periodic lattice distortions [7,97]. Consider such a partially filled band (cf. Fig 2.2). The Fermi surface consists of the two wave vectors $\pm k_{\mathrm{F}}$.

Now also consider an acoustic phonon branch. If the lattice is periodically distorted this leads to an enlargement of the unit cell and consequently the Brillouin zone shrinks. If the wave vector of this periodic distortion is $k_{\mathrm{ph}}=$ $2 k_{\mathrm{F}}$ the Fermi surface falls on the boundary of the new Brillouin zone. The presence of the periodic potential leads to the well known gap opening in the electronic spectrum at the edge of the Brillouin zone [1].

As long as electronic states above and below the newly opened gap are occupied the total energy of the electronic system does not change. If $k_{\mathrm{ph}}=$ $2 k_{\mathrm{F}}$, however, only the states below the gap are occupied and the electronic system lowers its energy. The state with the opened gap and periodic lattice distortions will be the ground state of the combined electron-phonon system if the energy required to distort the lattice is less than the energy gain of the electronic system. In Ref. [98] it is argued that this is always the case for small static displacements. 


\section{Chapter 3}

\section{Holstein model}

In his seminal work from 1959, Holstein develops a model for a linear chain of identical diatomic molecules that is now commonly known as the Holstein model $[19,20]$. The center of mass and the orientation in space of all the molecules is fixed. What is left are the vibrational modes of the molecules. If one assumes a square potential for the relative displacement of the two atoms in a molecule from their rest position one ends up with a collection of uncoupled harmonic oscillators. Their Hamiltonian can be diagonalized in the occupation number basis of the harmonic oscillators and one ends up with:

$$
H_{\mathrm{ph}}=\omega_{0} \sum_{l=1}^{L} b_{l}^{\dagger} b_{l}
$$

where the $b_{l}\left[b_{l}^{\dagger}\right]$ are bosonic annihilation [creation] operators, $\omega_{0}$ is the frequency of the oscillators, and $L$ is the number of molecules (length of the chain). The model, therefore, features a single branch of Einstein phonons.

Next, one introduces electrons that can move in this chain of molecules. One chooses a tight binding ansatz for the electrons such that the electronic state is a superposition of states that are localized on specific molecules. For simplicity I will consider noninteracting spinless electrons here. In his original paper $[19,20]$ Holstein considers just a single electron in the lattice so the Hamiltonian in second quantisation takes the same form as the one for spinless fermions:

$$
H_{\mathrm{el}}=-t_{0} \sum_{l=1}^{L}\left(c_{l}^{\dagger} c_{l+1}+c_{l+1}^{\dagger} c_{l}\right),
$$

where the $c_{l}\left[c_{l}^{\dagger}\right]$ are fermionic annihilation [creation] operators and $t_{0}$ is the 


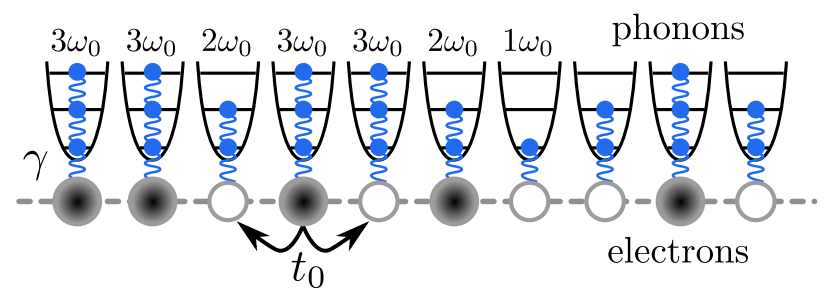

Figure 3.1: Sketch of the different terms in the Holstein Hamiltonian Eq. (3.4). Electrons hop from site to site with a hopping amplitude $t_{0}$. Every site has a harmonic oscillator attached to it with a frequency $\omega_{0}$. The excitations of the harmonic oscillators constitute an Einstein phonon branch. The density of the electrons at the sites couples to the displacement of the oscillators with a strength $\gamma$. Figure from Ref. [54]. (c) 2020 American Physical Society

hopping matrix element. In this form I assume periodic boundary conditions and identify $c_{L+1}$ as $c_{1}$.

The last ingredient of the model is an interaction between the electrons and the vibrations of the molecules. The basic consideration is that deformations of the lattice can change the energy levels of the electrons. In this case, one assumes a linear dependence of the electron level at a specific molecule on the relative displacement $X_{l}$ from the rest distance of the atoms in the molecule. The electron level is lowered when the atoms increase their relative distance:

$$
H_{\mathrm{el}-\mathrm{ph}}=-\gamma \sum_{l=1}^{L} n_{l}\left(b_{l}^{\dagger}+b_{l}\right)
$$

where $n_{l}=c_{l}^{\dagger} c_{l}$ and $\left(b_{l}^{\dagger}+b_{l}\right) \propto X_{l}$. In the following, I will refer to $\left(b_{l}^{\dagger}+b_{l}\right)$ as the displacement, although it is only proportional to the operator $X_{l}$.

The full Hamiltonian of the Holstein model then reads (see also Fig. 3.1):

$$
H_{\mathrm{Hol}}=H_{\mathrm{el}}+H_{\mathrm{ph}}+H_{\mathrm{el}-\mathrm{ph}} .
$$

The Hamiltonian implies that the hopping matrix element $t_{0}$ does not depend on the vibrational state of the molecules involved in the hopping. Importantly, the number of fermions $N=\sum_{l=1}^{L}\left\langle n_{l}\right\rangle$ is conserved but due to $H_{\mathrm{el}-\mathrm{ph}}$, Eq. (3.3), the number of phonons $N_{\mathrm{ph}}=\sum_{l=1}^{L}\left\langle b_{l}^{\dagger} b_{l}\right\rangle$ is not.

It is evident, that the Holstein model with its many simplifications is far from describing the dynamics in a real material. Nevertheless, it hosts 
a wealth of interesting physics typical for electron-phonon coupled systems $[21,22]$. Polarons with different characteristics can be found in the model, ranging from weak to strong dressing and from spread out large polarons to small polarons depending on the ratios of hopping $t_{0}$, phonon frequency $\omega_{0}$ and coupling $\gamma[21]$. If the density of electrons in the system becomes considerable, the phonons can mediate an effective interaction between the charge carriers. At half filling, this leads to a quantum phase transition between a metallic Tomonaga-Luttinger liquid phase and an insulating charge-densitywave phase [22-24].

\subsection{Limiting cases}

The main focus of this work is on the case near half filling. To get a better feeling of the model, it is helpful to consider the atomic limit $t_{0}=0$ of uncoupled molecules. The Hamiltonian of the molecule at site $l$ reads:

$$
h_{\text {atomic }, l}=\omega_{0} b_{l}^{\dagger} b_{l}-\gamma n_{l}\left(b_{l}^{\dagger}+b_{l}\right) .
$$

In the trivial case when no electron is at site $l$, the Hamiltonian reduces further to just the phonon part. In this case, the eigenstates can be written as:

$$
\frac{1}{\sqrt{n !}}\left(b_{l}^{\dagger}\right)^{n}|\emptyset\rangle_{\mathrm{el}, l}|\emptyset\rangle_{\mathrm{ph}, l}
$$

and the spectrum is equally spaced with spacing $\omega_{0}$. Here $|\emptyset\rangle_{\mathrm{el}, l}$ and $|\emptyset\rangle_{\mathrm{ph}, l}$ are the respective electron and phonon vacuum states at site $l$.

If there is an electron present at site $l$, the second term in Eq. (3.5) has to be considered and the local Hamiltonian reads:

$$
h_{\mathrm{atomic}, l}|1\rangle_{\mathrm{el}, l}=\left(\omega_{0} b_{l}^{\dagger} b_{l}-\gamma\left(b_{l}^{\dagger}+b_{l}\right)\right)|1\rangle_{\mathrm{el}, l},
$$

where $|1\rangle_{\mathrm{el}, l}=c_{l}^{\dagger}|\emptyset\rangle_{\mathrm{el}, l}$. The phononic part can be diagonalized by a LangFirsov transformation [99]:

$$
\tilde{b}_{l}^{\dagger}:=b_{l}^{\dagger}-\frac{\gamma}{\omega_{0}}
$$

which transforms Eq. (3.7) into:

$$
h_{\text {atomic }, l}|1\rangle_{\mathrm{el}, l}=\left(\omega_{0} \tilde{b}_{l}^{\dagger} \tilde{b}_{l}-\frac{\gamma^{2}}{\omega_{0}}\right)|1\rangle_{\mathrm{el}, l} .
$$


The ground state can be found by demanding that $\tilde{b}_{l}|1\rangle_{\mathrm{el}, l} \mid$ g.s. $\rangle_{\mathrm{ph}, l}=0$. In terms of the original phonon operators, it has the form of a coherent state:

$$
\left.|1\rangle_{\mathrm{el}, l} \mid \text { g.s. }\right\rangle_{\mathrm{ph}, l}=\mathrm{e}^{-\frac{\gamma^{2}}{2 \omega_{0}^{2}}} \mathrm{e}^{\frac{\gamma}{\omega_{0}} b_{l}^{\dagger}}|1\rangle_{\mathrm{el}, l}|\emptyset\rangle_{\mathrm{ph}, l} .
$$

The ground-state energy is:

$$
\epsilon_{0}=-\frac{\gamma^{2}}{\omega_{0}}=-\epsilon_{b}
$$

where $\epsilon_{b}$ is referred to as the polaron binding energy. The physical interpretation of the Lang-Firsov transformation is that the rest position of the harmonic oscillator is shifted by the presence of the electron.

The degenerate ground states of the full system are:

$$
\left.\mid \text { g.s. }\left(\left\{l_{\text {occ }}\right\}\right)\right\rangle=\mathrm{e}^{-\frac{N \gamma^{2}}{2 \omega_{0}^{2}}}\left[\prod_{l \in\left\{l_{\mathrm{occ}}\right\}} \mathrm{e}^{\frac{\gamma}{\omega_{0}} b_{l}^{\dagger}} c_{l}^{\dagger}\right]|\emptyset\rangle_{\mathrm{el}}|\emptyset\rangle_{\mathrm{ph}},
$$

where $N$ is the number of electrons and $\left\{l_{\mathrm{occ}}\right\}$ is the set of sites that are occupied by an electron. A different ground states can be generated by choosing a different set $\left\{l_{\mathrm{occ}}\right\} .|\emptyset\rangle_{\mathrm{el}}$ and $|\emptyset\rangle_{\mathrm{ph}}$ are the respective electron and phonon vacuum states of the full system.

The ground states Eq. (3.12) span a subspace of the full Hilbert space, that can be the starting point for a perturbative treatment of the Hamiltonian Eq. (3.4) in the small $t_{0}$ limit [22]. The unperturbed Hamiltonian is taken to be:

$$
H_{0}=H_{\mathrm{ph}}+H_{\mathrm{el}-\mathrm{ph}}=\omega_{0} \sum_{l=1}^{L} b_{l}^{\dagger} b_{l}-\gamma \sum_{l=1}^{L} n_{l}\left(b_{l}^{\dagger}+b_{l}\right),
$$

and the perturbation is:

$$
H_{1}=H_{\mathrm{el}}=-t_{0} \sum_{l=1}^{L}\left(c_{l}^{\dagger} c_{l+1}+c_{l+1}^{\dagger} c_{l}\right) .
$$

Consider two orthogonal ground states of the form Eq. (3.12) that differ only by the occupation of the two neighboring sites $l$ and $l+1$. In the first state site $l$ is occupied by a polaron and site $l+1$ is empty and in the second state the occupations of the two sites are swapped. I denote the first state as $\mid$ g.s. $\left.\left(\left\{\ldots, 1_{l}, 0_{l+1}, \ldots\right\}\right)\right\rangle$ and the second state as $\mid$ g.s. $\left.\left(\left\{\ldots, 0_{l}, 1_{l+1}, \ldots\right\}\right)\right\rangle$. In first order perturbation theory one obtains a polaron hopping matrix element:

$$
\left\langle\text { g.s. }\left(\left\{\ldots, 1_{l}, 0_{l+1}, \ldots\right\}\right)\left|H_{1}\right| \text { g.s. }\left(\left\{\ldots, 0_{l}, 1_{l+1}, \ldots\right\}\right)\right\rangle=-t_{0} \mathrm{e}^{-\frac{\gamma^{2}}{\omega_{0}^{2}}}=-\tilde{t}_{0},
$$


hence a narrow polaron band to first order.

To second order, two processes have to be considered. An electron can hop to a neighboring site and then back again. This process is of course only possible if the neighboring site is empty and, therefore, leads to a nearestneighbor repulsion. The second process is the hopping of a polaron to the next to nearest neighbor. In this case the process is only possible if the site in between is empty. The corresponding matrix elements are given by [22]:

$$
\begin{aligned}
& \tilde{V}=2 \frac{\tilde{t}_{0}^{2}}{\omega_{0}} \int_{0}^{\frac{\gamma^{2}}{2 \omega_{0}^{2}}} \mathrm{~d} g \frac{\mathrm{e}^{4 g}-1}{g}, \\
& \tilde{t}_{2}=\frac{\tilde{t}_{0}^{2}}{\omega_{0}} \int_{0}^{\frac{\gamma^{2}}{2 \omega_{0}^{2}}} \mathrm{~d} g \frac{\mathrm{e}^{2 g}-1}{g} .
\end{aligned}
$$

The resulting effective Hamiltonian is the one of repulsively interacting spinless fermions. For weak interactions, the model is in a metallic TomonagaLuttinger liquid phase. If the repulsive interaction is strong enough, the fermions do not want to reside on neighboring sites anymore and order themselves in a charge density wave. The phase transition happens at $\frac{\tilde{V}_{c}}{2 t_{0}}=1$ which can be seen by mapping the model to the $X X Z$-Hamiltonian via a Jordan-Wigner transformation. In the $X X Z$-model the the phase transition happens at the isotropic Heisenberg point [22].

Another interesting limiting case is the adiabatic approximation that one recovers for a large mass of the molecules or $\omega_{0} \rightarrow 0$. A staggered displacement of the oscillators leads to the opening of a gap in the electronic spectrum and the formation of a charge density wave. This mechanism resembles the situation of a Peierls instability (cf. Sec. 2.2). In a mean field treatment, the charge density wave arises for all nonzero couplings $\gamma$ and the size of the gap is $[22,23]$ :

$$
\Delta=8 t_{0} \mathrm{e}^{-\frac{\pi t_{0} \omega_{0}}{\gamma^{2}}} .
$$

In Ref. [25] a two-cutoff renormalization group scheme was used to argue that for $\Delta<\omega_{0}$ nonadiabatic effects become relevant in the weak coupling regime. Therefore, the charge-density-wave order will only survive for $\Delta>\omega_{0}$.

\subsection{Phase diagram at half filling}

To investigate the Holstein model away from limiting cases, a variety of different techniques can be applied. For example, the momentum average approach 
introduced by Berciu and collaborators [32,34,100-103] is a specialized analytical method that has been successfully applied to the single electron problem in equilibrium. Quantum Monte Carlo techniques are maybe the most successful ansatz when dealing with electron-phonon coupled systems like the Holstein model $[22,24,27,31,34,35,104,105]$. They can produce very precise results for equilibrium properties.

In the realm of wave-function based methods, the Holstein model is a very challenging problem. The phonon number in the system is not conserved and, since they are bosons, their occupation on a single site can be very large. Using an occupation number basis results in huge local Hilbert spaces that pose a problem for techniques like exact diagonalization or the density matrix renormalization group. However, we have seen above that the ground state in the atomic limit is a product state which requires just a single state per site (cf. Eq. (3.12)). This result is a hint that the occupation number basis might not be the optimal one to describe eigenstates of the Holstein model numerically and that changing the local basis can improve the performance of wave-function based methods. In fact, numerical evidence suggests that in low temperature states as well as in real-time dynamics choosing a different local basis can reduce the necessary number of local states required for an accurate description [106-108]. Several different strategies have been developed to overcome the challenges posed by electron-phonon coupled systems to wave-function based methods [33] which will be discussed in Chs. 5 and 6 .

In Fig. 3.2, the phase diagram of the Holstein model at half filling is sketched in the $t_{0}-\gamma$-plane. From the small $t_{0}$ limit perturbation theory as well as the adiabatic approximation, a phase transition between a metallic Tomonaga-Luttinger liquid phase and an insulating charge-density-wave (CDW) phase can be deduced. For a fixed hopping matrix element $t_{0} / \omega_{0}$, the transition happens at a critical $\gamma / \omega_{0}$. The phase diagram for intermediate $t_{0} / \omega_{0}$ was computed by using DMRG and quantum Monte Carlo techniques [22-24, 104].

In the charge-density-wave phase, polarons order in a regular fashion. A gap opens to the excitation spectrum $[23,104]$ and the order parameter

$$
\mathcal{O}_{\mathrm{CDW}}=\frac{1}{N} \sum_{l=1}^{L}(-1)^{l}\left\langle n_{l}\right\rangle
$$

acquires a nonzero value [22], where $N$ is the number of electrons in the system, $L$ is the number of lattice sites and $\left\langle n_{l}\right\rangle$ is the electron density at site $l$. The nonzero value of $\mathcal{O}_{\mathrm{CDW}}$ leads to nonuniform displacements of the 


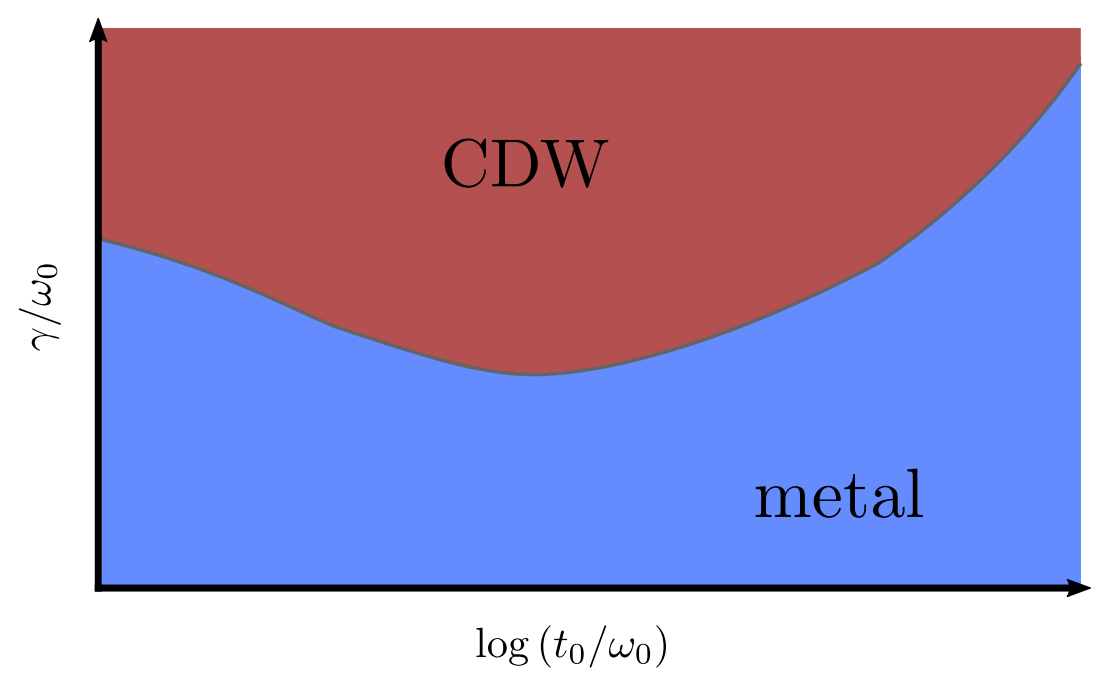

Figure 3.2: Phase diagram of the Holstein model at half filling sketched from the results in Refs. [23,24]. For all nonzero values of the hopping matrix element $t_{0}$ there is a transition from a metallic Tomonaga-Luttinger liquid phase to an insulating charge-density-wave phase upon increasing the coupling $\gamma$.

local harmonic oscillators such that the staggered displacement:

$$
\mathcal{O}_{\text {disp }}=\frac{1}{N} \sum_{l=1}^{L}(-1)^{l}\left\langle b_{l}^{\dagger}+b_{l}\right\rangle
$$

acquires a nonzero value as well. In fact, there is an exact relation between the expectation value of the electron density and the phonon displacement in all eigenstates of the Holstein Hamiltonian [22, 109]:

$$
2 \frac{\gamma}{\omega_{0}}\left\langle n_{l}\right\rangle=\left\langle b_{l}^{\dagger}+b_{l}\right\rangle
$$

which connects the electronic order parameter $\mathcal{O}_{\mathrm{CDW}}$ with the phononic order parameter $\mathcal{O}_{\text {disp }}$. 


\section{Chapter 4}

\section{Matrix-product-state methods}

In the following sections, numerical methods to calculate the time evolution of quantum states of lattice systems are introduced, as well as a technique to find the ground state of such systems. The modern formulations of these techniques are based on so called matrix product states (MPS) [110-112] and I will introduce these below. In the following derivations it is helpful to think of a collection of interacting spins or particles on a lattice as the system under consideration. This work is primarily focused on describing lattice models with electron-phonon coupling that pose special challenges to MPS techniques because of the large local Hilbert spaces. In Ch. 5 and Ch. 6 two extensions to the standard MPS techniques, introduced in this chapter, will be presented that are designed to overcome these challenges. The derivations in this chapter follow closely the review by Schollwöck Ref. [111].

\subsection{Matrix product states}

A general quantum state $|\psi\rangle$ on a lattice can be written in the following form:

$$
|\psi\rangle=\sum_{\left\{\sigma_{l}\right\}} c_{\sigma_{1} \ldots \sigma_{L}}\left|\sigma_{1} \ldots \sigma_{L}\right\rangle
$$

Here, $\left|\sigma_{1} \ldots \sigma_{L}\right\rangle=\left|\sigma_{1}\right\rangle \otimes \cdots \otimes\left|\sigma_{L}\right\rangle$, where $\left|\sigma_{l}\right\rangle$ is the state on a specific lattice site and $L$ is the total number of lattice sites. In the example of a spin- $1 / 2$ system the $\sigma_{l}$ would have the possible values $\uparrow$ or $\downarrow$. The sum runs over all possible combinations of local states. If every local Hilbert space $\mathcal{H}_{l}$ has a dimension $\operatorname{dim}\left(\mathcal{H}_{l}\right)=d$ then the dimension of the full Hilbert space $\mathcal{H}=\mathcal{H}_{1} \otimes \cdots \otimes \mathcal{H}_{L}$ is $\operatorname{dim}(\mathcal{H})=d^{L}$.

By regrouping the indices of the coefficients $c_{\sigma_{1} \ldots \sigma_{L}}$, one can bring them 
into a matrix form:

$$
|\psi\rangle=\sum_{\left\{\sigma_{l}\right\}} c_{\left[\sigma_{1}\right]\left[\sigma_{2} \ldots \sigma_{L}\right]}\left|\sigma_{1} \ldots \sigma_{L}\right\rangle
$$

such that $c$ can be read as a $d \times d^{L-1}$ matrix. A singular value decomposition (SVD) of this matrix can be performed:

$$
c=U S V^{\dagger}
$$

where $U$ and $V$ are (semi-)unitary matrices $\left(U^{\dagger} U=\mathbb{1}, V^{\dagger} V=\mathbb{1}\right)$ and $S$ is a diagonal matrix. The entries on the diagonal of the matrix $S$ are called singular values. $U$ and $S$ are both $d \times d$ matrices and $V^{\dagger}$ is a $d \times d^{L-1}$ matrix (Note that $V V^{\dagger} \neq \mathbb{1}$ ). With $A^{[1]}=U$ and $\tilde{c}=S V^{\dagger}$ the state can be written as:

$$
|\psi\rangle=\sum_{\left\{\sigma_{l}\right\}} \sum_{a_{1}} A_{\sigma_{1} a_{1}}^{[1]} \tilde{c}_{a_{1} \sigma_{2} \ldots \sigma_{L}}\left|\sigma_{1} \ldots \sigma_{L}\right\rangle
$$

One can now proceed in a similar fashion with $\tilde{c}$. One again regroups the indices such that it can be read as a matrix: $\tilde{c}_{a_{1} \sigma_{2} \ldots \sigma_{L}}=\tilde{c}_{\left[a_{1} \sigma_{2}\right]\left[\sigma_{3} \ldots \sigma_{L}\right]}$ and performs a SVD:

$$
\tilde{c}=\tilde{U} \tilde{S} \tilde{V}^{\dagger}
$$

With $A^{[2]}=\tilde{U}$ and $\hat{c}=\tilde{S} \tilde{V}^{\dagger}$ the state is in the form:

$$
|\psi\rangle=\sum_{\left\{\sigma_{l}\right\}} \sum_{a_{1} a_{2}} A_{\sigma_{1} a_{1}}^{[1]} A_{a_{1} \sigma_{2} a_{2}}^{[2]} \hat{c}_{a_{2} \sigma_{3} \ldots \sigma_{L}}\left|\sigma_{1} \ldots \sigma_{L}\right\rangle
$$

By performing overall $L-1$ SVDs one can bring the state into the form:

$$
|\psi\rangle=\sum_{\left\{\sigma_{l}\right\}} \sum_{a_{1} \ldots a_{L-1}} A_{\sigma_{1} a_{1}}^{[1]} A_{a_{1} \sigma_{2} a_{2}}^{[2]} \ldots A_{a_{L-1} \sigma_{L}}^{[L]}\left|\sigma_{1} \ldots \sigma_{L}\right\rangle .
$$

This form is called a matrix product state since one can get back every coefficient by calculating the matrix product $A_{\sigma_{1}}^{[1]} A_{\sigma_{2}}^{[2]} \ldots A_{\sigma_{L}}^{[L]}=c_{\sigma_{1} \ldots \sigma_{L}}$. The first matrix $A_{\sigma_{1}}^{[1]}$ is in fact a row vector and the last matrix $A_{\sigma_{L}}^{[L]}$ is a column vector such that the matrix product yields a scalar.

For a better readability, the labels $[l]$ of the matrices $A_{\sigma_{l}}^{[l]}$ will be omitted here. They can be distinguished by their indices. Furthermore, the indices $\sigma_{l}$ that label the physical state at the sites will be written as superscript indices. These are referred to as physical indices (their dimension is referred to as the physical dimension). The other indices $a_{l}$ are most commonly referred to 
as bond indices and their dimension is called the bond dimension. Finally, the summation over the bond indices will be omitted, too. From here on a summation over twice appearing indices is implied. Therefore, the state can be written more compact as:

$$
|\psi\rangle=\sum_{\left\{\sigma_{l}\right\}} A_{a_{1}}^{\sigma_{1}} A_{a_{1} a_{2}}^{\sigma_{2}} \ldots A_{a_{L-1}}^{\sigma_{L}}\left|\sigma_{1} \ldots \sigma_{L}\right\rangle
$$

A graphical representation of such a state will be helpful further down the road when equations and algorithms are explained. Such schematics have now become common in the MPS community [111,112]. Writing out all the summations over all the indices can be very confusing at times. Therefore, a shape is drawn for every tensor in an equation and for every index a line (these are called legs in the following) is attached to these shapes. If a leg connects two shapes the corresponding index is summed over. With these rules Eq. (4.8) results in Fig. 4.1.

To calculate the norm of the state $|\psi\rangle$ one has to evaluate:

$$
\begin{aligned}
\langle\psi \mid \psi\rangle= & \sum_{\left\{\sigma_{l}\right\},\left\{\sigma_{l}^{\prime}\right\}}\left\langle\sigma_{1} \ldots \sigma_{L}\right|\left(A^{\dagger}\right)_{a_{L-1}}^{\sigma_{L}} \ldots\left(A^{\dagger}\right)_{a_{2} a_{1}}^{\sigma_{2}}\left(A^{\dagger}\right)_{a_{1}}^{\sigma_{1}} \\
& \times A_{b_{1}}^{\sigma_{1}^{\prime}} A_{b_{1} b_{2}}^{\sigma_{2}^{\prime}} \ldots A_{b_{L-1}}^{\sigma_{L}^{\prime}}\left|\sigma_{1}^{\prime} \ldots \sigma_{L}^{\prime}\right\rangle \\
= & \left(A^{\dagger}\right)_{a_{L-1}}^{\sigma_{L}} \ldots\left(A^{\dagger}\right)_{a_{2} a_{1}}^{\sigma_{2}}\left(A^{\dagger}\right)_{a_{1}}^{\sigma_{1}} A_{b_{1}}^{\sigma_{1}} A_{b_{1} b_{2}}^{\sigma_{2}} \ldots A_{b_{L-1}}^{\sigma_{L}}
\end{aligned}
$$

A Graphical representation of Eq. (4.9) is shown in Fig. 4.2.

The properties of the SVD ensure that for the first $A$ matrix:

$$
\left(A^{\dagger}\right)_{a_{1}}^{\sigma_{1}} A_{b_{1}}^{\sigma_{1}}=\delta_{a_{1} b_{1}}
$$

and for the next $L-2$ matrices:

$$
\left(A^{\dagger}\right)_{a_{l} a_{l-1}}^{\sigma_{l}} A_{a_{l-1} b_{l}}^{\sigma_{l}}=\delta_{a_{l} b_{l}} .
$$

The evaluation of Eq. (4.9) then reduces to the product:

$$
\begin{gathered}
\langle\psi \mid \psi\rangle=\left(A^{\dagger}\right)_{a_{L-1}}^{\sigma_{L}} A_{a_{L-1}}^{\sigma_{L}} . \\
|\psi\rangle=\sum_{\left\{\sigma_{l}\right\}} A_{a_{1}} A a_{a_{2}}^{\sigma_{1}} \cdots a_{a_{L-1}}^{\sigma_{L}}\left|\sigma_{1} \ldots \sigma_{L}\right\rangle
\end{gathered}
$$

Figure 4.1: Graphical representation of a matrix product state. 


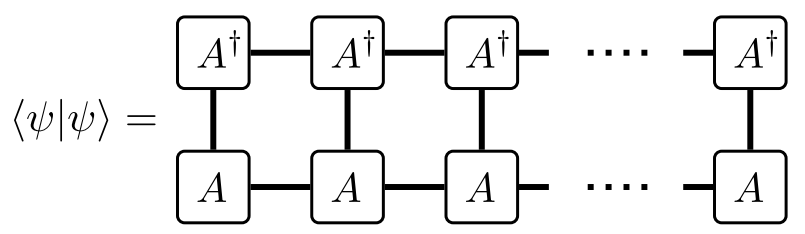

Figure 4.2: Graphical representation of the contractions needed to calculate the norm of a MPS.

The form Eq. (4.8) is therefore referred to as left-canonical or left-normalized. There is also a right-canonical or right-normalized form. It can be obtained by splitting off indices from $c_{\sigma_{1} \ldots \sigma_{L}}$ from the right and keeping the $V^{\dagger}$ matrices. The matrices in the right-canonical form have equivalent properties to the left-canonical form.

Last, one can also have a mixed-canonical form:

$$
|\psi\rangle=\sum_{\left\{\sigma_{l}\right\}} A_{a_{1}}^{\sigma_{1}} \ldots A_{a_{l-2} a_{l-1}}^{\sigma_{l-1}} M_{a_{l-1} a_{l}}^{\sigma_{l}} B_{a_{l} a_{l+1}}^{\sigma_{l+1}} \ldots B_{a_{L-1}}^{\sigma_{L}}\left|\sigma_{1} \ldots \sigma_{L}\right\rangle
$$

Here, all the left-canonical tensors are written as $A$ and all the right-canonical tensors as $B$. The tensor $M$ in the middle has neither of these properties and will be refer to as the center of the mixed-canonical form. Calculating the norm then reduces to:

$$
\langle\psi \mid \psi\rangle=\left(M^{\dagger}\right)_{a_{l} a_{l-1}}^{\sigma_{l}} M_{a_{l-1} a_{l}}^{\sigma_{l}}
$$

\subsubsection{Entanglement entropy}

So far, it is not clear why one would be interested in matrix product states. All that was done is rewriting the state in a more complicated way. The advantage of these states is that they provide a natural compression scheme and are especially suited to represent states with low entanglement entropy [111, 113-116].

Entanglement entropy is a measure of how strong different parts of a system are entangled with each other. To calculate the entanglement entropy of part of a system (part $A$ ) with the rest (part $B$ of the system, Fig. 4.3) one first calculates the density matrix of the whole system and then traces out one of the parts. By tracing out part $B$ one obtains the reduced density matrix of part $A$ :

$$
\rho_{A}=\operatorname{Tr}_{B}[|\psi\rangle\langle\psi|] .
$$


The (von Neumann) entanglement entropy of $A$ with $B$ is then defined as [115]:

$$
S_{\mathrm{vN}, A B}=\operatorname{Tr}\left[\rho_{A} \ln \rho_{A}\right] .
$$

To calculate the entanglement entropy one can use the Schmidt decomposition of the state [111]. Again, one divides the system in two parts $A$ and $B$ (Fig. 4.3) and writes the quantum state of the complete system in terms of full basis sets on $A$ and $B$ :

$$
|\psi\rangle=\sum_{\alpha, \beta} C_{\alpha \beta}|\alpha\rangle_{A}|\beta\rangle_{B} .
$$

After performing a SVD of the matrix $C$ one obtains:

$$
|\psi\rangle=\sum_{\alpha, \beta} U_{\alpha a} s_{a} \delta_{a b} V_{b \beta}^{\dagger}|\alpha\rangle_{A}|\beta\rangle_{B}
$$

Here, the diagonal matrix $S$ is written as its diagonal elements $s_{a}$ times a Kronecker delta. Since $U$ and $V^{\dagger}$ are unitary matrices their action on the basis states $|\alpha\rangle_{A}$ and $|\beta\rangle_{B}$ is that they produce new orthonormal basis sets of part $A$ and $B$ respectively. Therefore, the state can be written as:

$$
|\psi\rangle=\sum_{a, b} s_{a} \delta_{a b}|a\rangle_{A}|b\rangle_{B}=\sum_{a} s_{a}|a\rangle_{A}|a\rangle_{B} .
$$

The rightmost form is called the Schmidt decomposition of the state $|\psi\rangle$.

It is now straightforward to see that the reduced density matrix of $A$ can be written as:

$$
\rho_{A}=\sum_{a, b, c}\left\langle c\left|s_{a}\right| a\right\rangle_{A}|a\rangle_{B} s_{b}{ }_{A}\left\langle\left. b\right|_{B}\langle b \mid c\rangle_{B}=\sum_{a} s_{a}^{2} \mid a\right\rangle_{A A_{A}}\langle a| .
$$

This shows that the squares of the Schmidt values have to sum up to 1 for the state $|\psi\rangle$ to be normalized. Furthermore, it is now easy to calculate the entanglement entropy of $A$ with $B$ :

$$
S_{\mathrm{vN}, A B}=\operatorname{Tr}\left[\rho_{A} \ln \rho_{A}\right]=\sum_{a} s_{a}^{2} \ln s_{a}^{2} .
$$

\section{$A$}

\section{$B$}

Figure 4.3: A quantum system separated in two parts $A$ and $B$. 
Calculating the entanglement entropy of a matrix product state is also very straightforward. Consider the mixed-canonical form Eq. 4.13. To calculate the entanglement entropy between the first $l$ sites with the rest one first performs a SVD of the tensor $M$ where its indices are grouped as $\left[a_{l-1} \sigma_{l}\right]\left[a_{l}\right]$ :

$$
M_{a_{l-1} a_{l}}^{\sigma_{l}}=U_{a_{l-1} b}^{\sigma_{l}} s_{b} \delta_{b c}\left(V^{\dagger}\right)_{c a_{l}} .
$$

Because of the mixed-canonical form of the state $|\psi\rangle$ the diagonal elements $s_{b}$ are the Schmidt values of this state for the considered bipartition and the entanglement entropy can be calculated from them by using Eq. (4.21).

\subsubsection{Compressing a matrix product state}

Eq. (4.8) is just an exact rewriting of the coefficients. This also means that the matrix dimensions get larger (more precisely exponentially large) towards the middle of the system. However, the singular value decompositions guide one towards a compression of the state. Eq. (4.21) means that if the entanglement in the system is small, there are few big Schmidt values and the rest of them are much smaller. On the other hand, in a maximally entangled state all the Schmidt values are equally large. For states with low entanglement it is therefore sufficient to keep only the biggest Schmidt values without loosing much of the information about the state.

Consider the Schmidt decomposition Eq. (4.19) with a dimension $\zeta$ where all the Schmidt values are ordered such that $a_{1} \geq a_{2} \geq \cdots \geq a_{\zeta}$. The state is approximated by a compressed state $\left|\psi_{\text {comp }}\right\rangle$ where only the $\chi<\zeta$ biggest Schmidt values are kept:

$$
\left|\psi_{\text {comp }}\right\rangle=\sum_{a=1}^{\chi} s_{a}|a\rangle_{A}|a\rangle_{B} .
$$

The error that one makes is then:

$$
\||\psi\rangle-\left|\psi_{\text {comp }}\right\rangle \|^{2}=\sum_{a=\chi+1}^{\zeta} s_{a}^{2} .
$$

The sum of the squares of the discarded Schmidt values $\sum_{a=\chi+1}^{\zeta} s_{a}^{2}$ is referred to as the discarded weight. If one performs a sequence of SVDs to obtain a MPS it can be shown that the error is at most $\||\psi\rangle-\left|\psi_{\text {comp }}\right\rangle \|^{2} \leq 2 \epsilon_{l}(\chi)$ [113], where $\epsilon_{l}(\chi)$ is the discarded weight at site $l$ when truncating at a bond dimension $\chi$. 
These considerations show that MPSs can efficiently represent states with low entanglement. It can be shown, that ground states of short range Hamiltonians that have an energy gap to the higher excited states have a so called area law of entanglement $[114,115]$. This means that the entanglement entropy of a region of the system with the rest scales with the surface area of this region and not with its volume. For one-dimensional systems the surface of a connected region is at most two points such that the entanglement entropy in an area law state does not scale at all with the size of the region. In fact, the area law guarantees that the squares of the Schmidt values on the surface have a fast algebraic decay [114].

\subsubsection{Matrix product operators}

Analogous to quantum states, quantum operators can be represented as a collection of tensors as well. Consider a quantum operator $O$ that acts on the Hilbert space that the state $|\psi\rangle$, Eq. (4.1), lives in. Expanding the operator $O$ in the same basis one obtains:

$$
O=\sum_{\left\{\sigma_{l}\right\},\left\{\sigma_{l}^{\prime}\right\}} c_{\sigma_{1}^{\prime} \sigma_{1} \ldots \sigma_{L}^{\prime} \sigma_{L}}\left|\sigma_{1}^{\prime} \ldots \sigma_{L}^{\prime}\right\rangle\left\langle\sigma_{1} \ldots \sigma_{L}\right|
$$

Now, two indices that belong to the same site are split off at a time and SVDs are performed. This way one ends up with a matrix product operator (MPO):

$$
O=\sum_{\left\{\sigma_{l}\right\},\left\{\sigma_{l}^{\prime}\right\}} W_{a_{1}}^{\sigma_{1}^{\prime} \sigma_{1}} W_{a_{1} a_{2}}^{\sigma_{2}^{\prime} \sigma_{2}} \ldots W_{a_{L-1}}^{\sigma_{L}^{\prime} \sigma_{L}}\left|\sigma_{1}^{\prime} \ldots \sigma_{L}^{\prime}\right\rangle\left\langle\sigma_{1} \ldots \sigma_{L}\right|
$$

A graphical representation of the MPO is shown in Fig. 4.4(a) and its action on the state $|\psi\rangle$ in Fig. 4.4(b).

Putting the ingredients from above together one can formulate algorithms to perform simulations of quantum systems. These algorithms are quite straightforward to implement in a programming language because they just require the manipulation of tensors. In the following Secs. 4.2 and 4.3 methods to time evolve quantum states and to find the ground state of Hamiltonians will be introduced, respectively. Both algorithms are most efficient for one-dimensional systems.

\subsubsection{U(1)-symmetric matrix product states}

A lot of model Hamiltonians preserve a U(1)-symmetry like particle number conservation in Hubbard-type models, or conservation of total spin in 
Heisenberg-type models. It is common practice to use these symmetries in exact diagonalization techniques to reduce the numerical complexity [39]. By using basis states that belong to different symmetry sectors one can block diagonalize the Hamiltonian.

A similar strategy can be used for matrix product states $[55,56]$. Consider a state $|\psi\rangle_{N}$ in the subspace with quantum number $N$. If one further considers a bipartition of the system in part $A$ and $B$ similar to Eq. (4.17), one can choose basis states on the parts that are labeled by corresponding quantum numbers $N_{A}$ and $N_{B}$ :

$$
|\psi\rangle_{N}=\sum_{\alpha, \beta} C_{\alpha \beta}|\alpha\rangle_{N_{A}, A}|\beta\rangle_{N_{B}, B}
$$

A matrix element $C_{\alpha \beta}$ can only be non-zero if the corresponding quantum numbers on the parts sum up to the total quantum number: $N \stackrel{!}{=} N_{A}+N_{B}$. By ordering the basis states on the parts with respect to $N_{A}$ and $N_{B}$ one can block diagonalize the matrix $C_{\alpha \beta}$.

Now consider a tripartition of the state in a part $A$, a part $B$ and one site separating the two parts at site $l$. The state can be written as:

$$
|\psi\rangle_{N}=\sum_{\alpha, \sigma_{l}, \beta} C_{\alpha \sigma_{l} \beta}|\alpha\rangle_{N_{A}, A}\left|\sigma_{l}\right\rangle|\beta\rangle_{N_{B}, B}
$$

In this case the condition for the quantum numbers on the parts reads: $N \stackrel{!}{=}$ $N_{A}+\sigma_{l}+N_{B}$. From the values of $N_{A}$ and $\sigma_{l}$ the allowed basis states in part $B$ are predetermined.

(a)

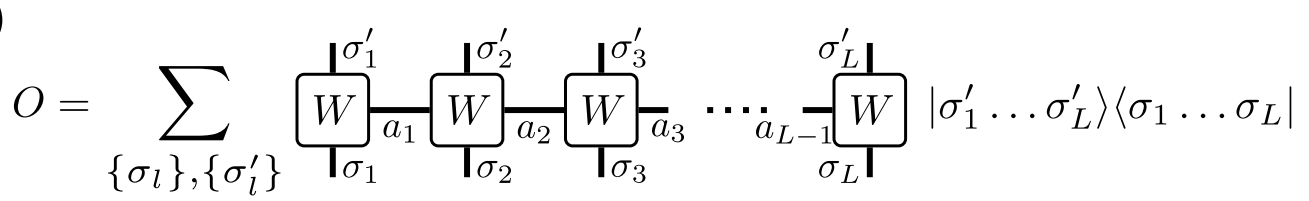

(b)

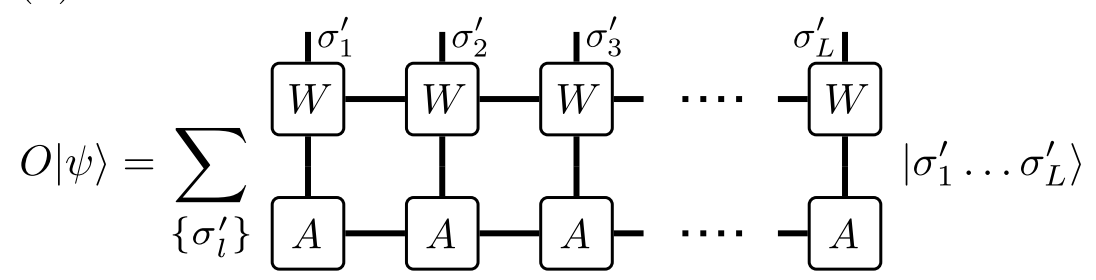

Figure 4.4: (a) Graphical representation of a matrix product operator. (b) Action of a MPO on a MPS. 
From these considerations one can see that the MPS and MPO tensors split into blocks with respect to the bond dimension and the local dimension. Manipulations of the tensors may be done blockwise which evidently reduces the numerical costs of MPS-based algorithms.

\subsection{Time evolution with matrix product states}

An algorithm for the time evolution of a quantum state that is particularly easy to understand and straightforward to implement was developed Vidal $[41,117]$. It is nowadays known as time evolving block decimation (TEBD). The algorithm, together with other closely related ones, is also referred to as time-dependent density-matrix renormalization group (tDMRG).

The starting point is yet another form of MPS, namely Vidal's $\Gamma \Lambda$-form [41]. It can be obtained by performing the same sequence of SVDs as is done to obtain Eq. (4.8). In the end, a unit matrix is inserted in between every pair of tensors. The unit matrix is decomposed into the diagonal matrices and their inverses that we obtained from the SVDs at the respective bonds:

$$
\mathbb{1}_{a_{l} a_{l}^{\prime}}=S_{a_{l} a_{l}^{\prime \prime}}\left(S^{-1}\right) a_{l}^{\prime \prime} a_{l}^{\prime} .
$$

By defining $\Lambda_{a_{l} a_{l}^{\prime}}=S_{a_{l} a_{l}^{\prime}}$ and $\Gamma_{a_{l-1}^{\prime} a_{l}}^{\sigma_{l}}=\left(S^{-1}\right)_{a_{l-1}^{\prime} a_{l-1}^{\prime \prime}} A_{a_{l-1}^{\prime \prime} a_{l}}^{\sigma_{l}}$ for all $l$ except $\Gamma_{a_{1}}^{\sigma_{1}}=A_{a_{1}}^{\sigma_{1}}$ the state has the Vidal or $\Gamma \Lambda$-form:

$$
|\psi\rangle=\sum_{\left\{\sigma_{l}\right\}} \Gamma_{a_{1}}^{\sigma_{1}} \Lambda_{a_{1} a_{1}^{\prime}} \Gamma_{a_{1}^{\prime} a_{2}}^{\sigma_{2}} \Lambda_{a_{2} a_{2}^{\prime}} \ldots \Lambda_{a_{L-1} a_{L-1}^{\prime}} \Gamma_{a_{L-1}^{\prime}}^{\sigma_{L}}\left|\sigma_{1} \ldots \sigma_{L}\right\rangle
$$

A schematic of the state in the $\Gamma \Lambda$-form is depicted in Fig. 4.5.

The neat property of this form is that one can readily calculate the entanglement entropy of every bisection of the state by just reading off the Schmidt values at the corresponding bond. Furthermore, one can get a mixed-canonical form that has its center at any site from the $\Gamma \Lambda$-form. By construction, all the tensors left of the center except the first one have the property that:

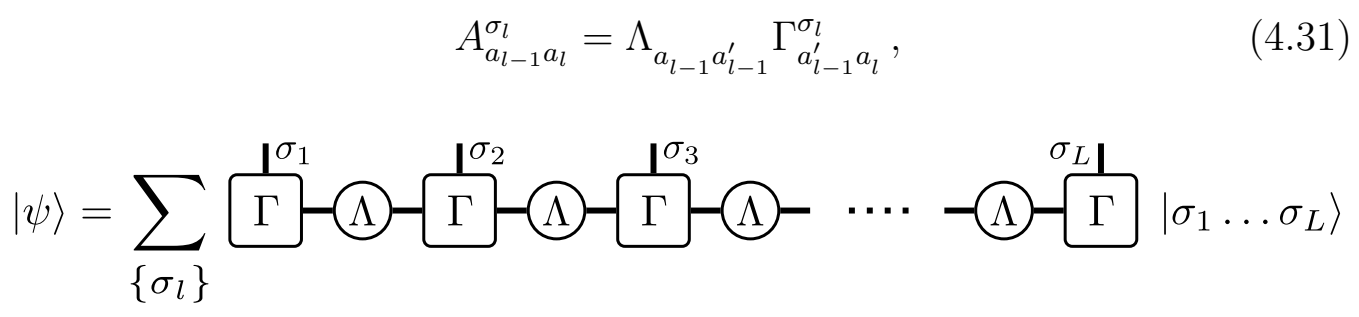

Figure 4.5: Graphical representation of a matrix product state in $\Gamma \Lambda$-form. 
where $A$ is a left-canonical tensor (the first tensor is already a left-canonical tensor). The center tensor can be calculated from the $\Gamma$ at that site and the two $\Lambda$ s left and right to it:

$$
M_{a_{l-1} a_{l}}^{\sigma_{l}}=\Lambda_{a_{l-1} a_{l-1}^{\prime}} \Gamma_{a_{l-1}^{\prime} a_{l}}^{\sigma_{l}} \Lambda_{a_{l} a_{l+1}^{\prime}} .
$$

From the SVDs we know that all the tensors to the right that remain now form a semi-unitary. This is however true for every bisection we can do. So by induction, starting at the second to last site as the center, one can show that:

$$
B_{a_{l-1} a_{l}}^{\sigma_{l}}=\Gamma_{a_{l-1} a_{l}^{\prime}}^{\sigma_{l}} \Lambda_{a_{l}^{\prime} a_{l}},
$$

where $B$ is a right-canonical tensor (with the exception of the last tensor where $\left.B_{a_{L-1}}^{\sigma_{L}}=\Gamma_{a_{L-1}}^{\sigma_{L}}\right)$.

The next step is to approximate the time-evolution operator by using a Trotter decomposition. Consider a (time independent) Hamiltonian with at most next nearest neighbour interaction in one dimension and with open boundary conditions. Then one can separate the Hamiltonian into two parts:

$$
H=H_{\text {odd }}+H_{\text {even }}=\sum_{l \text { odd }} h_{l}+\sum_{l \text { even }} h_{l},
$$

where $h_{l}$ is the part of the Hamiltonian that acts on site $l$ and $l+1$. Because of the prerequisites all the summands in $H_{\text {odd }}$ commute with each other, and so do all the summands in $H_{\text {even }}$.

The time-evolution operator that evolves a state by a small time interval $\delta t$ under the Hamiltonian $H$ is therefore:

$$
\begin{aligned}
e^{-\mathrm{i} H \delta t} & =e^{-\mathrm{i} H_{\text {odd }} \delta t / 2} e^{-\mathrm{i} H_{\text {even }} \delta t} e^{-\mathrm{i} H_{\text {odd }} \delta t / 2}+O\left((\delta t)^{3}\right) \\
& =\prod_{l \text { odd }} e^{-\mathrm{i} h_{l} \delta t / 2} \prod_{l \text { even }} e^{-\mathrm{i} h_{l} \delta t} \prod_{l \text { odd }} e^{-\mathrm{i} h_{l} \delta t / 2}+O\left((\delta t)^{3}\right) \\
& =\prod_{l \text { odd }} U_{l} \prod_{l \text { even }} U_{l} \prod_{l \text { odd }} U_{l}+O\left((\delta t)^{3}\right),
\end{aligned}
$$

where a second-order Trotter decomposition was used. The advantage of this decomposition is that the local operators $U_{l}$ only act on two neighboring sites. Fig. 4.6 shows a graphical representation of the tensor contractions needed to evolve a state of a five site system by a time step $\delta t$.

To apply a local time-evolution operator to a state one first has to calculate the two-site center tensor of a mixed canonical form:

$$
M_{a_{l-1} a_{l+1}^{\prime}}^{\sigma_{l} \sigma_{l+1}}=\Lambda_{a_{l-1} a_{l-1}^{\prime}} \Gamma_{a_{l-1}^{\prime} a_{l}}^{\sigma_{l}} \Lambda_{a_{l} a_{l}^{\prime}} \Gamma_{a_{l}^{\prime} a_{l+1}}^{\sigma_{l+1}} \Lambda_{a_{l+1} a_{l+1}^{\prime}} .
$$




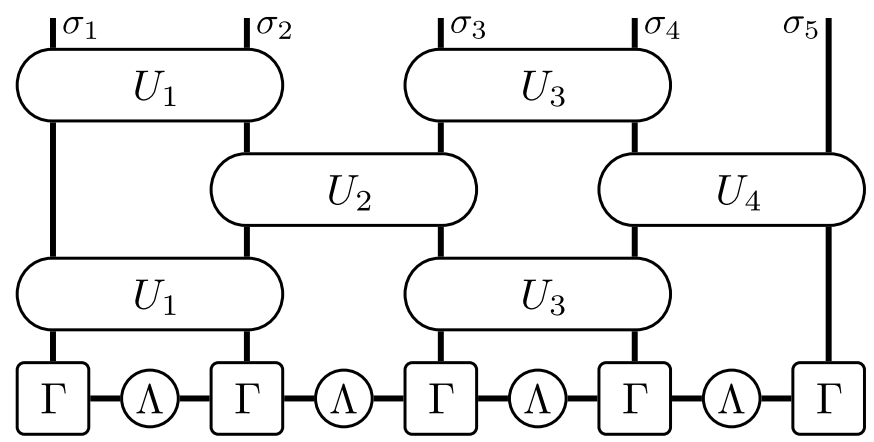

Figure 4.6: Graphical representation of the time evolution of a state of a five site system by a time step $\delta t$.

The mixed canonical form ensures that the reduced density matrix of the two sites can be calculated by just the tensor $M$ and changes to $M$ do not affect the rest of the state. Therefore one can now apply the local time-evolution operator $U_{l}$ to the tensor $M$ :

$$
\tilde{M}_{a_{l-1} a_{l+1}^{\prime}}^{\sigma_{l}^{\prime} \sigma_{l+1}^{\prime}}=\left(U_{l}\right)^{\sigma_{l}^{\prime} \sigma_{l+1}^{\prime} \sigma_{l} \sigma_{l+1}} M_{a_{l-1} a_{l+1}^{\prime}}^{\sigma_{l} \sigma_{l+1}} .
$$

To go back to the original $\Gamma \Lambda$-form one performs a SVD of the tensor $\tilde{M}$ :

$$
\tilde{M}_{a_{l-1} a_{l+1}^{\prime}}^{\sigma_{l}^{\prime} \sigma_{l+1}^{\prime}}=U_{a_{l-1} a_{l}}^{\sigma_{l}^{\prime}} S_{a_{l} a_{l}^{\prime}}\left(V^{\dagger}\right)_{a_{l}^{\prime} a_{l+1}^{\prime}}^{\sigma_{l+1}^{\prime}} .
$$

Then one can get the new $\tilde{\Gamma}_{\mathrm{s}}$ by contracting the inverse of the original $\Lambda$ s to $U$ and $V^{\dagger}$ from the left and right, respectively:

$$
\begin{aligned}
& \tilde{\Gamma}_{a_{l-1}^{\prime} a_{l}}^{\sigma_{l}^{\prime}}=\left(\Lambda^{-1}\right)_{a_{l-1}^{\prime} a_{l-1}} U_{a_{l-1} a_{l}}^{\sigma_{l}^{\prime}} \\
& \tilde{\Gamma}_{a_{l}^{\prime} a_{l+1}}^{\sigma_{l+1}^{\prime}}=\left(V^{\dagger}\right)_{a_{l}^{\prime} a_{l+1}^{\prime}}^{\sigma_{l+1}^{\prime}}\left(\Lambda^{-1}\right)_{a_{l+1}^{\prime} a_{l+1}},
\end{aligned}
$$

and identify $S$ by $\tilde{\Lambda}$.

In general, the bond-dimension between the sites $l$ and $l+1$ will grow after the application of the local time-evolution operator $U_{l}$. In order for it to not grow indefinitely and thus making all the calculations prohibitively expensive one truncates the bond dimension after the SVD. The error one makes is best controlled by setting a maximum truncation error and keeping as many singular values such that the squares of the singular values one does not keep is smaller than this maximum truncation error:

$$
\Delta_{\text {trunc }}<1-\sum_{a=1}^{r} s_{a}^{2}
$$


where $s_{r}$ is the smallest singular value one keeps. It is good practice to keep the truncation error smaller than the error from the Trotter decomposition. After the truncation the sum of the squares of the singular values should be normalized again to preserve the norm of the state.

\subsection{Ground-state search with matrix product states}

The next algorithm that will be discuss here is the density matrix renormalization group (DMRG) algorithm. It goes back to the groundbreaking work by White [40] (see also [42]). Later, the algorithm was reformulated in the language of matrix product states [111]. The algorithm is an iterative method to find the ground state of a given Hamiltonian and is again best suited for one-dimensional systems with short-range interactions.

To find the ground state one starts out by writing the state in the mixedcanonical form Eq. (4.13). In addition, one needs the Hamiltonian in MPO form:

$$
H=\sum_{\left\{\sigma_{l}\right\},\left\{\sigma_{l}^{\prime}\right\}} V_{a_{1}}^{\sigma_{1}^{\prime} \sigma_{1}} V_{a_{1} a_{2}}^{\sigma_{2}^{\prime} \sigma_{2}} \ldots V_{a_{L-1}}^{\sigma_{L}^{\prime} \sigma_{L}}\left|\sigma_{1}^{\prime} \ldots \sigma_{L}^{\prime}\right\rangle\left\langle\sigma_{1} \ldots \sigma_{L}\right|
$$

The energy expectation value of the state $|\psi\rangle$, assuming it is normalized, is (Fig. 4.7):

$$
\begin{aligned}
\langle\psi|H| \psi\rangle=\sum_{\left\{\sigma_{l}\right\},\left\{\sigma_{l}^{\prime}\right\}} & \left(B^{\dagger}\right)_{c_{L-1}}^{\sigma_{L}^{\prime}} \ldots\left(B^{\dagger}\right)_{c_{l+1} c_{l}}^{\sigma_{l+1}^{\prime}}\left(M^{\dagger}\right)_{c_{l} c_{l-1}}^{\sigma_{l}^{\prime}}\left(A^{\dagger}\right)_{c_{l-1} c_{l-2}}^{\sigma_{l-1}^{\prime}} \ldots\left(A^{\dagger}\right)_{c_{1}}^{\sigma_{1}^{\prime}} \\
& \times V_{b_{1}}^{\sigma_{1}^{\prime} \sigma_{1}} V_{b_{1} b_{2}}^{\sigma_{2}^{\prime} \sigma_{2}} \ldots V_{b_{L-1}}^{\sigma_{L}^{\prime} \sigma_{L}} \\
& \times A_{a_{1}}^{\sigma_{1}} \ldots A_{a_{l-2} a_{l-1}}^{\sigma_{l-1}} M_{a_{l-1} a_{l}}^{\sigma_{l}} B_{a_{l} a_{l+1}}^{\sigma_{l+1}} \ldots B_{a_{L-1}}^{\sigma_{L}} .
\end{aligned}
$$

The goal is to minimize the energy under the condition that the state is normalized. Therefore, one introduces a Lagrange multiplier $\lambda$ such that one

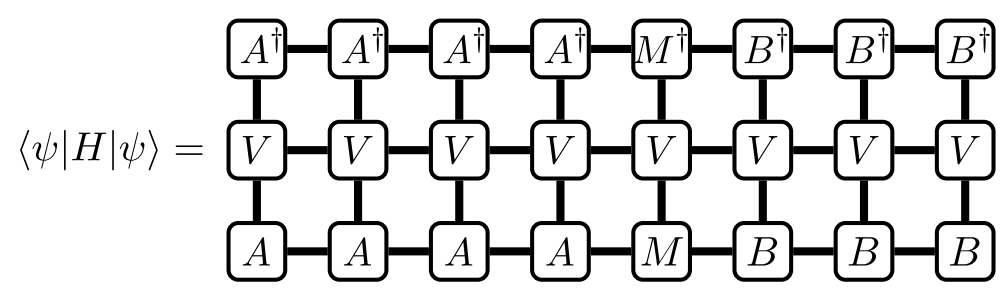

Figure 4.7: Graphical representation of the energy expectation value of a mixed-canonical state. 
has to extremize the equation:

$$
\langle\psi|H| \psi\rangle-\lambda\langle\psi \mid \psi\rangle=0
$$

with respect to the state $|\psi\rangle$. This is done iteratively by optimizing the tensor $M$ and then shifting the center of the mixed-canonical form to the next site to optimize the tensor on the next site.

Eq. (4.44) can be brought into the form of an eigenvalue equation where the tensor $M$ is the eigenvector and $\lambda$ is the eigenvalue. A graphical representation of the eigenvalue problem is shown in Fig. 4.8. The tensor $M^{\dagger}$ has been removed from both the expectation value of the energy and the norm. By contracting the left part of the expectation value of the energy to $L$ and the right part to $R$ one is left with:

$$
\begin{aligned}
L_{c_{l-1} b_{l-1} a_{l-1}} V_{b_{l-1} b_{l}}^{\sigma_{l}^{\prime} \sigma_{l}} R_{c_{l} b_{l} a_{l}} M_{a_{l-1} a_{l}}^{\sigma_{l}}=\lambda \delta_{c_{l-1} a_{l-1}} M_{a_{l-1} a_{l}}^{\sigma_{l}^{\prime}} \delta_{a_{l} c_{l}} \\
\Xi_{c_{l-1} a_{l-1} c_{l} a_{l}}^{\sigma_{l}^{\prime} \sigma_{l}} M_{a_{l-1} a_{l}}^{\sigma_{l}}=\lambda M_{c_{l-1} c_{l}}^{\sigma_{l}^{\prime}}
\end{aligned}
$$

where on the right side of the equation it was used that the tensors left of $M$ are left-normalized and the tensors right of $M$ are right-normalized. When reshaping the tensor $\Xi$ into a matrix with indices $\left[c_{l-1} \sigma_{l}^{\prime} c_{l}\right]\left[a_{l-1} \sigma_{l} a_{l}\right]$ and interpreting the tensor $M$ as a vector with indices $\left[a_{l-1} \sigma_{l} a_{l}\right]$ one can use a standard diagonalization technique to diagonalize $\Xi$. One is here only interested in the ground state so one can for example use the Lanczos algorithm [39]. The lowest eigenvalue that one obtains is the current estimate for the ground-state energy and the eigenvector is the improved tensor $M$.

If one sweeps from left to right through the system the updated tensor $M$ is then reshaped into a matrix with indices $\left[a_{l-1} \sigma_{l}\right]\left[a_{l}\right]$ and a SVD is performed. This way the center of the mixed-canonical form is moved to the next site to right. In a sweep from right to left the reshaping is done such that the tensor has indices $\left[a_{l-1}\right]\left[\sigma_{l} a_{l}\right]$.

\subsubsection{Two-site DMRG}

The algorithm introduced in the last section has two big problems. The energy of the state can only go down as one improves the local tensors while sweeping through the system which is good, but there is no guarantee that one ends up in the ground state. Instead, one could just reach a local minimum in the energy landscape. Furthermore, the bond dimension of the state can not grow during the algorithm. The dimension of $M$ before and after the update are fixed. Therefore, the entanglement in the state can only grow to a certain point determined by the bond dimensions in the initial state. 
One could try to artificially enlarge the tensor with zeros but there are more promising techniques.

A simple ansatz to let the bond dimension grow during the optimization is to optimize two site tensors at once. To do so one just has to build a mixed canonical form with a two-site center. During the optimization step one then has to solve the eigenvalue equation:

$$
\Xi_{c_{l-1} a_{l-1} c_{l+1} a_{l+1}}^{\sigma_{l}^{\prime} \sigma_{l+1}^{\prime} \sigma_{l} \sigma_{l+1}} M_{a_{l-1} a_{l+1}}^{\sigma_{l} \sigma_{l+1}}=\lambda M_{c_{l-1} c_{l+1}}^{\sigma_{l}^{\prime} \sigma_{l+1}^{\prime}}
$$

After the optimization one has to perform a SVD of the improved tensor $M$. The bond dimension between site $l$ and $l+1$ is then:

$$
\operatorname{dim}\left(\tilde{a}_{l}\right)=\min \left(\operatorname{dim}\left(\sigma_{l}\right) \operatorname{dim}\left(a_{l-1}\right), \operatorname{dim}\left(\sigma_{l+1}\right) \operatorname{dim}\left(a_{l+1}\right)\right) .
$$

Assuming that the dimensions of $a_{l-1}, a_{l}$ and $a_{l+1}$ before the update are of the same order, the bond dimension can grow by a factor of the local dimension $d=\operatorname{dim}\left(\sigma_{l}\right)$. This way, a bigger state space can be explored during the optimization and it is less likely that one gets stuck in a local minimum. To keep the numerical cost of the tensor manipulations at an acceptable level one has to truncate the bond dimension after the SVD. However, the numerical costs of the tensor manipulations that have to be done in the twosite DMRG algorithm presented here scale with a factor of $d^{2}$ worse than the scaling of the manipulations in the single-site algorithm presented in the previous section.
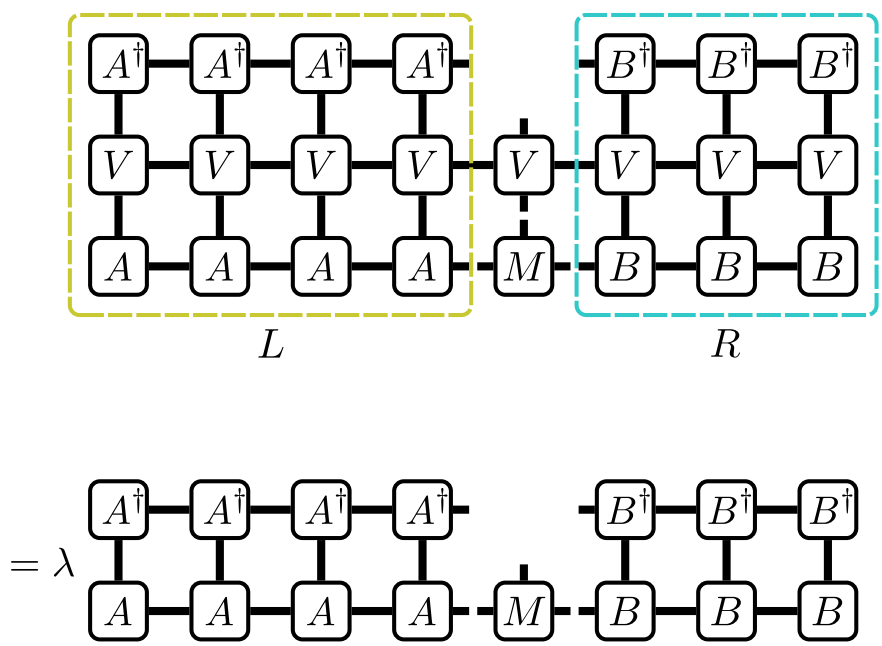

Figure 4.8: Graphical representation of the eigenvalue equation to be solved in a DMRG step. 
The drawback of a worse scaling with the local dimension is not too severe as long as only fermionic systems or spin systems are considered since the local dimension is small in these cases ( $d \leq 4$ in most cases, if $\mathrm{U}(1)$ symmetries can be utilized often a linear scaling with $d$ can be achieved). Problems can arise if bosonic degrees of freedom that do not obey a conservation law are present in a system. In this case the local dimension one has to consider can be substantial and it is desirable to use a single-site algorithm.

\subsubsection{Subspace expansion}

As mentioned earlier one can try to enlarge the MPS-tensors "by hand" to allow the bond dimension to grow and to avoid local minima in the energy landscape. The density-matrix perturbation developed by White [118] enlarges the bond dimension by introducing fluctuations that are suitable to allow for convergence. In modern MPS based algorithms a center-matrix formulation can be used to implement this strategy [55].

In the following, the so called subspace expansion developed by Hubig et al. [50] will be introduced. It is another strategy to enlarge the bond dimension and has a further improved scaling compared to the aforementioned methods.

The basic concept of the subspace expansion is simple. Consider a left to right sweep in a single-site DMRG calculation. After the optimization step on site $l$ one has a state in mixed-canonical form Eq. (4.13). Now the tensor $M$ on site $l$ and the tensor $B$ on site $l+1$ are enlarged such that only the bond dimension between those tensors grows. This way, when one does the optimization on site $l+1$, the new states that were added can be explored.

The expansion of $M$ is done by enlarging it with:

$$
\hat{M}_{c_{l-1}\left[b_{l} a_{l}\right]}^{\sigma_{l}^{\prime}}=\alpha L_{c_{l-1} b_{l-1} a_{l-1}} M_{a_{l-1} a_{l}}^{\sigma_{l}} V_{b_{l-1} b_{l}}^{\sigma_{l} \sigma_{l}^{\prime}}
$$

and by enlarging the tensor $B$ on site $l+1$ with zeros such that everything fits together. Up to here the state and therefore its energy is not changed since everything that was added is just multiplied by zeros. However, the bond dimension is enlarged quite substantially and, therefore, a truncation by SVD of the combined tensor $[M \hat{M}]$ is needed to keep the dimensionality at a manageable level. The notation $[M \hat{M}]$ means that the tensor $M$ is enlarged by $\hat{M}$ in such a way that the left bond dimension and the physical dimension do not change while the right bond dimension grows.

After this truncation, the energy of the state is usually larger than after the optimization on site $l$. This depends on the mixing factor $\alpha$. Therefore, the mixing factor has to be chosen such that it allows for enough new states to 
explore in the next optimization on site $i+1$ but does not hinder convergence altogether. During the sweeping, the mixing factor has to be adapted in an appropriate way. 


\section{Chapter 5}

\section{Local basis optimization}

The methods that I have described so far, namely TEBD and DMRG, are efficient methods to time evolve MPSs or find ground states of Hamiltonians, respectively. MPSs are used because they are able to efficiently represent states with limited entanglement. As explained in Sec. 4.1.2, in ground states of short-range Hamiltonians that have a gap to the excitation spectrum, the entanglement entropy follows an area law $[114,115]$. This results in the saturation of the bond dimension for a fixed precision as the system size is enlarged $[114,115]$.

In most practical applications, the scaling of MPS-based algorithms with the bond dimensions $\chi$ of the state is the limiting factor. In spin-1/2, spin- 1 , or fermionic models the local dimension is of less concern since $d \leq 4$. If $U(1)$ symmetries can be exploited for all degrees of freedom, the scaling is at most linear in the local dimension even in the Bose-Hubbard model (cf. Sec. 4.1.4). If, however, $U(1)$ symmetries can not be used, a large physical dimension slows down the algorithms, as two-site DMRG scales as $O\left(\chi^{3} d^{3}\right)$ and TEBD as $O\left(\chi^{3} d^{3}\right)$ or $O\left(\chi^{2} d^{4}\right)$.

The main subject of this thesis will be the study of electron-phonon coupled systems. In these systems, the number of phonons is not conserved and, because of the bosonic nature of the phonons, the local physical dimension is in principle infinite. If one uses an occupation-number basis for electrons and phonons, this immediately leads to problems. To even be able to write down the full Hamiltonian in matrix form, the Hilbert space has to have finite dimension, which is not true here.

One introduces an ad-hoc cutoff $M_{\mathrm{ph}}$ which is the maximal number of phonons per site. Then, the Hilbert space is finite and one can write down MPSs and MPOs in the usual way. Of course, one has to use the cut off $M_{\mathrm{ph}}$ as a variational parameter and increase it until the results of the calculations are independent of $M_{\mathrm{ph}}$. Depending on the problem at hand, this means 
increasing $d$ far beyond 10 and, therefore, calculations with wave-function based methods (like DMRG/TEBD) become very challenging.

However, different strategies have been developed to find more efficient basis sets than the naive occupation-number basis [33]. In the pseudo-site ansatz the phonon degrees of freedom on every site are mapped on a set of pseudo sites with two states each. This technique trades the large local Hilbert spaces for a longer chain with longer range hopping but smaller local Hilbert spaces which can be beneficial for DMRG [28,109]. The pseudo-site method will be discussed again in Ch. 7 where it will be used as a benchmark for other DMRG methods.

In the inhomogeneous modified variational Lang-Firsov transformation approach introduced by Weiße and Fehske [119], an effective Hamiltonian is derived that includes variational parameters. The variational parameters are then determined numerically [26].

A third method is the diagonalization in a limited functional space [30]. This ansatz generates a number of basis states by considering the action of parts of the Hamiltonian on a trial state. One obtains a set of states that are reachable by repeated action of the Hamiltonian on the state. The method exploits the fact that, at least in the Holstein model, the electronphonon couplings are local and the existence of polarons means that there is a spatial correlation between electrons and phonons. It was used in a number of studies of Holstein-type models [106, 120-127].

In this work, a very flexible method called local basis optimization (LBO) will be described and used. This method was introduced by Zhang, Jeckelmann, and White [29] and is very much inspired by DMRG . The method does not require any physical intuition about the system beforehand, however, I will discuss below under which circumstances the ansatz is especially beneficial.

As in Sec. 4.1.1, consider a bipartition of a system, yet, this time just one site is singled out. The single-site reduced density matrix at site $l$ is then:

$$
\rho_{l}=\operatorname{Tr}_{\substack{\sigma_{m} \\ m \neq l}}[|\psi\rangle\langle\psi|]
$$

By diagonalizing this single-site density matrix one gets a local basis transformation $P_{l}$ and the spectrum of the single-site density matrix in the diagonal matrix $D_{l}$ :

$$
\rho_{l}=P_{l} D_{l} P_{l}^{\dagger}
$$

If only a few of the eigenvalues of $\rho_{l}$ have considerable size and the rest of them is close to zero one can get away with only considering the eigenvectors 
of $\rho_{l}$ that correspond to the large eigenvalues and neglect the others. This yields a good approximation of the state $|\psi\rangle$ while the local physical dimension is reduced. The eigenvectors of the single-site reduced density matrix will be called optimal modes in the following and the number of modes that are kept are denoted by $d_{o}$ as opposed to $d$ for the full physical dimension.

Numerical data suggests that in electron-phonon problems the spectrum of the single-site reduced density matrix falls off exponentially at least in ground states $[106,107]$. The physical intuition behind this is that in these models polarons exist with a long lifetime. In this case, the diagonalization of the single-site reduced density matrix rotates the state into a polaron basis that is far more efficient in representing the state.

The advantage of this method is that the basis set that is chosen is locally always optimal. It is adapted to the state one considers and can be recalculated if the state changes.

In early efforts to use this ansatz optimal modes were calculated on small systems for a given parameter set. Then these optimal modes were used for calculations on larger systems [43-45]. In other methods, the optimal modes are calculated on a small subsystem of a larger system and the optimal modes from the subsystem are used for the rest of the sites [29,46], or the optimal modes are calculated just once in a warmup sweep [47]. Later, fully adaptive methods were developed that recalculate optimal modes whenever the state changes and for every lattice site individually [49, 51, 54, 108, 128, 129].

\subsection{TEBD with local basis optimization}

Now the local basis optimization is put to work and combined with the TEBD algorithm for the time evolution of quantum states [41,117] described in Sec. 4.2. The algorithm described here was used in Ref. [54] to study nonequilibrium phenomena in the half-filled Holstein model. It is a slightly modified version of the algorithm used by Brockt et al. [51-53] to study single electrons coupled to phonons. In the algorithm used by Brockt et al. the local time-evolution operators (4.35) are further approximated by a series expansion of the matrix exponential [53]. This way the sparseness of the Hamiltonian can be utilized in the application of the time-evolution operators to the state which yields a better scaling in the local dimension. In the algorithm outlined below the full matrix exponential is calculated and the local time-evolution operators are applied in the standard way.

Again, a MPS in $\Gamma \Lambda$-form is used where additionally every physical leg 
has a truncated local basis transformation $Q$ attached to it (Fig. 5.1):

$$
|\psi\rangle=\sum_{\left\{\sigma_{l}\right\}} Q^{\sigma_{1} \tilde{\sigma}_{1}} \Gamma_{a_{1}}^{\tilde{\sigma}_{1}} \Lambda_{a_{1} a_{1}^{\prime}} Q^{\sigma_{2} \tilde{\sigma}_{2}} \Gamma_{a_{1}^{\prime} a_{2}}^{\tilde{\sigma}_{2}} \Lambda_{a_{2} a_{2}^{\prime}} \ldots \Lambda_{a_{L-1} a_{L-1}^{\prime}} Q^{\sigma_{L} \tilde{\sigma}_{L}} \Gamma_{a_{L-1}^{\prime}}^{\tilde{\sigma}_{L}}\left|\sigma_{1} \ldots \sigma_{L}\right\rangle .
$$

The truncated local basis transformations $Q$ correspond to the respective $P_{l}$ from Eq. (5.2) where the $d-d_{o}$ columns of $P_{l}$ that correspond to the smallest eigenvalues in the spectrum of $\rho_{l}$ are dropped. The matrices $Q$ have dimension $d \times d_{o}$.

For the time evolution on the bond between sites $l$ and $l+1$ one forms the two-site center tensor as in Eq. (4.36). The local basis transformations $Q$ get contracted with the local time-evolution operator $U_{l}$ (Eq. (4.35)) from one site:

$$
\left(\tilde{U}_{l}\right)^{\sigma_{l}^{\prime} \sigma_{l+1}^{\prime} \tilde{\sigma}_{l} \tilde{\sigma}_{l+1}}=\left(U_{l}\right)^{\sigma_{l}^{\prime} \sigma_{l+1}^{\prime} \sigma_{l} \sigma_{l+1}} Q^{\sigma_{l} \tilde{\sigma}_{l}} Q^{\sigma_{l+1} \tilde{\sigma}_{l+1}} .
$$

At this point, it is important to contract the local basis transformations only from one site to the local time-evolution operator. The local basis might change during the time evolution and one has to make sure that the full local Hilbert spaces can be explored.

Nevertheless, the application of the transformed time-evolution operator $\tilde{U}_{l}$ to the two-site center tensor now scales with $\chi^{2} d_{o}^{2} d^{2}$ as opposed to $\chi^{2} d^{4}$ without the local basis optimization. After the application, one is left with the new two-site center tensor $\tilde{M}$ in the original basis:

$$
\tilde{M}_{a_{l-1} a_{l+1}^{\prime}}^{\sigma_{l}^{\prime} \sigma_{l+1}^{\prime}}=\left(\tilde{U}_{l}\right)^{\sigma_{l}^{\prime} \sigma_{l+1}^{\prime} \tilde{\sigma}_{l} \tilde{\sigma}_{l+1}} M_{a_{l-1} a_{l+1}^{\prime}}^{\tilde{\sigma}_{l} \tilde{\sigma}_{l+1}} .
$$

The next step is to transform this tensor into an optimized basis. For that purpose one has to calculate the single-site reduced density matrices on site $l$ and $l+1$ :

$$
\begin{aligned}
\left(\rho_{l}\right)^{\sigma_{l}^{\prime} \sigma_{l}^{\prime \prime}} & =\tilde{M}_{a_{l-1} a_{l+1}^{\prime}}^{\sigma_{l}^{\prime} \sigma_{\sigma^{\prime}}^{\prime}}\left(\tilde{M}^{\dagger}\right)_{a_{l+1}^{\prime} a_{l-1}^{\prime}}^{\sigma_{l+1}^{\prime} \sigma_{l}^{\prime \prime}} \\
\left(\rho_{l+1}\right)^{\sigma_{l+1}^{\prime} \sigma_{l+1}^{\prime \prime}} & =\tilde{M}_{a_{l-1} a_{l+1}^{\prime} \sigma_{l}^{\prime} \sigma_{l+1}^{\prime}}^{\sigma^{\prime}}\left(\tilde{M}^{\dagger}\right)_{a_{l+1}^{\prime} a_{l-1}^{\prime} \sigma_{l}^{\prime}}^{\sigma_{l+1}^{\prime}}
\end{aligned}
$$

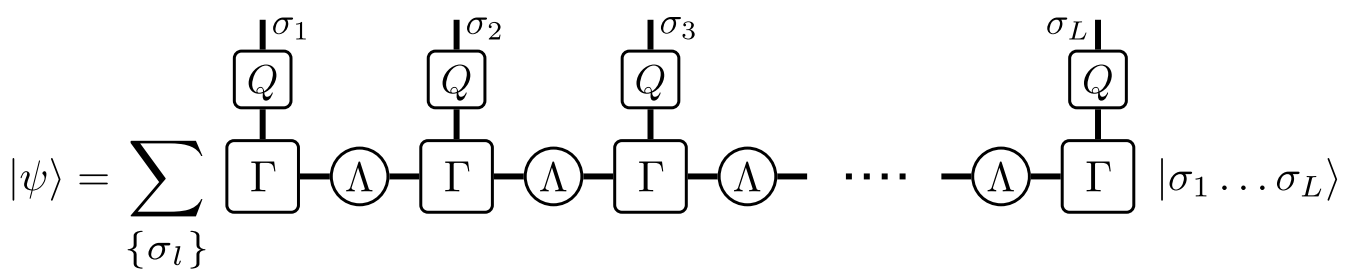

Figure 5.1: Graphical representation of a matrix product state in $\Gamma \Lambda$-form with additional local basis transformations. 
The diagonalization of $\rho_{l}$ and $\rho_{l+1}$ provides new optimal modes on the sites $l$ and $l+1$ and one can form new truncated local basis transformations $Q$ from them. By contracting the $Q^{\dagger}$ s with $\tilde{M}$, the bond tensor gets transformed into the new optimal basis. One can then continue with a SVD as in standard TEBD (Eqs. (4.38) - (4.40)) to go back to the $\Gamma \Lambda$-form. The SVD scales as $\chi^{3} d_{o}^{3}$ as opposed to $\chi^{3} d^{3}$ without the local basis optimization.

The numerical penalty is that one has to calculate the two single-site reduced density matrices which scales as $\chi^{2} d^{3}$. However, in most cases $d \ll \chi$ especially in the last time-evolution steps. Therefore, the numerical costs of the SVDs dominate most of the time and local basis optimization is actually beneficial.

A further benefit of this method is that all the observables can still be written in terms of the original basis that is most natural to work with. Whenever a measurement is necessary the corresponding MPOs can be contracted with the local basis transformations which is numerically cheap. The MPOs in the optimized basis can then be used to calculate expectation values.

In the implementation of the algorithm used in Ref. [54] and in this work instead of working with a fixed number of optimal modes a maximum discarded weight $\Delta_{\text {loc }}$ in the optimal modes is fixed. In the truncation step the $r$ optimal modes with the biggest eigenvalues are kept such that the sum of these eigenvalues $\sum_{i=1}^{r} d_{i}>1-\Delta_{\mathrm{loc}}$.

\subsection{Single-site ground-state DMRG with lo- cal basis optimization}

Now an algorithm will be introduced that combines the single-site groundstate DMRG with subspace expansion [50] introduced in Sec. 4.3.2 with local basis optimization. In later chapters the abbreviation DMRG3S+LBO will be used to refer to this algorithm. The method is heavily inspired by the one described in Ref. [49] but adjusted to work on electron-phonon systems. The algorithm was implemented by F. Dorfner and is described in detail in his doctoral thesis [48] and also in Refs. [38, 54].

Consider a matrix product state in mixed-canonical form that has a singlesite center. As in the previous section, the MPS has additional local basis 
optimization tensors $Q$ attached to every physical leg:

$$
\begin{aligned}
|\psi\rangle=\sum_{\left\{\sigma_{l}\right\}} Q^{\sigma_{1} \tilde{\sigma}_{1}} A_{a_{1}}^{\tilde{\sigma}_{1}} \ldots Q^{\sigma_{l-1} \tilde{\sigma}_{l-1}} A_{a_{l-2} a_{l-1}}^{\tilde{\sigma}_{l-1}} Q^{\sigma_{l} \tilde{\sigma}_{l}} M_{a_{l-1} a_{l}}^{\tilde{\sigma}_{l}} \\
\times Q^{\sigma_{l+1} \tilde{\sigma}_{l+1}} B_{a_{l} a_{l+1}}^{\tilde{\sigma}_{l+1}} \ldots Q^{\sigma_{L} \tilde{\sigma}_{L}} B_{a_{L-1}}^{\tilde{\sigma}_{L}}\left|\sigma_{1} \ldots \sigma_{L}\right\rangle .
\end{aligned}
$$

In the present form, the single-site reduced density matrix on site $l$ depends on the center tensor $M$ only:

$$
\left(\rho_{l}\right)^{\tilde{\sigma}_{l} \tilde{\sigma}_{l}^{\prime}}=M_{a_{l-1} a_{l}}^{\tilde{\sigma}_{l}}\left(M^{\dagger}\right)_{a_{l} a_{l-1}}^{\tilde{\sigma}_{l}^{\prime}} .
$$

The goal is to optimize the local basis with a DMRG like step. To do this the state is modified in such a way that the local reduced density matrix can be calculated from the (modified) $Q$ tensor only. This is done by SVD of the $M$ tensor where the physical index is one index and the two bond indices are interpreted as the second index of a matrix:

$$
M_{a_{l-1} a_{l}}^{\tilde{\sigma}_{l}}=Y^{\tilde{\sigma}_{l} \tau^{\prime}} \Lambda^{\tau^{\prime} \tau} X_{a_{l-1} a_{l}}^{\tau}
$$

Now, the tensors $Y$ and $\Lambda$ can be multiplied into the $Q$ tensor such that the single-site reduced density matrix can be calculated from the resulting tensor:

$$
\begin{aligned}
\tilde{Q}^{\sigma_{l} \tau} & =Q^{\sigma_{l} \tilde{\sigma}_{l}} Y^{\tilde{\sigma}_{l} \tau^{\prime}} \Lambda^{\tau^{\prime} \tau}, \\
\left(\rho_{l}\right)^{\sigma_{l} \sigma_{l}^{\prime}} & =\tilde{Q}^{\sigma_{l} \tau}\left(\tilde{Q}^{\dagger}\right)^{\tau \sigma_{l}^{\prime}}
\end{aligned}
$$

After these manipulations, an eigenvalue equation can be formulated similar to Eq. (4.46) where in this case, the $\tilde{Q}$ tensor is the eigenvector that one is searching for:

$$
\begin{aligned}
\Xi^{\iota \sigma_{l}^{\prime} \sigma_{l} \tau} & =L_{c_{l-1} b_{l-1} a_{l-1}}\left(X^{\dagger}\right)_{c_{l-1} c_{l}}^{\iota} V_{b_{l-1} b_{l}}^{\sigma_{l}^{\prime} \sigma_{l}} X_{a_{l-1} a_{l}}^{\tau} R_{c_{l} b_{l} a_{l}}, \\
\Xi^{\iota \sigma_{l}^{\prime} \sigma_{l} \tau} \tilde{Q}^{\sigma_{l} \tau} & =\lambda \tilde{Q}^{\sigma_{l}^{\prime} \iota}
\end{aligned}
$$

where the tensors $L$ and $R$ are defined as in Eq. (4.45). In further analogy to a standard DMRG optimization, the eigenvalue equation is solved for the the lowest eigenvalue and eigenvector with the Lanczos algorithm [39].

In the next step, the mixed-canonical form Eq. (5.8) is restored by SVD of the new optimized $\tilde{Q}$ tensor:

$$
\tilde{Q}^{\sigma_{l} \tau}=\tilde{Y}^{\sigma_{l} \tilde{\sigma}_{l}} \tilde{\Lambda}^{\tilde{\sigma}_{l} \tilde{\sigma}_{l}^{\prime}} \tilde{X}^{\tilde{\sigma}_{l}^{\prime} \tau} .
$$


One identifies $\tilde{Y}$ as the updated local basis transformation $Q$ at site $l$ and the new center tensor as:

$$
M_{a_{l-1} a_{l}}^{\tilde{\sigma}_{l}}=\tilde{\Lambda}^{\tilde{\sigma}_{l} \tilde{\sigma}_{l}^{\prime}} \tilde{X}^{\tilde{\sigma}_{l}^{\prime} \tau} X_{a_{l-1} a_{l}}^{\tau} .
$$

Here, a small subtlety arises since the number of optimal modes (the dimension of $\tilde{\sigma}_{l}$ ) can not grow during the optimization. The problem is similar to the one in conventional single-site DMRG Sec.4.3. This can be a problem, for example, if one chooses an initial state with a simple structure and only one optimal mode at every site. In further analogy to single-site DMRG, an enrichment step has to be implemented to ensure convergence.

After the optimization of the local basis transformation, a single-site DMRG step can be done to optimize the center tensor $M$ (cf. Sec.4.3). There is only one slight modification in Eq. (4.46), namely, that the MPO tensor $V$ is transformed to the new optimal basis:

$$
\tilde{V}_{b_{l-1} b_{l}}^{\tilde{\sigma}_{l}^{\prime} \tilde{\sigma}_{l}}=\left(Q^{\dagger}\right)^{\tilde{\sigma}_{l}^{\prime} \sigma_{l}^{\prime}} V_{b_{l-1} b_{l}}^{\sigma_{l}^{\prime} \sigma_{l}} Q^{\sigma_{l} \tilde{\sigma}_{l}} .
$$

The optimization of the local basis and the center tensor can be done in a loop until no further improvement can be detected. However, in the current implementation the optimization of both tensors is done just once or twice. After that one proceeds to the next site as explained in Sec. 4.3 .2 by doing a subspace expansion and truncation. 


\section{Chapter 6}

\section{Projected purification}

Very recently, an alternative ansatz to cope with large local dimensions in case of matrix-product-state methods was suggested, the so-called projected purification [37,130]. As discussed in Sec. 4.1.4 the numerical expenses of tensor manipulations in a MPS method can be reduced by exploiting $U(1)$ symmetries of Hamiltonians. The goal of the projected-purification ansatz is to artificially introduce such symmetries although the Hamiltonian does not have them in its original form.

Consider the Holstein Hamiltonian Eq. (3.4). It is evident that the number of fermions in the system is conserved by the Hamiltonian and, therefore, the MPS tensors on a certain site separate into two blocks with respect to the local dimension. However, the number of phonons in the system is not conserved since there are unpaired bosonic ladder operators in the interaction term of the Hamiltonian Eq. (3.3). With the aforementioned ad hoc phonon cutoff $M_{\mathrm{ph}}$ the effective local dimension of every tensor block is $M_{\mathrm{ph}}+1$ and it would be desirable to reduce this effective dimension even further.

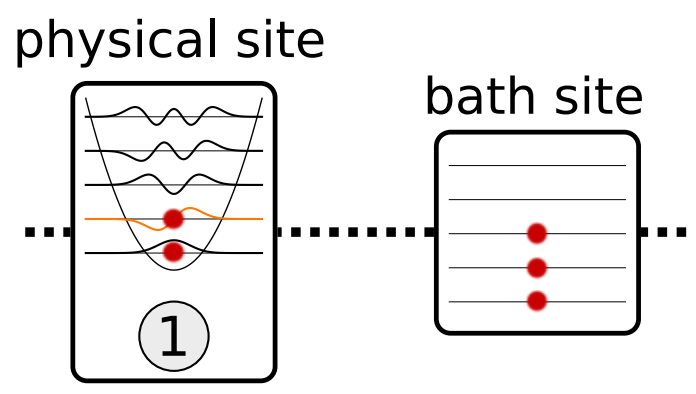

Figure 6.1: Projected purification ansatz: for every physical site a phonon bath site is introduced. The combined number of phonons on physical and associated bath site is conserved. 
Inspired by the purification method for finite-temperature matrix product states $[131,132]$ the projected purification ansatz introduces one additional bath site for every physical site of the system (Fig. 6.1). The bath sites serve as phonon reservoirs such that a phonon that is created on a physical site hops to it from the corresponding bath site. Similarly, the phonon hops back to the bath site if it is annihilated on the physical site. The sum of phonons on the physical and bath site is conserved and, therefore, an artificial $U(1)$ symmetry is introduced.

Formally, the introduction of the bath sites maps the problem to an enlarged Hilbert space with basis states of the form:

$$
\cdots \otimes\left|n_{l}, n_{\mathrm{ph}, l}\right\rangle_{\mathrm{p}} \otimes\left|n_{\mathrm{b}, l}\right\rangle_{\mathrm{b}} \otimes \ldots
$$

where $n_{l}$ is the electron number on site $l, n_{\mathrm{ph}, l}$ the phonon number, and $n_{\mathrm{b}, l}$ the phonon number on the corresponding bath site. By enforcing the phonon number conservation on every pair of physical and bath site, one restricts the states to a subspace of the enlarged Hilbert space:

$$
n_{\mathrm{ph}, l}+n_{\mathrm{b}, l} \stackrel{!}{=} M_{\mathrm{ph}} \quad \forall l .
$$

Within this subspace, every state can be uniquely identified with a state from the original Hilbert space:

$$
\cdots \otimes\left|n_{l}, n_{\mathrm{ph}, l}\right\rangle_{\mathrm{p}} \otimes\left|M_{\mathrm{ph}}-n_{\mathrm{ph}, l}\right\rangle_{\mathrm{b}} \otimes \ldots \quad \longrightarrow \quad \cdots \otimes\left|n_{l}, n_{\mathrm{ph}, l}\right\rangle \otimes \ldots
$$

To achieve the mapping, balancing operators that only act on the bath sites have to be introduced. These balancing operators are defined by their action on the bath sites:

$$
\begin{aligned}
& { }_{\mathrm{b}}\left\langle n_{\mathrm{b}, l}^{\prime}\left|\beta_{\mathrm{b}, l}^{\dagger}\right| n_{\mathrm{b}, l}\right\rangle_{\mathrm{b}}=\delta_{n_{\mathrm{b}, l}^{\prime}, n_{\mathrm{b}, l}+1}, \\
& { }_{\mathrm{b}}\left\langle n_{\mathrm{b}, l}^{\prime}\left|\beta_{\mathrm{b}, l}\right| n_{\mathrm{b}, l}\right\rangle_{\mathrm{b}}=\delta_{n_{\mathrm{b}, l}^{\prime}, n_{\mathrm{b}, l}-1},
\end{aligned}
$$

and commute with all operators on the physical sites:

$$
\begin{aligned}
& {\left[\beta_{\mathrm{b}, l}^{(\dagger)}, b_{\mathrm{p}, m}^{(\dagger)}\right]=0,} \\
& {\left[\beta_{\mathrm{b}, l}^{(\dagger)}, c_{\mathrm{p}, m}^{(\dagger)}\right]=0,}
\end{aligned}
$$

where $\beta_{\mathrm{b}, l}^{\dagger} / \beta_{\mathrm{b}, l}$ are the balancing operators acting on the bath sites, $b_{\mathrm{p}, l} / b_{\mathrm{p}, l}^{\dagger}$ are the phonon operators acting on the physical sites, and $c_{\mathrm{p}, l}^{\dagger} / c_{\mathrm{p}, l}$ are the fermionic operators acting on the physical sites. 
The Hamiltonian in the enlarged Hilbert space is then constructed by replacing the ladder operators acting on the original Hilbert space as follows:

$$
\begin{aligned}
& b_{l} \longrightarrow b_{\mathrm{p}, l} \beta_{\mathrm{b}, l}^{\dagger}, \\
& b_{l}^{\dagger} \longrightarrow b_{\mathrm{p}, l}^{\dagger} \beta_{\mathrm{b}, l}, \\
& c_{l}^{(\dagger)} \longrightarrow c_{\mathrm{p}, l}^{(\dagger)} .
\end{aligned}
$$

It is important to note that the balancing operators are chosen such that the matrix elements of the operators in the original Hilbert space and the corresponding matrix elements of the operators in the enlarged Hilbert space are identical. Therefore, balancing operators do not fulfil standard bosonic commutation relations.

The introduction of the balancing operators combined with the unique identification of states between original and enlarged Hilbert space allows one to completely work in the enlarged space. For example, the Holstein Hamiltonian Eq. (3.4) takes the form:

$$
\begin{aligned}
H_{\mathrm{Hol}, \mathrm{p}, \mathrm{b}}= & -t_{0} \sum_{l=1}^{L}\left(c_{\mathrm{p}, l}^{\dagger} c_{\mathrm{p}, l+1}+c_{\mathrm{p}, l+1}^{\dagger} c_{\mathrm{p}, l}\right)+\omega_{0} \sum_{l=1}^{L} b_{\mathrm{p}, l}^{\dagger} b_{\mathrm{p}, l} \\
& -\gamma \sum_{l=1}^{L} n_{l}\left(b_{\mathrm{p}, l}^{\dagger} \beta_{\mathrm{b}, l}+b_{\mathrm{p}, l} \beta_{\mathrm{b}, l}^{\dagger}\right),
\end{aligned}
$$

and similarly, other operators can be constructed with the replacement rules (6.8), (6.9), and (6.10).

By introducing artificial phonon number conservations on every pair of physical and bath sites the projected-purification ansatz reduces the effective local dimension of every tensor block in the MPS to one. Therefore, matrix manipulations can be done way more efficiently than without the artificial symmetries. For example, it becomes feasible to use a standard two-site DMRG algorithm to calculate ground states even with very large local dimensions [37].

Integrating this method into an existing code, that is able to utilize $U(1)$ symmetries, is straight forward. One just has to include the additional bath sites and needs to define the balancing operators, Eqs. (6.4) and (6.5), correctly.

The drawback of the algorithm is clearly the doubling of the system size due to the introduction of the bath sites. This also means that nearest neighbor interactions between physical sites become next nearest neighbor interactions. Furthermore, the information that is stored in the local basis transformations in case of the LBO method has to be stored in the MPS 
tensors here, as well. As we will see in Sec. 7.3 this enlarges the bond dimension needed compared to an MPS with LBO. A detailed analysis of this phenomenon with an estimate of the increase in bond dimension is given in Refs. [37,130]. 


\section{Chapter 7}

\section{Ground-state calculations in the Holstein model}

In this chapter, the ground-state DMRG3S+LBO method introduced in Sec. 5.2 will be put to work to study the half-filled Holstein model. As explained above the algorithm is especially tailored to tackle electron-phonon coupled systems. The results that I will show should demonstrate the accuracy and capabilities of the method. Furthermore, I will discuss challenges and pitfalls that one could encounter when working with this ansatz. The content of this chapter is published in Ref. [38].

I will compare my results with results obtained by a projected-purificton DMRG (PP-DMRG) method (Sec. 6) and with results obtained with a pseudosite DMRG (PS-DMRG) method. In the PP-DMRG method, a standard two site DMRG solver was used. The maximal number of phonons per site is set to $M_{\mathrm{ph}}=63$ for all data shown in the following. This is done since tensor blocks that have a vanishing occupation can be erased completely in the used implementation and thus a local dimension that is too large does not slow down the algorithm noticeably. The PP-DMRG data shown below was provided by Sebastian Paeckel from the Ludwig-Maximilians-Universität München and Thomas Köhler from the Uppsala University [133].

The PS-DMRG method was developed by Jeckelmann and White [28] (see also Ref. [33]). In this ansatz the $M_{\mathrm{ph}}+1$ phonon levels on every site are mapped to a number of additional (pseudo) sites that have two states each. The occupations of these pseudo sites represent the phonon level occupations as binary numbers (Fig. 7.1). Therefore, the ansatz cures the problem of large local Hilbert spaces in electron-phonon coupled systems. The penalty is that the system size is enlarged by $L$ times the number of pseudo sites and that the coupling terms become quite complicated as well as long range. Furthermore, the bond dimension that one needs to account 


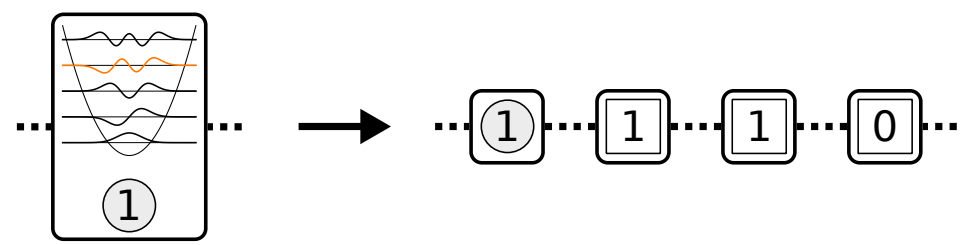

Figure 7.1: Pseudo-site ansatz: the phonon degrees of freedom are mapped to a number of (pseudo) sites with two states each that represent the phonon state as a binary number.

for a certain precision is enlarged for this method compared to the LBO ansatz. Similar to the projected purification, the information that is stored in the local basis transformations in case of the LBO has to be encoded in the bond dimension in case of the pseudo-site method. The PS-DMRG produces reliable results and was successfully used to study ground states in the Holstein and Hubbard-Holstein model (see e.g. Refs. [28, 109, 134, 135]). The PP-DMRG data shown below was provided by Eric Jeckelmann from the Leibniz Universität Hannover [136].

\subsection{Properties of the DMRG3S+LBO imple- mentation}

The implementation of the DMRG3S+LBO algorithm used here [48] utilizes the fermion number conservation of the Holstein Hamiltonian Eq. (3.4). This means that the single site reduced density matrix Eq. (5.1) separates into two symmetry blocks (cf. Sec. 4.1.4), one block for no fermion at the site and one block for one fermion at the site. Optimal modes can be calculated for the two blocks separately and in the DMRG calculations, the maximum number of optimal modes $d_{\mathrm{o}}$ per block is fixed. In the truncation procedure, the weights corresponding to the optimal modes from both blocks are combined in one ordered list. The two blocks of the local basis transformation matrix are then filled with the optimal modes according to the size of the corresponding weights until one of the blocks reaches the maximum size of $d_{\mathrm{o}}$ optimal modes. The other block is then filled with zeros until it reaches the same size as the first block. This is done to allow the algorithm to explore the full number of $d_{\mathrm{o}}$ optimal modes in both symmetry sectors the next time the optimal modes at the site are adapted (cf. Sec. 5.2).

The truncation in the bond dimension of the matrix product states is done in a simpler way. Again the singular values from the many symmetry sectors (cf. Eq. (4.27)) are combined to one list and the states corresponding 
to the largest singular values are kept until the maximum bond dimension is reached. Here, a small technical difficulty arises since it might happen that whole symmetry blocks vanish which can spoil the consistency with the tensors on neighboring sites. Therefore, the symmetry blocks that vanish are kept with a single state and the weight of the block is set to zero. The bond dimension $\chi$ that I will refer to in the following is the number of states kept with non-zero weight.

Additionally to the maximum bond dimension, a maximum truncation error $\Delta_{\text {trunc }}$ is specified and the algorithm will add states until either the maximum specified bond dimension is reached or the sum of the singular values squared on the bond reaches $1-\Delta_{\text {trunc }}$. The maximum truncation error is set to $\Delta_{\text {trunc }}=10^{-14}$, and, therefore, close to machine precision, in the following calculations.

As already mentioned in Sec. 4.3.2, an appropriate adaption of the mixing factor $\alpha$ in the subspace expansion is key to a fast convergence of the algorithm that avoids local energy minima. It turns out that a large mixing factor $\alpha \sim 1$ or even larger is beneficial in the first few sweeps of the simulation to explore large regions of the Hilbert space. In later sweeps, the mixing factor should be reduced to allow the algorithm so settle into a specific state. As suggested in Ref. [50], the mixing factor is adapted after the truncation step is finished and the center tensor of the mixed canonical form is shifted to the next site (cf. Sec. 4.3.2). One compares three energies: the energy after the truncation of the previous site $E_{\text {last }}$, the energy after the DMRG optimization and before the subspace expansion is done $E_{\mathrm{opt}}$, and the energy after the subspace expansion and truncation is done $E_{\text {trunc }}$. From the comparison of these three energies, an adaption factor $\eta$ for $\alpha$ is chosen as follows:

$$
\begin{array}{ll}
\Delta_{\text {opt }}:=\frac{E_{\text {last }}-E_{\mathrm{opt}}}{\left|E_{\mathrm{opt}}\right|}, \quad \Delta_{\text {trunc }}:=\frac{E_{\text {trunc }}-E_{\mathrm{opt}}}{\left|E_{\mathrm{opt}}\right|} & \\
\text { if }\left|\Delta_{\text {opt }}\right|<10^{-14} \text { or }\left|\Delta_{\text {trunc }}\right|<10^{-14}: & \eta=1 \\
\text { else if } \quad \Delta_{\text {trunc }}<0: & \eta=2\left(1+\frac{\left|\Delta_{\text {trunc }}\right|}{\left|\Delta_{\text {opt }}\right|}\right) \\
\text { else if } \quad \frac{\left|\Delta_{\text {trunc }}\right|}{\left|\Delta_{\text {ott }}\right|}<0.05: & \eta=1.2-\frac{\left|\Delta_{\text {trunc }}\right|}{\left|\Delta_{\text {opt }}\right|} \\
\text { else if } \quad \frac{\left|\Delta_{\text {trunc }}\right|}{\left|\Delta_{\text {opt }}\right|}>0.3: & \eta=\frac{1}{1.01} \\
\text { else }: & \eta=1
\end{array}
$$

The adaption factor $\eta$ is then further restricted to be no smaller than 0.99 and no larger than 1.01. The new mixing factor is then $\alpha^{\prime}=\eta \alpha$. However, the mixing factor is always kept between $10^{-9}$ and 100 . Furthermore, no subspace expansion is done if the mixing factor falls below $10^{-8}$. This 
adaption strategy has proven to be successful in producing the results below. The exact numbers in the adaption strategy are, however, results of practical experience and trial-and-error. Therefore, if might be necessary to change these numbers if other systems than the Holstein model are considered.

Furthermore, the chosen initial state for the ground-state search has a huge impact on the convergence pace. One can speed up the convergence by using an initial state that already has key features of the ground state built into it. Of course, there is also a danger in this strategy since one biases the algorithm towards a certain kind of state that might not sit in the global energy minimum. A common strategy in DMRG simulations is to use a procedure similar to infinite-system DMRG [40,42] where sites are added in the middle of the chain until the desired chain length is reached. This approach produces an initial state that is already a very good guess for the ground state.

To produce the ground-state data for the Holstein model at half filling that I will show below, a different strategy was chosen. In a preparation run, the coupling to the phonons is set to zero $(\gamma=0)$ and a ground state with a rather small bond dimension $\chi$ is produced. Then, the ground state for $\gamma=0$ is used as the initial guess state for the next run where the coupling is set to a small finite value. The bond dimension for this run is kept at a similar small value.

This procedure of gradually increasing the coupling $\gamma$ from run to run and always taking the ground state from the last run as an initial guess for the next is repeated until the desired coupling strength is reached. Then, the maximal bond dimension $\chi$ is gradually increased from run to run until the desired precision is reached. This procedure was found to be successful in preventing the algorithm from getting stuck in local energy minima and producing reliable results.

An example for this strategy at $L=51, N=25, \omega_{0} / t_{0}=1$ and $\gamma / t_{0}=2$ is shown in Fig. 7.2. The maximal bond dimension $\chi$ is set to 20 , the maximal number of phonons per site $M_{\mathrm{ph}}$ is set to 31 and $d_{\mathrm{o}}=5$ optimal modes are allowed.

Three datasets are shown. In the first run $\left(\mathcal{R}_{1}\right.$, red dots $)$, the initial guess state is the ground state at $\omega_{0} / t_{0}=1, \gamma / t_{0}=1.5$, and $\chi=20$ and the mixing factor $\alpha$ is fixed to 1 for the first 30 sweeps $\left(s_{\alpha=1}=30\right)$. In the second run $\left(\mathcal{R}_{2}\right.$, blue stars), the initial guess state is the same as in $\mathcal{R}_{1}$ but the mixing factor $\alpha$ is fixed to 1 only in the first run $\left(s_{\alpha=1}=1\right)$. In the third run ( $\mathcal{R}_{3}$, green diamonds), the initial guess state is the ground state at $\gamma / t_{0}=0$ and $\chi=15$ and the mixing factor $\alpha$ is fixed to 1 for the first 30 sweeps $\left(s_{\alpha=1}=30\right)$ as in $\mathcal{R}_{1}$.

The inset of Fig. 7.2(a) shows the density distribution in the initial guess 


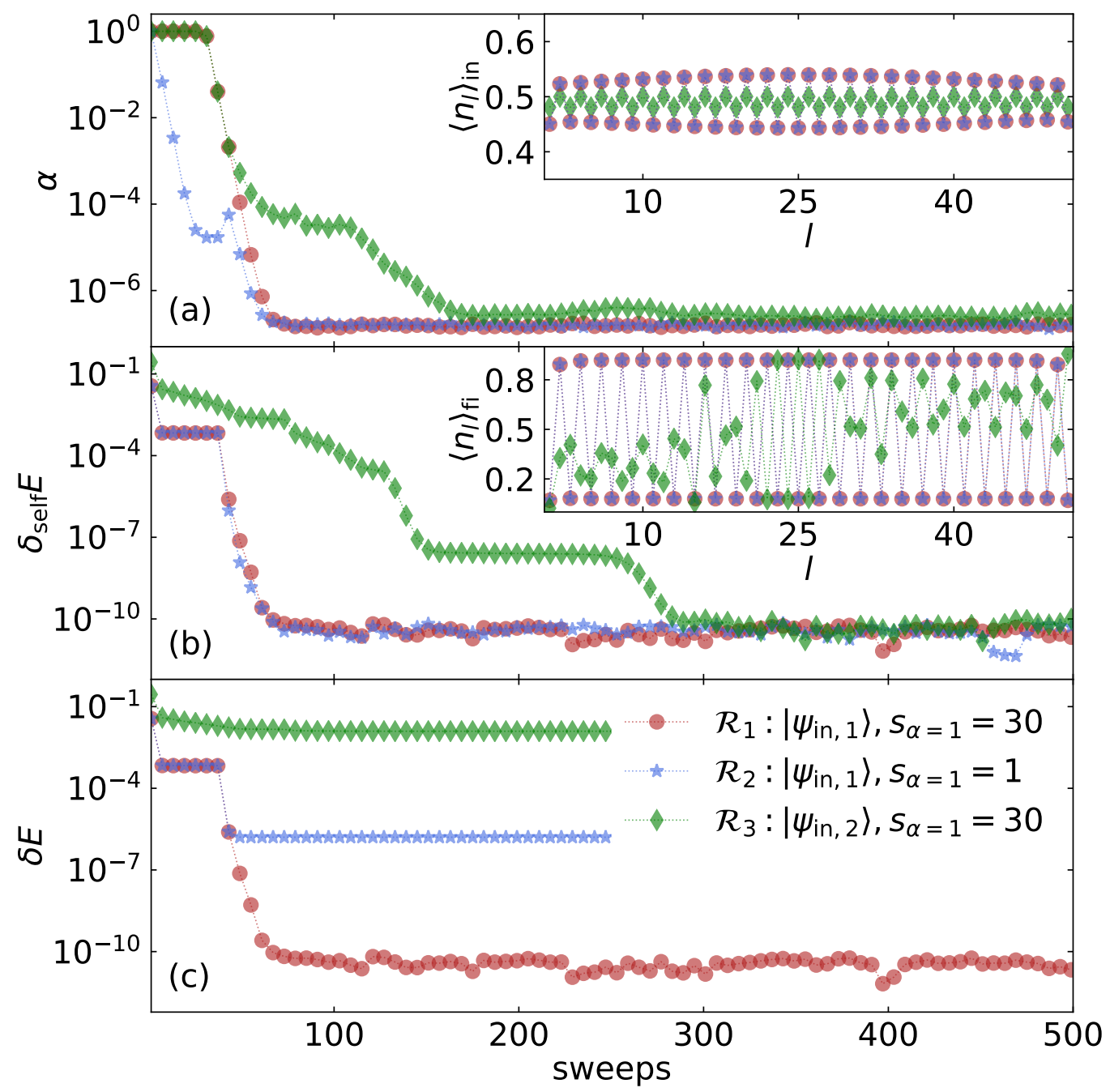

Figure 7.2: (a) Evolution of the subspace-expansion mixing factor $\alpha$ during the sweeping. (b) Evolution of the energy during the sweeping with respect to the best energy estimate in the runs. (c) Evolution of the energy during the sweeping with respect to the best energy estimate in run with initial state $\left|\psi_{\text {in }, 1}\right\rangle$ and $s_{\alpha=1}=30$. Inset of (a): electron density in the initial states. Inset of (b): electron density in the final states. System parameters are $L=51$, $N=25, \omega_{0} / t_{0}=1$ and $\gamma / t_{0}=2$. In all runs $\chi=20, M_{\mathrm{ph}}=31$ and $d_{\mathrm{o}}=5$.

states. It is evident that the ground state at $\omega_{0} / t_{0}=1$ and $\gamma / t_{0}=1.5($ $\left|\psi_{\text {in, } 1}\right\rangle: \mathcal{R}_{1}$ and $\mathcal{R}_{2}$ ) has more structure in the density profile than the ground state at $\gamma / t_{0}=0\left(\left|\psi_{\text {in }, 2}\right\rangle: \mathcal{R}_{3}\right)$ where the density distribution is flatter. Since the ground-state search here is for $\omega_{0} / t_{0}=1$ and $\gamma / t_{0}=2$ which is in the CDW phase one would expect that $\left|\psi_{\mathrm{in}, 1}\right\rangle$ is a better initial guess state than 
$\left|\psi_{\text {in }, 2}\right\rangle$ since $\left|\psi_{\text {in,1 }}\right\rangle$ has already more of the expected structure for a CDW state built in than $\left|\psi_{\text {in, } 2}\right\rangle$.

The main panel of Fig. 7.2(a) shows the development of the subspaceexpansion mixing factor $\alpha$ during the sweeping. One can see how the mixing factor is fixed to one for the first 30 sweeps and then decreases in case of $\mathcal{R}_{1}$ and $\mathcal{R}_{3}$. In $\mathcal{R}_{1}$ the mixing factor drops rapidly to a value close to $10^{-7}$ after the adaption of $\alpha$ is turned on and stays close to that value for the rest of the simulation. The development of $\alpha$ is different in $\mathcal{R}_{2}$ where the mixing factor is allowed to change already after the first sweep. It also drops to a value close to $10^{-7}$ with a small peak around the 40 th sweep. In $\mathcal{R}_{3}$, the mixing factor is again fixed for the first 30 sweeps. After that, it decreases gradually until it also settles at a value close to $10^{-7}$.

In Fig. 7.2(b), the evolution of the energy guess during the sweeping with respect to the best energy guess during the run is plotted:

$$
\delta_{\text {self }} E=\frac{E_{\text {sweep }}-E_{\text {min,run }}}{\left|E_{\text {min,run }}\right|}
$$

where $E_{\text {sweep }}$ is the energy guess in the middle of a specific sweep and $E_{\text {min,run }}$ is the minimal energy guess during the run. This is the energy evolution one can monitor during the DMRG run.

For all three runs the energy eventually settles into a minimum, with random fluctuations below a relative energy difference of $10^{-10}$. In $\mathcal{R}_{1}$ the energy has an initial drop and then does not improve before the adaption of the mixing factor is turned on. When the mixing factor is allowed to change, the energy rapidly drops to its final value. The data for $\mathcal{R}_{2}$ actually looks very similar although the mixing factor is allowed to change right away. In $\mathcal{R}_{3}$ more sweeps are needed for the energy to settle but after 300 sweeps it does and only random fluctuations are seen.

In Fig. 7.2(c) the evolution of the energy guess during the sweeping with respect to the best energy guess between all the runs is plotted:

$$
\delta E=\frac{E_{\text {sweep }}-E_{\text {min }}}{\left|E_{\min }\right|}
$$

where $E_{\text {min }}$ turns out to be the best energy guess of $\mathcal{R}_{1}$.

Figure 7.2(c) reveals that, although the energies in all the runs settle to a certain value, there are still large differences between them. $\mathcal{R}_{3}$ does not get close in energy to $\mathcal{R}_{1}$ and a look at the inset of Fig. 7.2(b) reveals that the electron density in the final state of $\mathcal{R}_{3}$ does not resemble a charge density wave at all. In this case the algorithm got stuck. On the other hand, the electron density in the final state of $\mathcal{R}_{2}$ and $\mathcal{R}_{1}$ are very similar and have 
the form of a nice charge density wave. Nevertheless, the relative energy difference between $\mathcal{R}_{2}$ and $\mathcal{R}_{1}$ in the later sweeps is of the order $10^{-6}$ which is way beyond the random fluctuations of $10^{-10}$.

There are several conclusions from Fig. 7.2. First of all, it becomes clear that the initial guess state has a huge impact on the convergence. Especially in the charge-density wave phase, it becomes hard for the algorithm to move the polarons around due to their increased effective mass. Therefore, the algorithm can get stuck as it happened for $\mathcal{R}_{3}$.

Second, it can be advisable to fix the mixing factor $\alpha$ to a large value in the first sweeps. The energy will then stabilize after a few sweeps and no major improvements will happen because the large $\alpha$ hinders a further reduction of the energy. Only after this stabilization of the energy is seen the mixing factor should be allowed to change.

Last, it becomes clear that judging the quality of the data can be very challenging. Often, it is necessary to perform several runs with the same initial guess state and different simulation parameters to test the quality. Fortunately, it turns out that if the parameters of the Hamiltonian are kept fixed and only the bond dimension is increased from run to run, the algorithm behaves more predictable.

In general, it is not clear if the presented algorithm and especially the outlined adaption strategy of the mixing factor is optimal. There are a lot of simulation parameters that have to be chosen which makes working with the algorithm quite demanding.

\subsection{Test against exact diagonalization}

As a validation that the implementation of the DMRG3S+LBO algorithm provides correct results I will compare data produced by this algorithm with ground-state data from a Lanczos algorithm [39]. The Lanczos data was provided by J. Herbrych from the Wrocław University of Science and Technology [137]. This comparison also serves the purpose of getting a feeling for the characteristics and capabilities of the specific implementation.

In Fig. 7.3, I compare ground-state data for the Holstein model Eq. (3.4) at half filling and for open boundary conditions at system size $L=4$, phonon frequency $\omega_{0} / t_{0}=2$, and coupling strength $\gamma / t_{0}=3$. This parameter set corresponds to the transition region between the metallic and the CDW phase (cf. Ref. [24]). In Fig. 7.3(a), I plot the energy difference between the DMRG estimate for the ground-state energy and the energy obtained by the Lanczos 


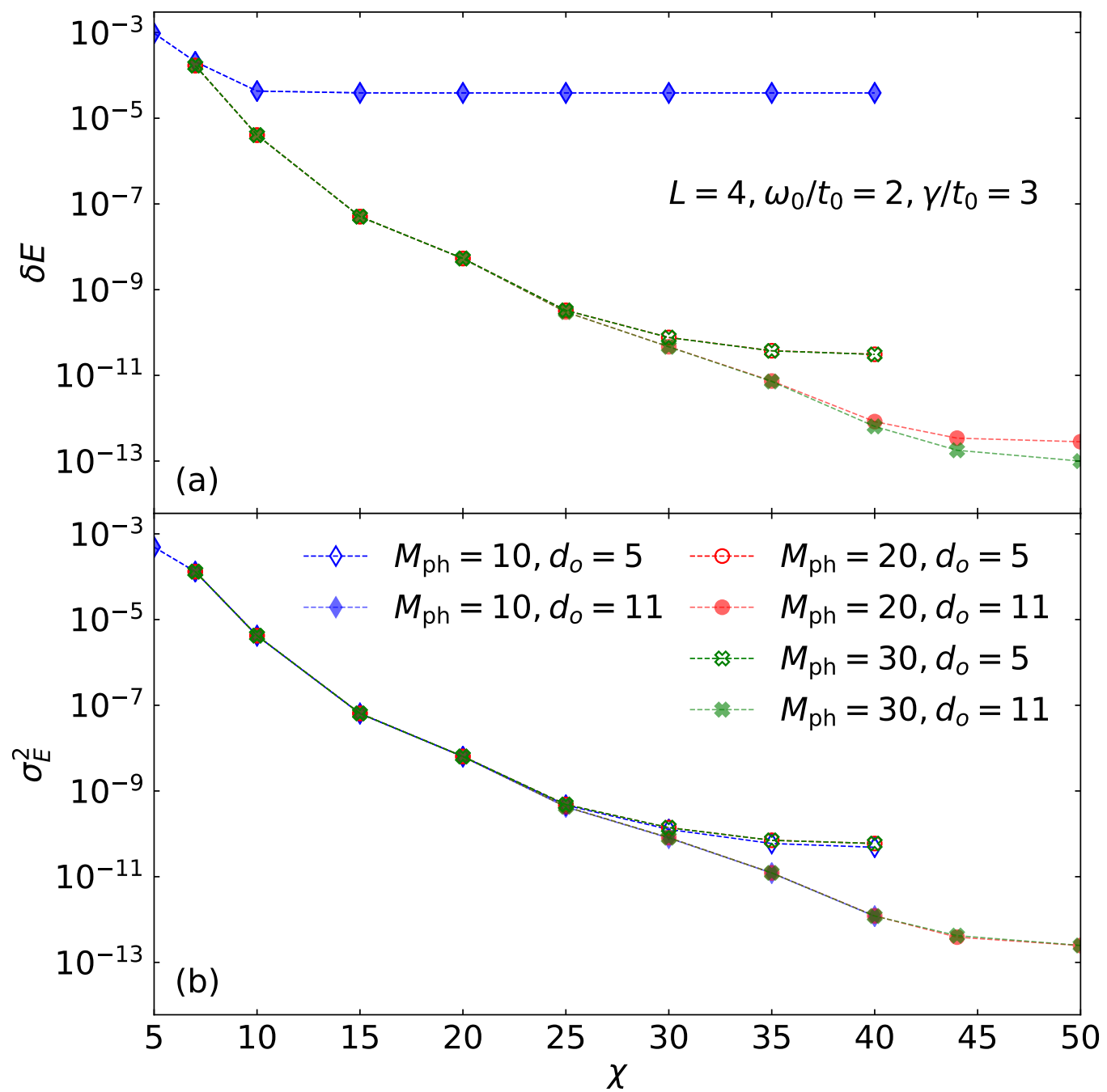

Figure 7.3: (a) Difference between ground-state energy estimate from DMRG3S+LBO and ground-state energy from a Lanczos calculation [137] and (b) variance of the energy plotted against the maximal bond dimension. The different data sets correspond to different maximal phonon numbers $M_{\mathrm{ph}}$ and different numbers of optimal modes $d_{\mathrm{o}}$.

algorithm on the $y$-axis:

$$
\delta E=\frac{E_{\mathrm{DMRG}}-E_{\mathrm{Lz}}}{\left|E_{\mathrm{Lz}}\right|}
$$

where $E_{\text {DMRG }}$ is the energy obtained by the DMRG3S+LBO algorithm and $E_{\mathrm{Lz}}$ is the energy obtained by the Lanczos algorithm. The energy obtained from the Lanczos algorithm was computed with a maximal number of $M_{\mathrm{ph}}=$ 
30 phonons per site and the error of the energy is of the order of the machine precision $\left(<10^{-15}\right)$. In Fig. 7.3(b), the variance of the energy expectation value is plotted on the $y$-axis:

$$
\sigma_{E}^{2}=\frac{\left\langle H^{2}\right\rangle-E_{\mathrm{DMRG}}^{2}}{E_{\mathrm{DMRG}}^{2}} .
$$

The variance can be taken as an estimate for the error in the energy as it gives a measure for the proximity to an eigenstate of the Hamiltonian [138]. On the mutual $x$-axis of the plots, the largest bond dimension $\chi$ in the respective states is plotted.

Six different datasets are plotted in Fig. 7.3. The maximal number of phonons per site is varied from $M_{\mathrm{ph}}=10$ (blue symbols) to $M_{\mathrm{ph}}=20$ (red symbols) to $M_{\mathrm{ph}}=30$ (green symbols). For each $M_{\mathrm{ph}}$, there are two data sets with $d_{\mathrm{o}}=5$ (open symbols) and $d_{\mathrm{o}}=11$ (filled symbols). The larger number of optimal modes $d_{\mathrm{o}}=11$ means that at $M_{\mathrm{ph}}=10$, no truncation is done in the physical dimension. The Lanczos data is computed with $M_{\mathrm{ph}}=30$, as well.

In Fig. 7.3(a), one can observe how the energy converges as a function of the maximal bond dimension $\chi$. It is evident that for $\omega_{0} / t=2$ and $\gamma / t=3$ with $M_{\mathrm{ph}}=10$ the relative distance to the ground-state energy from Lanczos does not decrease below $\delta E \approx 0.3 \times 10^{-4}$. Upon increasing $M_{\mathrm{ph}}$ to 20 and $30 \mathrm{a}$ higher accuracy can be reached. Furthermore, the number of optimal modes plays a role in the convergence. With $d_{\mathrm{o}}=5$ an accuracy of $\delta E \approx 0.3 \times 10^{-10}$ can be reached at the considered parameter set. Upon increasing the number of optimal modes the accuracy is further increased until $\delta E \lesssim 1 \times 10^{-13}$.

The convergence of the variance plotted in Fig. 7.3(b) is quite different. For $d_{\mathrm{o}}=5$ the variance of the energy converges towards $\approx 0.5 \times 10^{-10}$ irrespective of the size of $M_{\mathrm{ph}}$. After increasing $d_{\mathrm{o}}$ to 11 the variance decreases to $\sigma_{E}^{2} \approx 1 \times 10^{-13}$ for all $M_{\mathrm{ph}}$ at the largest bond dimensions. This behavior can be understood by noting that changing $M_{\mathrm{ph}}$ changes the Hilbert space and, therefore, strictly speaking the model one is investigating. Within a certain Hilbert space the algorithm can find states that are very close to being eigenstates of the Hamiltonian. However, the goal is to get results that do not depend on the number of phonons per site any more and are therefore indistinguishable from the results in the limit of infinite $M_{\mathrm{ph}}$ within the desired accuracy. Therefore, the combined results in Fig. 7.3(a) and in Fig. 7.3(b) have to be considered to assess the precision of the calculations.

For example, the results suggest that $M_{\mathrm{ph}}=30$ is actually enough to get an accuracy of $\approx 1 \times 10^{-13}$. The results for $M_{\mathrm{ph}}=20$ and $M_{\mathrm{ph}}=30$ differ only by about $\delta E \approx 0.2 \times 10^{-12}$ for the largest bond dimensions. A further increase of $M_{\mathrm{ph}}$ would not have a noticeable effect at this level of accuracy. 
This expectation was validated by running calculations with $M_{\mathrm{ph}}=40$ (data not shown here).

In general, it turns out that the implementation of DMRG3S+LBO used here is capable of producing reliable results up to an accuracy of about $\delta E \approx$ $10^{-12}$. The saturation of the accuracy can be detected from the fact that the maximal bond dimension that one fixes for a specific DMRG run is not exhausted by the algorithm.

\subsection{Benchmark of DMRG3S+LBO against other DMRG methods}

To demonstrate the capabilities of the DMRG3S+LBO algorithm and to illustrate dependencies on the internal control parameters $M_{\mathrm{ph}}, d_{\mathrm{o}}$, and $\chi \mathrm{I}$ will compare ground-state data for large systems of $L=51$ sites and $N=$ 25 fermions with data from PS-DMRG and PP-DMRG. An odd number of sites was chosen such that a nonzero value of the charge density wave order parameter $\mathcal{O}_{\mathrm{CDW}}$ Eq. (3.19) develops in the charge density wave phase on finite systems. The ground state of a finite system with open boundary conditions in one dimension has to be mirror symmetric with respect to the middle of the system unless this symmetry is explicitly broken. This means that $\mathcal{O}_{\mathrm{CDW}}$ is automatically zero in the ground state of a system with an even number of sites. Choosing an odd length of the system also has the consequence that we do not work at exactly half filling but very close to it.

To get a feeling for how large the maximal phonon number $M_{\mathrm{ph}}$ per site has to be for a given parameter set it is helpful to inspect the exact ground state in the atomic limit Eq. (3.12). The probability for the harmonic oscillator at site $l$ to be in the $m$ th excited state if the site is occupied by an electron is given by:

$$
\begin{aligned}
\mathcal{P}\left(\left\langle b_{l}^{\dagger} b_{l}\right\rangle=m \mid l \in\left\{l_{\mathrm{occ}}\right\}\right) & =\left|{ }_{\mathrm{ph}, l}\left\langle m\left|\mathrm{e}^{-\frac{\gamma^{2}}{2 \omega_{0}^{2}}} \mathrm{e}^{\frac{\gamma}{\omega_{0}} b_{l}^{\dagger}}\right| \emptyset\right\rangle_{\mathrm{ph}, l}\right|^{2} \\
& =\mathrm{e}^{-\frac{\gamma^{2}}{\omega_{0}^{2}}} \frac{\gamma^{2 m}}{\omega_{0}^{2 m} m !}
\end{aligned}
$$

where $|m\rangle_{\mathrm{ph}, l}=1 / \sqrt{m !}\left(b_{l}^{\dagger}\right)^{m}|\emptyset\rangle_{\mathrm{ph}, l}$.

A plot of these probabilities as a function of $m$ for different $\gamma / \omega_{0}$ can be seen in Fig. 7.4. One can observe how the probabilities for larger $m$ rises as the coupling is increased. Of course, this can only be a rough indication on how large the maximal phonon number has to be for a simulation in a certain parameter regime. Close to the atomic limit and particularly in the CDW 


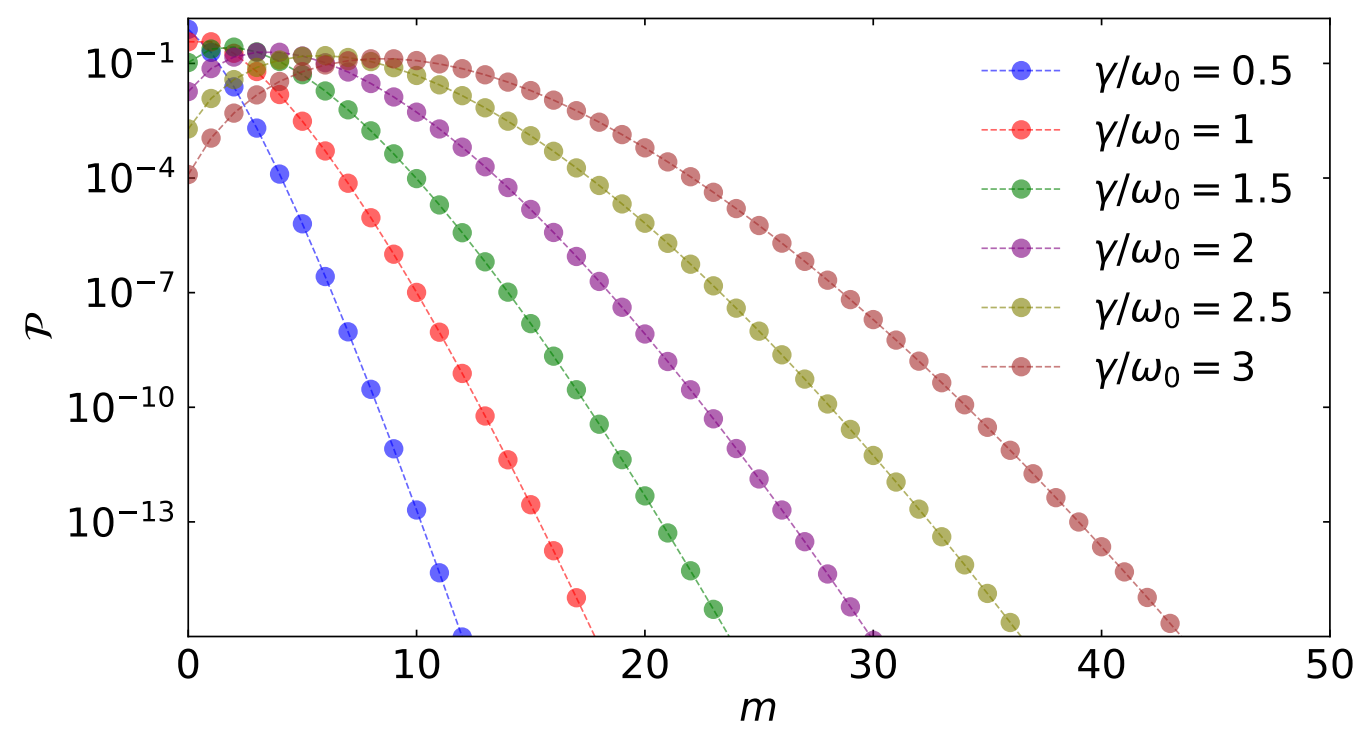

Figure 7.4: Plot of the probabilities for the harmonic oscillator of site $l$ to be in the $m$ th excited state if the site is occupied by an electron Eq. (7.5) as a function of the excitation $m$ for different $\gamma / \omega_{0}$ in the atomic limit $t=0$.

phase one would expect that the estimation of the necessary $M_{\mathrm{ph}}$ based on Eq. (7.5) is quite accurate. Away from these limits, the ground states of Eq. (3.4) do not resemble the ground state in the atomic limit Eq. (3.12) and thus the estimate of $M_{\mathrm{ph}}$ based on Eq. 7.5 might be poor. Therefore, one still has to justify a certain choice of $M_{\mathrm{ph}}$ numerically.

In Figs. 7.5, 7.6, 7.7, 7.8, and 7.9, data obtained with DMRG3S+LBO, PS-DMRG, and PP-DMRG is shown. In the respective panels (a), the relative energy difference is plotted compared to the minimal energy $E_{\min }$ found across all the compared methods. The lack of a more precise ground-state energy to compare to forces one to choose such an ansatz. The relative energy difference $\delta E$ is then calculated by replacing $E_{\mathrm{Lz}}$ by the respective $E_{\text {min }}$ in Eq. (7.3). In the panels (b), the variance of the energy Eq. (7.4) is plotted.

The plots are organized as follows. In the Figs. 7.5, 7.6, and 7.7, data is shown for $\omega_{0} / t=1$ and $\gamma / t=0.5,1.5,2$, respectively. The hopping matrix element and the phonon frequency are equal and the parameter sets correspond to the metallic Tomonaga-Luttinger liquid (TLL) phase, the transition region and the charge-density-wave (CDW) phase, respectively. In Fig. 7.8, data is shown for $\omega_{0} / t=4$ and $\gamma / t=10$ which is on the antiadiabatic side in the CDW phase and in Fig. 7.9, data is shown for $\omega_{0} / t=0.2$ and $\gamma / t=0.6$ which is on the adiabatic side in the the CDW phase.

The maximal bond dimensions in the Figs. 7.5, 7.6, 7.7, 7.8, and 7.9 are 


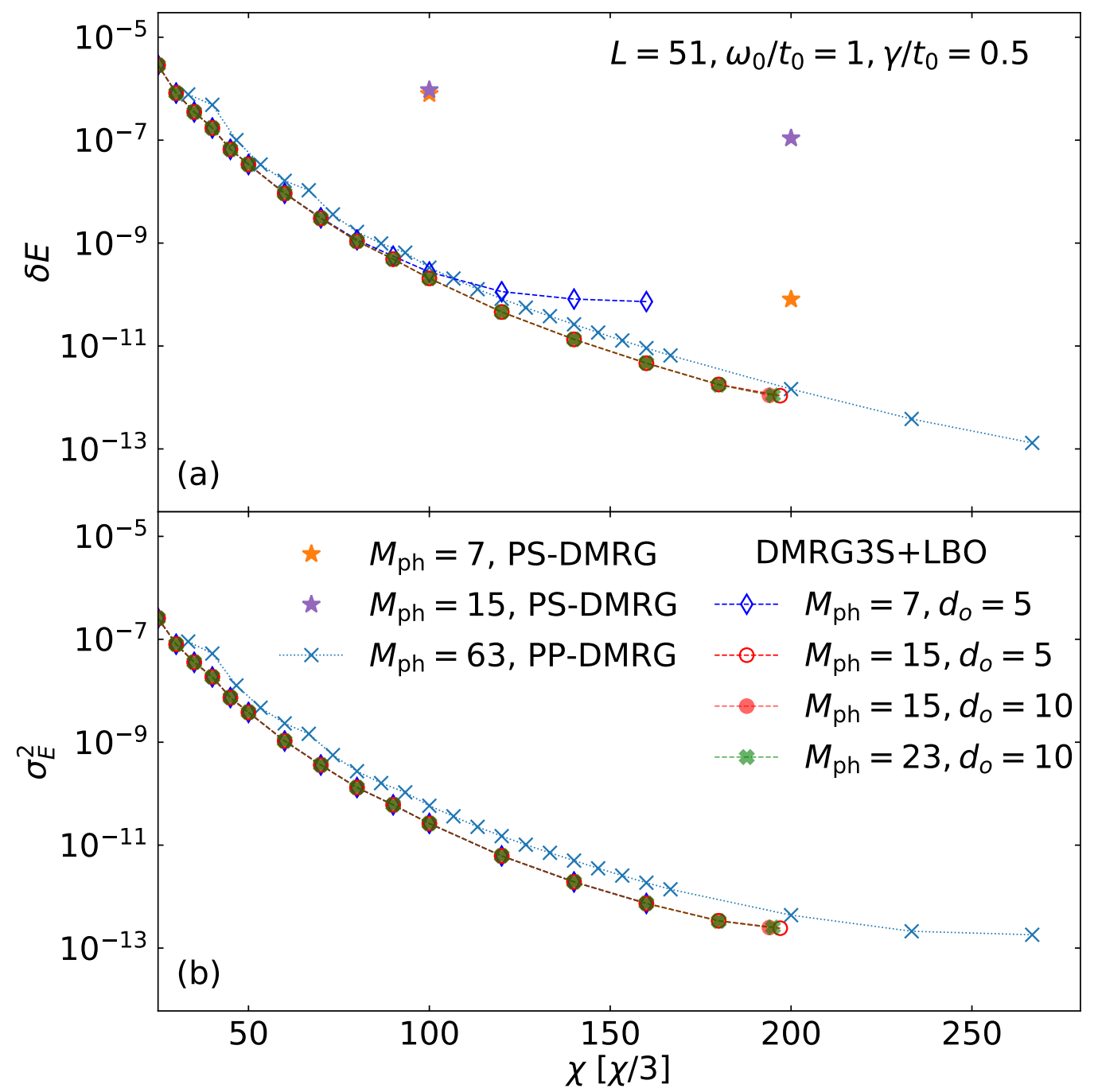

Figure 7.5: TLL phase: (a) Relative energy difference between the lowest energy found by PP-DMRG [133] and the energies found by PS-DMRG [136] (stars), PP-DMRG [133] (crosses), and DMRG3S+LBO (bold symbols), respectively, as a function of the bond dimension. (b) Variance of the energy calculated with PP-DMRG [133] (crosses) and DMRG3S+LBO (bold symbols) as a function of the bond dimension. Calculations done for the Holstein model Eq. (3.4) close to half filling with system size $L=51, N=25$ electrons in the system, $\omega_{0} / t=1$, and $\gamma / t=0.5$. All bond dimensions for the PP-DMRG data are divided by 3 .

rescaled by $3,6,7,10$ and 10 , respectively, in case of the PP-DMRG data. This ad-hoc rescaling is done to fit the data from PS-DMRG, PP-DMRG, 
and DMRG3S+LBO into a single plot for a better comparability. As discussed earlier, the bond dimension in the PP-DMRG method has to be larger for a comparable precision. The entanglement between the physical sites and the phonon bath-sites have to be encoded in the MPS on top of the entanglement between the physical sites. A detailed discussion of the enlarged bond dimension in case of the PP-DMRG method is done in Refs. [37,130].

Metallic TLL phase: $\omega_{0} / \mathbf{t}=1, \gamma / \mathbf{t}=0.5$

In Fig. 7.5(a), we see that the energy in the TLL phase for $\omega_{0} / t=1$ and $\gamma / t=0.5$ converges with growing bond dimension. As anticipated from Fig. 7.4, $M_{\mathrm{ph}}=7$ is, however, not large enough to reach the full precision that is possible with the used implementation of the DMRG3S+LBO algorithm. On the other hand, a larger number of phonons of $M_{\mathrm{ph}}=15$ is sufficient to reach the maximal precision as a further enlargement of $M_{\mathrm{ph}}$ to 23 does not improve the precision. Furthermore, the number of optimal modes per symmetry block of $d_{\mathrm{o}}=5$ is enough for a relative precision of $\delta E \approx 10^{-12}$. This can also be concluded from Fig. 7.5(b) since the variance for $d_{\mathrm{o}}=5$ is decreasing in the whole range of bond dimensions instead of getting stuck at some point (cf. the $d_{\mathrm{o}}=5$ data in Fig. 7.3).

The energies obtained from DMRG3S+LBO, PP-DMRG and PS-DMRG are overall consistent. As expected, the PS-DMRG needs a larger bond dimension to accomplish a similar precision as the DMRG3S+LBO (this is true for all parameter sets analysed in this chapter, cf. Figs. 7.5, 7.6, 7.7, 7.8, and 7.9). Furthermore, the precision gets actually worse when increasing the maximum phonon number in case of the PS-DMRG $\chi=200$ data. This can be explained by the fact that the chain lengthens when $M_{\mathrm{ph}}$ is increased in case of the PS-DMRG. If the additional pseudo sites do not have any occupation, there should be no effect. If, however, the added pseudo sites are occupied, the precision with the same bond dimension can get worse.

Comparing the energies obtained from PP-DMRG with $M_{\mathrm{ph}}=63$ and DMRG3S+LBO with $M_{\mathrm{ph}}=15,23$ in Fig. 7.5(a) one can see that the energies scale similar with the maximal bond dimension if the bond dimensions in the PP-DMRG method are divided by 3. This overall behavior, that a rescaling of the bond dimensions from the PP-DMRG yields a similar scaling of the energy as in the DMRG3S+LBO method can be seen for all parameter sets shown here (cf. Figs. 7.5, 7.6, 7.7, 7.8, and 7.9). 


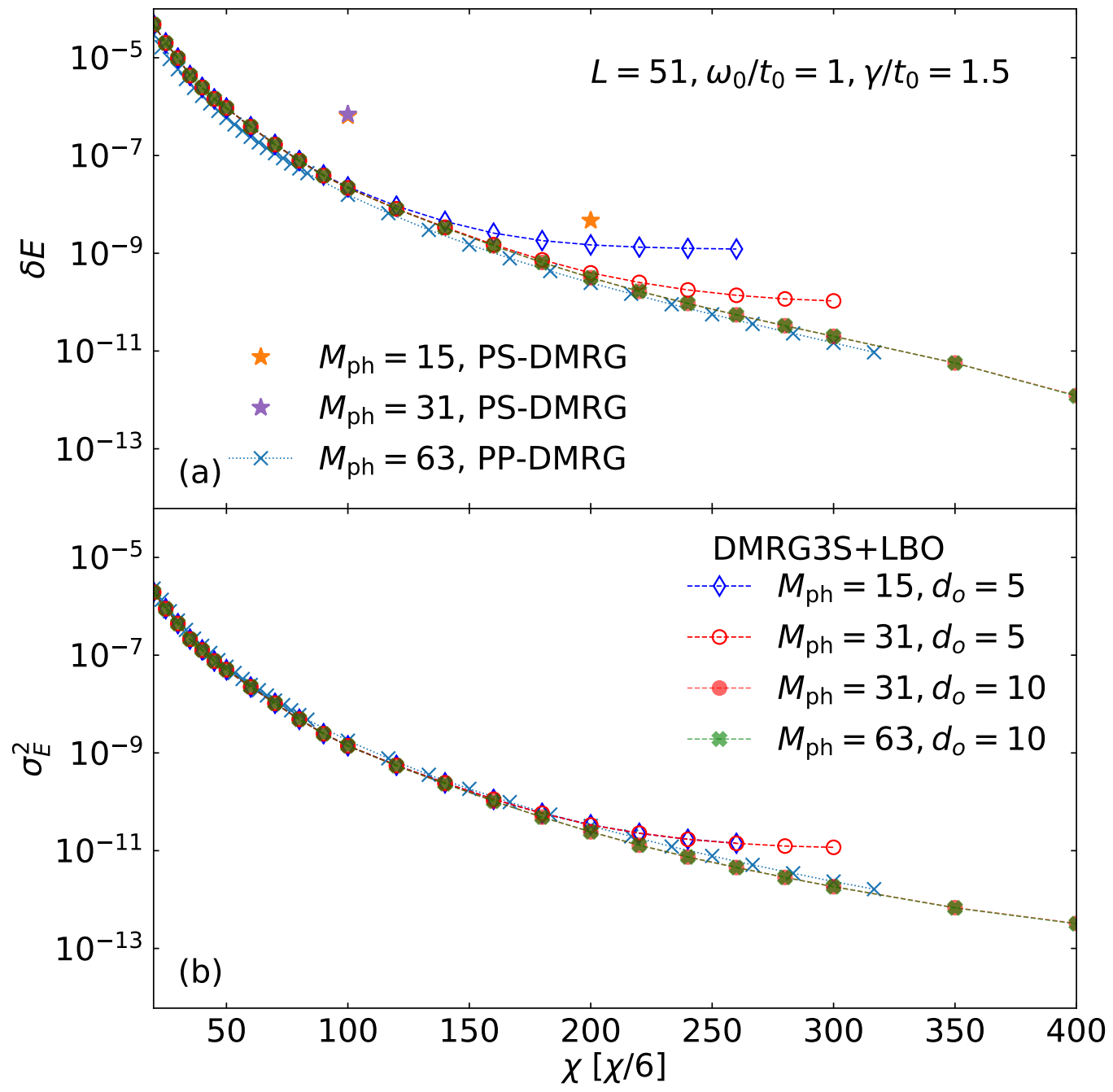

Figure 7.6: Phase transition region: (a) Relative energy difference between the lowest energy found by DMRG3S+LBO and the energies found by PSDMRG [136] (stars), PP-DMRG [133] (crosses), and DMRG3S+LBO (bold symbols), respectively, as a function of the bond dimension. (b) Variance of the energy calculated with PP-DMRG [133] (crosses) and DMRG3S+LBO (bold symbols) as a function of the bond dimension. Calculations done for the Holstein model Eq. (3.4) close to half filling with system size $L=51, N=25$ electrons in the system, $\omega_{0} / t=1$, and $\gamma / t=1.5$. All bond dimensions for the PP-DMRG data are divided by 6 .

Phase transition region: $\omega_{0} / t=1, \gamma / t=1.5$

In Fig. 7.6(a), energies are compared close to the phase boundary between the TLL phase and the CDW phase for $\omega_{0} / t=1$ and $\gamma / t=1.5$. It is evident 
that a larger bond dimension is needed in all methods to reach a similar precision compared to the TLL phase (Fig. 7.5) which is expected close to the phase boundary [115]. Combining the information from Fig. 7.6(a) and (b), one can conclude that $M_{\mathrm{ph}}=15$ and $d_{\mathrm{o}}=5$ is not enough to reach the maximum precision with DMRG3S+LBO. However, $M_{\mathrm{ph}}=31$ and $d_{\mathrm{o}}=10$ is sufficient.

\section{Insulating CDW phase: $\omega_{0} / \mathbf{t}=1, \gamma / \mathbf{t}=\mathbf{2}$}

In Fig. 7.7, data is shown for $\omega_{0} / t=1$ and $\gamma / t=2$ which corresponds to the CDW phase. The bond dimensions needed here are smaller than the ones needed in Figs. 7.5 and 7.6 to reach a similar precision which can be attributed to the existence of an energy gap between the ground state and the excitation spectrum [115]. As discussed in Sec. 4.1.2, these conditions lead to an effective representation of the ground state by matrix product states. It is evident that $d_{\mathrm{o}}=5$ is not sufficient. On the other hand, $M_{\mathrm{ph}}=31$ and $d_{\mathrm{o}}=10$ is adequate for the DMRG3S+LBO method and a further increase of $M_{\mathrm{ph}}$ is not necessary.

Insulating CDW phase - antiadiabatic: $\omega_{0} / \mathbf{t}_{0}=4, \gamma / \mathbf{t}_{0}=10$

Figure 7.8 shows the convergence of the energy and energy variance in the antiadiabatic region of the CDW phase at $\omega_{0} / t_{0}=4$ and $\gamma / t_{0}=10$. First of all, one notices that the bond dimensions here are again much smaller compared to the previous datasets. With the increase of $\omega_{0} / t_{0}$ one approaches the atomic limit where the ground state takes the form Eq. (3.12) which is a product state and, therefore, the small bond dimensions come as no surprise.

Furthermore, the convergence of the energy with respect to the bond dimension appears to be not as smooth in case of the DMRG3S+LBO method compared to the other datasets (cf. Figs. 7.5, 7.6, and 7.7). It turns out that it is quite challenging to get the DMRG3S+LBO method to converge to the correct ground state in the antiadiabatic region. In this limit, the polarons are very small [21] and their effective mass is quite high. Therefore, the energy penalty for having two polarons next to each other is not as high compared to the other datasets and it can easily happen that the algorithm gets stuck in very stable states away from the ground state.

\section{Insulating CDW phase - adiabatic: $\omega_{0} / \mathrm{t}=0.2, \gamma / \mathrm{t}=0.6$}

The last figure of this series is Fig. 7.9 where the energy and energy variance is plotted in the adiabatic region of the CDW phase at $\omega_{0} / t=0.2$ and $\gamma / t=0.6$. Here again the situation is similar to the one at $\omega_{0} / t=1$. One can clearly 


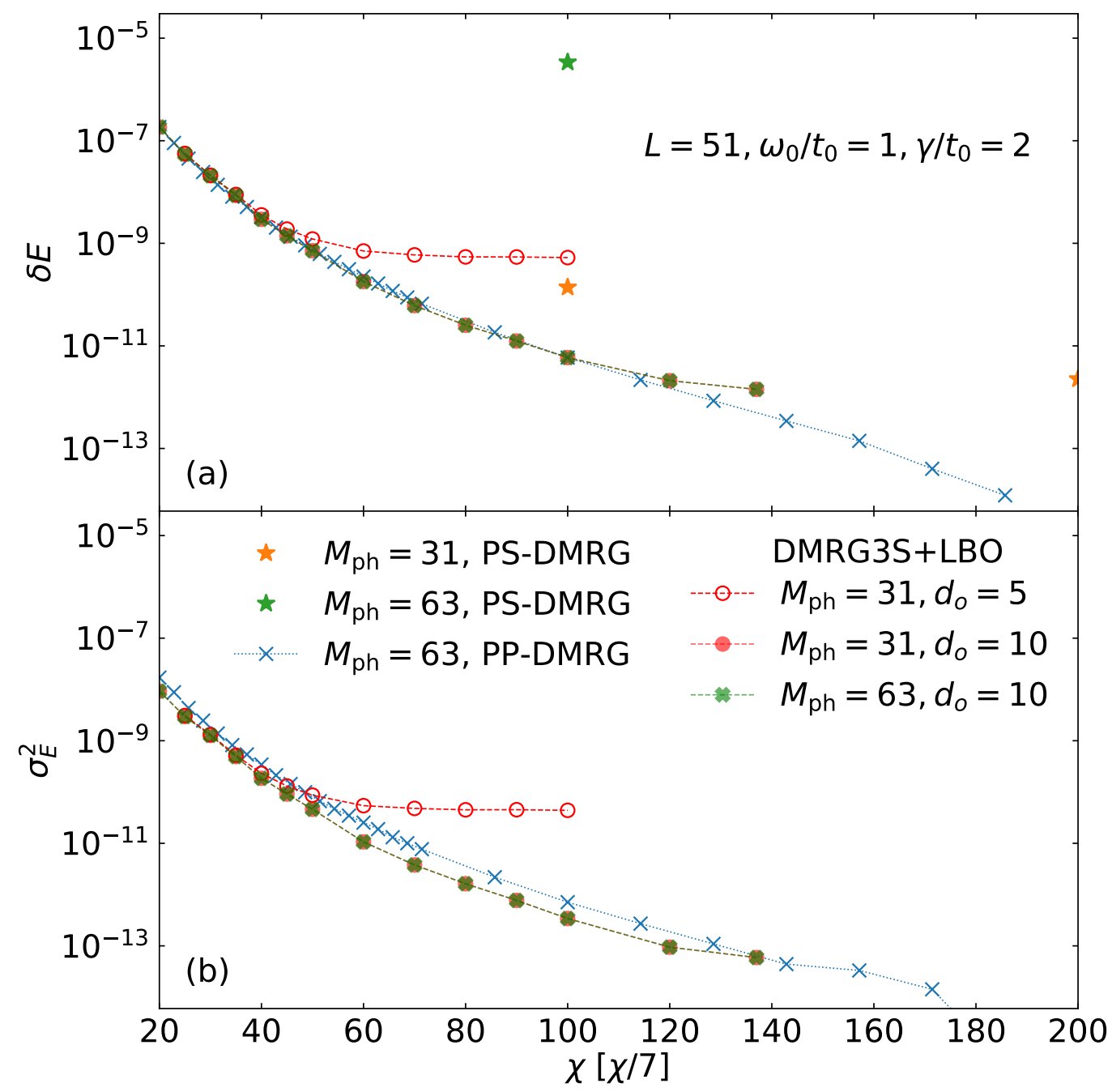

Figure 7.7: CDW phase: (a) Relative energy difference between the lowest energy found by PP-DMRG [133] and the energies found by PS-DMRG [136] (stars), PP-DMRG [133] (crosses), and DMRG3S+LBO (bold symbols), respectively, as a function of the bond dimension. (b) Variance of the energy calculated with PP-DMRG [133] (crosses) and DMRG3S+LBO (bold symbols) as a function of the bond dimension. Calculations done for the Holstein model Eq. (3.4) close to half filling with system size $L=51, N=25$ electrons in the system, $\omega_{0} / t=1$, and $\gamma / t=2$. All bond dimensions for the PP-DMRG data are divided by 7 .

see that one needs to converge in both the maximal phonon number per site $M_{\mathrm{ph}}$ and the number of optimal modes $d_{\mathrm{o}}$ to reach the maximal precision possible with DMRG3S+LBO. This is also the only one of the considered 
cases where $M_{\mathrm{ph}}=31$ is clearly not enough to get the full precision and one needs to increase the maximal phonon number beyond that.

\section{Observables}

To conclude this chapter I compare the expectation value of the displacement of the oscillators at ever site $\sqrt{2 \omega_{0}}\left\langle X_{l}\right\rangle=\left\langle b_{l}^{\dagger}+b_{l}\right\rangle$ calculated in the ground states from PP-DMRG and DMRG3S+LBO in Fig. 7.10. In Fig. 7.10(a), the displacement is plotted for the three different parameter sets $\omega_{0} / t=1$ and $\gamma / t=0.5,1.5,2$. The results from the DMRG3S+LBO method are plotted in open symbols and the results obtained from the PP-DMRG method are plotted as black filled symbols. The results in Fig. 7.10(a) correspond to the highest bond dimension results in Figs. 7.10(b),(c),(d), respectively. The data from both methods coincide nicely. The displacements have very little structure and are quite small in the TLL phase at $\omega_{0} / t=1$ and $\gamma / t=0.5$. For $\omega_{0} / t=1$ and $\gamma / t=1.5$ close to the phase transition more structure can be seen. In the CDW phase at $\omega_{0} / t=1$ and $\gamma / t=2$, a charge density wave is formed which is accompanied by a large displacement on all sites occupied by an electron and almost no displacement for all empty sites. Furthermore, small boundary effects can be seen in the results from the CDW phase.

In the Figs. 7.10(b),(c),(d) the difference between the displacements calculated by DMRG3S+LBO and PP-DMRG is plotted:

$$
\Delta_{\left\langle b_{l}^{\dagger}+b_{l}\right\rangle}=\left|\left\langle b_{l}^{\dagger}+b_{l}\right\rangle_{\mathrm{DMRG} 3 \mathrm{~S}+\mathrm{LBO}, \chi}-\left\langle b_{l}^{\dagger}+b_{l}\right\rangle_{\mathrm{PP}-\mathrm{DMRG}, \chi^{\prime}}\right|,
$$

where $\left\langle b_{l}^{\dagger}+b_{l}\right\rangle_{\text {DMRG3S+LBO, } \chi}$ and $\left\langle b_{l}^{\dagger}+b_{l}\right\rangle_{\text {PP-DMRG, } \chi^{\prime}}$ are the displacements obtained with DMRG3S+LBO and PP-DMRG in a state with a certain bond dimension $\chi, \chi^{\prime}$ respectively. The bond dimensions were chosen such that they are of the same size if the bond dimension used in the PP-DMRG method is divided by 3 in Fig. 7.10(b), by 6 in Fig. 7.10(c), and by 7 in Fig. $7.10(d)$.

As expected, the difference between the calculated displacements decreases as the bond dimensions are increased. The smallest differences are seen for the largest bond dimensions at $\gamma / t=0.5$ in Figs. 7.10(b). In Figs. 7.10(c) at $\gamma / t=1.5$ the differences do not decrease as far. Especially between the two largest bond dimensions there is barely any improvement visible. The lower precision for these parameters was expected since also the energies are less precise (cf. Fig. 7.6). In Figs. 7.10(d) for $\gamma / t=2$, there is again a reduction of the differences between the methods as the bond dimension for both of them is increased. 


\subsection{Summary}

This chapter was devoted to the test of the DMRG3S+LBO method and a comparison to the PP-DMRG method. To this end, ground-state data was obtained for the half-filled 1d Holstein model in different regions of the phase diagram and benchmarked against results calculated with PS-DMRG. Overall, the DMRG3S+LBO and the PP-DMRG are equally capable of producing very precise results for all parameter sets considered here, both with respect to ground-state energies and observables.

Especially close to the phase boundary, the DMRG3S+LBO benefits from its ability to represent the ground state with a smaller bond dimension compared to the PP-DMRG results at the same precision. This advantage comes at the cost of a more delicate handling of the method. The algorithm is very sensitive to the initial state and the management of the subspace expansion mixing factor, which can make it hard to get it converged. Furthermore, convergence with respect to the number of optimal modes $d_{\mathrm{o}}$ has to be checked in addition to the convergence with respect to the bond dimension.

The PP-DMRG is conceptually simpler than the DMRG3S+LBO. A standard two-site solver can be used in the DMRG and the bond dimension is the sole control parameter for convergence. 


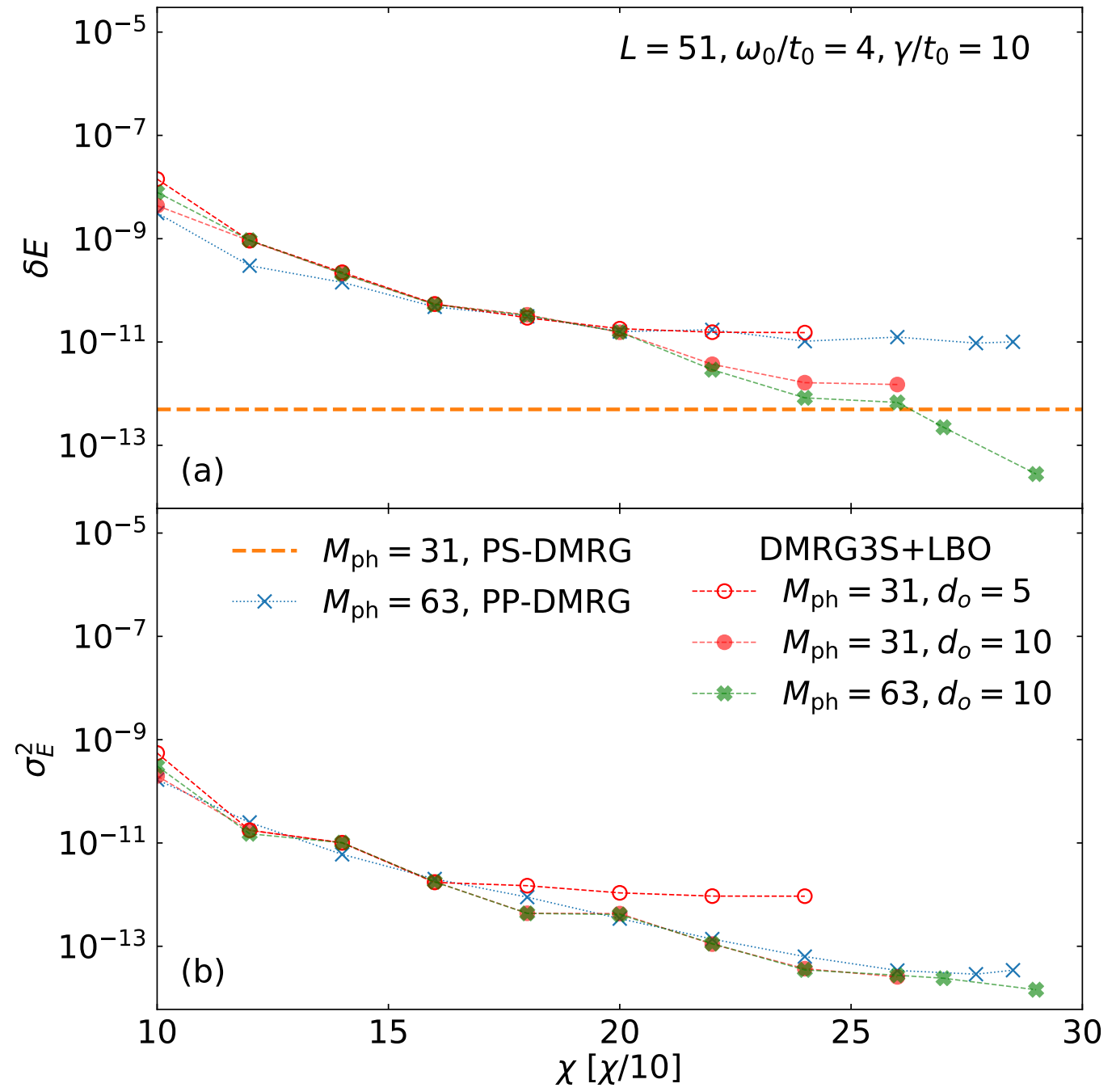

Figure 7.8: CDW phase - antiadiabatic: (a) Relative energy difference between the lowest energy found by DMRG3S+LBO and the energies found by PP-DMRG [133] (crosses), and DMRG3S+LBO (bold symbols), respectively, as a function of the bond dimension. The orange dashed line is the relative energy difference calculated by PS-DMRG [136] with a bond dimension of $\chi=100$. (b) Variance of the energy calculated with PP-DMRG [133] (crosses) and DMRG3S+LBO (bold symbols) as a function of the bond dimension. Calculations done for the Holstein model Eq. (3.4) close to half filling with system size $L=51, N=25$ electrons in the system, $\omega_{0} / t_{0}=4$, and $\gamma / t_{0}=10$. All bond dimensions for the PP-DMRG data are divided by 10 . 


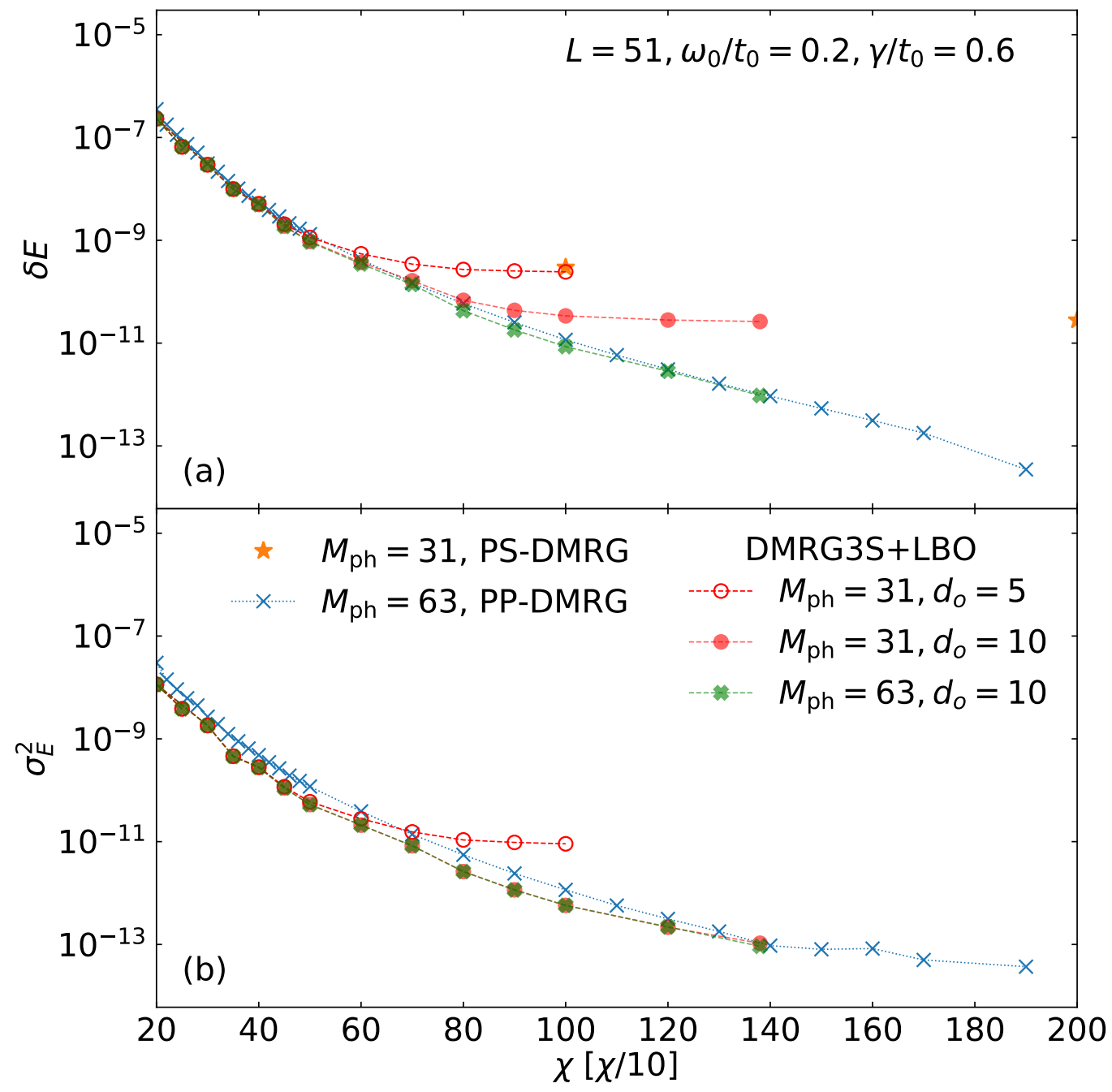

Figure 7.9: CDW phase - adiabatic: (a) Relative energy difference between the lowest energy found by PP-DMRG [133] and the energies found by PSDMRG [136] (stars), PP-DMRG [133] (crosses), and DMRG3S+LBO (bold symbols), respectively, as a function of the bond dimension. (b) Variance of the energy calculated with PP-DMRG [133] (crosses) and DMRG3S+LBO (bold symbols) as a function of the bond dimension. Calculations done for the Holstein model Eq. (3.4) close to half filling with system size $L=51$, $N=25$ electrons in the system, $\omega_{0} / t=0.2$, and $\gamma / t=0.6$. All bond dimensions for the PP-DMRG data are divided by 10 . 


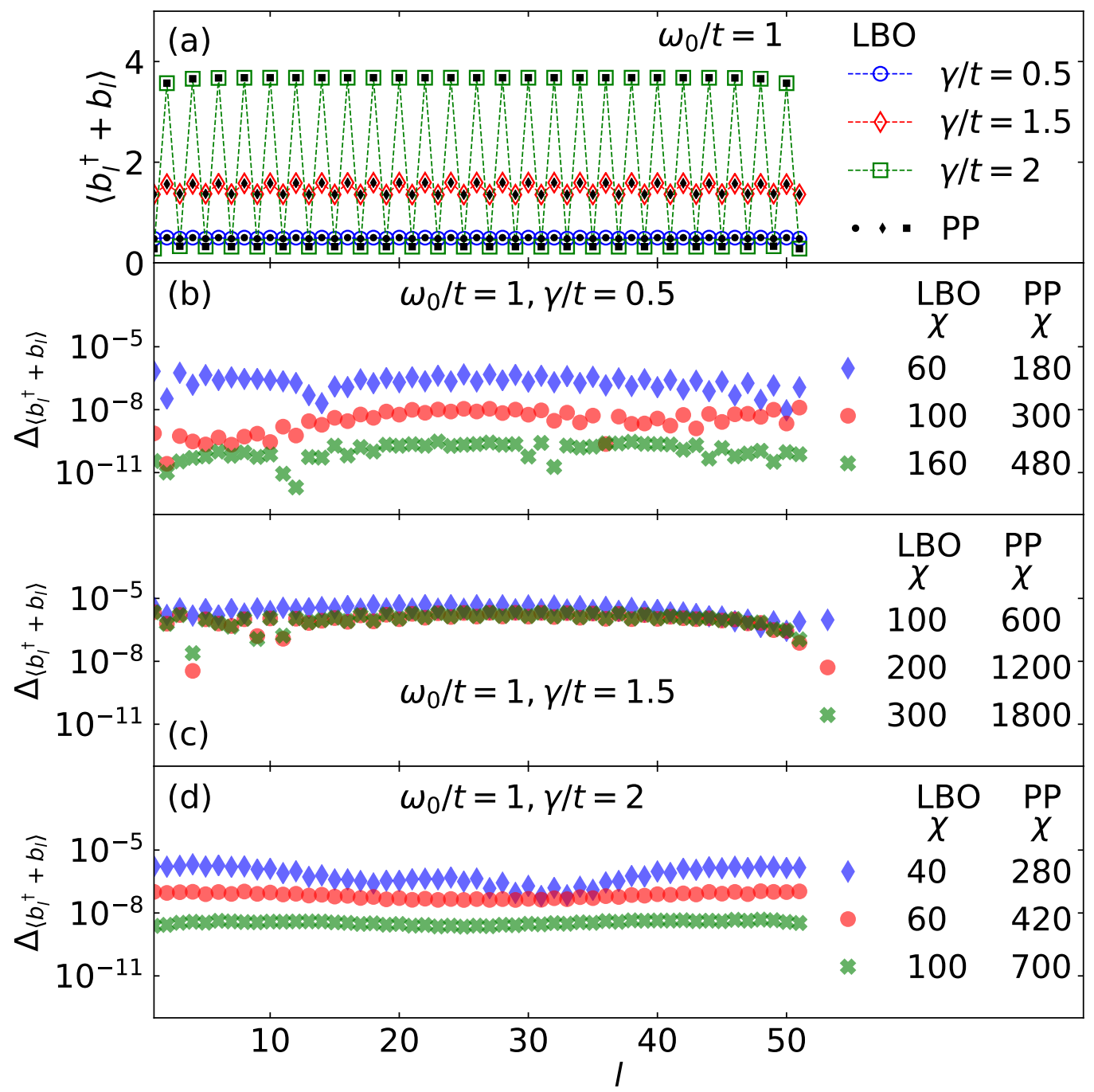

Figure 7.10: (a) Expectation value of the oscillator displacement $\left\langle b_{l}^{\dagger}+b_{l}\right\rangle$ at every site for $L=51, N=25, \omega_{0} / t=1, \gamma / t=0.5,1.5,2$ calculated with DMRG3S+LBO (open symbols) and PP-DMRG [133] (filled symbols). (b) - (d) Difference between the oscillator displacements calculated with DMRG3S+LBO and PP-DMRG [133] for different maximal bond dimensions $\chi$. (b) $\omega_{0} / t=1, \gamma / t=0.5$, (c) $\omega_{0} / t=1, \gamma / t=1.5$, (d) $\omega_{0} / t=1, \gamma / t=2$. 


\section{Chapter 8}

\section{Charge-density-wave melting in the Holstein model}

As we have seen in Ch. 2, modeling solid-state materials can be a challenging task for theoreticians. Here the dynamics in the Holstein model as an example for an electron-phonon coupled system is studied. The study of such model systems can help to understand experiments on ultrafast dynamics in solids.

In these types of experiments, energy is deposited in the material by an external excitation and then the response of the sample is monitored over time. A common setup are so called pump-probe experiments where the sample is excited by a strong laser pulse, the pump pulse. After a waiting time a second laser is shone on the sample to probe the response. By varying the time delay between pump and probe pulse the course of the response in time can be recorded [10].

Due to resonance effects, different degrees of freedom can be targeted for the deposition of the energy. Often the laser pulse primarily couples to the electrons of the systems. Then the electrons couple to each other and to the lattice degrees of freedom, such that after some time the system relaxes back to a steady state. A wide variety of phenomena have been found in these types of experiments such as photoinduced phase transitions [17], accessing metastable states [16], or the melting of CDW or antiferromagnetic order $[13,14,139]$.

On the theoretical side, the challenge is to understand different decay channels and timescales of the relaxation. In this context, the Holstein model is appealing since it features few free parameters which holds the chance to understand microscopic phenomena in detail. Furthermore, it hosts polarons [21] and at half filling a phase transition between a metallic and insulating CDW phase [22-24] which are typical phenomena in electron-phonon coupled systems. 


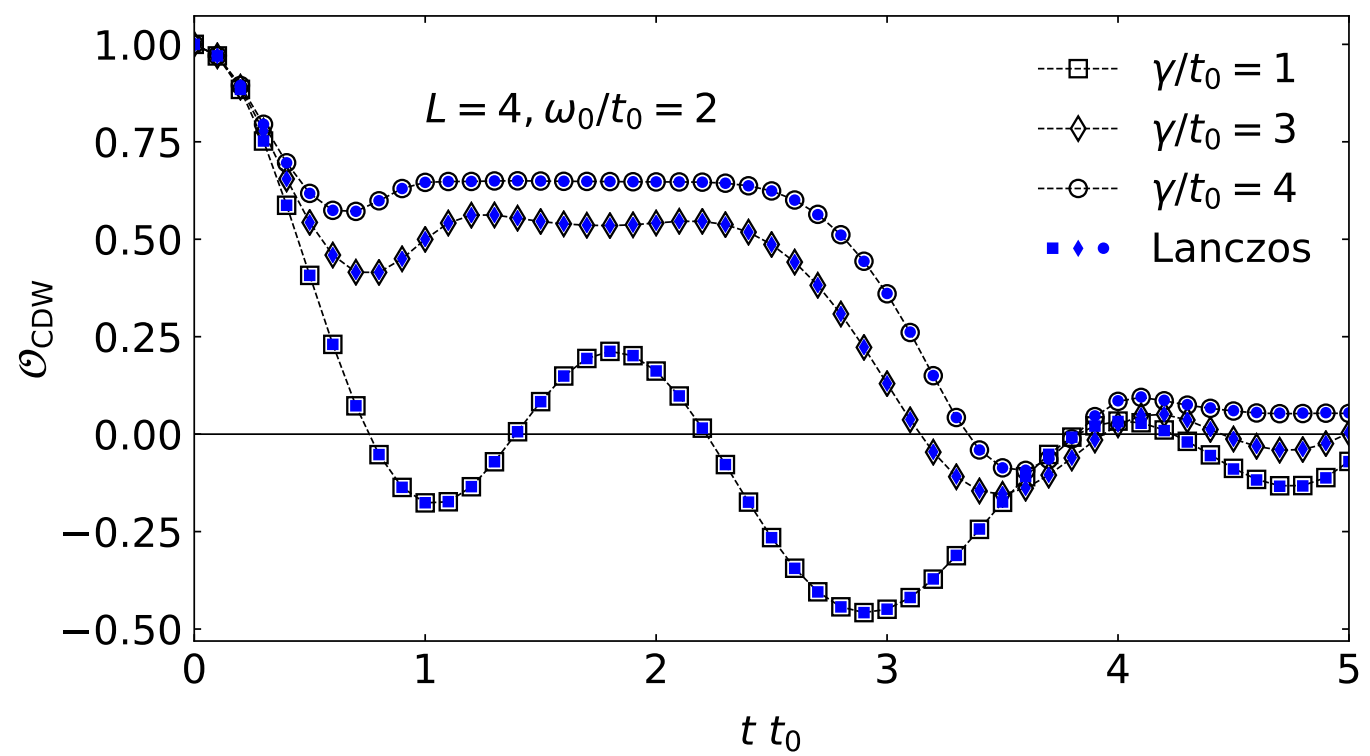

Figure 8.1: Order parameter $\mathcal{O}_{\mathrm{CDW}}$ as a function of time when starting from the state $|1,0,1,0\rangle_{\mathrm{el}}|\emptyset\rangle_{\mathrm{ph}} . L=4, N=2, \omega_{0} / t_{0}=2$ and $\gamma / t_{0}=1,3,4$. Open symbols are TEBD-LBO data with $\delta t t_{0}=0.025, \Delta_{\text {trunc }}=\Delta_{\text {loc }}=$ $10^{-7}$ and $M_{\mathrm{ph}}=15,30,40$, respectively. For clarity, only every fourth data point computed with TEBD-LBO is shown. Small filled symbols are timedependent Lanczos data [137].

To understand the dynamics in the Holstein model one should note that only the electrons can move around in the system. If phonons travel through the system they can do so only by being absorbed by an electron and then reemitted by it at another location.

In this chapter, I will recapitulate the results of Ref. [54]. The data shown here features slightly larger system sizes, however, the conclusions from Ref. [54] do not change. A discussion of the local harmonic oscillators movement in phase space will complement the already existing analysis.

The study presented here focuses on electron-phonon coupling as the sole channel for relaxation and, thus, can be seen as complementary to the work by Hashimoto and Ishihara [57]. They study the melting of charge order as a result of an optical excitation in a system with dominating nearest neighbor electron-electron interaction. The overall effect of an additional weak coupling to phonons is a slowing down of the dynamics due to an increased effective mass of the solitons that cause the melting. 
As in Ch. 7 odd system sizes are used to pin the charge density wave in the CDW ground states and open boundary conditions are considered. For the time evolution the TEBD with local basis optimization (cf. Sec. 5.1) is used. The time step is set to $\delta t t_{0}=0.025$ and the maximal truncation error in both the bond dimension and the optimal modes is set to $10^{-7}$. The bond dimension is then no further restricted and always stays below 2000 for all the simulations below.

The maximal times that are shown in the plots is choses such that finitesize effects do not affect the behavior at the end of the simulations qualitatively (see App. A). At later times reflections from the boundaries would become visible in the data. Furthermore, the bond dimensions then grow above 2000 which makes calculations prohibitively expensive in this case and, therefore, enlarging the system size would not give more information. It was checked that errors due to the size of the time steps, truncation errors and maximal phonon numbers per site are not visible on the scale of the figures below.

As an illustration of the accuracy, TEBD-LBO data is compared to data obtained from a Lanczos time evolution [140] for a small system size of $L=4$ in Fig. 8.1. The Lanczos data was again provided by J. Herbrych from the Wrocław University of Science and Technology [137]. In the Lanczos time evolution the time step is $\delta t t_{0}=0.01, M_{\mathrm{Lz}}=20$ Lanczos steps are used, and the maximum phonon number is set to $M_{\mathrm{ph}}=30$. In the initial state, every second site is occupied by a fermion and no phonons are present $|1,0,1,0\rangle_{\mathrm{el}}|\emptyset\rangle_{\mathrm{ph}}$. The phonon frequency is $\omega_{0} / t_{0}=2$ and three different electron-phonon couplings of $\gamma / t_{0}=1,3,4$ are used. In the TEBD-LBO the maximum phonon number is set to $M_{\mathrm{ph}}=15,30,40$. On the scale of the plot, no differences between the TEBD-LBO data and the Lanczos data are visible.

\subsection{Bare charge-density-wave melting}

In the first initial state that is considered to study charge-density-wave melting, every second site is occupied by a fermion without having any phonons in the system. The state is referred to as bare CDW state (BCDW) and takes the form:

$$
|\mathrm{BCDW}\rangle=\left[\prod_{l=1}^{(L-1) / 2} c_{2 l}^{\dagger}\right]|\emptyset\rangle_{\mathrm{el}}|\emptyset\rangle_{\mathrm{ph}} .
$$

The time evolution is done according to the Hamiltonian of the Holstein model, Eq. (3.4), with $\omega_{0} / t_{0}=2$ and $\gamma / t_{0}=1,3,4$. The position of the three 


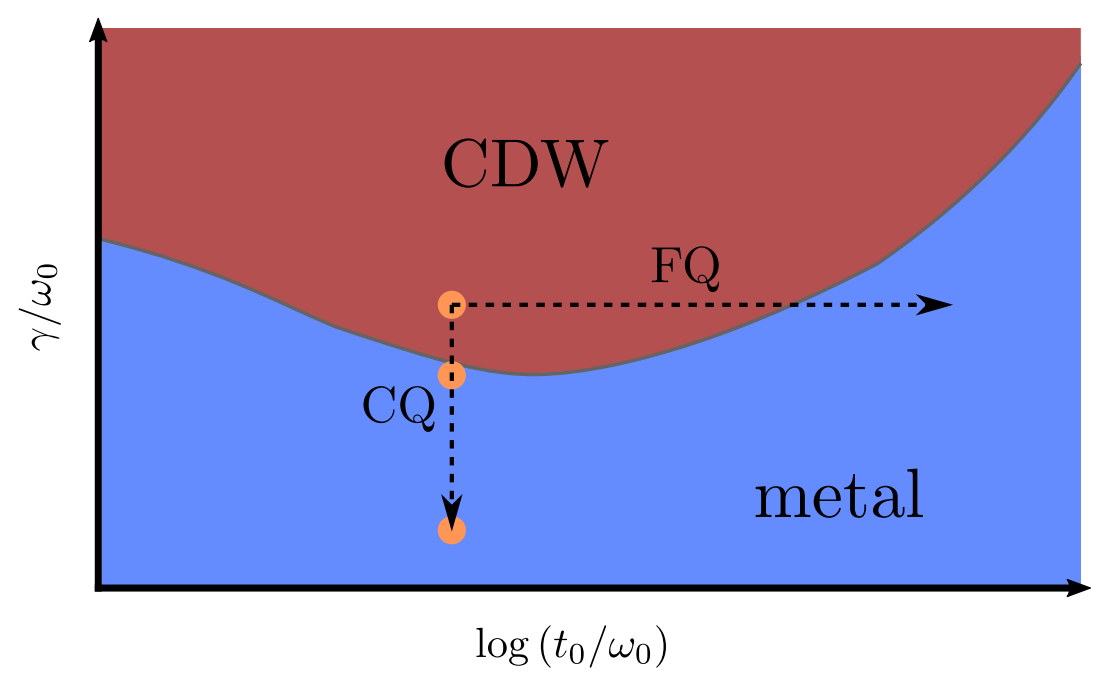

Figure 8.2: Phase diagram of the Holstein model at half filling sketched from the results in Refs. [23,24] (cf. Sec. 3.2). The orange dots represent the parameter sets that are considered for the BCDW and DCDW melting in Secs. 8.1 and 8.2, respectively. The arrows represent the frequency quench (FQ) and coupling quench (CQ) discussed in Sec. 8.3.

parameter sets in the phase diagram of the Holstein model at half filling are roughly marked in Fig. 8.2 as orange dots.

To track the CDW melting the CDW order parameter $\mathcal{O}_{\mathrm{CDW}}$, Eq. (3.19), is plotted in Fig. 8.3(a) as a function of time in units of the hopping matrix element $t_{0}$. As a comparison the time evolution of a BCDW in the thermodynamic limit without coupling to phonons is considered. In this case, the time evolution of $\mathcal{O}_{\mathrm{CDW}}$ follows [141]:

$$
\mathcal{O}_{\mathrm{CDW}, \gamma=0}=J_{0}\left(4 t t_{0}\right)
$$

where $J_{0}$ is the zeroth-order Bessel function of the first kind (see eg. [141]). It is plotted as black dots in Fig. 8.3(a).

For the smallest coupling $\gamma / t_{0}=1$ the order parameter, starting out at $\mathcal{O}_{\mathrm{CDW}}=1$, tends to zero with damped oscillations around that value. It roughly follows the $\gamma=0$ curve until $t t_{0} \approx 3$ and after that the frequency of the oscillations is still similar to the ones at $\gamma=0$. It is evident that the time evolution of $\mathcal{O}_{\mathrm{CDW}}$ is mostly controlled by the hopping matrix element $t_{0}$ with moderate alterations due to the coupling to the phonons.

In contrast, the progression of the order parameter is strongly altered in case of the larger couplings $\gamma / t_{0}=3$ and 4 . After an initial drop of $\mathcal{O}_{\mathrm{CDW}}$, it stabilizes at $\approx 0.6$ between $t t_{0} \approx 1$ and $t t_{0} \approx 2.5$ for $\gamma / t_{0}=4$. Then 


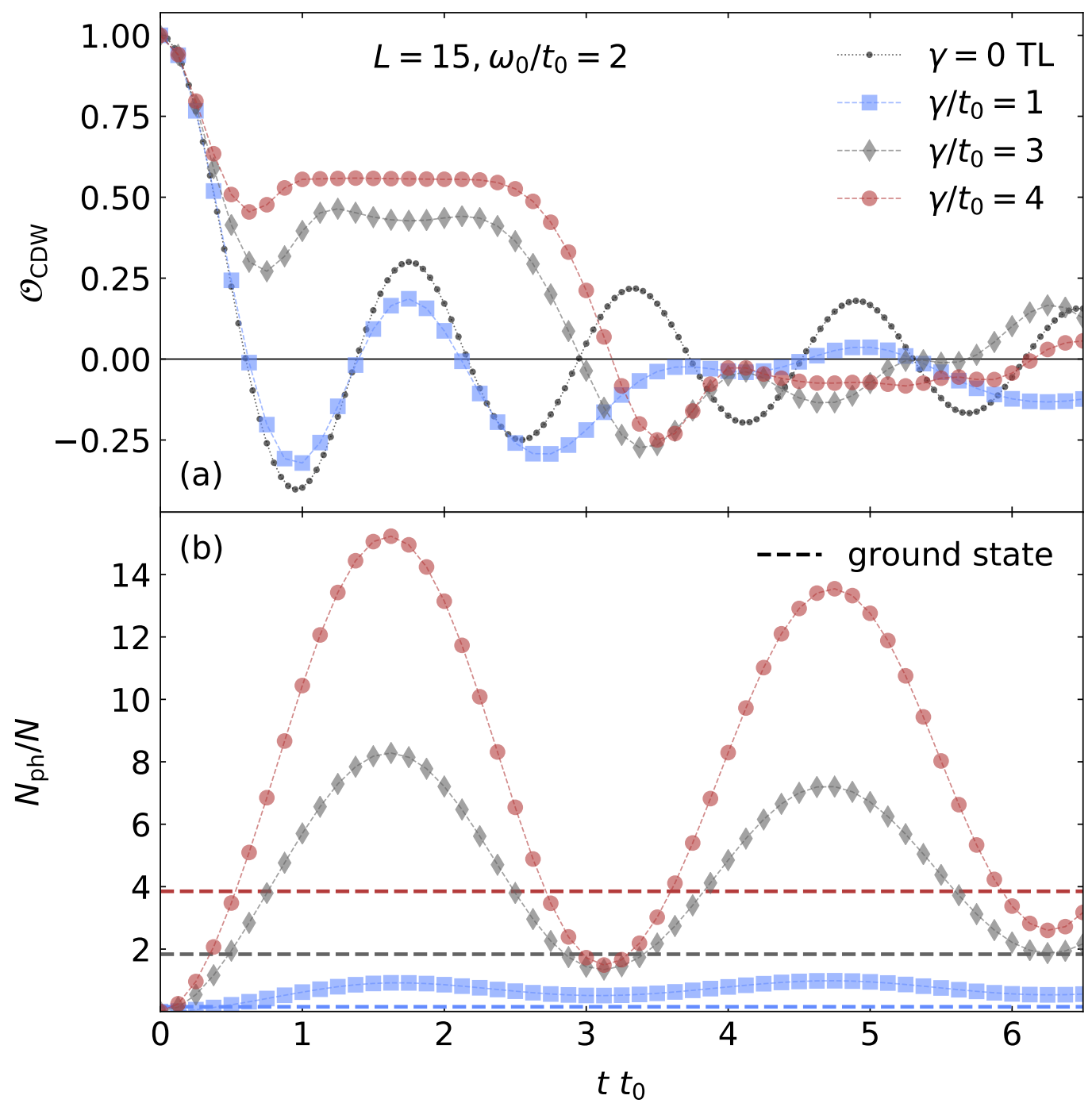

Figure 8.3: Bare CDW melting: (a) order parameter $\mathcal{O}_{\mathrm{CDW}}$ (b) number of phonons in the system $N_{\mathrm{ph}} / N$ as a function of time. $L=15, N=7$, $\omega_{0} / t_{0}=2$ and $\gamma / t_{0}=1,3,4 . M_{\mathrm{ph}}=15,30,40$. For clarity, only every fifth data point computed with TEBD-LBO is shown. The small black dots in (a) correspond to exact analytical results for $\gamma=0$ in the thermodynamic limit [141]. The dashed lines in (b) mark the phonon numbers in the ground state at the respective parameters.

the order parameter changes again until it plateaus around $\approx-0.1$ between $t t_{0} \approx 4.2$ and $t t_{0} \approx 5.8$. At least the first plateau of $\mathcal{O}_{\mathrm{CDW}}$ can similarly be observed for $\gamma / t_{0}=3$, although, in this case the plateau appears at a smaller value of $\mathcal{O}_{\mathrm{CDW}}$ and is shorter in time. 


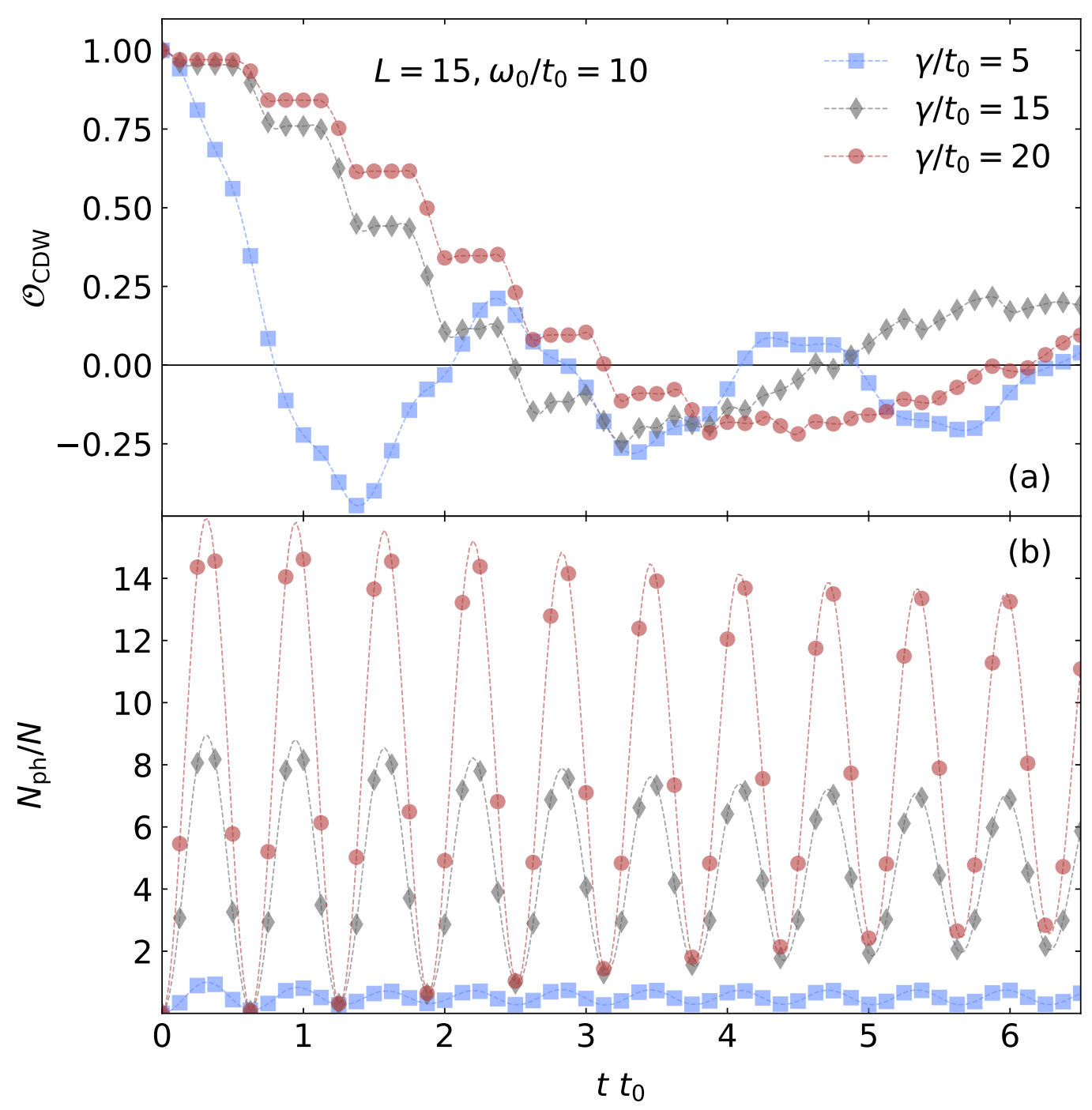

Figure 8.4: Bare CDW melting: (a) order parameter $\mathcal{O}_{\mathrm{CDW}}$ (b) number of phonons in the system $N_{\mathrm{ph}} / N$ as a function of time. $L=15, N=7$, $\omega_{0} / t_{0}=10$ and $\gamma / t_{0}=5,15,20 . M_{\mathrm{ph}}=15,30,40$. For clarity, only every fifth data point computed with TEBD-LBO is shown.

The plateaus in the order parameter are related to oscillations of the number of phonons in the system plotted in Fig. 8.3(b). At the start, no phonons are present in the system and the electrons can move freely from site to site. They delocalize across the neighboring sites which reduces the order parameter. After some time the electrons start dressing themselves with phonons thereby forming heavy polarons. The number of phonons in the system far exceeds the respective ground state phonon numbers plotted 


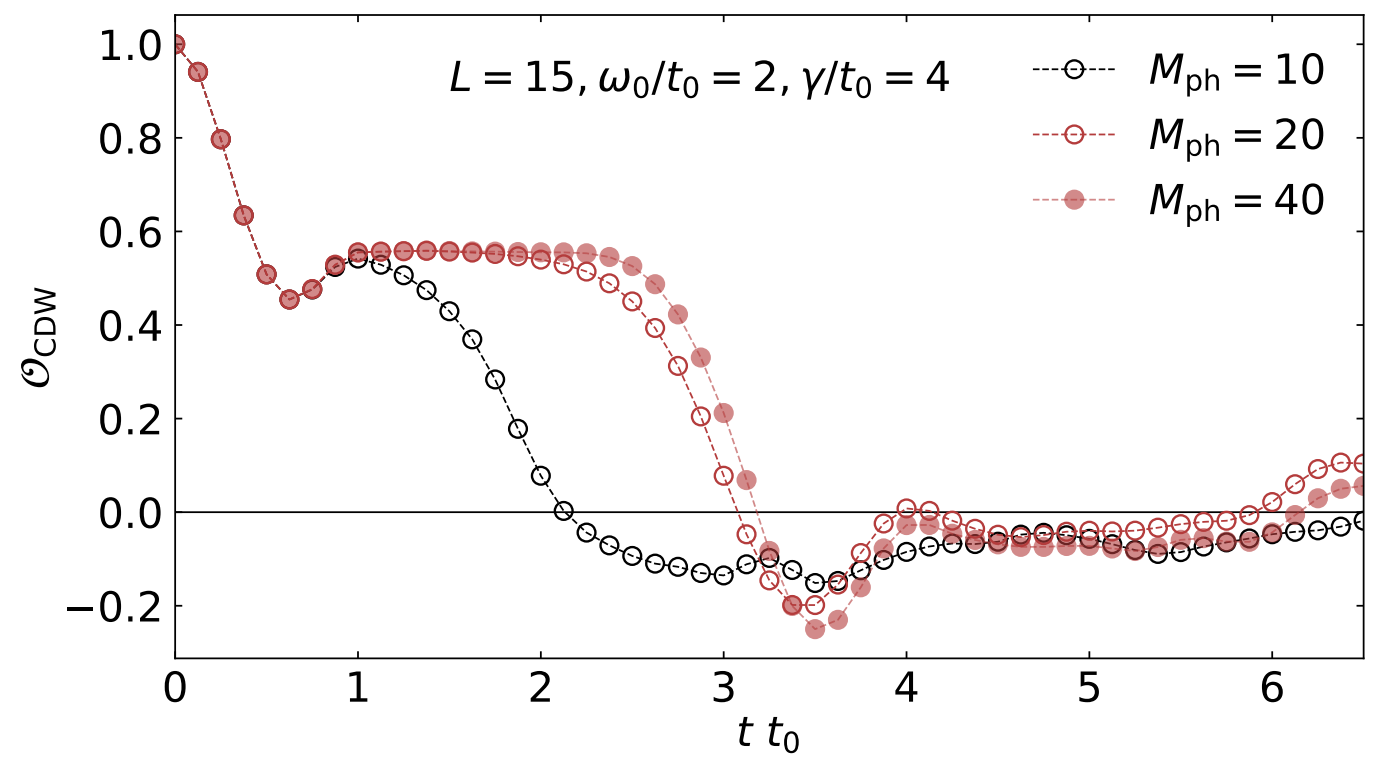

Figure 8.5: Bare CDW melting: order parameter $\mathcal{O}_{\mathrm{CDW}}$ as a function of time. $L=15, N=7, \omega_{0} / t_{0}=2$ and $\gamma / t_{0}=4 . M_{\mathrm{ph}}=10,20,40$. For clarity, only every fifth data point computed with TEBD-LBO is shown.

as dashed lines in Fig. 8.3(b). In case of the large couplings $\gamma / t_{0}=3$ and 4 the increased effective mass of the electrons resulting from the dressing with phonons impedes their movement so much that a plateau can be seen in the order parameter.

However, the electrons reabsorb the phonons after some time thus accelerating their movement again. The period of the oscillations in the phonon number is related to phonon frequency as $2 \pi / \omega_{0}$. This phenomenon can be seen as a temporal self trapping of the electrons [54].

In Fig. 8.4, the melting of the bare CDW state is shown for a larger phonon frequency of $\omega_{0} / t_{0}=10$. The increased frequency leads to several shorter plateaus in the decay of $\mathcal{O}_{\mathrm{CDW}}$ in case of the stronger coupling strengths $\gamma / t_{0}=15,20$ [Fig. 8.4(a)]. As in the case of $\omega_{0} / t_{0}=2$, the length of the plateaus in the order parameter are locked to the oscillations in the number of phonons [Fig. 8.4(b)]. For the weaker coupling strength $\gamma / t_{0}=5$, the melting is again comparable with the $\gamma=0$ case but with damped oscillations. These slow oscillations are superimposed by faster oscillations with the period of the oscillations in the phonon number.

To illustrate the convergence of the TEBD-LBO method in the local phonon cutoff, Fig. 8.5 shows the bare CDW melting for $L=15, \omega_{0} / t_{0}=2$, and $\gamma / t_{0}=4$ simulated with three different phonon cutoffs $M_{\mathrm{ph}}=10,20,40$. 


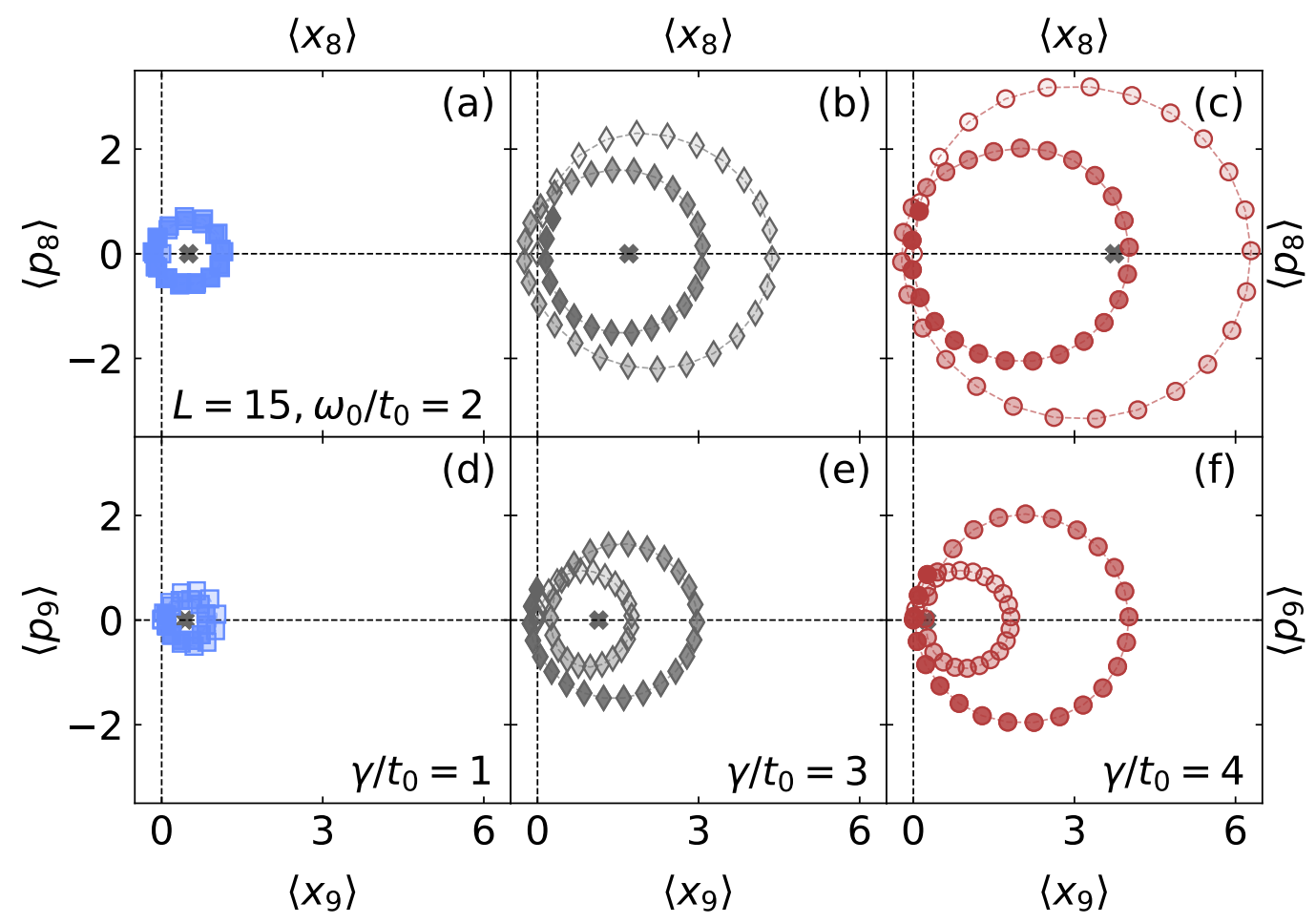

Figure 8.6: Bare CDW melting: phase-space dynamics of the local harmonic oscillators at sites $l=8,9$ that are initially occupied and not occupied, respectively. The time evolution can be followed by the shade of the symbols filling, where no filling corresponds to $t=0$ and completely filled corresponds to $t t_{0}=6.5 . L=15, N=7, \omega_{0} / t_{0}=2$ and $\gamma / t_{0}=1,3,4 . M_{\mathrm{ph}}=15,30,40$.

It is evident that the simulation with $M_{\mathrm{ph}}=10$ is not able to capture the first plateau in the order parameter and fails for $t>1 / t_{0}$. By doubling the cutoff to $M_{\mathrm{ph}}=20$ the results can be improved, but the plateau is still shorter than in the converged results with $M_{\mathrm{ph}}=40$. Note that the simulation with $M_{\mathrm{ph}}=40$ uses only up to $d_{\mathrm{o}}=12$ optimal modes. This comparison demonstrates the capabilities of the TEBD-LBO method to produce converged results in parameter regimes where a large phonon cutoff is necessary.

The temporal self trapping can be further understood by considering the movement of the local harmonic oscillators in phase space. In Fig. 8.6 the expectation value of the momentum:

$$
p_{l}=\mathrm{i}\left(b_{l}^{\dagger}-b_{l}\right)
$$


is plotted versus the expectation value of the displacement

$$
x_{l}=b_{l}^{\dagger}+b_{l}
$$

of the local oscillators. The time evolution can be followed by the shade of the symbols filling, where no filling corresponds to $t=0$ and completely filled corresponds to $t t_{0}=6.5$. Figs. 8.6(a),(b), and (c) correspond to site 8 in the system where there is an electron in the initial state while Figs. 8.6(d),(e), and (f) correspond to site 9 which is initially empty. The black cross marks the phase space position of the oscillators in the corresponding ground states.

In the initial state, the expectation values of $x_{l}$ and $p_{l}$ of all oscillators are zero since the initial state is an eigenstate of $H_{\mathrm{ph}}$, Eq. (3.1). As time evolves, the expectation values move through phase space. At $\gamma / t_{0}=1$ [Figs. 8.6(a) and (d)] the trajectory of the expectation values in phase space revolves around the ground-state expectation value. The behaviour is similar to a free harmonic oscillator initially displaced from its rest position.

For the larger couplings $\gamma / t_{0}=3$ [Figs. 8.6(b) and (e)] and $\gamma / t_{0}=4$ [Figs. 8.6(c) and (f)] the initial behavior of the oscillators is similar to the one at $\gamma / t_{0}=1$. However, the coupling to the electrons is apparent here since the amplitude of the oscillations are damped as the time evolves. From the phase space perspective, the self trapping happens if there is a mismatch of the displacement on neighboring sites. The displacements shift the energy of the local electronic states due to $H_{\text {el-ph }}$, Eq. (3.3). Therefore, a mismatch between neighboring sites means that the electron has to loose or gain energy when moving from one site to the next. This mechanism can strongly suppress the electron movement.

\subsection{Dressed charge-density-wave melting}

The second initial state considered here is the ground state of the Holstein Hamiltonian Eq. (3.4) in the atomic limit $t_{0}=0$. As discussed in Sec. 3.1 it has the form Eq. (3.12) where some of the sites are occupied by a small polaron while the rest of the sites are completely empty. The distribution of the polarons is completely arbitrary and every such state is a degenerate ground state at $t_{0}=0$. Here, the distribution is chosen such that the CDW order parameter Eq. 3.19 takes the value 1 in the initial state such that it takes the form:

$$
|\mathrm{DCDW}\rangle=\mathrm{e}^{-\frac{(L-1) \gamma^{2}}{4 \omega_{0}^{2}}}\left[\prod_{l=1}^{(L-1) / 2} c_{2 l}^{\dagger} \mathrm{e}^{\frac{\gamma}{\omega_{0}} b_{2 l}^{\dagger}}\right]|\emptyset\rangle_{\mathrm{el}}|\emptyset\rangle_{\mathrm{ph}},
$$


which will be referred to as dressed CDW state (DCDW). The initial state is prepared by constructing the bare CDW state and then performing an imaginary time evolution according to the Holstein Hamiltonian with $t_{0}=0$ and $\gamma / \omega_{0}$ set to the same value as in the later real time evolution. The apparent difference of the dressed CDW state compared to the bare CDW state Eq. (8.1) is that the electrons here are dressed with phonons already in the initial state while such a dressing has to appear dynamically in case of the bare CDW state.

The time evolution of the order parameter $\mathcal{O}_{\mathrm{CDW}}$ when starting from the dressed CDW state is plotted in Fig. 8.7(a). For smallest coupling $\gamma / t_{0}=1$ it can again be seen as a damped version of the case without coupling to the phonons plotted as black dots in Fig. 8.7(a). The order parameter tends to zero with oscillations around that value whose frequency is mainly controlled by the hopping matrix $t_{0}$. In contrast, for the larges coupling $\gamma / t_{0}=4$, the order parameter changes only very little over the course of the simulation time because the initial state is very close to the ground state in this case. For the intermediate coupling $\gamma / t_{0}=3$ the behavior interpolates between the small and large coupling behavior as $\mathcal{O}_{\mathrm{CDW}}$ tends to zero slowly without large fluctuations.

The number of phonons in the system over time is plotted in Fig. 8.7(b). In the initial states, the number of phonons is relatively close to the respective ground states for all couplings. In case of the strongest couplings the number of phonons in the system does not change considerably during the time evolution. This reflects the proximity of the initial state to the ground state. Different from that, the phonon number increases by a factor of two or three in the beginning of the simulation for $\gamma / t_{0}=1$. In this case the initial state is not that close to the ground state where polarons are delocalized over the whole lattice. The behavior of the phonon number at $\gamma / t_{0}=3$ is close to the one at $\gamma / t_{0}=4$ as it stays more or less stable over the course of the simulation time with small fluctuations.

In Fig. 8.8 the time evolution of the order parameter is plotted again but in contrast to Fig. 8.7(a) the time axis is in units of the effective hopping matrix element $\tilde{t}_{0}$, Eq. 3.15 , obtained in the small $t_{0}$ perturbation theory. The rescaling of the time axis reveals that the different timescales in Fig. 8.7(a) can be understood as stemming from the different effective masses of the polarons. As the coupling strength $\gamma / t_{0}$ is increased, the polarons become heavier and move slower through the lattice. Of course, the rescaling of the time axis does not yield a perfect collapse which is expected since the parameter sets used here are far away from the small $t_{0}$ limit.

One feature that stands out in Fig. 8.7(a) are peaks around $t t_{0} \approx 3.1$ and around $t t_{0} \approx 6.1$ for $\gamma / t_{0}=4$. Similar peaks can be found for $\gamma / t_{0}=3$. 


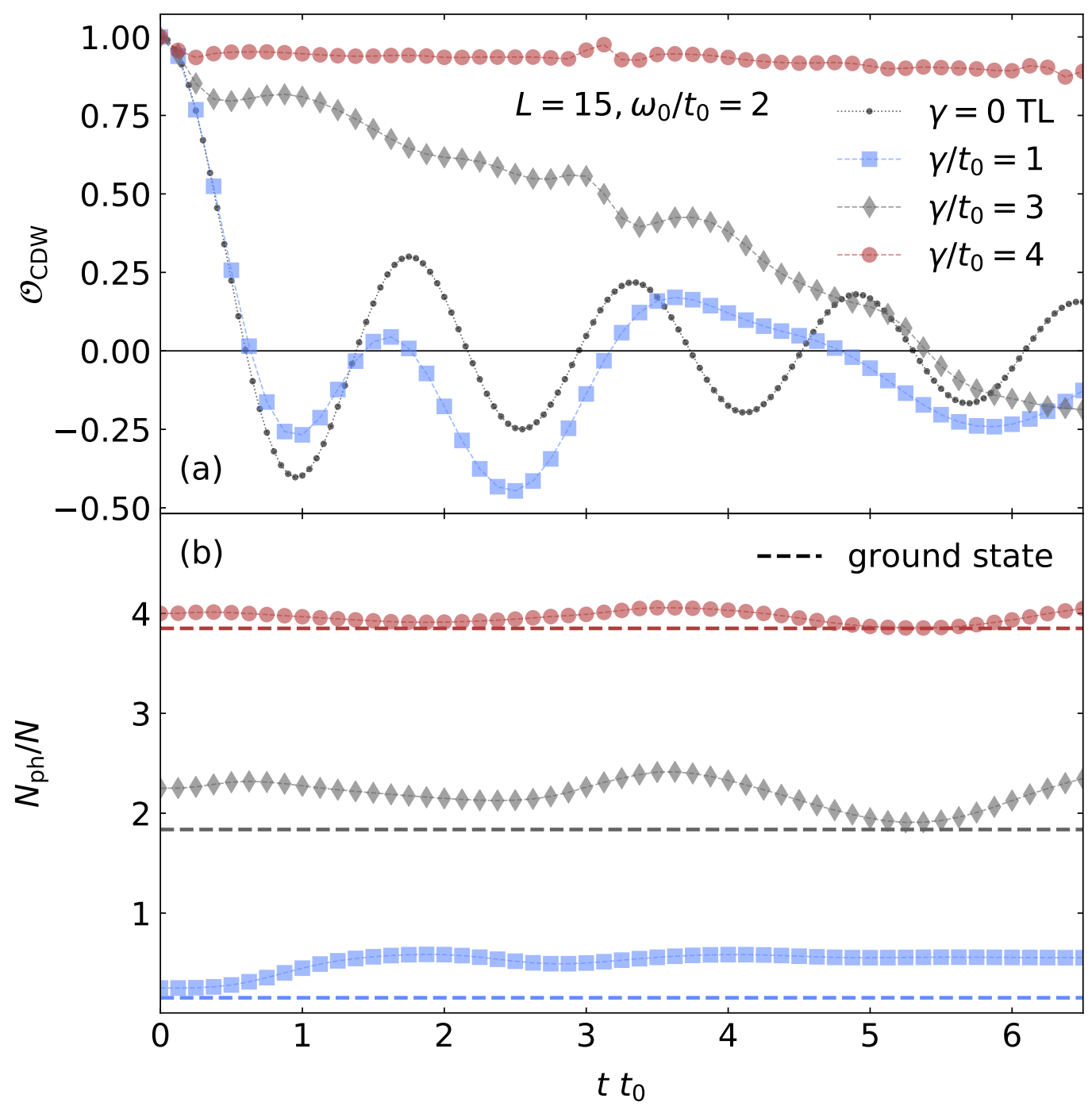

Figure 8.7: Dressed CDW melting: (a) order parameter $\mathcal{O}_{\mathrm{CDW}}$ (b) number of phonons in the system $N_{\mathrm{ph}} / N$ as a function of time. $L=15, N=7$, $\omega_{0} / t_{0}=2$ and $\gamma / t_{0}=1,3,4 . M_{\mathrm{ph}}=15,30,40$. For clarity, only every fifth data point computed with TEBD-LBO is shown. The small black dots in (a) correspond to exact analytical results for $\gamma=0$ in the thermodynamic limit when starting from the BCDW state [141]. The dashed lines in (b) mark the phonon numbers in the ground state at the respective parameters.

The positions of the peaks in time are roughly multiples of $2 \pi / \omega_{0}$. This relation can be checked by running similar simulations with different $\omega_{0} / t_{0}$ (not shown here). Despite this connection, the precise origin of the peaks remains unclear. 


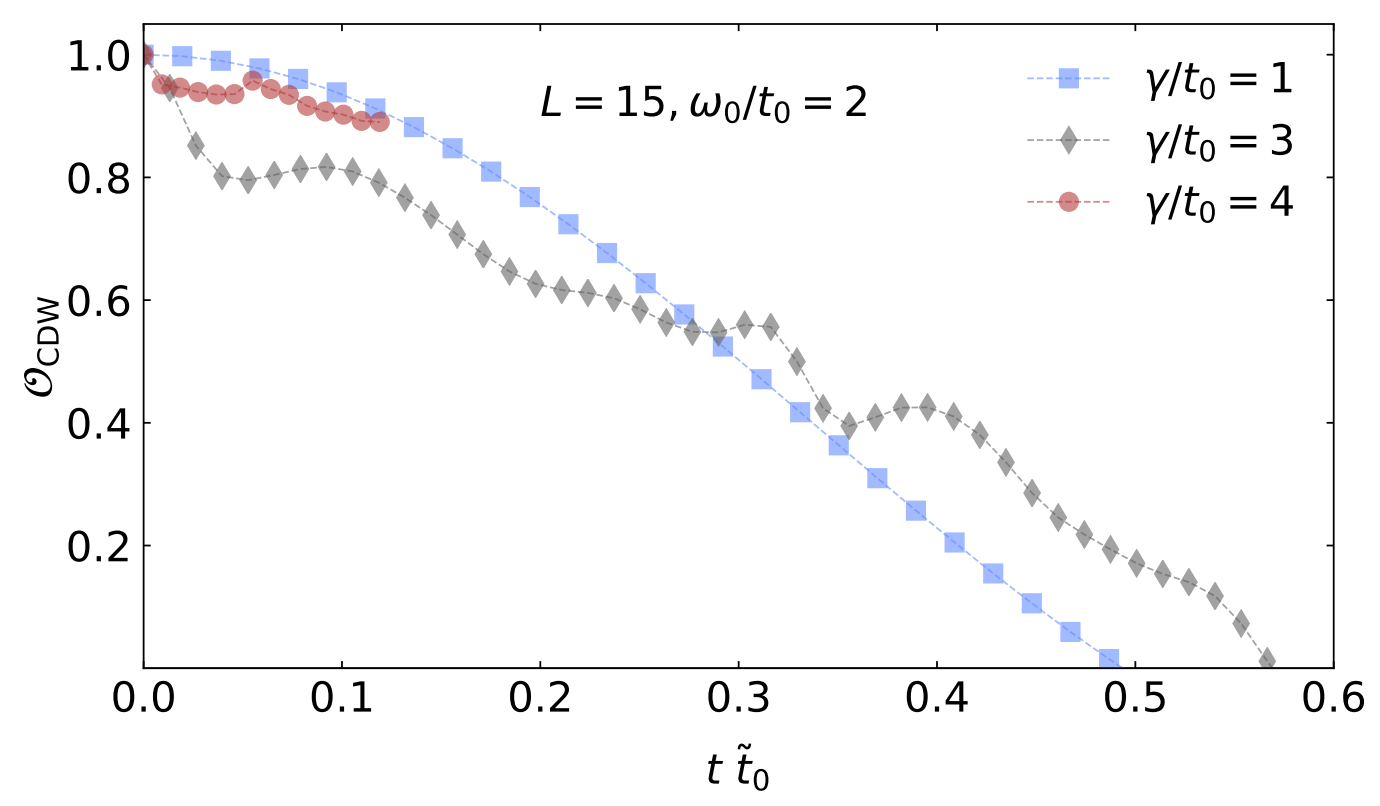

Figure 8.8: Dressed CDW melting: order parameter as function of time in units of the effective hopping matrix element $\tilde{t}_{0}$ Eq. 3.15. $L=15, N=7$, $\omega_{0} / t_{0}=2$ and $\gamma / t_{0}=1,3,4 . M_{\mathrm{ph}}=15,30,40$. For clarity, only every fifth data point computed with TEBD-LBO is shown for $\gamma / t_{0}=3$ and every twentieth data point is shown for $\gamma / t_{0}=4$.

The movement of the harmonic oscillators on sites 8 and 9 are plotted in Fig. 8.9 for $\omega_{0} / t_{0}=2, \gamma / t_{0}=1$ in panels (a) and (b), for $\gamma / t_{0}=3$ in panels (c) and (d) and for $\gamma / t_{0}=4$ in panels (e) and (f). Note that in this case the displacement in the initial state depends on weather an electron is on the site or not. The panels (a), (c), and (e) correspond to sites that are initially occupied by a fermion and therefore the expectation values of the displacements at $t t_{0}=0$ is nonzero. The panels (b), (d), and (f), however, correspond to sites that are initially empty such that the expectation values of the displacements are zero in the initial state. This is in contrast to the bare CDW state where the expectation values of the displacements on all sites are zero irrespective of whether there is a fermion on the site or not (cf. Fig. 8.6).

Note also the different ranges of values on the $x$ and $y$-axes for the different coupling strengths. This is done to reveal more detail in the phase space movement and reflects the very different behavior for the different coupling strengths.

As is the case for the evolution of the order parameter, the phase space movement for the weakest coupling $\gamma / t_{0}=1$ is comparable when starting 

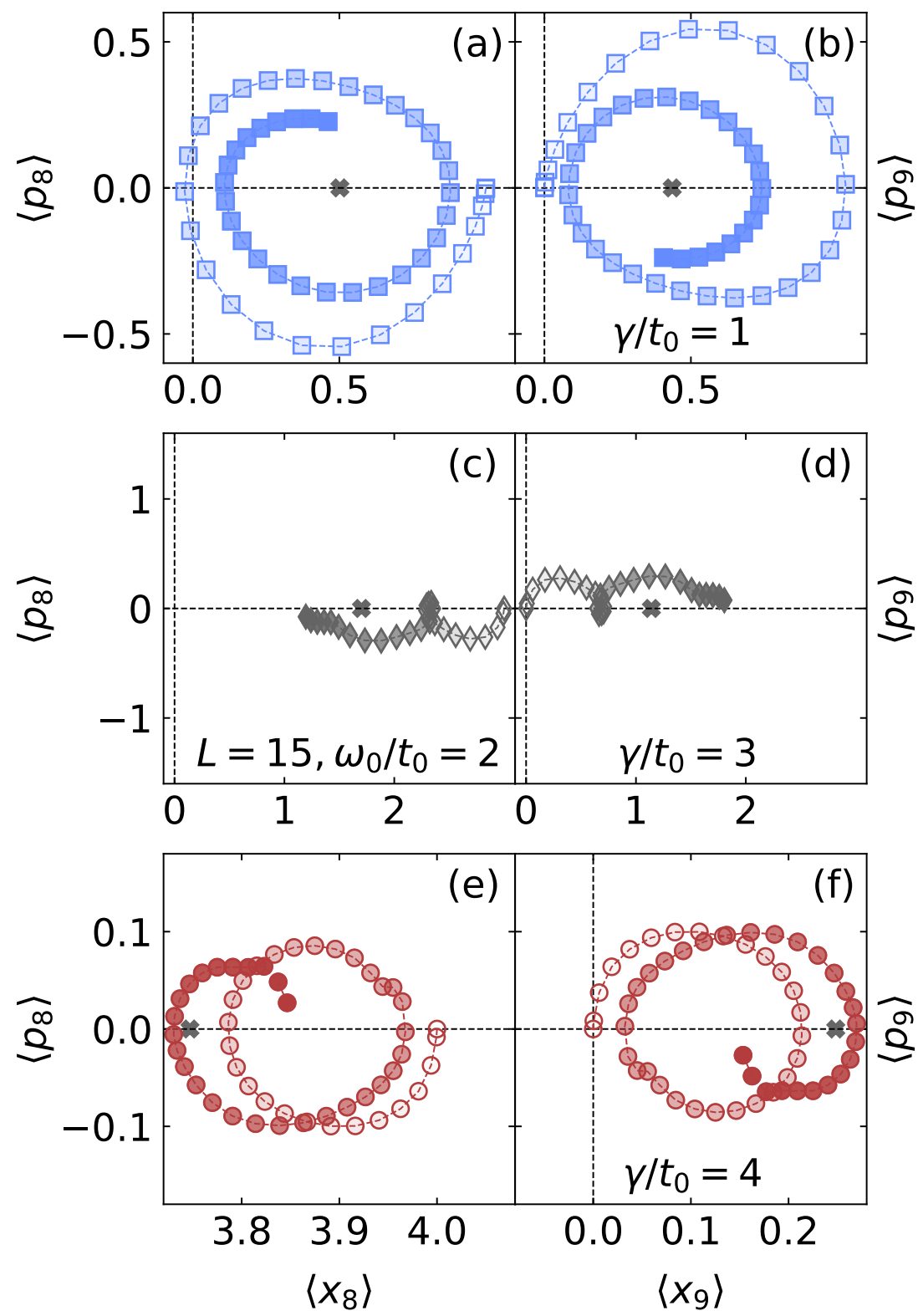

Figure 8.9: Dressed CDW melting: phase-space dynamics of the local harmonic oscillators at sites $l=8,9$ that are initially occupied and not occupied, respectively. The time evolution can be followed by the shade of the symbols filling, where no filling corresponds to $t=0$ and completely filled corresponds to $t t_{0}=6.5 . L=15, N=7, \omega_{0} / t_{0}=2$ and $\gamma / t_{0}=1,3,4 . M_{\mathrm{ph}}=15,30,40$.

from the bare CDW state [cf. Fig. 8.6(a) and (d)] and when starting from the dressed CDW state. The trajectory orbits the expectation value in the 
Table 8.1: Energy difference between the ground states and the initial states $\Delta E_{\mathrm{BCDW}[\mathrm{DCDW}]}=E_{\mathrm{BCDW}[\mathrm{DCDW}]}-E^{\mathrm{gs}}$ for the BCDW [DCDW] state with $L=15$ and $\omega_{0} / t_{0}=2$.

\begin{tabular}{ccc}
\hline \hline$\gamma / t_{0}$ & $\Delta E_{\mathrm{BCDW}} /\left(t_{0} N\right)$ & $\Delta E_{\mathrm{DCDW}} /\left(t_{0} N\right)$ \\
\hline 1 & 1.670 & 1.170 \\
3 & 4.877 & 0.377 \\
4 & 8.153 & 0.153 \\
\hline \hline
\end{tabular}

ground state, however, it spirals inwards for later times.

The behavior is very different for the intermediate coupling $\gamma / t_{0}=3$ [Fig. 8.9(c) and (d)]. The trajectories do not resemble persistent oscillations at all. Rather, the movement can be compared to the movement in the presence of friction. The trajectory moves towards the ground state displacement and even overshoots it but the momentum is always comparably small.

Yet another behavior can be observed for the largest coupling $\gamma / t_{0}=4$ [Fig. 8.9(e) and (f)]. Here the trajectories resemble circles again but the midpoint of the circle moves along the $x$-axis towards to displacement in the ground state.

\section{Comparison between bare CDW and dressed CDW melting}

In conclusion, there are large similarities between in the charge density wave melting when starting from the bare CDW state and the dressed CDW state in case of the small coupling $\gamma / t_{0}=1$. A fast decay of the order parameter $\mathcal{O}_{\mathrm{CDW}}$ to zero can be observed and the frequency of the oscillations in the order parameter are controlled by the hopping matrix element $t_{0}$ in both cases. The behavior for the larger couplings $\gamma / t_{0}=3,4$, on the other hand, is very different for the two initial states. The time evolution of the bare CDW state shows plateaus in the CDW order parameter that can be associated to a temporal self trapping of the polarons. The electrons undergo an oscillation between a bare electron without phonon dressing and a heavy polaron with a large phonon dressing and strongly modified effective mass. In case of the dressed CDW state as the initial state, the electrons already have a large phonon dressing from the start. This initial dressing gets stronger the larger the coupling $\gamma / t_{0}$ is. Therefore, the dynamics gets slowed down as the coupling strength is increased. Both of these strong coupling behaviors were similarly found by Kloss et al. [58] in the dynamics of single localized charge carriers placed in an otherwise empty Holstein lattice.

A further understanding of the dynamics can be gained by considering the energetic distance of the initial states from the respective ground states. 
In Table 8.1 these energy differences are given for the six considered cases. It is evident that the bare CDW states distance to the ground state grows as the coupling increases in contrast to the dressed CDW state that closes in on the ground state as $\gamma / t_{0}$ is increased. Energetic proximity of the initial state to the ground state means that few states in the many-body spectrum are available in the time evolution. This can explain the slow dynamics in case of the dressed CDW state at large $\gamma / t_{0}$.

\subsection{Quenches from the CDW to the metallic phase}

In the last two sections, the decay of CDW order was studied by considering initial product states with perfect CDW order. In this section, the initial state is a many-body ground state in the CDW phase with all its correlations. At $t=0$ the parameters of the Hamiltonian are quenched into the metallic TLL phase to start the dynamics.

The arrows in the phase diagram sketch Fig. 8.2 illustrate the two different quenches that are considered. The initial state for both of the quenches is the ground state at $\omega_{0} / t_{0}=2$ and $\gamma / t_{0}=4$. In the frequency quench (horizontal arrow in Fig. 8.2, FQ), the phonon frequency is quenched to $\omega_{0} / t_{0}=0.1$ and the coupling is quenched to $\gamma / t_{0}=0.2$, such that the ratio $\gamma / \omega_{0}$ stays constant before and after the quench. In the coupling quench (vertical arrow in Fig. 8.2, CQ), only the coupling is quenched to $\gamma / t_{0}=1$ while the phonon frequency remains unchanged.

To further understand the nature of the two quenches it is instructive to compare the quench energies $\Delta E^{\mathrm{qu}}=E^{\text {init }}-E^{\mathrm{gs}}$ as well as their kinetic $\Delta E_{\mathrm{kin}}^{\mathrm{qu}}=E_{\mathrm{kin}}^{\mathrm{init}}-E_{\mathrm{kin}}^{\mathrm{gs}}$ and phonon parts $\Delta E_{\mathrm{ph}}^{\mathrm{qu}}=E_{\mathrm{ph}}^{\mathrm{init}}-E_{\mathrm{ph}}^{\mathrm{gs}}$. The quench energies are the difference between the energy of the initial state after the quench $E^{\text {init }}$ and the ground state at the respective parameters $E^{\text {gs }}$, where $E_{\alpha}=\left\langle H_{\alpha}\right\rangle$ [cf. Eqs. (3.1) and (3.2)]. These energies are listed in Table 8.2.

There is a large difference between the total quench energies. This is due to the large difference in the phonon quench energy while the kinetic part

Table 8.2: Total quench energies $\Delta E^{\mathrm{qu}} /\left(t_{0} N\right)$ and the contributionsfrom the kinetic part $\Delta E_{\text {kin }}^{\mathrm{qu}} /\left(t_{0} N\right)$ and the phononic part $\Delta E_{\mathrm{ph}}^{\mathrm{qu}} /\left(t_{0} N\right)$.

\begin{tabular}{cccc}
\hline \hline & $\Delta E^{\mathrm{qu}} /\left(t_{0} N\right)$ & $\Delta E_{\mathrm{kin}}^{\mathrm{qu}} /\left(t_{0} N\right)$ & $\Delta E_{\mathrm{ph}}^{\mathrm{qu}} /\left(t_{0} N\right)$ \\
\hline $\mathrm{FQ}$ & 0.819 & 1.002 & 0.195 \\
$\mathrm{CQ}$ & 5.187 & 0.948 & 7.398 \\
\hline \hline
\end{tabular}


of the quench energy is very similar. In the frequency quench the phonon frequency is reduced in comparison to the electron bandwidth which keeps $\Delta E_{\mathrm{ph}}^{\mathrm{qu}}$ small although there is a phonon excess. In contrast, the phonon frequency remains unchanged with respect to the electron bandwidth in the coupling quench. The reduction of the coupling $\gamma / t_{0}$ leads to a phonon excess such that $\Delta E_{\mathrm{ph}}^{\mathrm{qu}}$ predominates in $\Delta E^{\mathrm{qu}}$.

\section{Frequency quench - FQ}

In Fig. 8.10(a) the CDW order parameter $\mathcal{O}_{\mathrm{CDW}}$ is plotted as a function of time after the quench. After the frequency quench the order parameter decays towards zero with oscillations around a value slightly larger than zero. The frequency of the oscillations is again controlled by the hopping $t_{0}$ as the comparison to the BCDW melting without phonon coupling reveals that is plotted as black dots in Fig. 8.10(a).

To understand this behavior the staggered displacement $\mathcal{O}_{\text {disp }}$ Eq. (3.20) is plotted in Fig. 8.10(b). As mentioned earlier a discrepancy between the displacements on neighboring sites leads to a mismatch between the electronic energy levels on these sites. A positive value of the staggered displacement means that it is energetically favorable for the electrons to sit on all the even sites, while a negative $\mathcal{O}_{\text {disp }}$ means that on the odd sites the electron levels are lower. As one can see in Fig. 8.10(b), the staggered displacement does not change much over the course of the simulation time in case of the frequency quench and at the end $\mathcal{O}_{\text {disp }}$ is still at $\approx 2.8$. This behavior is due to the small phonon frequency after the quench. The lattice just did not have time to react to the quench yet, while the electrons move around quicker but in front of a potential background given by the staggered displacement.

The slow movement of the oscillators can also be seen in Figs. 8.11(a) and (c) where the phase space movement of the oscillators on sites 8 and 9 is plotted over the course of the simulation time. One can see how the oscillators start moving in circles around the ground-state displacement but the movement is very slow and at the end of the simulation the displacement has only changed slightly.

To sum up, we see here very different timescales of the electrons and the local operators after the quench. The electrons have already relaxed to an almost steady state over the course of the simulation time. This relaxation is however only with respect to the remaining background potential generated by the slow local oscillators. These move on far longer timescales and are thus still far away from any relaxation. Therefore, a full relaxation of the system happens on timescales beyond the reach of the current method. 


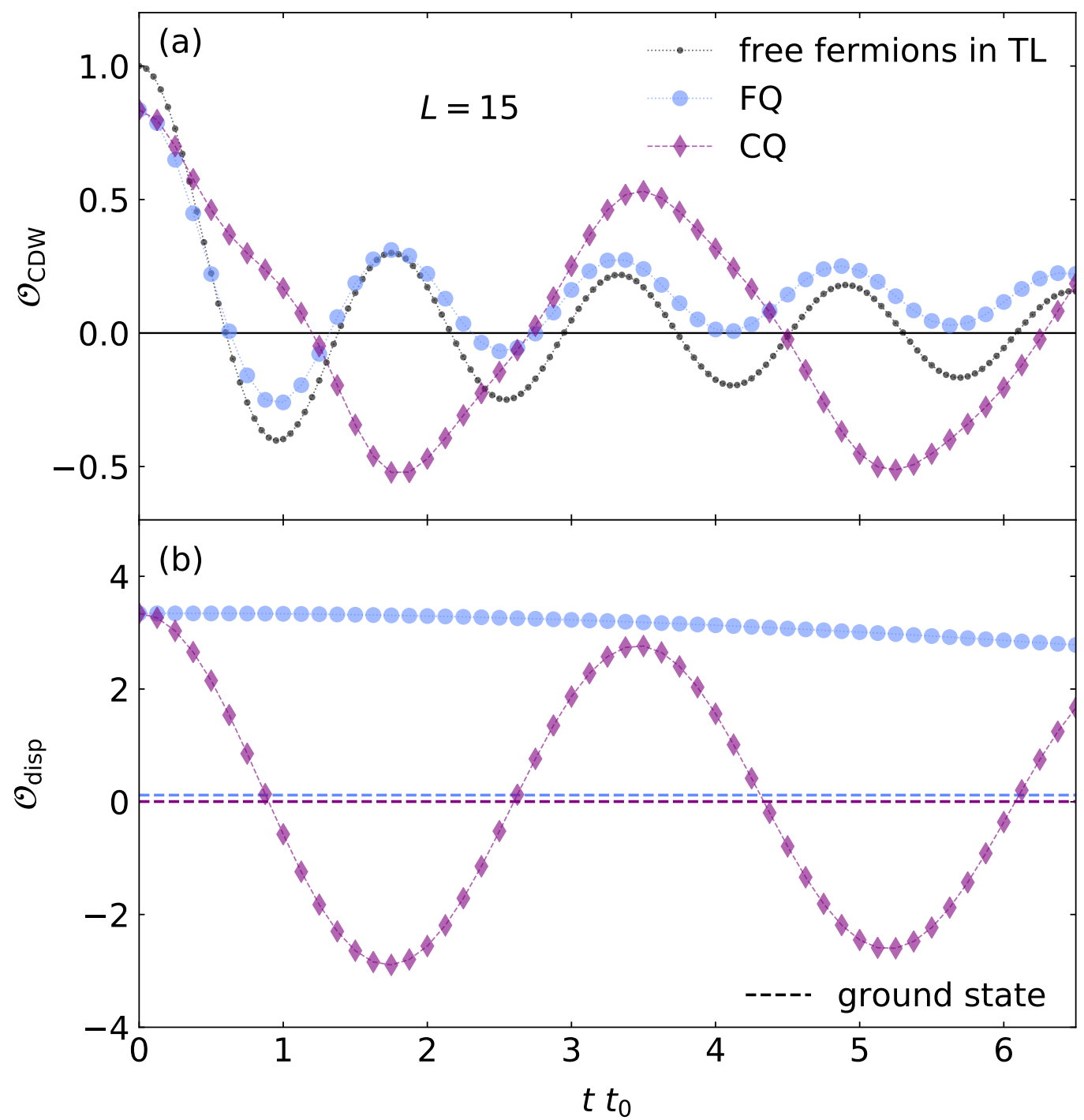

Figure 8.10: Quench from CDW phase to metallic phase: (a) electron order parameter $\mathcal{O}_{\mathrm{CDW}}$ (b) staggered displacement $\mathcal{O}_{\text {disp }}$ as a function of time. $L=15, N=7, M_{\mathrm{ph}}=40$. For clarity, only every fifth data point computed with TEBD-LBO is shown. The small black dots in (a) correspond to exact analytical results for $\gamma=0$ in the thermodynamic limit when starting from the BCDW state [141]. The dashed lines in (b) mark the staggered displacement $\mathcal{O}_{\text {disp }}$ in the ground state at the respective parameters.

\section{Coupling quench - CQ}

The dynamics after the coupling quench is very different. The CDW order parameter shows slow oscillations in Fig. 8.10(a) whose amplitude is almost 


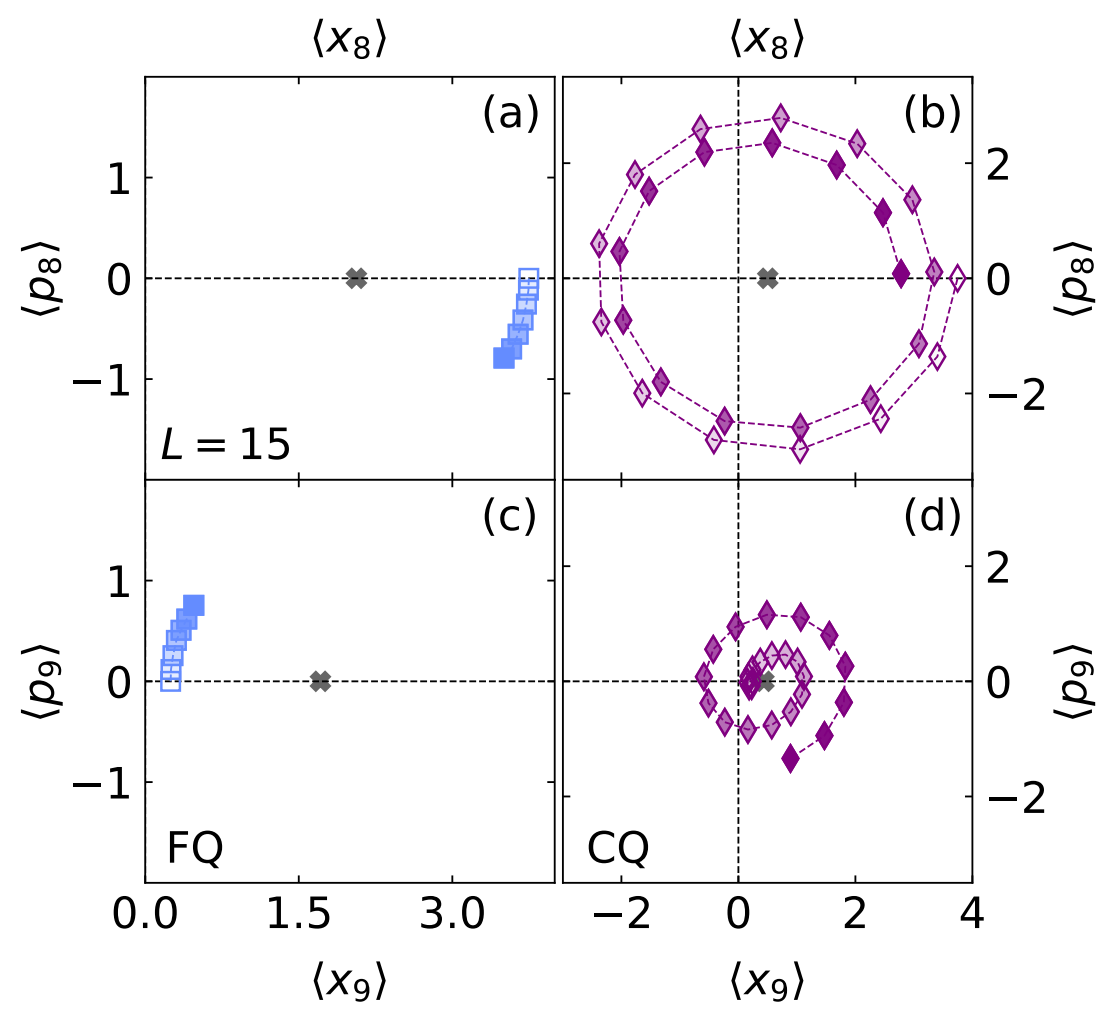

Figure 8.11: Quench from CDW phase to metallic phase: phase-space dynamics of the local harmonic oscillators at sites $l=8,9$ that are initially occupied and not occupied, respectively. The time evolution can be followed by the shade of the symbols filling, where no filling corresponds to $t=0$ and completely filled corresponds to $t t_{0}=6.5 . L=15, N=7, M_{\mathrm{ph}}=40$

constant. The oscillations in $\mathcal{O}_{\mathrm{CDW}}$ are simultaneous to the oscillations in the staggered displacement plotted in Fig. 8.10(b).

Again the dynamics can be understood by considering the displacement structure in the model as a background potential for the electrons. In this case the oscillations of the local oscillators are faster and change from positive to negative. When the sign of the $\mathcal{O}_{\text {disp }}$ changes from positive to negative the electrons are pushed away from the even sites to the odd sites. When the sign of the staggered displacement changes back to positive also the electrons are pushed back from the odd to the even sites.

The larger frequency of the local oscillators is evident in Fig. 8.11(b) and (d). The oscillator on site 8 performs two full oscillations during the simulation time with a slight damping. The oscillator on site 9 also starts moving but with smaller amplitude then the one on site 8 . Furthermore, there is a phase shift between the two oscillators of about $\pi / 2$ while the 
phase shift in case of the frequency quench is $\pi$.

\section{Comparison between frequency and coupling quench}

Comparing the two quenches, the transient dynamics studied here clearly depends on the initial state and the post quench parameters. The frequency quench can be understood by different timescales of the electron and oscillator movement. Electrons delocalize over the system quickly and the remaining order is due to the slower response of the phonon degrees of freedom to the quench. It is, however, interesting to see that the oscillators on both even and odd sites move by similar amounts in response to the faster moving electrons.

This is in contrast to the coupling quench where $O_{\mathrm{CDW}}$ and $O_{\text {disp }}$ are locked and in the beginning most of the oscillator movement is on the even sites that are initially occupied by electrons. The oscillators on the odd sites start moving significantly only after the electrons are pushed to the odd sites by the even site oscillators. These phenomena nicely demonstrate that transport in the Holstein model happens solely through motion of the electrons. Spatial inhomogeneities in the phonon distribution can only be dissolved through coupling to the electrons.

\subsection{Summary}

In this chapter, melting of CDW order was studied in the 1d Holstein model by starting from different initial states and simulating the real-time evolution with TEBD-LBO. In contrast to Ref. [57], the focus here was on the regime far from equilibrium and no direct interaction between electrons was present. Compared to completely free particles, overall the dynamics of charge carriers is slowed down. This is a common observation in Holstein-type systems, attributed to polaron formation resulting in an increased effective mass [57, $58,125,142]$ (see also [143]).

For weak electron-phonon coupling, the time evolution of the order parameter qualitatively follows the case of no coupling, with an additional damping. This behavior is found for both the bare and dressed CDW state. When $\gamma / t_{0}$ is larger, the behavior of $\mathcal{O}_{\mathrm{CDW}}$ in time differs drastically for the two initial states. In case the system is initialized in the bare CDW state, a transient self trapping effect occurs and plateaus can be observed in the time evolution of the order parameter. This phenomenon is not found when starting from the dressed CDW state. Here, the melting of the order parameter is just drastically slowed down as $\gamma / t_{0}$ is increased.

Quenching the system from the CDW to the TLL phase reveals different 
timescales for the relaxation of electron and phonon order. Phonons need to be absorbed and reemitted by electrons to move around the system and, therefore, inhomogeneity in the phonon sector can persist longer while the electronic order is mostly molten.

This study illustrates the capabilities of the TEBD-LBO method to account for a large number of local phonons in the time evolution while keeping the computational costs at a manageable level $[51,52]$. 


\section{Chapter 9}

\section{Thermalization in the Holstein model}

A related aspect to the short time dynamics after an excitation discussed in the previous chapter is the question weather and how closed quantum systems thermalize at later times. The unitary time evolution of quantum states ensures that a system, once prepared in a pure state and without contact to a heat bath will remain in a pure state. Thermalization can, therefore, only happen in the sense of subsystem thermalization, that is, parts of a system act as the bath for the complement of that part. The fundamental notion of how closed quantum systems thermalize is given by the eigenstate thermalization hypothesis (ETH) going back to the seminal works by Deutsch [59] and Srednicki [60]. In the following, I will review important aspects of the ETH, that become important in Sec. 9.2 where the ETH is tested in the Holstein polaron model [70]. The results presented here and in Ref. [70] are based on the bachelor and master thesis of D. Jansen. The author contributed to the production and independent verification of numerical data, as well as the analysis, discussion and writing of the paper.

\subsection{Eigenstate thermalization hypothesis}

Consider the unitary time evolution of a nonequilibrium state $|\psi\rangle$ under the Hamiltonian $H$ :

$$
|\psi(t)\rangle=\mathrm{e}^{-\mathrm{i} H t}|\psi\rangle=\sum_{n} \mathrm{e}^{-\mathrm{i} E_{n} t} \alpha_{n}|n\rangle
$$

where in the last step the state $|\psi\rangle$ was expanded into eigenstates $|n\rangle$ of the Hamiltonian $H$ with eigenenergies $E_{n}$ and coefficients $\alpha_{n}$. The question 
whether this state thermalizes during the time evolution can be reformulated as the question whether the time average of a local observable coincides with the thermal expectation value at late times [144]:

$$
\bar{O}=\lim _{t \rightarrow \infty} \frac{1}{t} \int_{0}^{t} \mathrm{~d} t^{\prime}\left\langle\psi\left(t^{\prime}\right)|O| \psi\left(t^{\prime}\right)\right\rangle \stackrel{?}{=}\langle O\rangle_{\mathrm{th}}
$$

where $\langle O\rangle_{\text {th }}$ is a thermal expectation value, for example the microcanonic one [144]:

$$
\langle O\rangle_{\mathrm{mc}, E}=\frac{1}{\Omega} \sum_{\left\{n: E_{n} \in[E, E+\Delta E]\right\}}\langle n|O| n\rangle .
$$

The sum runs over all eigenstates $|n\rangle$ of the considered Hamiltonian whose energy lies in a small energy window around the energy $E$ and $\Omega$ is the number of states within this window. For Eq. (9.2) to have any chance to hold, the energy $E$ has to be matched with the energy of the state $|\psi(t)\rangle$.

Using Eq. (9.1), the expectation value of the observable $O$ as a function of time is:

$$
\langle\psi(t)|O| \psi(t)\rangle=\sum_{\substack{n, m \\ n \neq m}} \mathrm{e}^{-\mathrm{i}\left(E_{m}-E_{n}\right) t} \alpha_{n}^{*} \alpha_{m}\langle n|O| m\rangle+\sum_{n}\left|\alpha_{n}\right|^{2}\langle n|O| n\rangle .
$$

Under the assumption that no degeneracies exist in the spectrum of $H$, the time average is equal to the second term of Eq. (9.4):

$$
\bar{O}=\sum_{n}\left|\alpha_{n}\right|^{2}\langle n|O| n\rangle
$$

Therefore, the time average depends on the exact initial state through all the coefficients $\alpha_{n}$ and on the expectation values of the observable $O$ in the eigenstates $|n\rangle$. It remains the question, under which condition this time average is equal the microcanonical average.

The ETH can give an answer to this question. It actually provides a set of conditions that guarantee the equality of the microcanonical average and the time average. It can even give a far stronger statement, namely that the expectation value of the operator $O$, Eq. (9.4), is almost always equal to the microcanonical expectation value, Eq. (9.3), for all times later than some thermalization time $[61,62]$.

\subsubsection{Quantum chaos}

The thermalization of classical systems is connected to their behavior being ergodic and chaotic [145]. To get a notion of how this carries over to the 
quantum case, one can study quantum systems which have a classical counterpart whose behavior is chaotic. It has been found that in certain systems of this type, the Hamiltonian of the quantum system shares properties with random matrices $[62,146]$. In particular, energy level repulsion appears and for time reversal symmetric problems the distribution of differences between consecutive levels $s$ follows a Wigner-Dyson distribution [62]:

$$
\mathcal{P}_{\mathrm{WD}}(s)=\frac{\pi s}{2} \mathrm{e}^{-\pi s^{2} / 4} .
$$

This distribution can be rigorously derived for $2 \times 2$ matrices form the Gaussian orthogonal ensemble (GOE) [62]. For larger matrices from the GOE no closed form for the level spacing distribution exist but the statistics are qualitatively and quantitatively close to Eq. (9.6) [62]. For quantum systems without a classical counterpart the property that the distribution of the energy level spacings follow a Wigner-Dyson distribution can be taken as a definition for quantum chaos [62].

\subsubsection{ETH ansatz}

Based on the assumption that thermalizing many-body Hamiltonians look like random matrices, Srednicki formulated the ETH ansatz for matrix elements of (local) observables in semiclassical systems [61]:

$$
\langle n|O| m\rangle=O(\bar{E}) \delta_{n m}+\mathrm{e}^{-S(\bar{E}) / 2} f_{O}(\bar{E}, \omega) R_{n m},
$$

where $\bar{E}=\left(E_{n}+E_{m}\right) / 2, \omega=E_{n}-E_{m}$, and $S(\bar{E})$ is the thermodynamic entropy at $\bar{E}$. The functions $O(\bar{E})$ and $f_{O}(\bar{E}, \omega)$ are required to be smooth. $R_{n m}$ are random numbers with mean zero and unit variance. This ansatz is now used in the test of thermalization in generic quantum systems (see eg. Refs. [62, 63, 65, 66, 70, 147-149]). The derivation of Eq. (9.7) is not rigorous but its form can be inferred from random matrix theory $[61,150]$. Especially the exact properties of the function $f_{O}(\bar{E}, \omega)$ are still discussed (see eg. Refs. [76, 151,152].

It is apparent that the ETH ansatz Eq. (9.7) guarantees thermalization according to Eq. (9.2). Under the assumption that the distribution of the coefficients $\alpha_{n}$ in Eq. (9.1) are peaked around a the energy expectation value of $|\psi\rangle$ the time average Eq. (9.5) will indeed yield the microcanonical average Eq. (9.3). The smooth function $O(\bar{E})$ will not vary much in the small energy window such that the exact distribution of the coefficients $\alpha_{n}$ does not affect the result of Eq. 9.5 as long as the distribution is narrow enough such that it fits in the microcanonical energy window of Eq. (9.3). 
The form of the offdiagonal matrix elements in the ETH ansatz further predicts the stronger form of thermalization mentioned above, that the expectation value of local observables for almost every point in time is the same as the microcanonical prediction [61,62]. The level spacings are random numbers drawn from a Wigner-Dyson distribution Eq. (9.6) which tells us that the offdiagonal part of Eq. (9.4) is subject to dephasing and therefore will average to zero. This is at least true if there are no single offdiagonal elements that are of the same order as the diagonal elements which is exactly what the ETH ansatz ensures.

A further implication of Eq. (9.7) is that every eigenstate from the bulk of the spectrum of a Hamiltonian that is quantum chaotic is already thermal. Since the function $O(\bar{E})$ is smooth it is enough to calculate the expectation value of $O$ in a single eigenstate that lies in the microcanonical window to predict the microcanonical expectation value Eq. (9.3).

So far no proper definition was given for which observables the ETH ansatz Eq. (9.7) is actually valid (see eg. Ref. [153] for a discussion of this problem). There are of course certain observables that certainly do not have the given structure. An important example are the projectors on eigenstates of the Hamiltonian $|n\rangle\langle n|$. The expectation values of the projectors are the absolute values squared of the coefficients $\alpha_{n}$ which are conserved quantities. These quantities certainly do not thermalize and, therefore, have to be excluded.

\subsubsection{Exceptions from ETH}

Importantly, there are exceptions from the thermalizing behavior discussed above. These include integrable models. In these models there exists an extensive number of conserved quantities that prevent them from thermalizing in the conventional manner. Furthermore, there is no level repulsion and therefore the distribution of the level spacings can be shown to follow a Poissonian shape [62]:

$$
\mathcal{P}_{\text {Poi }}(s)=\mathrm{e}^{-s}
$$

\subsection{ETH test in the Holstein polaron model}

In the this section the ETH will be tested in the Holstein model Eq. (3.4) (see also Ref. [70]). The number of electrons in the system will be restricted to one (Holstein polaron model). This restriction, however, makes this study particularly interesting since the coupling energy, which renders the model 
non integrable, is not extensive in this case. At first glance one could expect that such a perturbation of $O(1)$ does not affect the model in the thermodynamic limit and therefore the model should look less quantum chaotic the larger the system is. However, it is known that perturbations of $O(1)$ can in fact be enough to render a system quantum chaotic [71-76].

Since a full diagonalization of the Hamiltonian is necessary for the ETH study presented here the system size has to be restricted to relatively small clusters of up to $L=8$ sites. Further, no more than $M_{\mathrm{ph}}=3$ phonons per site can be accounted for. Periodic boundary conditions are used and by utilizing the translation invariance of the Hamiltonian the dimension of every $k$ sector of the Hilbert space is $\operatorname{dim}\left(\mathcal{H}^{\{k\}}\right)=\left(M_{\mathrm{ph}}+1\right)^{L}$ while the full Hilbert space has dimension $\mathcal{D}=\operatorname{dim}(\mathcal{H})=L\left(M_{\mathrm{ph}}+1\right)^{L}$. Therefore, largest matrices that have to be diagonalized have $\operatorname{dim}\left(\mathcal{H}^{\{k\}}\right)=65536$.

\subsubsection{Quantum chaos indicators}

The first task is to check whether the spectrum of the Holstein Hamiltonian follows the predictions from random-matrix theory. This is done as a function of the parameters in the Hamiltonian, the system size $L$, and the maximum number of phonons per site $M_{\mathrm{ph}}$ to see how a quantum chaotic behavior arises. The average energy of a state in the Holstein polaron model is [70]:

$$
E_{\mathrm{av}}=\frac{1}{\mathcal{D}} \operatorname{Tr}\left[H_{\mathrm{Hol}}\right]=\frac{L M_{\mathrm{ph}} \omega_{0}}{2}
$$

To choose an energy-density window around the center of the spectrum a parameter $\eta$ is introduced, such that

$$
\frac{E_{\mathrm{av}}-E_{\alpha}}{E_{\mathrm{av}}-E_{\mathrm{min}}}<\eta \quad \text { if } \quad E_{\alpha}<E_{\mathrm{av}}
$$

and

$$
\frac{E_{\alpha}-E_{\mathrm{av}}}{E_{\mathrm{max}}-E_{\mathrm{av}}}<\eta \quad \text { if } \quad E_{\alpha}>E_{\mathrm{av}}
$$

The set of eigenstates within the target energy-density window is then:

$$
\mathcal{Z}_{\eta}^{k}=\left\{|\alpha\rangle: E_{\alpha} / E_{\mathrm{av}} \in[1-\eta, 1+\eta]\right\}
$$

To study the statistical properties of the spectrum one has to restrict the states to a single symmetry sector $[62,154]$. Therefore, the following study of quantum chaos indicators will be restricted to the $k=2 \pi / L$ quasimomentum sector and $\eta=2 / 3$ is used. 
To analyse the statistics of the energy level spacings $\delta_{\alpha}=E_{\alpha+1}-E_{\alpha}$ an unfolding procedure is necessary $[155,156]$. The unfolding is done to normalize the mean level spacing to one and a comparison with the WignerDyson distribution Eq. (9.6) is possible. To circumvent the unfolding one can instead consider the dimensionless gap ratio:

$$
r_{\alpha}=\frac{\delta_{\alpha}}{\delta_{\alpha-1}}
$$

and define the restricted gap ratio from it [157]:

$$
\tilde{r}_{\alpha}=\frac{\min \left\{\delta_{\alpha}, \delta_{\alpha-1}\right\}}{\max \left\{\delta_{\alpha}, \delta_{\alpha-1}\right\}}=\min \left\{r_{\alpha}, r_{\alpha}^{-1}\right\}
$$

In case of a non chaotic system with Poisson statistics of the level spacings the distribution of the restricted gap ratio is [158]:

$$
\mathcal{P}_{\mathrm{Poi}}(\tilde{r})=\frac{2}{(1+\tilde{r})^{2}}
$$

with the average $\tilde{r}_{\mathrm{Poi}}=2 \ln 2-1 \approx 0.38629$ [158]. In case of matrices from the GOE a closed form can be derived for $3 \times 3$ matrices [158]:

$$
\mathcal{P}_{\mathrm{GOE}}(\tilde{r})=\frac{27}{4} \frac{\tilde{r}+\tilde{r}^{2}}{\left(1+\tilde{r}+\tilde{r}^{2}\right)^{5 / 2}}
$$

with average $\tilde{r}_{\mathrm{GOE}}=4-2 \sqrt{3} \approx 0.5359$ [158]. A slightly different result can be obtained by numerically diagonalizing $1000 \times 1000$ random matrices with from the GOE which yields $\tilde{r}_{\mathrm{GOE}, \mathrm{num}}=0.5307$ [158].

An example for the distribution of the restricted gap ratios in case of a quantum chaotic system is shown in Fig. 9.1(b). The blue line shows the histogram of the occurrence of $\tilde{r}_{\alpha}$ for a system with $L=8, M_{\mathrm{ph}}=3$, $\omega_{0} / t_{0}=1 / 2$, and $\gamma / t_{0}=1 / \sqrt{2}$. The histogram follows the prediction for random matrices from the GOE (solid black line) and is clearly distinct from the result for Poisson distributed level spacings shown as dashed line.

The analysis in Figs. 9.1(a), (c), and (d) focuses on the the average restricted gap ratio $\tilde{r}_{\mathrm{av}}=\langle\tilde{r}\rangle_{\eta}$ which is the average over the set $\mathcal{Z}_{\eta}^{k}$. The average gives a measure of the proximity of the distribution of energy gaps to a Poisson or Wigner-Dyson distribution. In Fig. 9.1(a), $\tilde{r}_{\mathrm{av}}$ is plotted against the coupling strength $\gamma / t_{0}$ at $\omega_{0} / t_{0}=1 / 2$ and for different system sizes $L=6,7,8$ with $M_{\mathrm{ph}}=3$. It is evident that $\tilde{r}_{\mathrm{av}}$ converges to the large GOE matrix prediction $\tilde{r}_{\mathrm{GOE}, \text { num }}=0.5307$ (solid line) in a wide range of $\gamma / t_{0}$ as the system size is increased. Clearly, the difference to the analytic result for $3 \times 3$ matrices $\tilde{r}_{\mathrm{GOE}} \approx 0.5359$ (dashed line) can be resolved. 

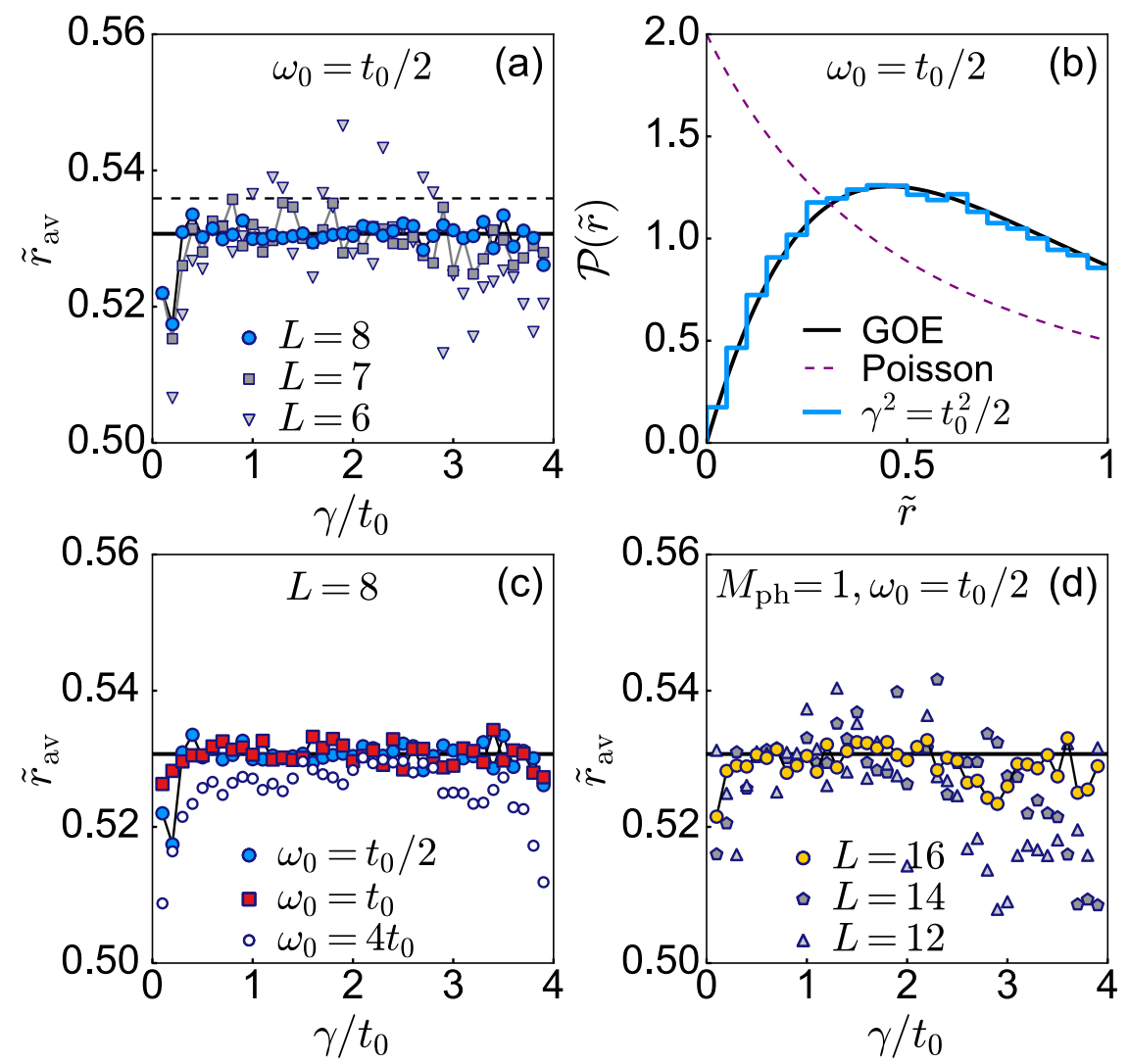

Figure 9.1: Average of the restricted gap ratio $\tilde{r}_{\mathrm{av}}$ as a function of $\gamma / t_{0}$ (a) for different system sizes at $\omega_{0} / t_{0}=1 / 2$ and $M_{\mathrm{ph}}=3$, (c) for different phonon frequencies $\omega_{0} / t_{0}$ at $L=8$ and $M_{\mathrm{ph}}=3$, and (d) for different system sizes at $\omega_{0} / t_{0}=1 / 2$ and $M_{\mathrm{ph}}=1$. The dashed line in (a) represents the GOE prediction for $3 \times 3$ matrices $\tilde{r}_{G O E} \approx 0.5359$ [158]. The solid lines in (a), (c), and (d) represent the numerical GOE result for $1000 \times 1000$ matrices $\tilde{r}_{\mathrm{GOE}, \mathrm{num}}=0.5307$ [158]. (b) Histogram of the restricted gap ratios $\tilde{r}$ for $L=8, M_{\mathrm{ph}}=3, \omega_{0} / t_{0}=1 / 2$, and $\gamma / t_{0}=1 / \sqrt{2}$. The dashed line shows the expectation from a Poisson statistics $\mathcal{P}_{\text {Poi }}(\tilde{r})$, while the solid line shows the prediction for $3 \times 3$ matrices from a $\operatorname{GOE} \mathcal{P}_{\mathrm{GOE}}(\tilde{r})$ [158]. Figure from Ref. [70].

For small $\gamma / t_{0}$ the proximity to the integrable point $\gamma=0$ becomes noticeable as $\tilde{r}_{\mathrm{av}}$ is reduced in this limit especially for small systems. This effect is reduced as the system size increases.

In Fig. 9.1(c), $\tilde{r}_{\mathrm{av}}$ is again plotted against $\gamma / t_{0}$ but this time for different phonon frequencies $\omega_{0} / t_{0}$ at a fixed system size of $L=8$ and $M_{\mathrm{ph}}=3$. The overall effect of increasing the phonon frequency is that the agreement with 
the GOE prediction gets worse. This is particularly evident for the largest couplings $\gamma / t_{0}$ studied here. In this case a connection to the atomic limit $t_{0}=0$ (large $\gamma / t_{0}$ and $\omega_{0} / t_{0}$ ) of the Holstein model can be made which is a second integrable limit (cf. Sec 3.1).

In Fig. 9.1(d) the influence of the phonon cutoff is analysed. Here, $M_{\mathrm{ph}}=$ $1, \omega_{0} / t_{0}=1 / 2$, and the system sizes are $L=12,14,16$. The system sizes are chosen such that the Hilbert space dimensions for the three datasets are the same as the ones in Fig. 9.1(a) for $L=6,7,8$. Although, the agreement with the GOE prediction is less good as in Fig. 9.1(a) for $M_{\mathrm{ph}}=3$ the fluctuations still decrease as the system size is increased.

The results from Fig. 9.1(a) and (d) bring confidence that the thermalization analysis done here allows for conclusions in the thermodynamic limit. A truly infinite system would of course mean that both the system length $L$ as well as the phonon number per site $M_{\mathrm{ph}}$ have to be taken to infinity and the question remains in which order the limits have to be taken. However, already the case of $M_{\mathrm{ph}}=1$ (hard core bosons) shows convincing agreement with GOE predictions. A systematic study of the limit $M_{\mathrm{ph}} \rightarrow \infty$ and its influence on quantum chaos indicators remains an open question. In the following, the analysis of the operators in the Holstein model will be restricted to $M_{\mathrm{ph}}=3$ and the influence of the phonon cutoff is neglected.

\subsubsection{Diagonal elements of observables}

To check the applicability of the ETH ansatz (9.7) to the Holstein model it has to be tested whether matrix elements of observables in the eigenbasis of the Hamiltonian follow the predictions in the thermodynamic limit. In this section the diagonal elements will be analysed while the following section is devoted to the offdiagonal matrix elements.

The analysis focuses on four different observables. Two of these observables measure properties of the fermion in the system, namely the fermion kinetic energy $H_{\text {kin }}$, Eq. (3.2), and the quasimomentum occupation:

$$
m_{q}=\frac{1}{L} \sum_{j, l=1}^{L} \mathrm{e}^{\mathrm{i}(l-j) q} c_{j}^{\dagger} c_{l}
$$

at $q=0$. The two observables are of course related, however, thermalization of $m_{q=0}$ does not immediately imply thermalization of $H_{\text {kin }}$. Both electronic observables are intensive quantities since there is only one electron present in the system.

The other two measure properties from the phonon sector, namely the average phonon number per site in the system $N_{\mathrm{ph}}=1 / L \sum_{l=1}^{L} b_{l}^{\dagger} b_{l}=H_{\mathrm{ph}} /\left(L \omega_{0}\right)$ 
and the nearest-neighbor offdiagonal matrix element of the phonon one-body correlation matrix:

$$
T_{1}=\frac{1}{L} \sum_{j}^{L}\left(b_{j}^{\dagger} b_{j+1}+b_{j+1}^{\dagger} b_{j}\right) .
$$

From the four observables there are two that are part of the Hamiltonian $\left(H_{\text {kin }}\right.$ and $\left.N_{\mathrm{ph}}\right)$, one from each sector, and two are not $\left(m_{q=0}\right.$ and $\left.T_{1}\right)$.

In Fig. 9.2(a)-(d) the diagonal matrix elements of the aforementioned observables $\langle\alpha|O| \alpha\rangle$ in the eigenbasis of the Hamiltonian (eigenstate expectation values) are plotted against the energy of the respective eigenstates $E_{\alpha} / E_{\mathrm{av}}$ in the $k=2 \pi / L$ sector. The parameters of the Hamiltonian are $\omega_{0} / t_{0}=1 / 2, \gamma / t_{0}=1 / \sqrt{2}, M_{\mathrm{ph}}=3$, and different system sizes $L=6$ (red dots), $L=7$ (dark blue dots), and $L=8$ (light blue dots). The dots for $L=6$ are plotted in the background, the dots for $L=7$ in the middle, and the dots for $L=8$ in the foreground. For all four observables one can observe that the distribution of the eigenstate expectation values becomes narrower in the bulk of the spectrum as the system size is increased (arrows in Fig. 9.2(a)). As expected the fluctuations at the edges of the spectrum are larger. These observations suggest that the eigenstate expectation values indeed become smooth functions of the energy in the thermodynamic limit as the ETH ansatz predicts.

To quantify this convergence of the distribution of the eigenstate expectation values towards a smooth function the eigenstate-to-eigenstate fluctuations of the expectation values can be analysed [65,159]:

$$
z_{\alpha}(O)=\langle\alpha+1|O| \alpha+1\rangle-\langle\alpha|O| \alpha\rangle \text {. }
$$

In Figs. 9.3(a)-(d) two different quantities are analysed. The average fluctuation defined as:

$$
\langle z\rangle_{\eta}(O)=\frac{1}{\left\|\mathcal{Z}_{\eta}\right\|} \sum_{|\alpha\rangle \in \mathcal{Z}_{\eta}}\left|z_{\alpha}(O)\right|,
$$

where $\left\|\mathcal{Z}_{\eta}\right\|$ is the number of states in the set $\mathcal{Z}_{\eta}$, and the maximal fluctuation:

$$
z_{\max }(O)=\max _{|\alpha\rangle \in \mathcal{Z}_{\eta}}\left|z_{\alpha}(O)\right|
$$

The energy density window fixed by the parameter $\eta$ means that the set $\mathcal{Z}_{\eta}$ includes an increasing fraction of all states in as the system size is increased. In fact, this fraction will approach 1 as $L \rightarrow \infty$ and for $L=8$ 

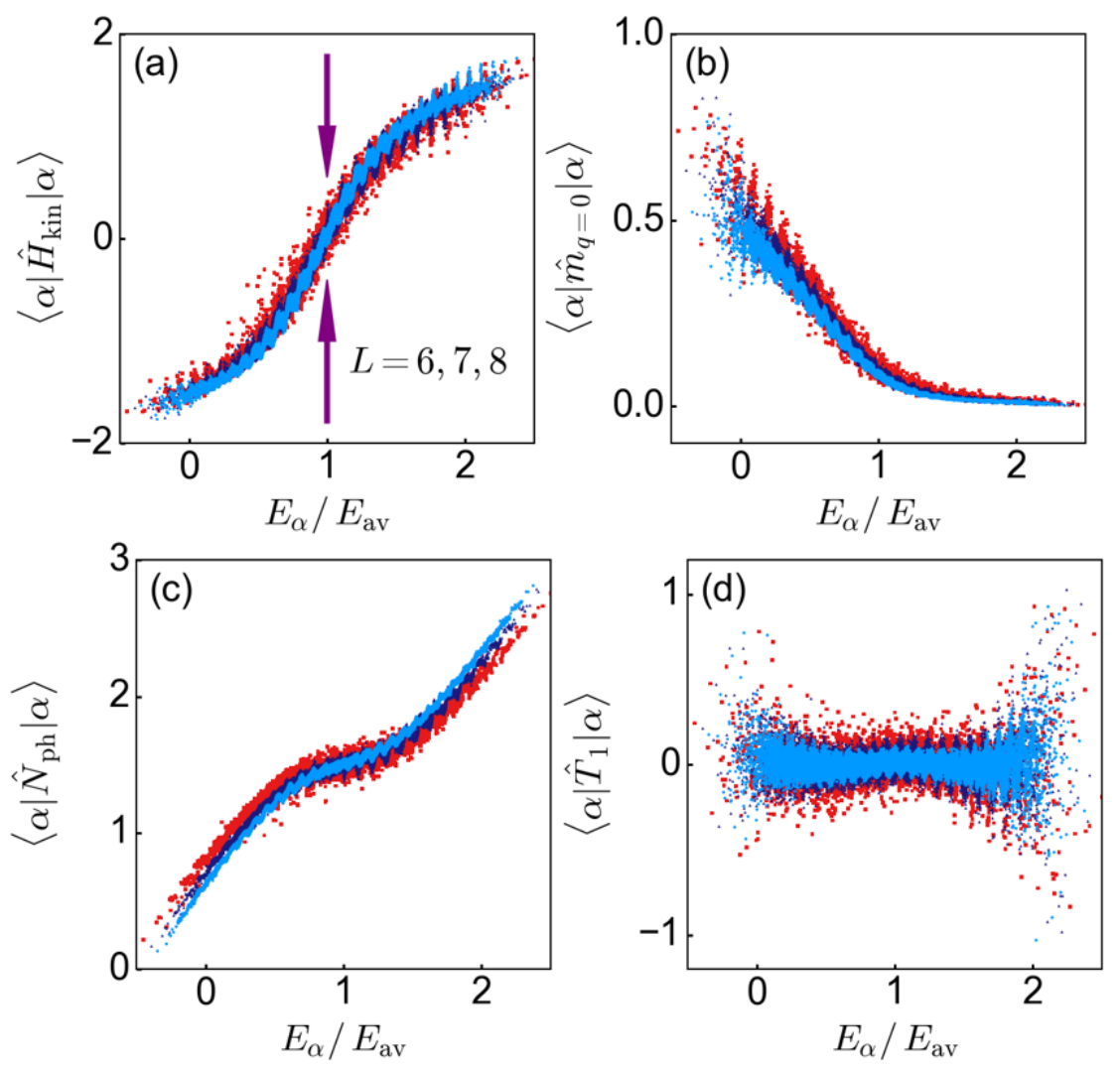

Figure 9.2: Diagonal matrix elements in the Hamiltonian eigenbasis of (a) the fermion kinetic energy $H_{\text {kin }}$, (b) the quasimomentum occupation at $q=0$, $m_{q=0}$, (c) the average phonon number in the system $N_{\mathrm{ph}}$, and (d) the operator $T_{1}$. The matrix elements $\langle\alpha|O| \alpha\rangle$ are plotted agains the eigenenergy $E_{\alpha} / E_{\mathrm{av}}$. The diagonal elements for $L=6$ (red dots) are plotted in the background, those for $L=7$ (dark blue dots) in the middle, and those for $L=8$ (light blue dots) in the foreground. The arrows in (a) represent the reduced fluctuations of the diagonal elements as the system size is increased. The parameters in the Hamiltonian are $\omega_{0} / t_{0}=1 / 2, \gamma / t_{0}=1 / \sqrt{2}$ and $M_{\mathrm{ph}}=3$. Figure from Ref. [70].

it is already approximately 0.9 at $\eta=2 / 3$. The Figs. 9.3(a)-(d) show an exponential decay as a function of the $L$ for both the average fluctuation $\langle z\rangle_{\eta}(O)$ and the maximum fluctuation $z_{\max }(O)$ for all the observables studied here. This is true for $\eta=2 / 3$ and also for $\eta=1 / 3$. This result suggests that all eigenstates in the bulk of the spectrum of the Holstein polaron model are thermal in the ETH sense. 

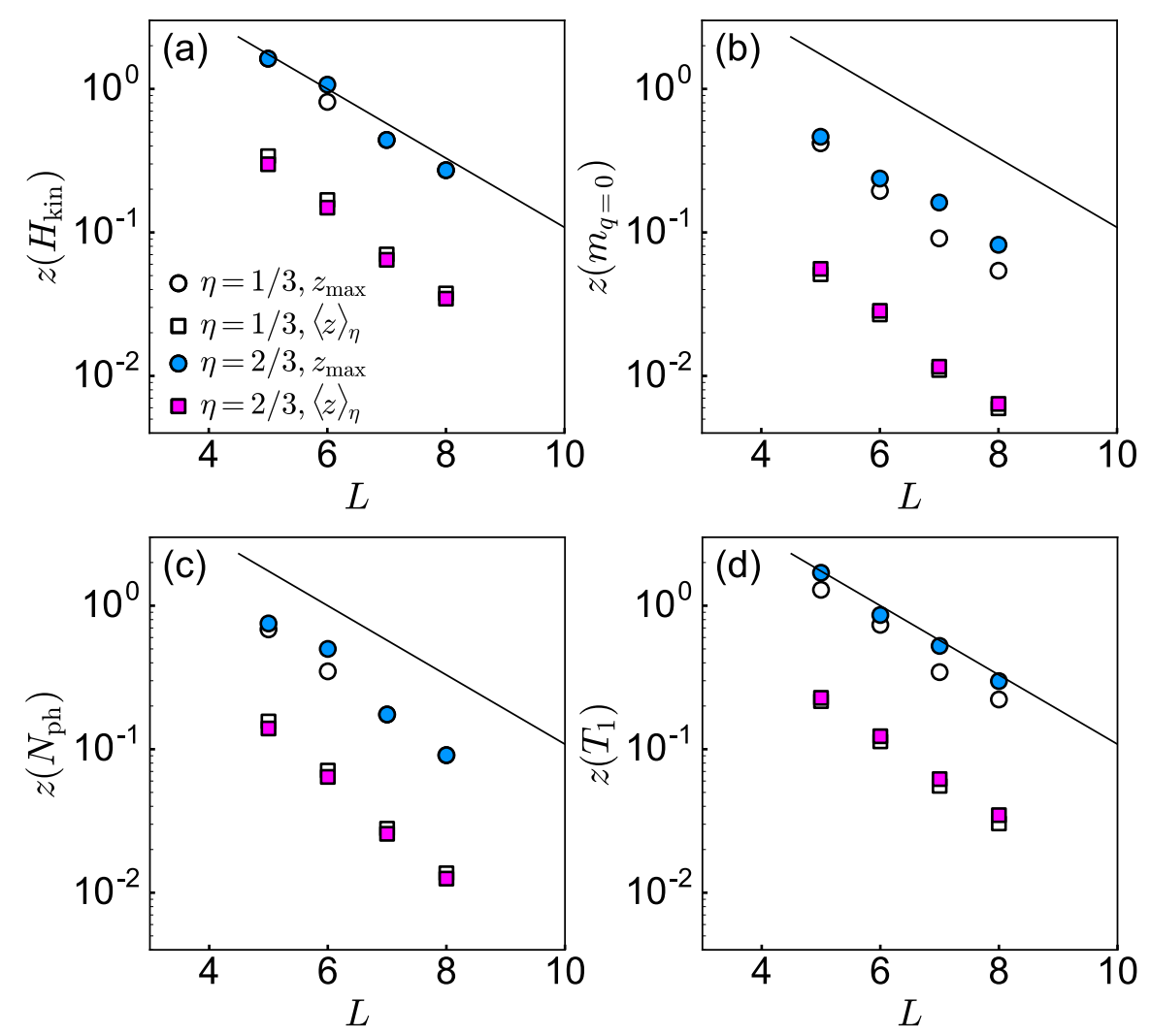

Figure 9.3: Average of the eigenstate-to-eigenstate fluctuations $\langle z\rangle_{\eta}(O)$ and maximum fluctuation $z_{\max }(O)$ in the set $\mathcal{Z}_{\eta}$ with $\eta=1 / 3,2 / 3$ as a function of the system size for (a) the fermion kinetic energy $H_{\text {kin }}$, (b) the quasimomentum occupation at $q=0, m_{q=0}$, (c) the average phonon number in the system $N_{\mathrm{ph}}$, and (d) the operator $T_{1}$. All eigenstates from the symmetry sectors $0 \leq k \leq \pi / L$ are included. The parameters in the Hamiltonian are $\omega_{0} / t_{0}=1 / 2, \gamma / t_{0}=1 / \sqrt{2}$ and $M_{\mathrm{ph}}=3$. The solid lines are guides to the eyes, representing an exponential decay. Figure from Ref. [70].

\subsubsection{Offdiagonal elements of observables}

In this section the offdiagonal matrix elements of observables $\langle\alpha|O| \beta\rangle$ with $\alpha \neq \beta$ are analysed. The eigenstates are restricted to the symmetry sector $k=2 \pi / L$ with $\mathcal{D}^{\prime}=\left(M_{\mathrm{ph}}+1\right)^{L}$ states. The pairs of eigenstates that are considered have a similar average energy $\bar{E}$. For example, if the set of pairs is restricted such that $\bar{E} \approx E_{\mathrm{av}}$, the the matrix elements cover a region of the matrix similar to the shaded region in Fig. 9.4.

In Fig. 9.5(b), the matrix elements $\left|\left\langle\alpha\left|H_{\text {kin }}\right| \beta\right\rangle\right|$ of the kinetic energy are plotted against the energy difference $\omega$ in such a region (only the matrix 

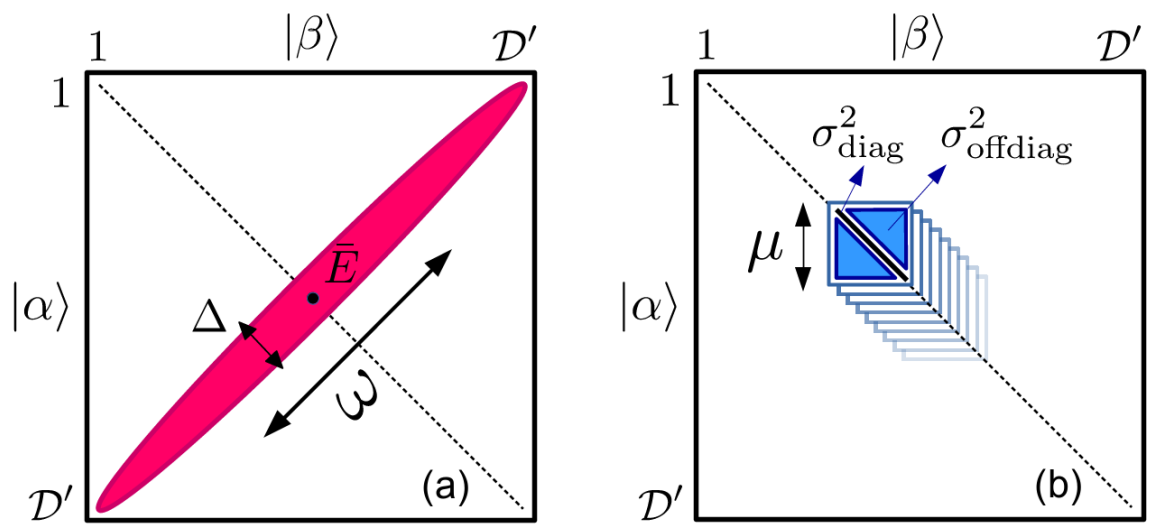

Figure 9.4: Sketch of a matrix with dimension $\mathcal{D}^{\prime}$. (a) The shaded region represents the matrix elements included in the sum in Eq. (9.22). (b) The highlighted domain indicates the sets of matrix elements included in the variances Eq. (9.24) and Eq. (9.26). Figure from Ref. [70].

elements with $\omega>0$ are plotted). The set is defined by the energy window $\left|\left(E_{\alpha}-E_{\beta}\right) /\left(2 E_{\mathrm{av}}\right)-1\right|<\Delta / 2$ with $\Delta=10^{-3}$. The strong fluctuations of the matrix elements are a manifestation of the random factor $R_{\alpha \beta}$ in Eq. (9.7). The moving average of the matrix elements, plotted as a dashed line in Fig. 9.5(b), shows an almost flat behavior for small $\omega / E_{\mathrm{av}}$ and a fast decay for larger $\omega / E_{\mathrm{av}}$ which is consistent with previous studies of offdiagonal matrix elements of observables in the two-dimensional transverse field Ising model [66] and the hard-core boson model with dipolar interactions [160].

The prefactor for the offdiagonal matrix elements from the ETH ansatz Eq. (9.7) is $\mathrm{e}^{-S(\bar{E}) / 2}$. In the bulk of the spectrum the entropy of a microcanonical energy window should scale as the Hilbert-space dimension $\mathcal{D}^{\prime}$ and therefore the prefactor should scale as $\sim 1 / \sqrt{\mathcal{D}^{\prime}}$. To check this prediction the average offdiagonal matrix element is analysed:

$$
\overline{\left|O_{\alpha \beta}\right|}=\frac{1}{\mathcal{N}} \sum_{\substack{\alpha, \beta ; \alpha \neq \beta \\\left|\left(E_{\alpha}+E_{\beta}\right) /\left(2 E_{\mathrm{av}}\right)-\bar{\varepsilon}\right|<\Delta / 2}}|\langle\alpha|O| \beta\rangle|,
$$

where $\mathcal{N}$ is the number of matrix elements included in the sum and $\bar{\varepsilon}=$ $\bar{E} / E_{\mathrm{av}}$. In Fig. 9.5(a) the average offdiagonal matrix elements of the different observables are plotted against the subspace dimension $\mathcal{D}^{\prime}$ at $\bar{\epsilon}=1$ for $\Delta=$ $10^{-1}$ (open circles) and for $\Delta=10^{-3}$ (filled symbols). It is evident that the width $\Delta$ of the energy window has almost no influence on the position of the data points. The lines in Fig. 9.5(a) are fits to the data with a fitting function $a\left(\mathcal{D}^{\prime}\right)^{-b}$. It turns out that the fit for all observables is consistent 

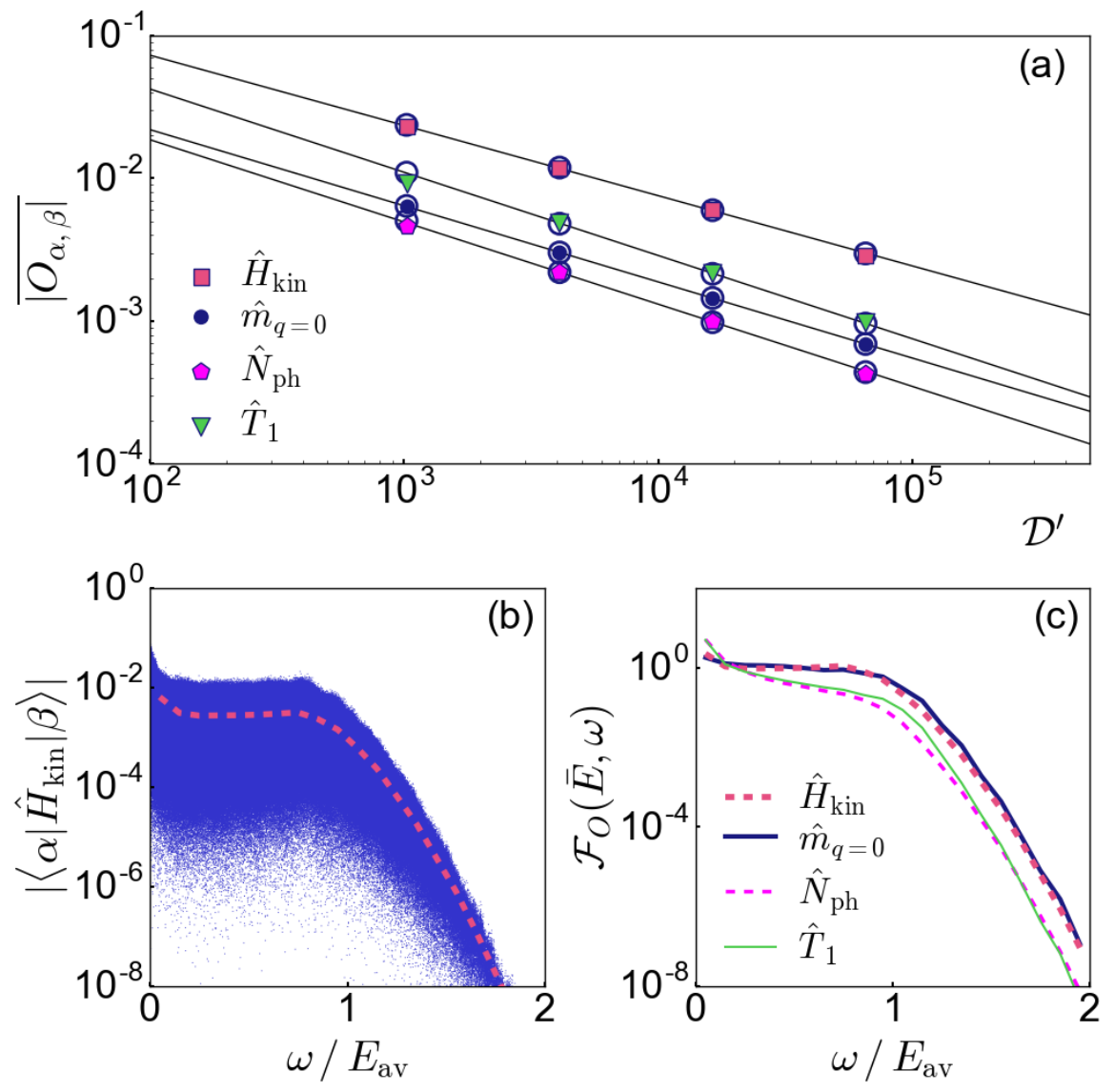

Figure 9.5: (a) Average offdiagonal matrix elements of the operators $H_{\text {kin }}$, $m_{q=0}, N_{\mathrm{ph}}$, and $T_{1}$ in the eigenbasis of the Hamiltonian as a function of the subspace dimension $\mathcal{D}^{\prime}$. The averages are calculated at $\bar{\epsilon}=1$ for $\Delta=10^{-1}$ (open circles) and for $\Delta=10^{-3}$ (filled symbols). The solid lines are fits to the data with the fitting function $a\left(\mathcal{D}^{\prime}\right)^{-b}$ for $L \geq 6$ and $\Delta=10^{-3}$. (b) Offdiagonal matrix elements of the kinetic energy $\left|\left\langle\alpha\left|H_{\text {kin }}\right| \beta\right\rangle\right|$ in the eigenbasis of the Hamiltonian as a function of $\omega$ in the energy window defined by $\bar{\varepsilon}=1$ and $\Delta=10^{-3}$. The dashed red line is the moving average of the matrix elements. (c) Moving average $\mathcal{F}_{O}(\bar{E}, \omega)$, Eq. (9.23), for the observables $H_{\text {kin }}$, $m_{q=0}, N_{\mathrm{ph}}$, and $T_{1}$ in the energy window defined by $\bar{\epsilon}=1$ and $\Delta=10^{-3}$ as a function of $\omega$. The parameters in the Hamiltonian are $\omega_{0} / t_{0}=1 / 2$, $\gamma / t_{0}=1 / \sqrt{2}$ and $M_{\mathrm{ph}}=3$. Eigenstates are restricted to the symmetry sector $k=2 \pi / L$ and $L=8$ in (b) and (c). Figure from Ref. [70].

with the prediction $b=1 / 2$ from Eq. (9.7).

To study universal properties of the function $f_{O}(\bar{E}, \omega)$ from Eq. (9.7) the 

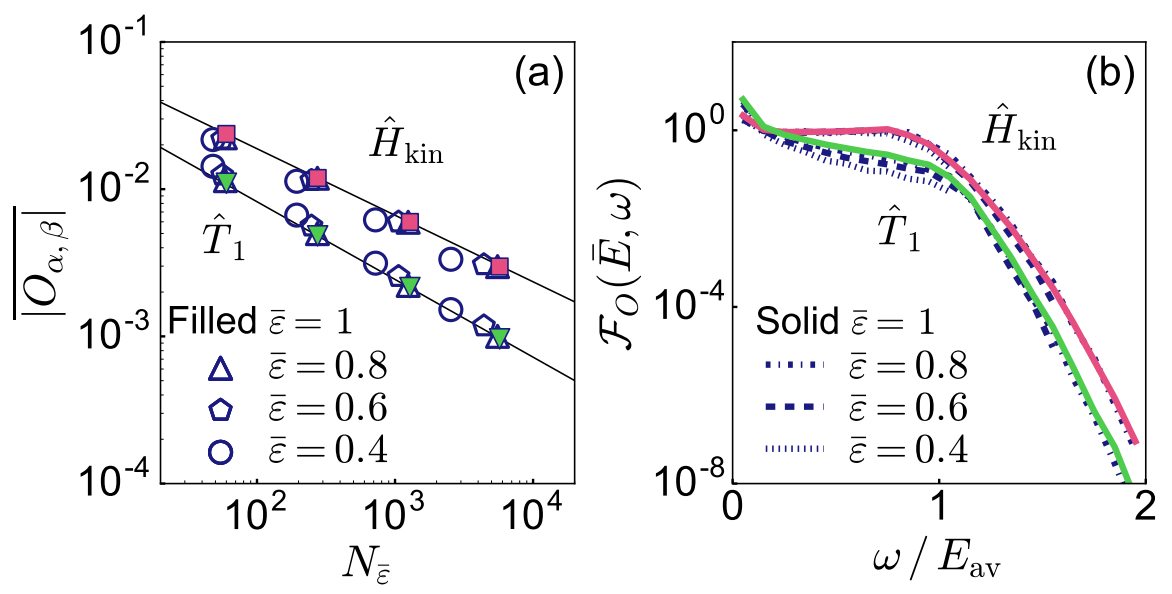

Figure 9.6: (a) Average offdiagonal matrix elements of the operators $H_{\text {kin }}$ and $T_{1}$ in the eigenbasis of the Hamiltonian as a function of the number of states in the energy window $N_{\bar{\varepsilon}}$. Different symbols correspond to different target energy densities $\bar{\varepsilon}$ and $\Delta=10^{-1}$ is used. The solid lines are fits to the data with the fitting function $a\left(\mathcal{D}^{\prime}\right)^{-b}$ for $L \geq 6$ and $\bar{\varepsilon}=1$. (b) Moving average $\mathcal{F}_{O}(\bar{E}, \omega)$, Eq. (9.23), for the observables $H_{\text {kin }}$ and $T_{1}$ as a function of $\omega$. Different line styles correspond to different target energy densities $\bar{\varepsilon}$ and $\Delta=10^{-1}$ is used. The moving average is calculated by using a window $\delta \omega / E_{\mathrm{av}}=0.1$. The parameters in the Hamiltonian are $L=8, \omega_{0} / t_{0}=1 / 2$, $\gamma / t_{0}=1 / \sqrt{2}$ and $M_{\mathrm{ph}}=3$. Eigenstates are restricted to the symmetry sector $k=2 \pi / L$. Figure from Ref. [70].

system size dependence through the subspace dimension $\mathcal{D}^{\prime}$ and randomness have to be eliminated from the matrix elements $\langle\alpha|O| \beta\rangle$. To do so the moving average of the matrix elements can be defined as that is, up to a constant factor, identical to $f_{O}(\bar{E}, \omega)$ of Eq. (9.7):

$$
\mathcal{F}_{O}(\bar{E}, \omega)=\operatorname{MA}\left(\frac{|\langle\alpha|O| \beta\rangle|}{\overline{\left|O_{\alpha \beta}\right|}}\right) \text {. }
$$

The moving average is plotted in Fig. 9.5(c) as a function of the energy difference $\omega / E_{\mathrm{av}}$ for the different observables. The scaling of the function with $\omega$ is very similar for both the observables from the electron and phonon sector.

A possible dependence of the results in Fig. 9.5(a)-(c) on the target energy density $\bar{\varepsilon}$ is studied in Fig. 9.6(a) and (b). The analysis is focused on the two observables $H_{\text {kin }}$ and $T_{1}$ from both electron and phonon sector. In Fig. 9.6(a) the average offdiagonal matrix elements $\overline{\left|O_{\alpha \beta}\right|}$ are plotted against the number 
of states $N_{\bar{\varepsilon}}$ in the microcanonical window that is analysed. This is done for different target energy densities $\bar{\varepsilon}=1,0.8,0.6,0.4$ using $\Delta=10^{-1}$. As the target energy density is moved away from the middle of the spectrum the number of states in the energy window $[\bar{\varepsilon}-\Delta / 2, \bar{\varepsilon}+\Delta / 2]$ decreases. The ETH ansatz Eq. (9.7) predicts the prefactor $\mathrm{e}^{-S(\bar{E}) / 2}$ for the offdiagonal matrix elements, where in this case $S(\bar{E})$ is exactly the logarithm of the number of states in the energy window. The data for the different $\bar{\varepsilon}$ almost collapse in Fig. 9.6(a) and the fits with the function $a\left(N_{\bar{\varepsilon}}\right)^{-b}$ yield results close to $b=0.5$ here, as well.

The dependence of the function $\mathcal{F}_{O}(\bar{E}, \omega)$ on the pair energy $\bar{E}$ is studied in Fig. 9.6(b). The results suggest that there is actually no dependence on $\bar{E}$ in case of the Holstein model, which gives numerical support for the assumption of a slow varying $\mathcal{F}_{O}(\bar{E}, \omega)$ with $\bar{E}$ in Ref. [161].

\subsubsection{Variances of diagonal vs. offdiagonal matrix ele- ments}

The variance of an observable $O$ in a set of consecutive diagonal matrix elements is:

$$
\left[\sigma_{\text {diag }}^{(\alpha, \mu)}(O)\right]^{2}=\left\langle\left(O_{\alpha \alpha}\right)^{2}\right\rangle_{\mu}-\left\langle O_{\alpha \alpha}\right\rangle_{\mu}^{2}
$$

where $\alpha$ is the first index and $\mu$ is the number of matrix elements in the set the expectation values are taken over, such that:

$$
\left\langle O_{\alpha \alpha}\right\rangle_{\mu}=\frac{1}{\mu} \sum_{\rho=\alpha}^{\alpha+\mu-1}\langle\rho|O| \rho\rangle .
$$

The variance of the offdiagonal matrix elements of $O$ is correspondingly defined as:

$$
\left[\sigma_{\text {offdiag }}^{(\alpha, \mu)}(O)\right]^{2}=\left\langle\left|O_{\alpha \beta}\right|^{2}\right\rangle_{\mu}-\left|\left\langle O_{\alpha \beta}\right\rangle_{\mu}\right|^{2}
$$

where:

$$
\left\langle O_{\alpha \beta}\right\rangle_{\mu}=\frac{1}{\mu^{2}-\mu} \sum_{\substack{\rho, \rho^{\prime}=\alpha \\ \rho \neq \rho^{\prime}}}^{\alpha+\mu-1}\langle\rho|O| \rho\rangle
$$

The sets of matrix elements considered in both variances is sketched in Fig. 9.4(b). To every microcanonical window defined by $(\alpha, \mu)$ there is a corresponding mean energy $e_{\alpha, \mu}=\left\langle H_{\alpha \alpha}\right\rangle_{\mu}$. 

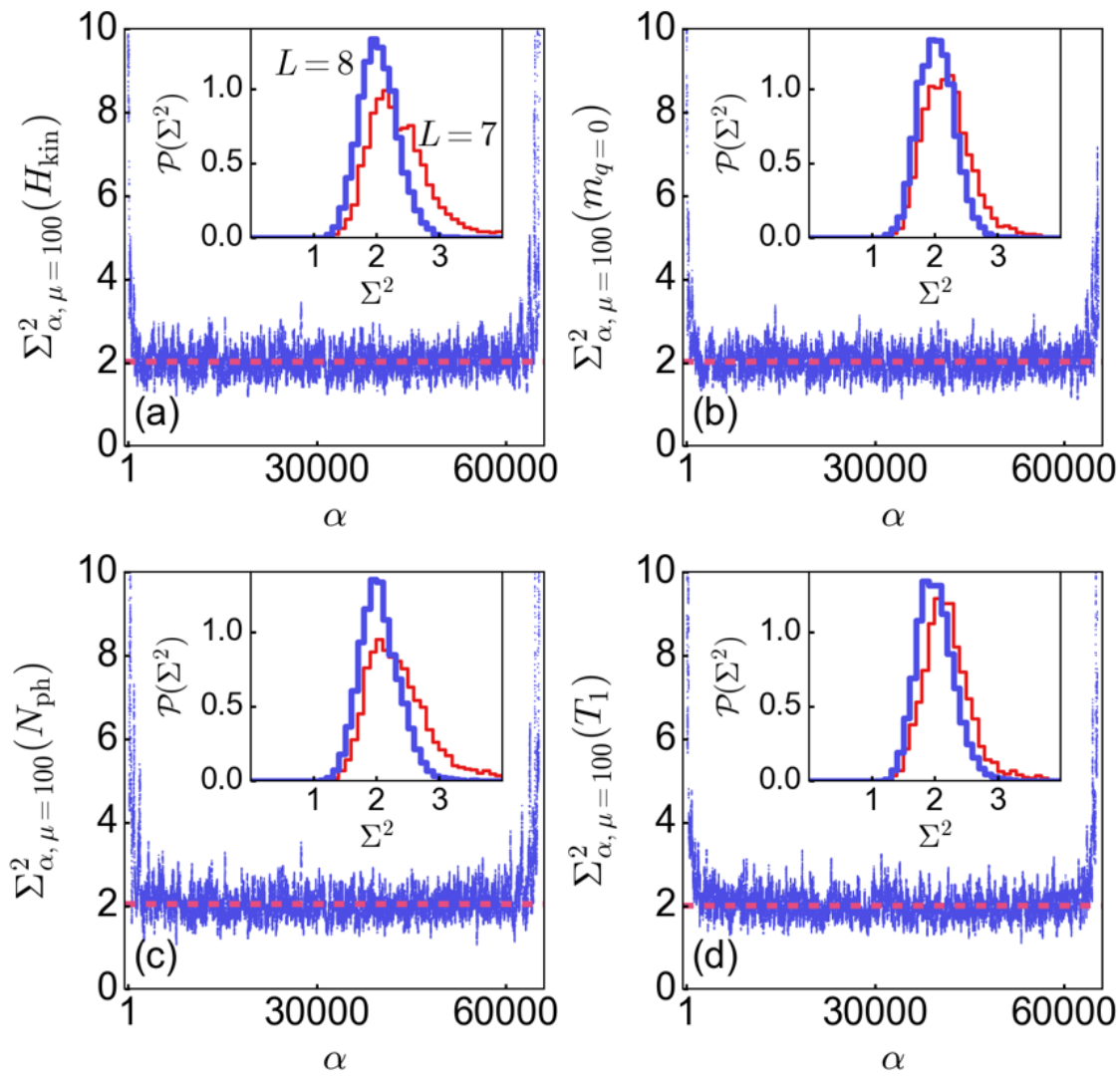

Figure 9.7: Ratio of variances between diagonal and offdiagonal matrix elements $\Sigma_{\alpha, \mu}^{2}(O)$, Eq. (9.28), for the observables (a) $H_{\text {kin }}$, (b) $m_{q=0}$, (c) $N_{\mathrm{ph}}$, and (d) $T_{1}$ as function of the index $\alpha$ for $\mu=100$. The dashed red line is the average in the of the ratios calculated from all $\alpha$ with $e_{\alpha, \mu=100} / E_{\mathrm{av}} \in$ $[1 / 3,5 / 3]$. The insets show a histogram of all the values in the set the average is calculated from, as well as, a histogram for $L=7$ and the set defined as before. The parameters in the Hamiltonian are $L=8, \omega_{0} / t_{0}=1 / 2$, $\gamma / t_{0}=1 / \sqrt{2}$ and $M_{\mathrm{ph}}=3$. Eigenstates are restricted to the symmetry sector $k=2 \pi / L$. Figure from Ref. [70].

The ratio of the variances is:

$$
\Sigma_{\alpha, \mu}^{2}(O)=\frac{\left[\sigma_{\text {diag }}^{(\alpha, \mu)}(O)\right]^{2}}{\left[\sigma_{\text {offdiag }}^{(\alpha, \mu)}(O)\right]^{2}} .
$$

The prediction from random matrix theory is that $\Sigma_{\mathrm{GOE}}^{2}(O)=2$ for generic local observables in the GOE [62]. The question is then whether this value can be found in real physical systems that obey the ETH and for which part 
of the spectrum [162].

For the analysis done in Fig. 9.7(a)-(d) $\mu=100$ is fixed and the $\Sigma_{\alpha, \mu}^{2}(O)$ is calculated for all $\alpha \in\left[1, \mathcal{D}^{\prime}-\mu\right]$. The system size is $L=8$ and $M_{\mathrm{ph}}=3$ which yields a Hilbert-space dimension $\mathcal{D}^{\prime}=65536$ and, therefore, $\mu \ll \mathcal{D}^{\prime}$. These numbers are consistent with the numbers used in a corresponding analysis of observables in the two-dimensional transverse field Ising model in Ref. [66]. One can see that for all observables considered here the ratio of variances is close to 2 in a wide range of energies in the bulk of the spectrum.

The dashed red line the four panels shows the average of $\Sigma_{\alpha, \mu=100}^{2}(O)$ over all $\alpha$ for which $e_{\alpha, \mu=100} / E_{\mathrm{av}} \in[1-\eta, 1+\eta]$ and $\eta=2 / 3$ is used. The averages are 2.05, 2.04, 2.07, and 2.03 for Figs. 9.7(a)-(d) respectively, which is in very good agreement with the GOE prediction. The histograms in the insets of Figs. 9.7(a)-(d) show the distribution of $\Sigma_{\alpha, \mu=100}^{2}(O)$ in the set used for the calculation of the average. Additionally a histogram of the $\Sigma_{\alpha, \mu=100}^{2}(O)$ for $L=7$ and $M_{\mathrm{ph}}=3$ is shown where again $e_{\alpha, \mu=100} / E_{\mathrm{av}} \in[1-\eta, 1+\eta]$ and $\eta=2 / 3$ is used. One can observe how the increase of system size leads to a distribution that is peaked sharper and that the maximum approaches the GOE prediction 2 .
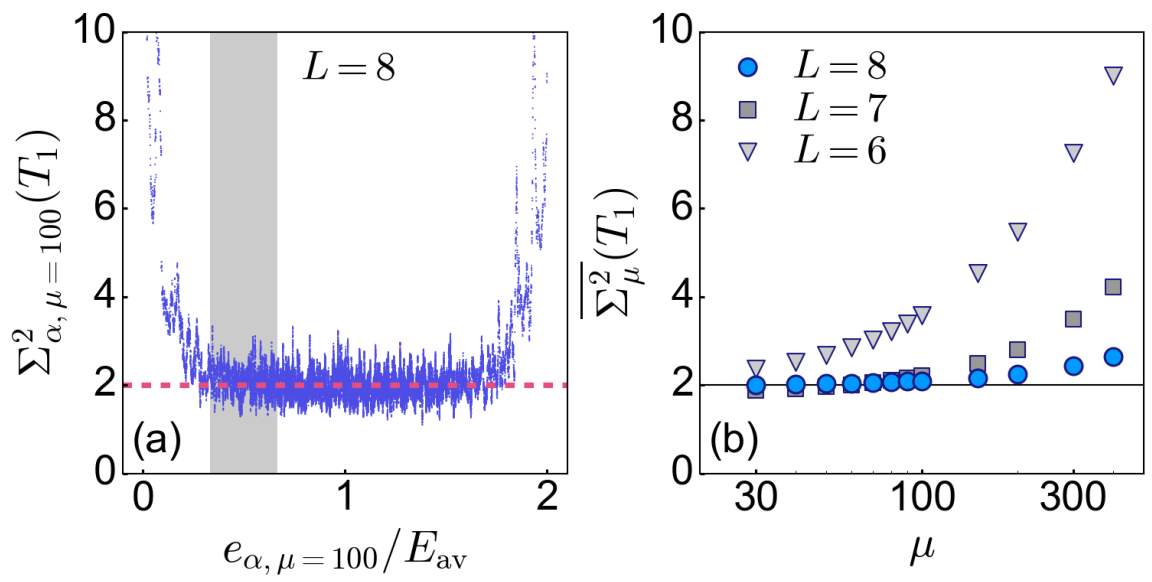

Figure 9.8: (a) Ratio of variances between diagonal and offdiagonal matrix elements $\Sigma_{\alpha, \mu}^{2}(O)$, Eq. (9.28), for the observables $T_{1}$ as function of the mean energy $e_{\alpha, \mu}$ where $\mu=100$. (b) Average ratio of variances $\overline{\Sigma_{\mu}^{2}}(O)$ for the observables $T_{1}$ as function of the parameter $\mu$ for different system sizes $L=$ $6,7,8$. The shaded region in panel (a) is the region that is averaged over in (b). The parameters in the Hamiltonian are $\omega_{0} / t_{0}=1 / 2, \gamma / t_{0}=1 / \sqrt{2}$ and $M_{\mathrm{ph}}=3$. Eigenstates are restricted to the symmetry sector $k=2 \pi / L$ and $L=8$ in (a). Figure from Ref. [70].

Figure 9.7(a)-(d) shows very good agreement to the GOE prediction for 
a vast number of $\alpha$ values also away from the middle of the spectrum. However, the presentation of the data might be a bit misleading since the spectrum is denser in the middle. Therefore, Fig. 9.8(a) shows the same data as Fig. $9.7(\mathrm{~d})$ on the $y$-axis but the $x$-axis shows the mean energy $e_{\alpha, \mu=100}$, here. The data reveals a clear disagreement with the GOE prediction at the very edges of the spectrum only. For a large portion of the spectrum towards the center of it $\Sigma_{\alpha, \mu=100}^{2}\left(T_{1}\right) \approx 2$. This observation might be surprising since eigenstates towards the boundary of the spectrum are no complete random superpositions of basis states from a simple basis, while such randomness is expected for states in the center of the spectrum $[62,156,163]$.

To study the dependence of $\Sigma_{\alpha, \mu}^{2}$ on $\mu$ and the systems size the average is calculated:

$$
\overline{\Sigma_{\mu}^{2}}(O)=\frac{1}{\left\|\mathcal{S}_{\mu}\right\|} \sum_{\Sigma_{\alpha, \mu}^{2} \in \mathcal{S}_{\mu}} \Sigma_{\alpha, \mu}^{2}(O),
$$

where the sum runs over the set:

$$
\mathcal{S}_{\mu}=\left\{\Sigma_{\alpha, \mu}^{2}: e_{\alpha, \mu} / E_{\mathrm{av}} \in[1 / 3,2 / 3]\right\} .
$$

The set is sketched in Fig. 9.8(a) as the shaded region. In Fig. 9.8(b) the average $\overline{\Sigma_{\mu}^{2}}\left(T_{1}\right)$ is plotted against the parameter $\mu$ for different system sizes $L=6,7,8$ and $M_{\mathrm{ph}}=3$. The results show that as the system size is increased larger portions of the spectrum can be included in the calculation of $\Sigma_{\alpha, \mu}^{2}$ to still yield an agreement with the GOE prediction. Furthermore, the results are consistent with the assumption that $\Sigma_{\alpha, \mu}^{2} \rightarrow \Sigma_{\mathrm{GOE}}^{2}$ as $L \rightarrow \infty$ even very close to the edge of the spectrum.

\subsection{Summary}

To summarize, eigenstate thermalization and quantum chaos in the Holstein polaron model was studied in this chapter. The model serves as an example for a solid-state like closed quantum system which features an electron on a lattice coupled to phonons. To this end, the Hamiltonians of systems with up to $L=8$ sites and up to $M_{\mathrm{ph}}=3$ phonons per site were diagonalized numerically and the spectrum of eigenenergies as well as matrix elements of observables in the Hamiltonian eigenbasis were analyzed.

The level spacing statistics shows characteristics of matrices from the Gaussian orthogonal ensemble indicating quantum chaotic behavior. This is true for a wide range of coupling strengths $\gamma / t_{0}$ and phonon frequencies $\omega_{0} / t_{0}$ and even persists if the maximum number of phonons per site is restricted 
to $M_{\mathrm{ph}}=1$. Analysing the behavior for different system sizes with fixed $M_{\mathrm{ph}}$ gives indications for quantum chaos and the ETH in the limit $L \rightarrow \infty$ while the thermodynamic limit would correspond to taking both the limits $M_{\mathrm{ph}} \rightarrow \infty$ and $L \rightarrow \infty$. These findings strengthen the conjecture from previous numerical studies that a non extensive integrability breaking term of $O(1)$ is actually enough to induce quantum ergodicity and thermalization [71-76].

Further, the matrix elements of different observables in the eigenbasis of the Hamiltonian were analysed and their distribution was compared to the ETH ansatz [61]. The investigation of the distribution of diagonal elements shows a convergence towards a smooth function of the energy. This conclusion was drawn from the exponential decay of eigenstate-to-eigenstate fluctuations as a function of the system size.

The dependence of the 'universal' part of the offdiagonal elements on the energy difference between pairs of eigenstates is comparable for all considered observables. Moreover, there is at most a very weak dependence on the average energy. The chapter closes with an analysis of the ratio between fluctuations of diagonal and offdiagonal elements. Here, the predictions from random matrix theory are met in a broad energy window around the center of the spectrum. Combined, the results show robust features of thermalization in the ETH sense. 


\section{Chapter 10}

\section{Sudden expansion in the 1d Fermi-Hubbard model}

In the previous chapters different phenomena in the 1d Holstein model were discussed and studied numerically. The model consists of a simple tightbinding chain coupled to a phonon branch and is designed to study the influence of phonons on the properties of non-interacting electrons in a simple model system, while the direct interaction between electrons is neglected.

In this chapter, the Fermi-Hubbard model is studied which is a different model system designed to study the direct interaction of the electrons amongst each other [164], while lattice vibrations are ignored. In $1 d$ the Hamiltonian is:

$$
H_{\mathrm{FH}}=-J \sum_{l, \sigma=\uparrow, \downarrow}\left(c_{l \sigma}^{\dagger} c_{l+1 \sigma}+c_{l+1 \sigma}^{\dagger} c_{l \sigma}\right)+U \sum_{l} n_{l \uparrow} n_{l \downarrow},
$$

where $c_{l \sigma}^{\dagger} / c_{l \sigma}$ are creation/annihilation operators of electrons with spin $\sigma$ at site $l$ and $n_{l \sigma}=c_{l \sigma}^{\dagger} c_{l \sigma} . \quad J$ is the hopping matrix element and $U$ the onsite interaction strength.

The work presented here is published in Ref. [79]. It contains the study of transport properties in the 1d Fermi-Hubbard model by means of a sudden expansion setup in a quantum-gas experiment. The results of the experiment are compared to tDMRG calculations. The author contributed to the production of the numerical data in all figures and in particular the analysis of the velocities in Figs. 10.6, 10.7, and 10.8. Furthermore, he was involved in the discussions, interpretation of the experimental data, and the writing of the paper including the figures.

Quantum-gas experiments have become a powerful tool to study the physics of model Hamiltonians in a controlled environment [77, 78]. One unique aspect of these experiments is the possibility to explore the influence 

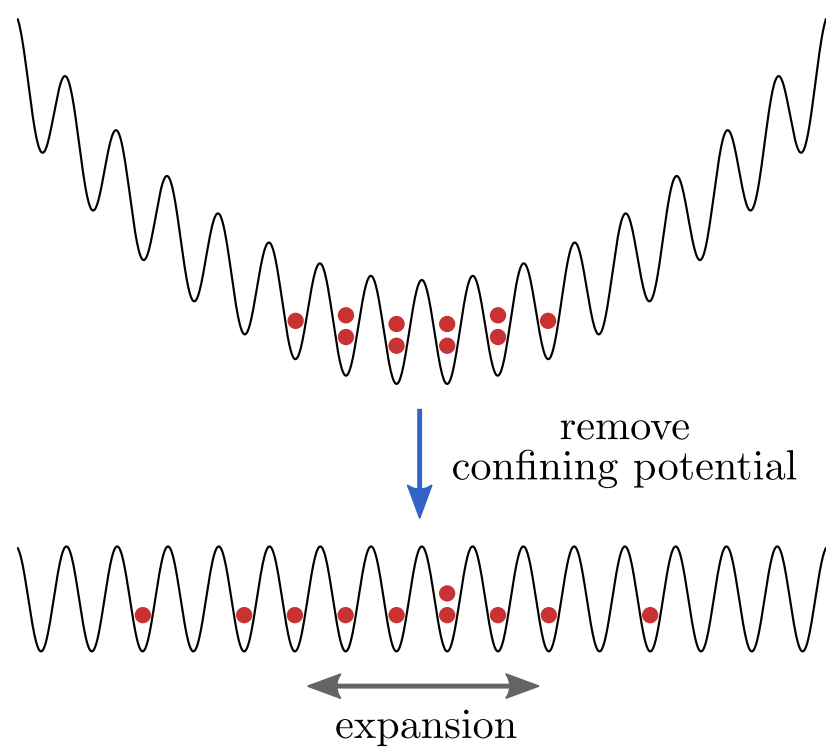

Figure 10.1: Sketch of the protocol to initiate the dynamics in a sudden expansion experiment. The particles are prepared in a trapping potential that keeps them in the center of an optical lattice. When the trapping potential is removed, the particles can expand into the empty lattice.

of dimensionality on the properties of quantum systems, by realizing $1 \mathrm{~d}, 2 \mathrm{~d}$, or $3 \mathrm{~d}$ geometries and even tune between those [80,165].

1d systems differ from higher dimensional ones in several aspects. Importantly, Fermi liquid theory breaks down in 1d [166] and numerous integrable models exist that cease to thermalize [167-169]. The realization of the integrable Tonks-Girardeau gas [170] and the observation of the peculiar dynamics in this system [67] demonstrate the capabilities of quantum gas experiments to study low dimensional systems.

In an optical lattice, transport properties can be investigated by means of so called sudden expansion experiments [79-81,171]. The particles in the lattice are initially confined to a limited space. This may be achieved by a harmonic trapping potential. The dynamics is initialized by removing the trapping potential while keeping the optical lattice in place. As a result, the atoms tunnel into the empty lattice sites (the experimental protocol is sketched Fig. 10.1). The impact of dimensionality and Hamiltonian parameters on the mass transport may be studied by tuning the lattice depth and interaction strength. Furthermore, different initial states can be realized (close to product states or correlated states) by varying the loading procedure $[79-81,171]$.

The work presented here and in Ref. [79] is the first realization of a sudden 
expansion of fermions in 1d. It is motivated by earlier experiments on the Fermi-Hubbard model in higher dimensions [171] and on the Bose-Hubbard model $[80,81]$. The results of these earlier experiments are summarized in the following, before the results of Ref. [79] are recapitulated.

\subsection{Earlier sudden expansion experiments in the Fermi- and Bose-Hubbard model}

In Ref. [171], Schneider et al. realized the sudden expansion of fermions. The initial state is prepared such that the particles are localized at single sites and the density distribution in the center of the cloud is flat. Crucially, the initial state is independent of the parameters in the Hamiltonian during the expansion. After the confining potential in the horizontal directions is turned off, the particles start spreading into empty lattice sites as a result of the density gradient. The effect of different values of the interaction parameter $U / J$ (cf. Eq. (10.1)) on the spreading of the cloud is studied by measuring the real space density of the particles.

In the non-interacting case the spreading of the localized particles is ballistic. The effect here is that the initially spherical cloud inherits the lattice geometry and turns into a square shaped cloud.

In the interacting case the behavior is different. The particles in the outer part of the cloud still spread ballistically since the density in this region is low and, therefore, the interaction does not have a large impact. The inner part of the cloud constitutes a higher density core. In this core, the interactions lead to diffusive spreading which is slow compared to the ballistic spreading of the outer cloud part. For the largest interaction strengths investigated a shrinking of the core is observed. This is explained by a slow melting of the core. Particles that detach from the core escape ballistically. Furthermore, a symmetry with respect to the sign of the interaction is observed. In contrast to the behavior in free space, the dynamics for attractive and repulsive interaction are identical.

A similar experiment was conducted by Ronzheimer et al., Ref. [80], but in this case the Bose-Hubbard model was realized in the optical lattice in $1 \mathrm{~d}$ and 2d:

$$
H_{\mathrm{BH}}=-J_{x} \sum_{\langle l, m\rangle_{x}} b_{l}^{\dagger} b_{m}-J_{y} \sum_{\langle l, m\rangle_{y}} b_{l}^{\dagger} b_{m}+\frac{U}{2} \sum_{l} n_{l}\left(n_{l}-1\right),
$$

where $b_{l}^{\dagger}\left[b_{l}\right]$ are bosonic creation [annihilation] operators at site $l$ and $J_{x}\left[J_{y}\right]$ are the hopping matrix elements. The first two sums run over the nearest neighbors in the $x$ and $y$ direction, respectively, and in the case of $1 \mathrm{~d} J_{y}=0$. 
Again, atoms are initialized such that they are localized in the central part of the lattice. The state can be described by local Fock states:

$$
\left|\psi_{0}\right\rangle=\prod_{l \in \text { trap }} \frac{1}{\sqrt{\nu_{l} !}}\left(b_{l}^{\dagger}\right)^{\nu_{l}}|\emptyset\rangle,
$$

where $\nu_{l} \in\{0,1,2, \ldots\}$. Depending on the loading procedure, there are only single occupied sites (singlons) or there can be sites with occupation $>1$ (doublons, triplons, ...), while the average density is one (unit filling).

The central quantity studied in this work is the spreading velocity of the core $v_{\mathrm{c}}$. It is obtained by following the half width at half maximum (HWHM) of the measured density distribution in time. For intermediate times, the time evolution of the HWHM is linear and $v_{\mathrm{c}}$ is calculated from fitting a straight line to this linear part.

If there are only single occupied sites in the initial state the key observations are as follows: In the non-interacting case the spreading is again ballistic in both $1 \mathrm{~d}$ and $2 \mathrm{~d}$. This is the case, where the fastest expansion appears. When the interaction is turned on, the expansion velocity is reduced monotonically as a function of $U$ in $2 \mathrm{~d}$. In $1 \mathrm{~d}$, however, $v_{\mathrm{c}}$ is reduced for small interactions and then increases again for larger interactions. The slowing down is explained by the dynamical generation of higher occupations. Although these dynamically generated doublons and triplons (and higher occupations) are not completely stable, they nevertheless move slower and act as impurities where singlons can scatter. A further effect is the transformation of kinetic energy into interaction energy in the formation of higher occupations, which is another explanation of the slowing down. For very large interactions $U / J_{x / y}$ the Bose-Hubbard model approaches the hard core limit $U \rightarrow \infty$. In $1 \mathrm{~d}$, the hard core boson model can be mapped to non-interacting spinless fermions, which show again ballistic spreading. Therefore, the expansion velocity approaches the value from the non-interacting case for large $U / J_{x}$ in 1 d.

By tuning the ratio $J_{y} / J_{x}$ one can investigate the crossover from $1 \mathrm{~d}$ to $2 \mathrm{~d}$. In fact, the core velocities interpolate between the two different cases. In the $2 \mathrm{D}$ case the behavior is very similar to the Fermi-Hubbard case studied in Ref. [171] where a diffusively expanding round core has a background of a ballistically expanding dilute cloud with an emerging square shape.

As a third scenario the admixture of higher occupations into the initial state in $1 \mathrm{~d}$ is studied. While there is no effect on the expansion velocity in the non-interacting case, the higher occupations decrease the velocities with increasing interaction strength $U / J_{x}$. The effect is articulated for very large $U / J_{x}$. In this case, multi occupations can be seen as stable objects that can 
only move with a very small effective hopping in an empty lattice. The stability comes from the fact that the interaction energy has to be transformed into kinetic energy if a multi occupation is broken up. In a situation where the interaction energy is larger than the bandwidth of the constituents, this leads to stable objects that can only be broken up in higher order processes. Similarly, the hopping to a neighboring site of a multi occupation requires higher order processes that are suppressed by the interaction strength. Therefore, the high occupations stay at their original positions on the timescales of the experiment while the single occupations can escape.

\subsubsection{Quantum distillation}

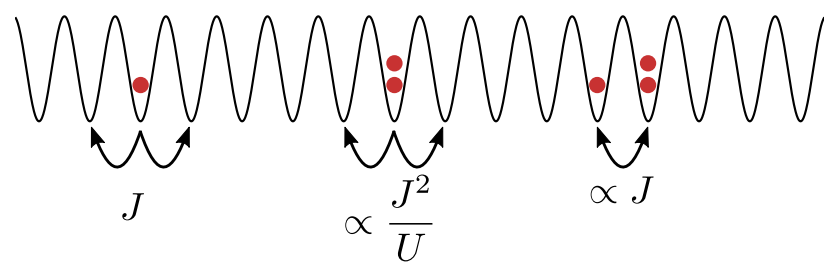

Figure 10.2: Sketch of the basic hopping mechanisms in the Fermi- and Bose-Hubbard model. Singlons can hop to empty neighboring sites with amplitude $J$. In the limit $U \gg 4 J$, doublons can hop to neighboring sites with an effective hopping amplitude $J_{\text {eff }} \propto J^{2} / U$. Singlons and doublons on neighboring sites can swap places with a hopping amplitude $\propto J$.

The effects mentioned above can even lead to the multi occupations moving toward the center of the cloud, a phenomenon called quantum distillation [81-84]. It can be understood from the basic hopping mechanisms in the Fermi- and Bose-Hubbard model sketched in Fig. 10.2. Singlons move through the empty lattice with the hopping $J$. Doublons are more or less immobile for large $U / J$ (they move with an effective hopping $\propto J^{2} / U$ through an empty lattice). However, a doublon and a singlon on neighboring sites can exchange places with the hopping $J$ in the Fermi-Hubbard model and with $2 J$ in the Bose-Hubbard model. Therefore, doublons can move rather freely on a singlon background but get stuck when no singlons assist them. If a mixed cloud of singlons and doublons expands this leads to the doublons being concentrated (distilled) in the center of the cloud while the singlons escape from the edges of the cloud. The effect was suggested as a means of producing low entropy states in quantum-gas experiments [82]. 


\subsubsection{Probing quantum distillation in the 1d Bose- Hubbard model}

Quantum distillation in the 1d Bose-Hubbard model was studied in a quantumgas experiment by Xia et al. in Ref. [81]. In contrast to the previous experiments, the particles were not prepared in local Fock states in this case. Instead, the quench involved the trapping potential only, so that the particles were in local equilibrium after the quench.

As mentioned above, singlons in the Bose-Hubbard model move with a hopping matrix element $J$ through an empty lattice, while singlons and doublons exchange places with the matrix element $2 J$. The doubled bandwidth within a doublon core means that singlons can only escape if they sit in the middle of the band. In contrast, the hopping matrix elements for singlons in the Fermi-Hubbard model is equal inside and outside a doublon block and therefore singlons can escape easier. It is therefore expected that quantum distillation is more effective in the Fermi-Hubbard model than in the Bose-Hubbard model [81,83].

Three different interaction strengths were studied in Ref. [81]. The cores of the cloud contain a mixture of singlons and higher occupations where most of the higher occupations are doublons. Again, one can observe that only the singlons escape from the core and spread ballistically with a velocity independent of the interaction strength, as expected. The doublons (and triplons) in the core stay at their position on the timescales of the experiment but the density of the doublons slowly decreases. The higher the interaction $U / J$ the more stable the doublons (and triplons) are.

For all three interaction strengths doublons and triplons dissociate into singlons in the early stages of the expansion. However, in later stages doublons are stable in case of the largest interaction. In this case quantum distillation effects become important. The number of singlons confined in the core steadily decreases but for the latest times studied, the decrease levels off. This effect can be explained by the mismatch of the bandwidth of singlons inside and outside the core. Singlons with small quasi momentum $k$ cannot escape which inhibits quantum distillation.

In the time evolution of the doublon-cloud width (measured as full width at half maximum) there are three stages; first an increase, because doublons expand on the singlon background while they dissolve as well; then a decrease because of quantum distillation. In case of the largest interaction, the width after the distillation stage is rather stable, while for the smaller interactions the shrinking is overtaken by expansion because higher occupations eventually dissolve. 


\subsection{Sudden expansion of a fermion cloud}

As mentioned above, fermionic quantum distillation is expected to be more effective than its bosonic counterpart. Furthermore, it could be used for cooling by generating low entropy states in the center of a fermionic cloud. This idea is rather intriguing since evaporative cooling, which is the standard cooling techniques to ultra cold temperatures in quantum-gas experiments, is less effective for fermions than it is for bosons [78].

The experimental protocol in Ref. [79] is similar to the one in Ref. [80]. The atoms are prepared in a deep optical lattice that is superimposed by a harmonic potential. The loading procedure is such that the initial state is close to a product state of the form:

$$
\left|\psi_{0}\right\rangle=\prod_{i \in \operatorname{trap}}\left(c_{i \uparrow}^{\dagger}\right)^{\nu_{i \uparrow}}\left(c_{i \downarrow}^{\dagger}\right)^{\nu_{i \downarrow}}|\emptyset\rangle
$$

where $\nu_{i \sigma} \in\{0,1\}$.

The dynamics is started by turning off the confining harmonic potential and adjusting the lattice depth in such a way that a certain interaction strength $U / J$ is accomplished. After these adjustments the atom cloud that was initially confined to the center of the lattice spreads out into the empty lattice sites according to the Fermi-Hubbard Hamiltonian Eq. (10.1).

After a waiting time, the density at the sites is frozen out by ramping up the lattice depth. Then an in situ image of the cloud is taken. By varying the waiting time in repeated experiments, the dynamics of the system can be mapped out. The doublons can be removed or not before the imaging, so that the spreading of singlons and doublons can be followed separately [79].

To obtain the integrated line densities $\rho_{\mathrm{s}}\left[\rho_{d}\right]$ of singlons [doublons] the image is integrated in one spatial direction. Together with the imaging this means that the integrated line densities are sums over many $1 d$ systems. From these line densities the singlon radius $R_{\mathrm{s}}$ and doublon radius $R_{\mathrm{d}}$ can be extracted as HWHM [80].

Two different initial states are considered. In the first case [cf., Fig. 10.3(a)], singlons and doublons as well as holons (non occupied sites) are present in the initial atom cloud. In this case, quantum distillation is expected to appear in the large $U / J$ limit. Doublons move toward the center of the cloud, while singlons escape to the empty lattice. However, holons that are trapped in between doublons are expected to weaken the quantum distillation.

In the lower part of Fig. 10.3(a) a numerical (tDMRG) simulation of the dynamics is shown. In $x$-axis direction the position on a $1 \mathrm{~d}$ chain is plotted, while on the $y$-axis the time is plotted in units of the tunneling time $\tau=1 / \mathrm{J}$. 
(a) Initial state with doublons

(b) Initial state without doublons
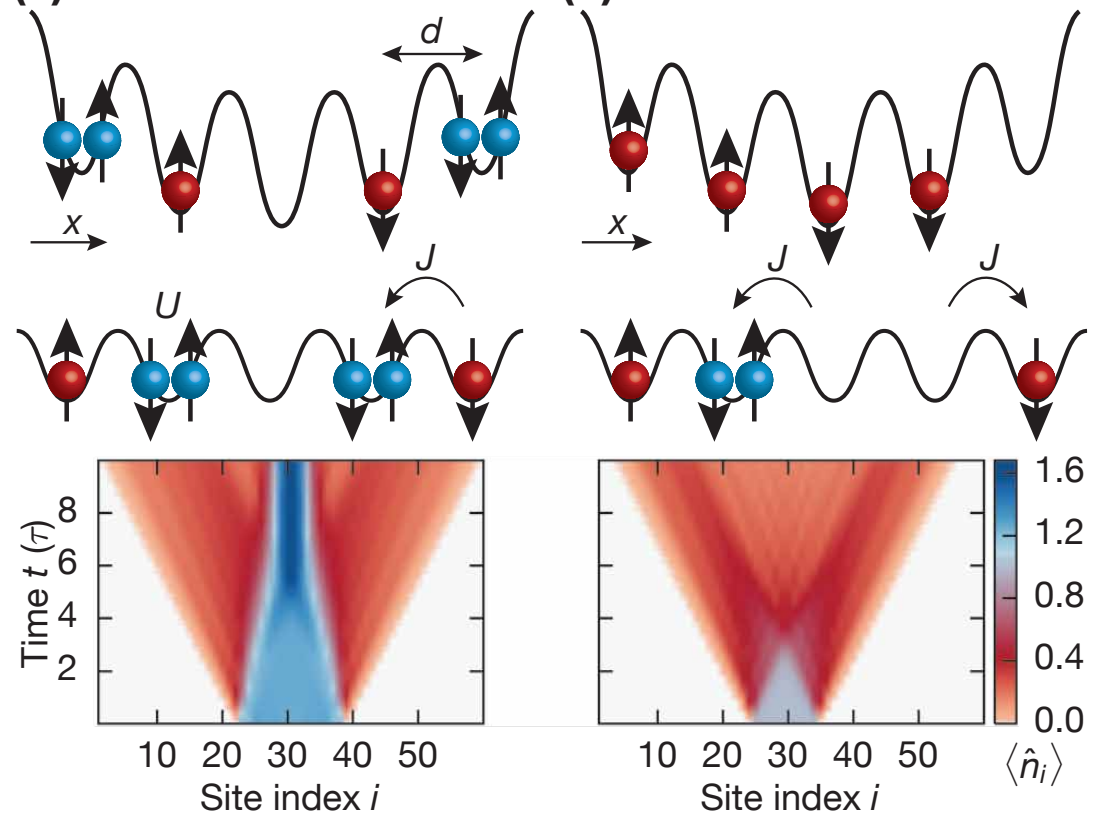

Figure 10.3: Sketch of the experimental protocol in Ref. [79]. (a) Initial state with a mixture of singlons, doublons and holons. After the confining potential is removed and the interaction strength is quenched to a finite value, doublons and singlons can exchange places with the hopping amplitude $J$. The fact that singlons escape at the edges of the cloud lets the doublons concentrate in the center. Holons can prevent a further concentration of the doublons. In the lower part, a tDMRG simulation of an expanding singlondoublon mixture at $U / J=20$ is shown. The colorcode represents the density $\left\langle n_{i}\right\rangle$ as a function of the lattice site $i$ on the $x$-axis and the time on the $y$-axis. A high density core evolves, while singlons travel outwards. (b) Initial state with only singlons. After the quench, doublons emerge dynamically and the cloud expands into the empty lattice. The lower part displays a tDMRG simulation of a pure singlon cloud at $U / J=20$. Figure from Ref. [79]. (c) 2018 American Physical Society

The color represents the density. At $U / J=20$ the doublons in the initial cloud get concentrated in the center, while the singlons fly out.

In the second case [cf., Fig. 10.3(b)], only singlons are present in the initial particle cloud. Upon release, doublons are generated dynamically while the cloud expands. In this case, the dependence of the expansion velocity on the interaction strength is studied. Again, a numerical simulation of the initial state without doublons at $U / J=20$ is shown in the lower part of 
Fig. 10.3(b).

\subsubsection{Expansion with doublons in the initial state}

Figures 10.4(a)-(d) show experimental results from the expansion of a fermion cloud with a mixture of singlons and doublons in the initial state with a doublon fraction of $n_{\mathrm{d}}=0.40(2)$. The doublon fraction $n_{\mathrm{d}}$ is the fraction of particles on double occupied sites defined as:

$$
n_{\mathrm{d}}=\frac{N_{\mathrm{d}}}{N_{\mathrm{s}}+N_{\mathrm{d}}}
$$

where $N_{\mathrm{s}}\left[N_{\mathrm{d}}\right]$ is the number of particles on single [double] occupied sites. For the data in Fig. 10.4(a) the interaction strength is set to $U / J=5$. The radius of the singlon cloud $R_{\mathrm{s}}$ and the radius of the doublon cloud $R_{\mathrm{d}}$ is plotted as a function of the expansion time as red and blue dots, respectively.

It is evident that the singlon cloud expands more rapidly than the doublon cloud. The dynamics of the doublon cloud is roughly comparable to the expansion of non-interacting particles with an effective hopping amplitude $J_{\text {eff }}=2 J^{2} / U$ [172] plotted as a dashed blue line in Fig. 10.4(a).

The dynamic separation of singlons and doublons is even more pronounced at $U / J=20$ [cf., Fig. 10.4(b)]. The time evolution of the singlons radius is not vastly different from the one at $U / J=5$. However, the doublon radius shrinks in this case giving a first indication towards quantum distillation happening. To exclude the possibility of the doublon cloud shrinking happening because of a simple melting of the doublon cloud, the number of singlons and doublons as a function of time is plotted in the insets of Figs. 10.4(a) and (b). At $U / J=5$, the number of particles on double occupied sites shrinks over the course of the first 5 tunneling times, while the number of particles on single occupied sites increases by the same amount. After this initial conversion of doublons into singlons is finished the numbers stay constant. In contrast, the number of singlons and doublons stay roughly constant over the full expansion time in case of the larger interaction $U / J=20$ such that the shrinking of the doublon cloud cannot be explained by melting.

To get a better impression of what happens in the clouds at $U / J=20$, the integrated line densities of singlons and doublons are presented in Fig. 10.4(b) in the initial state $(t=0)$ on the left and at $t=40 \tau$ on the right. The singlon cloud expands over the course of the expansion time while a change of the doublon cloud is barely visible.

The scenario of quantum distillation is further supported by Fig. 10.4(c) where the ratio of particles on doublon and singlon sites in a central region 

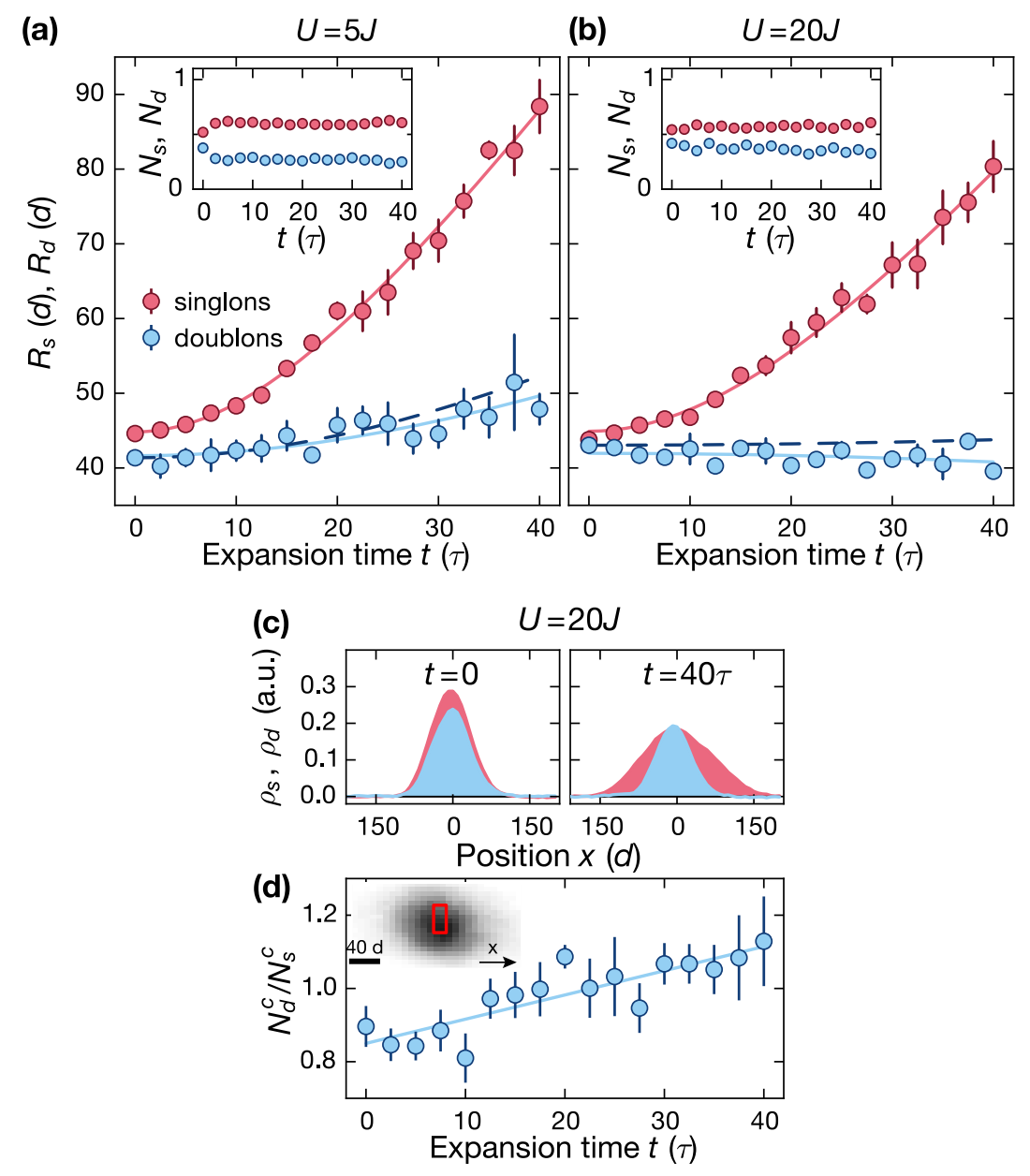

Figure 10.4: Experimental results for the expansion of a singlon-doublon mixture. (a) Singlon radius $R_{\mathrm{s}}$ (red) and doublon radius $R_{\mathrm{d}}$ (blue) as a function of time at $U / J=5$. The blue dashed line represents the expansion of a cloud of non-interacting fermions with a hopping matrix element $J_{\text {eff }}=$ $2 J^{2} / U$. The solid lines are guides to the eye. The inset shows the number of particles on single and double occupied sites as a function of time. (b) Same as in (a) but for $U / J=20$. (c) Integrated line density of singlons and doublons in the initial state (left) and at $t=40 \tau$ (right) at $U / J=20$. (d) Ratio of the number of particles on double and single occupied sites in a central region of the cloud (marked region in the inset) as a function of time at $U / J=20$. The solid line is a guide to the eye. To obtain a datapoint four measurements are averaged and the standard error of the mean is calculated yielding the error bars. Figure from Ref. [79]. (c) 2018 American Physical Society 


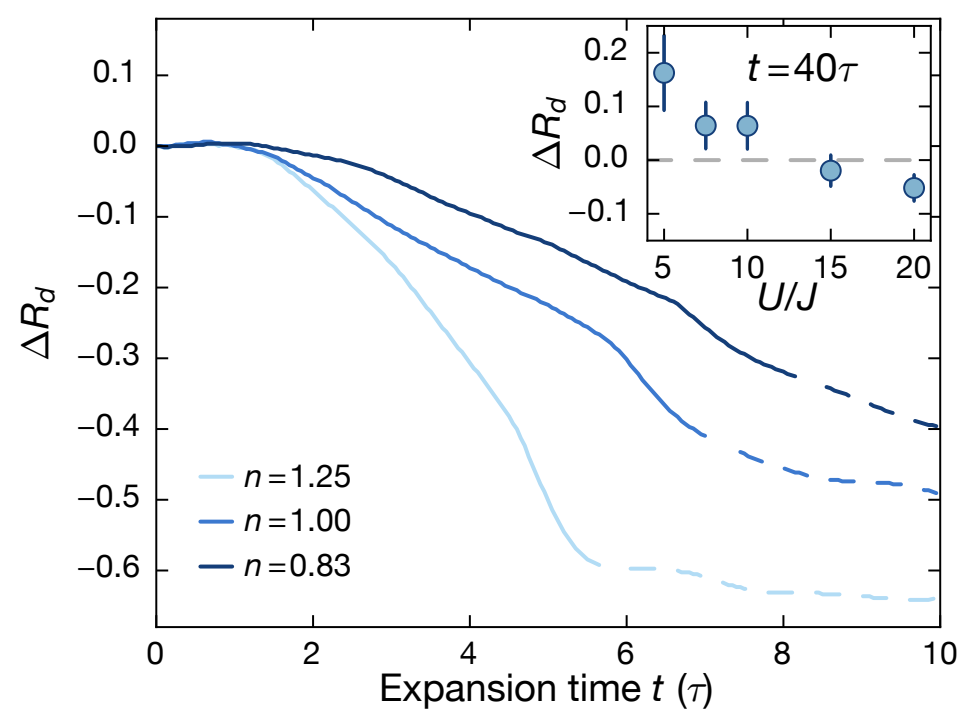

Figure 10.5: Relative change in doublon radius $\Delta R_{\mathrm{d}}$ as a function of time at $U / J=20$ obtained from tDMRG simulations. The different colors represent different densities in the initial cloud. The lower densities are accomplished by introducing holons into the initial cloud. The transition from solid to dashed line marks the time when the singlon cloud has doubled in size. Inset: Relative change in doublon radius at $t=40 \tau$ measured in the experiment as a function of the interaction strength $U / J$. Figure from Ref. [79]. (C) 2018 American Physical Society

(the red box in the inset) is plotted as a function of time. The ratio monotonically increases as doublons move toward the center of the cloud while singlons move outwards. 1d systems with a low doublon density at the edges of the cloud could provide an alternative explanation of the increased doublon to singlon ratio. This effect is, however, too small to account for the signal seen in Fig. 10.4(c) [79].

The occurrence of the quantum distillation effect, crucially depends on the interaction strength $U / J$. Doublons become stable objects if the interaction energy is considerably larger than the bandwidth $(W \ll U$, where $W=4 J)$ [173].

The relative change in the doublon cloud radius can be defined as:

$$
\Delta R_{\mathrm{d}}(t)=\frac{R_{\mathrm{d}}(t)}{R_{\mathrm{d}}(t)}-1
$$

This quantity at $t=40 \tau$ is plotted in the inset of Fig. 10.5 as a function of $U / J$. As already anticipated from Fig. 10.4(a) and (b), the doublon cloud 
expands for small interaction strengths. Only for the largest interaction strength $U / J=20$ studied here, a clear shrinking of the doublon cloud is observed.

The influence of the initial cloud density on the efficiency of quantum distillation is studied by means of numerical tDMRG simulations in the main panel of Fig. 10.5. Different from the experiment, an exact box trap for the preparation of the initial state is assumed. A compact core of only singlons and doublons with $n_{\mathrm{d}}=0.4$ yields a particle density of $n=1.25$. The lower densities are achieved by adding additional holons in the core. In the experiment the exact distribution of singlons and doublons in the initial state is random and the measuring process averages over many $1 \mathrm{~d}$ tubes. To mimic this situation in the numerics, every dataset in Fig. 10.5 is an average over 120 samples of random distributions of singlons, doublons and holons in the core where the spin of the singlons is also random. The distributions are chosen such that the average over the initial states yields a flat density distribution in the core.

Introducing holons into the initial state influences the shrinking of the doublon core in two ways. First of all, the efficiency of the quantum distillation is reduced. Holons in between doublons inhibit their ability to move further toward the center and, therefore, the reduction of the radius is diminished as the density is reduced. Furthermore, the speed of the shrinking is reduced in the lowerd density cases. This is important for the interpretation of the experimental data. The maximum time in the experiment is limited. As the singlons travel to the outer parts of the lattice, they reach regions where the lattice is not completely flat anymore. The maximum time in the experiment is given by the time when the singlon cloud radius is approximately doubled [see Fig.10.4(a) and (b)]. In the numerical data in Fig. 10.5 this is indicated by the change from a full to a dashed line. In case of the lower densities, $\Delta R_{\mathrm{d}}$ is still decreasing when the singlon cloud has doubled in size. Both of the above observations suggest that the efficiency of quantum distillation is reduced in the experiment.

\subsubsection{Expansion without doublons in the initial state}

In this section, the effect of the interaction strength $U / J$ on the asymptotic expansion velocity is studied for the case of no doublons in the initial state (see Fig. 10.3(b)). For that purpose, the second moment of the time dependent density distribution is calculated:

$$
r^{2}=\sum_{l} \rho_{l}\left(l_{c}-l\right)^{2} 8.3^{2} d^{2},
$$




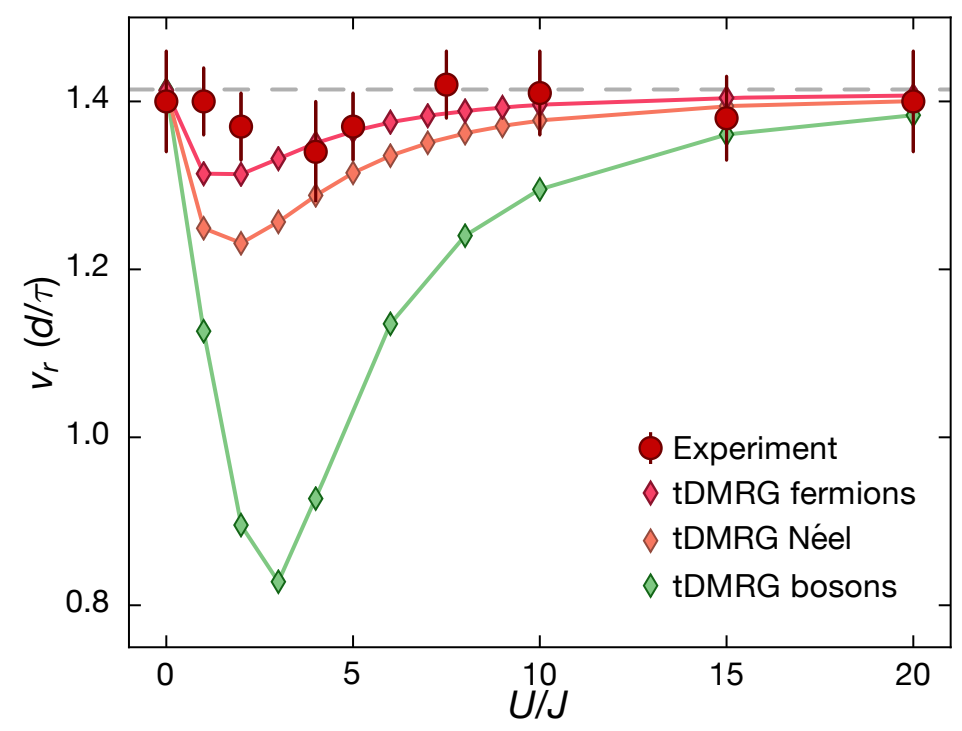

Figure 10.6: Radial velocities $v_{r}$ for a cloud without initial doublons as a function of the interaction strength $U / J$. The dark red points are results from the experiments. The diamonds are tDMRG results with $N=10$ particles. The red diamonds are results for the full average over all spin configurations. The orange diamonds represent results for a initial Néel state. The green diamonds are results for the Bose-Hubbard model. The grey dashed line represents the exact result $v_{r}=\sqrt{2} d / \tau$ in the non-interacting and hard core limit. Figure from Ref. [79]. (c) 2018 American Physical Society

where $\rho_{l}$ is the normalized density at a pixel extracted from the in situ images, $l_{c}$ is the central pixel, $d$ is the lattice constant, and every pixel is 8.3 lattice sites wide. The second moment is better suited for a comparison with numerical data since it takes into account the full density distribution. Therefore, it is less affected by the initial cloud shape and size than the core radii $R_{\mathrm{s}}$ and $R_{\mathrm{d}}[79,80]$. The asymptotic radial velocity $v_{r}$ is then extracted by fitting the function $\sqrt{r^{2}}=\sqrt{r_{0}^{2}+v_{r}^{2} t^{2}}$ to the data, where $r_{0}$ is the initial cloud radius.

The radial velocities measured in the experiment are plotted against the interaction strength $U / J$ as red dots in Fig. 10.6. In the non-interacting limit the measured velocity is compatible with the expected analytical value $\sqrt{2}$. Upon increasing the interaction the velocity shows a slight dip at $U / J \approx 4$. For even larger $U / J$ the velocity approaches again the value $\sqrt{2}$ which is expected in the hard core limit [174].

The experimental data is compared to numerical tDMRG data. As before, a box trap is assumed for the numerical simulations. The initial state 


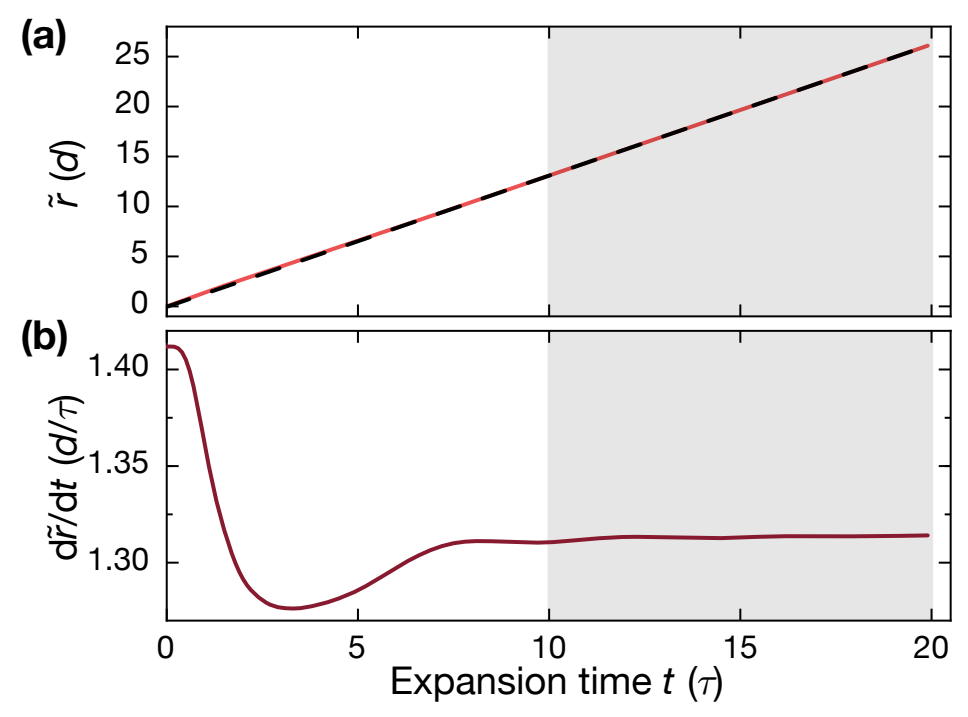

Figure 10.7: Fitting procedure to obtain the radial velocity from the tDMRG data. (a) The reduced radius $\tilde{r}(t)$ as a function of time (see Eq. (10.9)) as red solid line and the linear fit as black dashed line. (b) Numerical time derivative of $\tilde{r}(t)$ as a function of time. The shaded region indicates the fitting window. Figure from Ref. [79]. (c) 2018 American Physical Society

containes $N=10$ particles with equal amount of up and down spins in the center of a $L=100$ site system. For every possible spin configuration with $N_{\uparrow}=N_{\downarrow}$ a separate simulation is run and the densities are then averaged over all spin configurations.

The time dependent reduced radius $\tilde{r}(t)$ is calculated as:

$$
\begin{aligned}
r^{2}(t) & =\frac{1}{N} \sum_{i=1}^{L}\left\langle n_{i}\right\rangle(t)\left(i_{0}-i\right)^{2}, \\
\tilde{r}(t) & =\sqrt{r^{2}(t)-r^{2}(0)},
\end{aligned}
$$

where $\left\langle n_{i}\right\rangle(t)$ is the time dependent density at site $i$ averaged over all spin configurations.

The fitting procedure to obtain the velocity $v_{r}$ from the linear part of $\tilde{r}(t)$ is illustrated in Fig. 10.7(a) and (b). The time evolution of $\tilde{r}(t)$ is plotted in Fig. 10.7(a) and the numerical time derivative of $\tilde{r}(t)$ in Fig. 10.7(b). The shaded region in Fig. 10.7(b) indicates the fitting window where the velocity has settled to a basically constant value.

The velocities calculated in this fashion are plotted in Fig. 10.6 as red diamonds. As a function of the interaction strength, $v_{r}$ first decreases until 


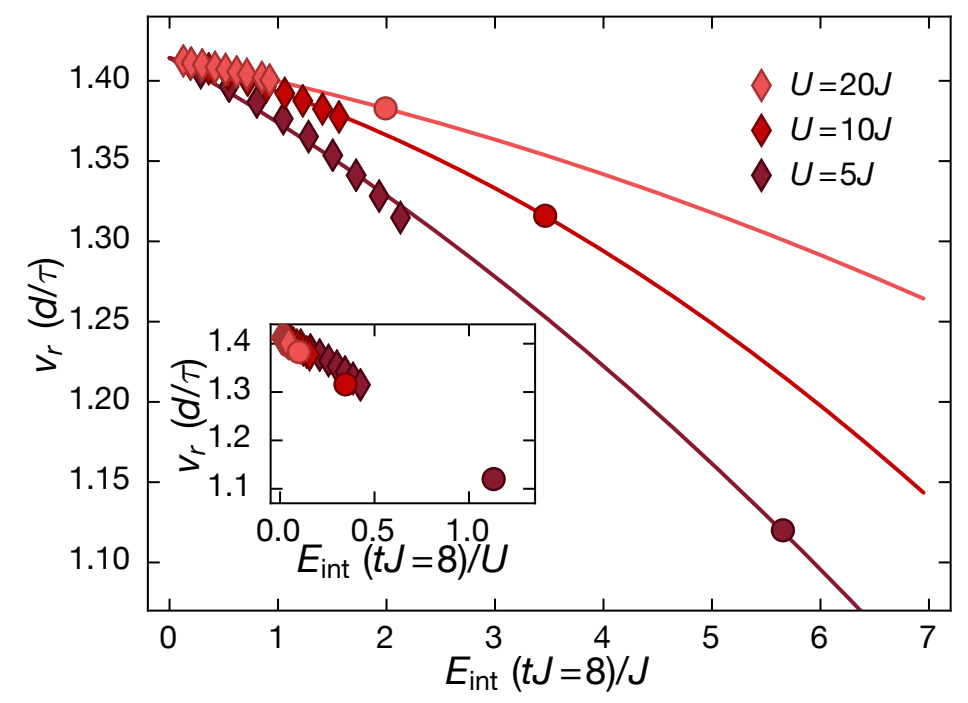

Figure 10.8: Radial velocity $v_{r}$ obtained from tDMRG simulations as a function of the interaction energy $E_{\text {int }}$ generated in the quench. Different colors represent different interactions strengths $U / J$. The different diamonds with the same color represent different numbers of up-down neighbors in the initial cloud in case of the Fermi-Hubbard model. The dots are results for the Bose-Hubbard model. Inset: Same data as the main panel but the interaction energy on the $x$-axis is plotted in units of the interaction strength $U$. Figure from Ref. [79]. (C) 2018 American Physical Society

$U / J \approx 1.5$ and then it increases again to approach the asymptotic value $\sqrt{2}$ in the hard core limit. The experimental results are overall compatible with the numerical predictions.

The decrease in velocity is due to the dynamical generation of doublons after the quench. Doublons can only be created when singlons of opposite spin occupy neighboring sites. Therefore, the amount of velocity reduction depends on the precise spin configuration in the initial state. To backup this reasoning the velocities of the configuration with the maximum number of neighboring opposite spins (Néel state in the initial cloud) is plotted as orange diamonds in Fig. 10.6. In this case, the dip in the velocity for intermediate $U / J$ values is more pronounced. Furthermore, the velocity in the case of bosons is plotted as green diamonds in Fig. 10.6 (see also Ref. [80,174]). In the bosonic case, there is only one initial configuration since they do not carry any spin. Doublons can be generated at all bonds and, additionally, even higher occupations can be created. The result is an even stronger reduction of $v_{r}$ at intermediate coupling strengths compared to the fermionic case. 
As mentioned above, different causes for the velocity reduction are proposed in Ref. [80]. One of them is the conversion of kinetic energy to interaction energy in the quench. The reduced kinetic energy then leads to a slower expansion of the cloud. The interaction energy in the Fermi-Hubbard model is defined as:

$$
E_{\text {int }}=U \sum_{i=1}^{L}\left\langle n_{i \uparrow} n_{i \downarrow}\right\rangle
$$

where $n_{i \sigma}=c_{i \sigma}^{\dagger} c_{i \sigma}$. In the Bose-Hubbard model, the interaction energy is defined in a similar fashion:

$$
E_{\mathrm{int}}=\frac{U}{2} \sum_{i=1}^{L}\left\langle n_{i}\left(n_{i}-1\right)\right\rangle
$$

where $n_{i}=b_{i}^{\dagger} b_{i}$.

In Fig. 10.8, $v_{r}$ is plotted as a function of the interaction energy for different values of $U / J$. The different diamonds for one value of $U / J$ correspond to different amounts of up-down neighbors in the initial state where the leftmost diamond corresponds to just one neighbor and the rightmost diamond to the Néel state. The dots correspond to the Bose-Hubbard model. In the expansion the interaction energy decreases toward an asymptotic value. While our simulations do not reach the asymptotic regime, the time when $E_{\text {int }}$ is calculated is chosen so that the initial fast drop of $E_{\text {int }}$ is excluded.

A clear dependence of the velocities on $E_{\text {int }}$ can be observed in Fig. 10.8 with an additional dependence on the interaction strength $[175,176]$. Plotting the interaction energy in units of $U$ results in an almost collapse of the data (see the inset of Fig. 10.8). The $U$-dependence can be explained by the nonzero expansion velocity of the doublons at smaller $U / J$ and the fact that they are only stable for $W \ll U$. The stability and immobility of doublons are, however, the assumptions used in Ref. [64] to explain the velocity reduction in the Bose-Hubbard model.

\subsection{Summary}

In summary, this chapter was concerned with the sudden expansion of particle clouds in the 1d Fermi-Hubbard model. Starting from product states with a mixture of singlons and doublons, a dynamical phase separation between the two could be observed in a quantum gas experiment. The findings are 
consistent with the phenomenon of fermionic quantum distillation. Comparing the experimental results to tDMRG calculations, the low efficiency in the experiment could be linked to additional holons in the initial state.

When the system is initialized with only singlons, a reduced radial velocity of the cloud is observed at intermediate coupling strengths which is consistent with tDMRG calculations. The reduction of the velocity can be explained by the generation of doublons after the release of the cloud. Here, the comparison of numerical results for fermions and bosons reveals that the slowing down can be explained by a conversion of kinetic energy into interaction energy for both particle statistics. 


\section{Chapter 11}

\section{Conclusion and outlook}

The bulk part of this thesis was concerned with analysing both the ground state and time evolution of the half-filled 1d Holstein model of spinless fermions coupled to an Einstein phonon branch. DMRG in the MPS language was used to conduct the numerical calculations [40,41,111]. The large local Hilbert spaces due to the phononic degrees of freedom without number conservation pose a special challenge to the aforementioned numerical techniques. To this end, the LBO was adopted to make the MPS techniques more efficient. Within the LBO, the site tensors of a MPS are dynamically transformed into the eigenbasis of the single-site reduced density matrix and a truncation of the local basis can be performed according to the spectrum of the single-site reduced density matrix [29].

In his thesis, F. Dorfner combined the ideas by Guo et al. [49] with the single-site DMRG with subspace expansion introduced by Hubig et al. [50], to the DMRG3S+LBO suited for ground-state calculations with LBO on extended systems [48]. Here, the inner workings of this algorithm were analysed in detail, in particular the interplay between the subspace-expansion mixing factor and initial states, and their influence on the convergence was studied. It was found, that it is beneficial to first calculate the ground state for an easy case and then gradually change the parameters in the Hamiltonian from one DMRG run to the next, until the desired parameter set for a potentially more challenging case is reached.

In addition to the LBO, a new technique to deal with large local Hilbert spaces, the projected purification [37], was introduced in this work, where the author contributed to the developement. The idea within this technique is to project the state into an enlarged Hilbert space where a number conservation of the phonons can be established. For this purpose, additional phonon bath sites are introduced for every physical site. Reestablishing a global $U(1)$ symmetry results in an effective local dimension of one. 
The DMRG3S+LBO method and the PP-DMRG method were compared to the established pseudo-site DMRG ansatz by conducting ground-state calculations in the half-filled Holstein model [38]. While all methods are able to provide comparable results for all regions in the phase diagram considered, there are also notable differences. The PP-DMRG is conceptually simple and allows for the use of a standard two-site solver. This makes it straight forward to use, however, the additional bath sites cause the MPS bond dimensions to be larger for a given target precision compared to the LBO technique. In contrast, the DMRG3S+LBO utilizes a single-site solver with subspace expansion. The additional internal parameters, the mixing factor in the subspace expansion and the number of optimized local basis states kept, makes converging the code more demanding. As mentioned above, the upside of the LBO ansatz is the smaller bond dimension compared to the PP method, which is especially beneficial close to the phase transition in the Holstein model at half filling.

A TEBD-LBO ansatz [51] was then used to study the melting of CDW order in the Holstein model [54], which is the first time this method is applied to a half-filled system. The work presented here is complementary to the one by Hashimoto and Ishihara [57], where the melting of CDW order after an optical pulse in the Hubbard-Holstein model was investigated. In contrast, the pure Holstein model of spinless fermions without direct interaction between the charge carriers was considered here. Furthermore, larger quench energies occur in our work $\left(0.1 t_{0} N \leq \Delta E \leq 8 t_{0} N\right)$ compared to the excess energy pumped into the system by the optical pulse in Ref. [57] $\left(\Delta E \leq 0.1 t_{0} N\right)$.

Overall, it is found that a coupling to phonons slows down the electron dynamics due to polaron formation, similar to the results in Ref. [57]. When starting from the bare CDW state with no phonons, a transient self trapping effect can be found for large electron-phonon couplings that is analogous to the one found by Kloss et al. in Ref. [58], where the spreading of a single localized charge carrier in the Holstein model was studied. In the CDW melting scenario, the self trapping results in a cascade like decay of the order parameter, where the length of the plateaus in the cascade are given by the period of the harmonic oscillators $2 \pi / \omega_{0}$. Quenches from the CDW to the TLL phase result in different timescales for the relaxation of the electronic and phononic system. Electrons hop between sites rather freely such that the CDW order melts, while the phonons are left behind and the order in the phonon sector persists for longer times. Here, it would be interesting to explore, whether an additional phonon hopping can accelerate the relaxation of the phonon inhomogeneities.

To consider a scenario closer to experiments, one could expand this study by implementing a Peierls pulse, analogous to Ref. [57]. From a technical 
point of view this is more demanding since the Hamiltonian becomes time dependent. Therefore, the gates for the time evolution in the TEBD-LBO algorithm have to be recalculated at every time step (at least for the duration of the pulse) which becomes increasingly expensive for large $M_{\mathrm{ph}}$.

Furthermore, the exact result from the TEBD-LBO can be taken as benchmark data to test hybrid quantum-classical methods for the time evolution of electron-phonon coupled systems, where the lattice dynamics is propagated classically while the electron dynamics is treated quantum mechanically (e.g. Ehrenfest dynamics $[177,178]$ ). These methods are typically more capable concerning system size as well as timescales compared to full quantum mechanical treatments and not restricted to $1 \mathrm{~d}$. The exact quantum mechanical results of the TEBD-LBO can offer valuable insights on parameter sets and times where the hybrid methods are reliable.

Subsequently, the question of thermalization in the Holstein model was investigated. To this end, the ETH was tested in the Holstein polaron model by using exact diagonalization in small systems of up to $L=8$ sites and up to $M_{\mathrm{ph}}=3$ phonons per site. Although the integrability breaking term in the Hamiltonian does not scale with the system size, quantum chaos indicators suggest that the system thermalizes already for very small electron-phonon coupling strengths. This result holds even when the maximal phonon number per site $M_{\mathrm{ph}}$ is restricted to just one.

In the following, the ETH ansatz for observables in the eigenbasis of the Hamiltonian was examined by focusing on a single parameter set in the Hamiltonian. The considered observables were the kinetic energy and quasimomentum occupation in the fermionic sector as well as the average phonon number in the system and the nearest-neighbor offdiagonal matrix elements of the phonon one-body correlation matrix. The diagonal elements showed the expected behavior of converging to a smooth function of the energy. This was checked by looking at eigenstate to eigenstate fluctuations of the diagonal elements, which exhibit an exponential decay as a function of the system size for all eigenstates in the bulk of the spectrum.

The offdiagonal elements of the considered observables followed the ETH prediction as well and in particular the universal function $\mathcal{F}_{O}(\bar{E}, \omega)$ showed only a very slight dependence on the average energy $\bar{E}$. Furthermore, predictions from the random matrix theory were met concerning the ratio between variances of fluctuations of diagonal to offdiagonal matrix elements of observables.

Overall, the results established thermalizing behavior of the Holsteinpolaron model, where the integrability breaking term in the Hamiltonian is of 
the order $\mathcal{O}(1)[71-73,75]$. Extensions of this study might go in the direction of making the phonon system less degenerate by introducing a dispersion or disorder. In fact, Schönle et al. [152] considered the case of dispersive phonons.

The topic of the last part of this thesis approaches transport from a different angle and the perspective of ultracold quantum gas experiments. The dynamics of interacting fermions without a coupling to phonons was investigated in the Fermi-Hubbard model. Specifically, the spreading of initially confined fermion clouds into an empty 1d lattice was studied experimentally and numerically, where the initial states were prepared such that the fermions are in local Fock states [79]. In case of a mixture of singlons and doublons in the initial cloud, the phenomenon of quantum distillation [81-84] was detected in the experiment, that was previously found for bosons [80,81]. With the help of the numerics, the low efficiency of the quantum distillation in the experiment could be traced back to additional holons in the initial cloud.

When considering only singlons in the initial state, the radial velocity of the cloud depends on the onsite interaction strength $U$ between the particles, where the lowest velocities emerge for intermediate $U / J$ in $1 \mathrm{~d}[80,174]$. This phenomenon is connected to the fact that doublons are created dynamically after the release of the cloud and the emerging interaction energy is then no longer available as kinetic energy. It was established numerically that this explanations holds for both the integrable Fermi-Hubbard model and the nonintegrable Bose-Hubbard model.

As mentioned above, important steps to improve the performance of ground-state and time-dependent DMRG methods for electron-phonon coupled systems were discussed throughout this thesis. This includes the LBO as an effective ansatz both for ground-state DMRG and time-dependent DMRG. Recently, the LBO was also combined with finite-temperature DMRG [108]. Further, the newly developed PP promises to be an additional technique to tackle these problems efficiently. Here, it was used to calculate ground states, but it is also compatible with time-evolution and finite-temperature methods. It will be exciting to see how competitive PP can be in those areas. 


\section{Appendix A}

\section{Charge-density-wave melting in the Holstein model: Finite-size dependence}

Here, results for different system sizes $L$ are compared for the charge-densitywave melting discussed in Ch. 8. In Fig. A.1, data for the bare CDW melting is presented for $\omega_{0} / t_{0}=2, \gamma / t_{0}=1,3,4$, and system sizes $L=7,11,15$. Overall, there are no qualitative differences between the system sizes concerning the behavior of the order parameter for all coupling strengths considered. The largest quantitative deviations appear for $\gamma / t_{0}=1$ [Fig. A.1(a)]. For the larger coupling strengths $\gamma / t_{0}=3,4$ [Fig. A.1(b),(c)] differences are only visible after the first plateau in the order parameter for $t t_{0} \gtrsim 3$. Such a dependence of finite-size effects on the coupling strength is expected since the dynamics gets slowed down as $\gamma / t_{0}$ is increased.

This trend is even more pronounced when comparing results for different system sizes in case of the dressed CDW melting [Fig. A.2]. Discrepancies are only visible for the smallest coupling $\gamma / t_{0}=1$ [Fig. A.2(a)]. For $\gamma / t_{0}=3,4$ [Fig. A.2(b),(c)] the data points for the different $L$ lie on top of each other over the course of the entire simulation. Only very small deviations can be seen for $\gamma / t_{0}=3$ and $t t_{0} \gtrsim 5.5$. As discussed in the main text, the initial dressed CDW state is very close to the ground state for large $\gamma / t_{0}$ and, therefore, there is only very slow dynamics.

The $\mathcal{O}_{\mathrm{CDW}}$ data for the frequency and coupling quenches exhibit very little finite-size dependence as well (Fig. A.3). Here, data for $L=9,13,15$ are presented. The largest deviations are seen for the frequency quench [Fig. A.3(a)]. In this case, the electron dynamics is comparatively fast since it is fairly decoupled from the phonon dynamics. In contrast, the electron dynamics is dictated by the phonon dynamics after the coupling quench 


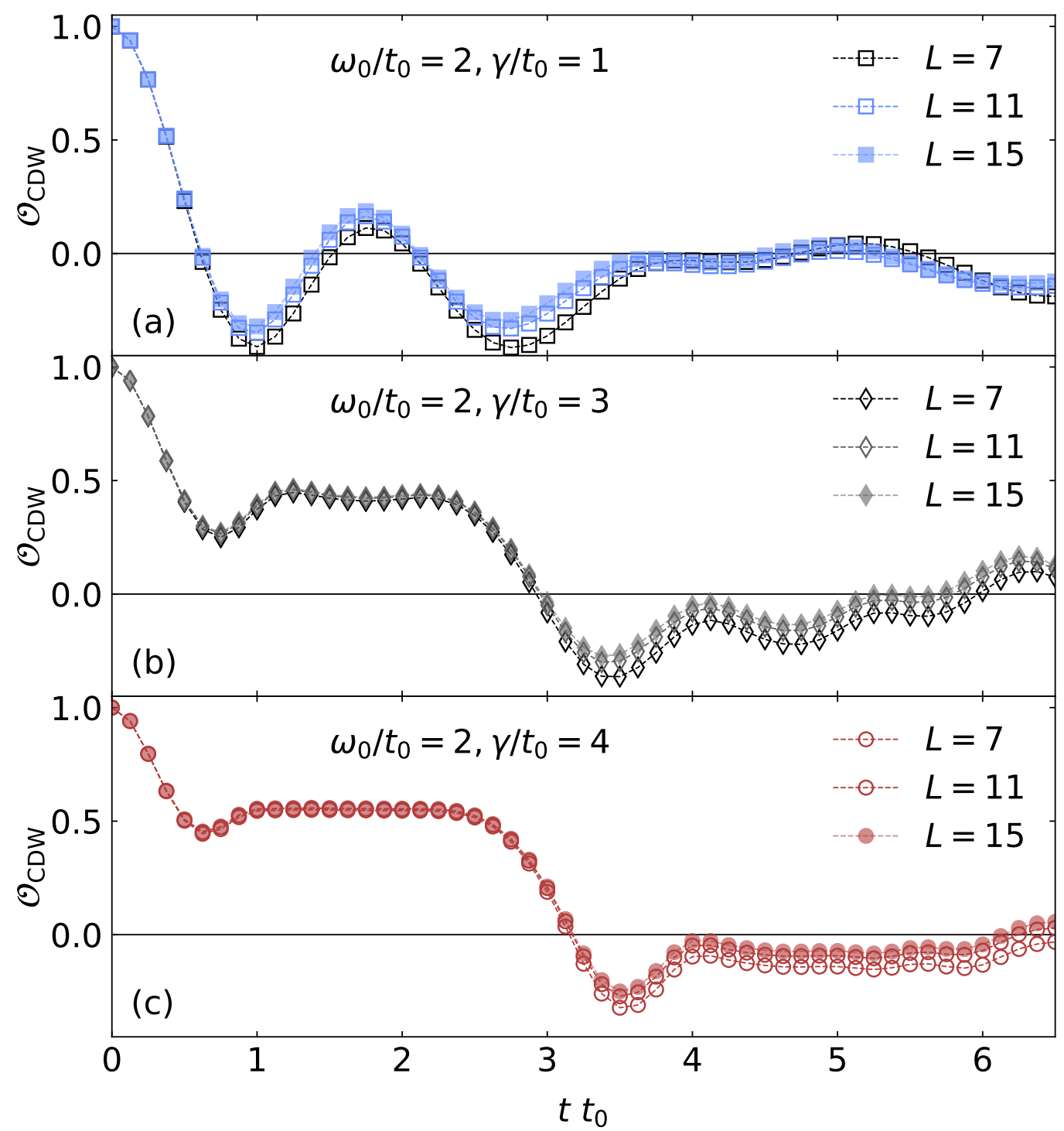

Figure A.1: Bare CDW melting: order parameter $\mathcal{O}_{\mathrm{CDW}}$ as a function of time for different system sizes $\mathrm{L}=7,11,15$. (a) $\omega_{0} / t_{0}=2, \gamma / t_{0}=1$, and $M_{\mathrm{ph}}=15$. (b) $\omega_{0} / t_{0}=2, \gamma / t_{0}=3$, and $M_{\mathrm{ph}}=30$. (c) $\omega_{0} / t_{0}=2, \gamma / t_{0}=4$, and $M_{\mathrm{ph}}=40$. For clarity, only every fifth data point computed with TEBDLBO is shown.

[Fig. A.3(b)] and only very small finite-size effects are visible.

To summarize, the comparisons in Figs. A.1, A.2, and A.3 establish the robustness of the phenomena discussed in Ch. 8 against finite-size effects. 


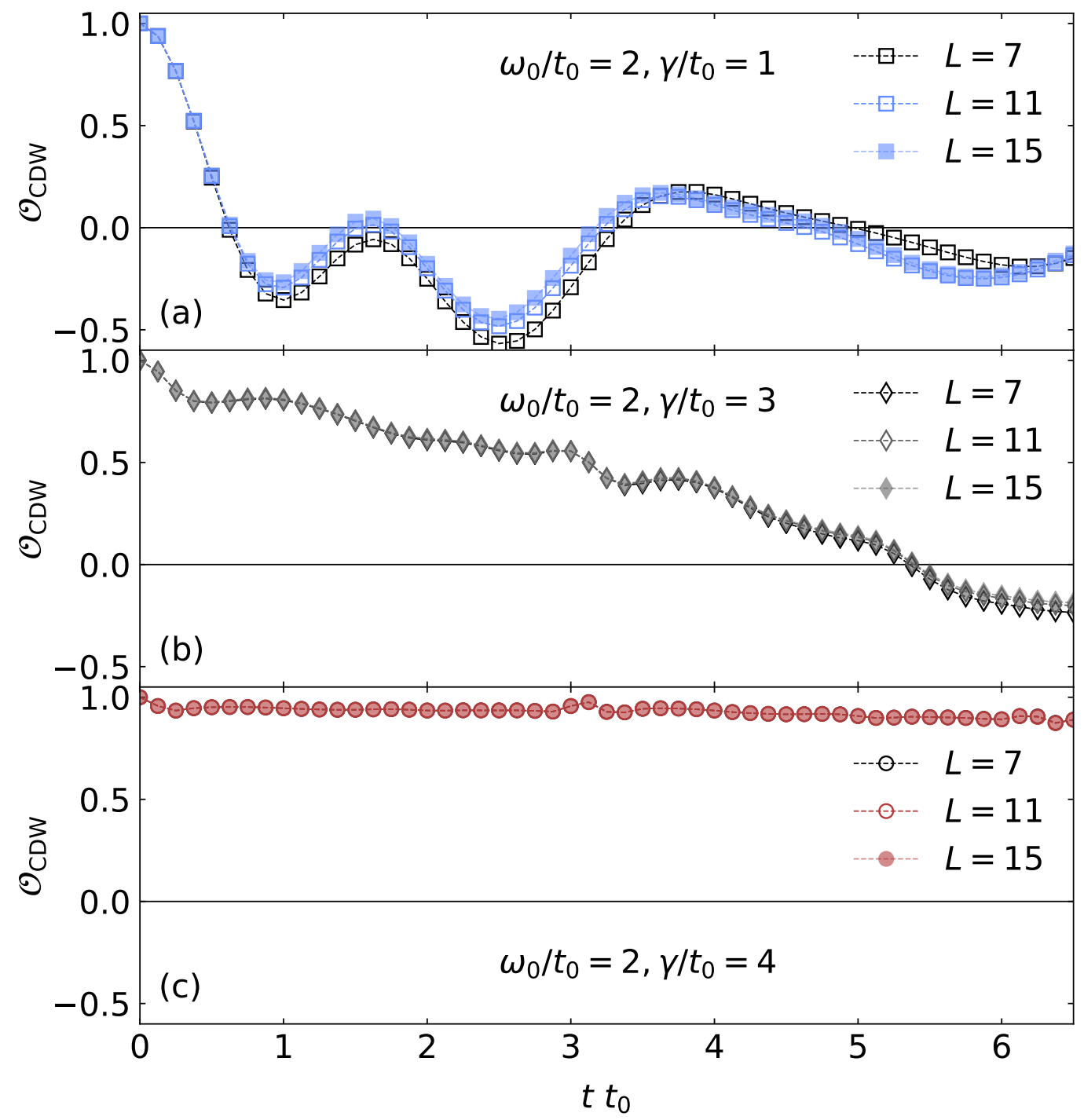

Figure A.2: Dressed CDW melting: order parameter $\mathcal{O}_{\mathrm{CDW}}$ as a function of time for different system sizes $\mathrm{L}=7,11,15$. (a) $\omega_{0} / t_{0}=2, \gamma / t_{0}=1$, and $M_{\mathrm{ph}}=15$. (b) $\omega_{0} / t_{0}=2, \gamma / t_{0}=3$, and $M_{\mathrm{ph}}=30$. (c) $\omega_{0} / t_{0}=2$, $\gamma / t_{0}=4$, and $M_{\mathrm{ph}}=40$. For clarity, only every fifth data point computed with TEBD-LBO is shown. 


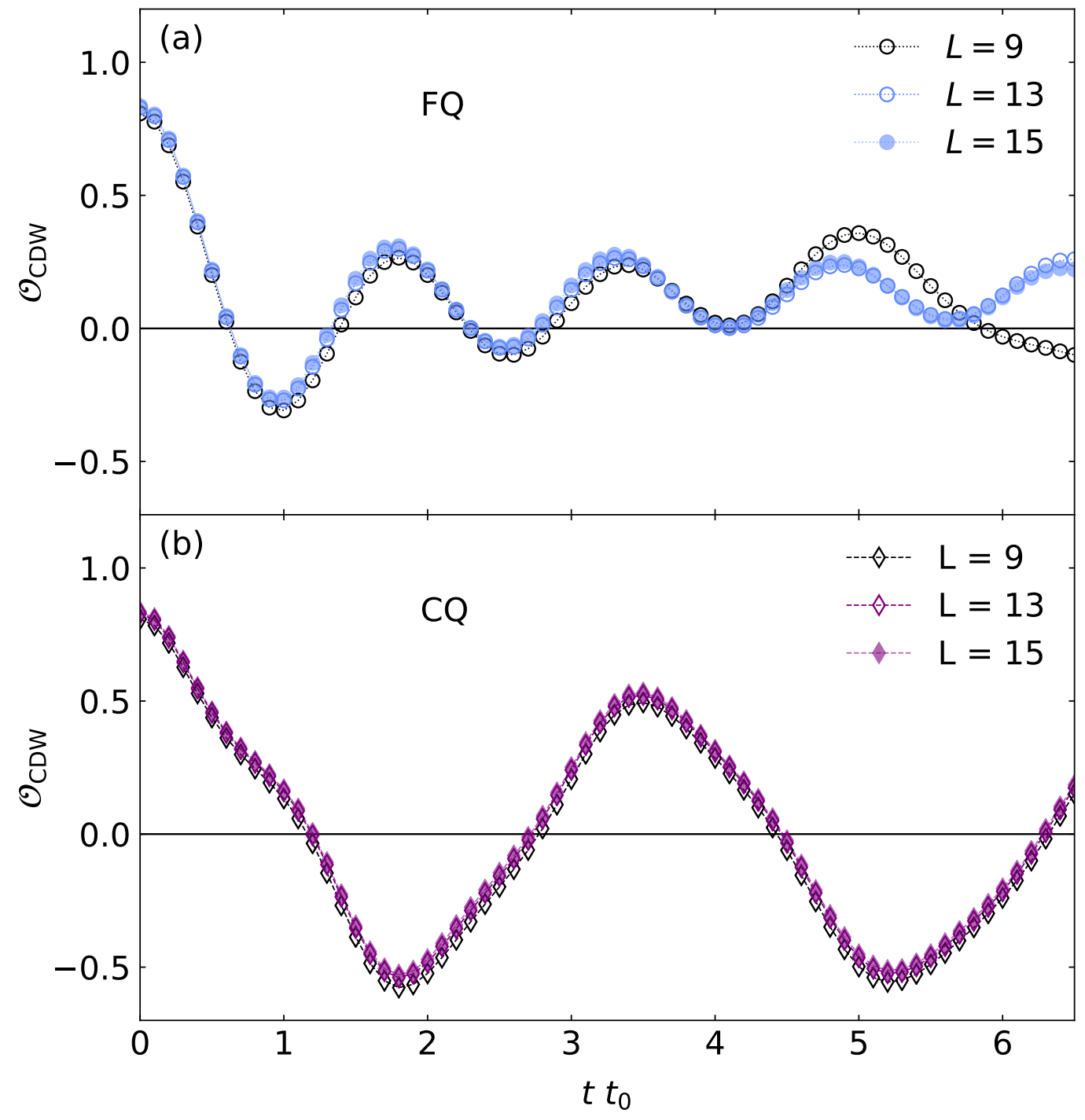

Figure A.3: Quench from CDW phase to metallic phase: order parameter $\mathcal{O}_{\mathrm{CDW}}$ as a function of time for different system sizes $\mathrm{L}=9,13,15$. (a) Frequency quench: $\omega_{0} / t_{0}=2, \gamma / t_{0}=4$ to $\omega_{0} / t_{0}=0.1, \gamma / t_{0}=0.2$. (b) Coupling quench: $\omega_{0} / t_{0}=2, \gamma / t_{0}=4$ to $\omega_{0} / t_{0}=2, \gamma / t_{0}=1$. For clarity, only every fourth data point computed with TEBD-LBO is shown. 


\section{Bibliography}

[1] N. W. Ashcroft and N. D. Mermin. Solid State Physics. W. B. Saunders Company, 1976.

[2] G. Giuliani and G. Vignale. Quantum theory of the electron liquid. Cambridge university press, 2005.

[3] D. Meschede. Gerthsen physik. Springer-Verlag, 2010.

[4] M. Born and K. Huang. Dynamical theory of crystal lattices. Clarendon press, 1954.

[5] P. E. Blöchl. $\Phi S X$ : Advanced Topics of Theoretical Physics I: Introduction to Solid-State Theory (Lecture Notes). http://www2.pt. tu-clausthal.de/atp/phisx.html (visited 18/05/2020).

[6] D. Emin. Polarons. Cambridge University Press, 2012.

[7] G. Grüner. The dynamics of charge-density waves. Rev. Mod. Phys., 60:1129-1181, Oct 1988.

[8] J. Bardeen, L. N. Cooper, and J. R. Schrieffer. Theory of superconductivity. Phys. Rev., 108:1175-1204, Dec 1957.

[9] J. Orenstein. Ultrafast spectroscopy of quantum materials. Physics Today, 65(9):44, 2012.

[10] C. Giannetti, M. Capone, D. Fausti, M. Fabrizio, F. Parmigiani, and D. Mihailovic. Ultrafast optical spectroscopy of strongly correlated materials and high-temperature superconductors: a non-equilibrium approach. Adv. Phys., 65(2):58-238, 2016.

[11] W. Hu, S. Kaiser, D. Nicoletti, C. R. Hunt, I. Gierz, M. C. Hoffmann, M. Le Tacon, T. Loew, B. Keimer, and A. Cavalleri. Optically enhanced coherent transport in $\mathrm{YBa}_{2} \mathrm{Cu}_{3} \mathrm{O}_{6.5}$ by ultrafast redistribution of interlayer coupling. Nat. Mater., 13(7):705, 2014. 
[12] S. Kaiser, C. R. Hunt, D. Nicoletti, W. Hu, I. Gierz, H. Y. Liu, M. Le Tacon, T. Loew, D. Haug, B. Keimer, and A. Cavalleri. Optically induced coherent transport far above $T_{c}$ in underdoped $\mathrm{YBa}_{2} \mathrm{Cu}_{3} \mathrm{O}_{6+\delta}$. Phys. Rev. B, 89:184516, May 2014.

[13] F. Schmitt, P. S. Kirchmann, U. Bovensiepen, R. G. Moore, L. Rettig, M. Krenz, J.-H. Chu, N. Ru, L. Perfetti, D. H. Lu, M. Wolf, I. R. Fisher, and Z.-X. Shen. Transient electronic structure and melting of a charge density wave in $\mathrm{TbTe}_{3}$. Science, 321(5896):1649-1652, 2008.

[14] A. Tomeljak, H. Schäfer, D. Städter, M. Beyer, K. Biljakovic, and J. Demsar. Dynamics of photoinduced charge-density-wave to metal phase transition in $\mathrm{K}_{0.3} \mathrm{MoO}_{3}$. Phys. Rev. Lett., 102:066404, Feb 2009.

[15] Y. Zhang, X. Shi, M. Guan, W. You, Y. Zhong, T. R. Kafle, Y. Huang, H. Ding, M. Bauer, K. Rossnagel, S. Meng, H. C. Kapteyn, and M. M. Murnane. Creation of a novel inverted charge density wave state. arXiv:2011.07623, Nov 2020.

[16] L. Stojchevska, I. Vaskivskyi, T. Mertelj, P. Kusar, D. Svetin, S. Brazovskii, and D. Mihailovic. Ultrafast switching to a stable hidden quantum state in an electronic crystal. Science, 344(6180):177-180, 2014.

[17] H. Okamoto, T. Miyagoe, K. Kobayashi, H. Uemura, H. Nishioka, H. Matsuzaki, A. Sawa, and Y. Tokura. Photoinduced transition from Mott insulator to metal in the undoped cuprates $\mathrm{Nd}_{2} \mathrm{CuO}_{4}$ and $\mathrm{La}_{2} \mathrm{CuO}_{4}$. Phys. Rev. B, 83:125102, Mar 2011.

[18] J. G. Horstmann, H. Böckmann, B. Wit, F. Kurtz, G. Storeck, and C. Ropers. Coherent control of a surface structural phase transition. Nature, 583(7815):232-236, 2020.

[19] T. Holstein. Studies of polaron motion: Part I. the molecular-crystal model. Ann. Phys. (N. Y.), 8(3):325 - 342, 1959.

[20] T. Holstein. Studies of polaron motion: Part II. the "small" polaron. Ann. Phys. (N. Y.), 8(3):343 - 389, 1959.

[21] O. S. Barišić and S. Barišić. Phase diagram of the Holstein polaron in one dimension. Eur. Phys. J. B, 64(1):1-18, Jul 2008.

[22] J. E. Hirsch and E. Fradkin. Phase diagram of one-dimensional electron-phonon systems. ii. the molecular-crystal model. Phys. Rev. B, 27:4302-4316, Apr 1983. 
[23] R. J. Bursill, R. H. McKenzie, and C. J. Hamer. Phase diagram of the one-dimensional Holstein model of spinless fermions. Phys. Rev. Lett., 80:5607-5610, Jun 1998.

[24] C. E. Creffield, G. Sangiovanni, and M. Capone. Phonon softening and dispersion in the 1D Holstein model of spinless fermions. Eur. Phys. J. B, 44(2):175-181, Mar 2005.

[25] L. G. Caron and C. Bourbonnais. Two-cutoff renormalization and quantum versus classical aspects for the one-dimensional electronphonon system. Phys. Rev. B, 29:4230-4241, Apr 1984.

[26] H. Fehske, H. Röder, G. Wellein, and A. Mistriotis. Hole-polaron formation in the two-dimensional Holstein $t-J$ model: A variational Lanczos study. Phys. Rev. B, 51:16582-16593, Jun 1995.

[27] P. E. Kornilovitch. Continuous-time quantum Monte Carlo algorithm for the lattice polaron. Phys. Rev. Lett., 81:5382-5385, Dec 1998.

[28] E. Jeckelmann and S. R. White. Density-matrix renormalization-group study of the polaron problem in the Holstein model. Phys. Rev. B, 57:6376-6385, Mar 1998.

[29] C. Zhang, E. Jeckelmann, and S. R. White. Density matrix approach to local Hilbert space reduction. Phys. Rev. Lett., 80:2661-2664, Mar 1998 .

[30] J. Bonča, S. A. Trugman, and I. Batistić. Holstein polaron. Phys. Rev. B, 60:1633-1642, Jul 1999.

[31] M. Hohenadler, H. G. Evertz, and W. von der Linden. Quantum Monte Carlo and variational approaches to the Holstein model. Phys. Rev. B, 69:024301, Jan 2004

[32] M. Berciu. Green's function of a dressed particle. Phys. Rev. Lett., 97:036402, Jul 2006.

[33] E. Jeckelmann and H. Fehske. Exact numerical methods for electronphonon problems. Riv. Nuovo Cimento, 30:259-292, 2007.

[34] G. L. Goodvin, A. S. Mishchenko, and M. Berciu. Optical conductivity of the Holstein polaron. Phys. Rev. Lett., 107:076403, Aug 2011. 
[35] M. Weber, F. F. Assaad, and M. Hohenadler. Continuous-time quantum Monte Carlo for fermion-boson lattice models: Improved bosonic estimators and application to the Holstein model. Phys. Rev. B, 94:245138, Dec 2016.

[36] Y. Murakami, N. Tsuji, M. Eckstein, and P. Werner. Nonequilibrium steady states and transient dynamics of conventional superconductors under phonon driving. Phys. Rev. B, 96:045125, Jul 2017.

[37] T. Köhler, J. Stolpp, and S. Paeckel. Efficient and flexible approach to simulate low-dimensional quantum lattice models with large local Hilbert spaces. SciPost Phys., 10:58, 2021.

[38] J. Stolpp, T. Köhler, S. R. Manmana, E. Jeckelmann, F. HeidrichMeisner, and S. Paeckel. Comparative study of state-of-the-art matrixproduct-state methods for lattice models with large local Hilbert spaces. arXiv:2011.07412, Nov 2020.

[39] A. W. Sandvik. Computational studies of quantum spin systems. In AIP Conference Proceedings, volume 1297, pages 135-338. American Institute of Physics, 2010.

[40] S. R. White. Density matrix formulation for quantum renormalization groups. Phys. Rev. Lett., 69:2863-2866, Nov 1992.

[41] G. Vidal. Efficient classical simulation of slightly entangled quantum computations. Phys. Rev. Lett., 91:147902, Oct 2003.

[42] U. Schollwöck. The density-matrix renormalization group. Rev. Mod. Phys., 77:259-315, Apr 2005.

[43] R. J. Bursill. Density-matrix renormalization-group algorithm for quantum lattice systems with a large number of states per site. Phys. Rev. B, 60:1643-1649, Jul 1999.

[44] B. Friedman. Optimal phonon approach to the spin Peierls model with nonadiabatic spin-phonon coupling. Phys. Rev. B, 61:6701-6705, Mar 2000 .

[45] R. J. Bursill and W. Barford. Large-scale numerical investigation of excited states in poly(para-phenylene). Phys. Rev. B, 66:205112, Nov 2002 . 
[46] Chunli Zhang, Eric Jeckelmann, and Steven R. White. Dynamical properties of the one-dimensional Holstein model. Phys. Rev. B, 60:1409214104, Nov 1999.

[47] H. Wong and Z.-D. Chen. Density matrix renormalization group approach to the spin-boson model. Phys. Rev. B, 77:174305, May 2008.

[48] F. Dorfner. Numerical methods for strongly correlated many-body systems with bosonic degrees of freedom. doctoral thesis, LudwigMaximilians-Universität München, Feb 2017.

[49] C. Guo, A. Weichselbaum, J. von Delft, and M. Vojta. Critical and strong-coupling phases in one- and two-bath spin-boson models. Phys. Rev. Lett., 108:160401, Apr 2012.

[50] C. Hubig, I. P. McCulloch, U. Schollwöck, and F. A. Wolf. Strictly single-site dmrg algorithm with subspace expansion. Phys. Rev. B, 91:155115, Apr 2015.

[51] C. Brockt, F. Dorfner, L. Vidmar, F. Heidrich-Meisner, and E. Jeckelmann. Matrix-product-state method with a dynamical local basis optimization for bosonic systems out of equilibrium. Phys. Rev. B, 92:241106, Dec 2015.

[52] C. Brockt and E. Jeckelmann. Scattering of an electronic wave packet by a one-dimensional electron-phonon-coupled structure. Phys. Rev. B, 95:064309, Feb 2017.

[53] C. Brockt. Numerical study of the nonequilibrium dynamics of 1-D electron-phonon systems using a local basis optimization. doctoral thesis, Gottfried Wilhelm Leibniz Universität Hannover, Jan 2018.

[54] J. Stolpp, J. Herbrych, F. Dorfner, E. Dagotto, and F. HeidrichMeisner. Charge-density-wave melting in the one-dimensional Holstein model. Phys. Rev. B, 101:035134, Jan 2020.

[55] I. P. McCulloch. From density-matrix renormalization group to matrix product states. Journal of Statistical Mechanics: Theory and Experiment, 2007(10):P10014-P10014, Oct 2007.

[56] S. Singh, R. N. C. Pfeifer, and G. Vidal. Tensor network states and algorithms in the presence of a global $\mathrm{u}(1)$ symmetry. Phys. Rev. B, 83:115125, Mar 2011. 
[57] H. Hashimoto and S. Ishihara. Photoinduced charge-order melting dynamics in a one-dimensional interacting Holstein model. Phys. Rev. B, 96:035154, Jul 2017.

[58] B. Kloss, D. R. Reichman, and R. Tempelaar. Multiset matrix product state calculations reveal mobile Franck-Condon excitations under strong Holstein-type coupling. Phys. Rev. Lett., 123:126601, Sep 2019.

[59] J. M. Deutsch. Quantum statistical mechanics in a closed system. Phys. Rev. A, 43:2046-2049, Feb 1991.

[60] M. Srednicki. Chaos and quantum thermalization. Phys. Rev. E, 50:888-901, Aug 1994.

[61] M. Srednicki. The approach to thermal equilibrium in quantized chaotic systems. Journal of Physics A: Mathematical and General, 32(7):11631175, Jan 1999.

[62] L. D'Alessio, Y. Kafri, A. Polkovnikov, and M. Rigol. From quantum chaos and eigenstate thermalization to statistical mechanics and thermodynamics. Advances in Physics, 65(3):239-362, 2016.

[63] M. Rigol, V. Dunjko, and M. Olshanii. Thermalization and its mechanism for generic isolated quantum systems. Nature, 452(7189):854-858, 2008 .

[64] S. Sorg, L. Vidmar, L. Pollet, and F. Heidrich-Meisner. Relaxation and thermalization in the one-dimensional Bose-Hubbard model: A case study for the interaction quantum quench from the atomic limit. Phys. Rev. A, 90:033606, Sep 2014.

[65] R. Mondaini, K. R. Fratus, M. Srednicki, and M. Rigol. Eigenstate thermalization in the two-dimensional transverse field Ising model. Phys. Rev. E, 93:032104, Mar 2016.

[66] R. Mondaini and M. Rigol. Eigenstate thermalization in the twodimensional transverse field Ising model. ii. off-diagonal matrix elements of observables. Phys. Rev. E, 96:012157, Jul 2017.

[67] T. Kinoshita, T. Wenger, and D. S Weiss. A quantum Newton's cradle. Nature, 440(7086):900-903, 2006. 
[68] M. Gring, M. Kuhnert, T. Langen, T. Kitagawa, B. Rauer, M. Schreitl, I. Mazets, D. Adu Smith, E. Demler, and J. Schmiedmayer. Relaxation and prethermalization in an isolated quantum system. Science, 337(6100):1318-1322, 2012.

[69] T. Langen, S. Erne, R. Geiger, B. Rauer, T. Schweigler, M. Kuhnert, W. Rohringer, I. E. Mazets, T. Gasenzer, and J. Schmiedmayer. Experimental observation of a generalized Gibbs ensemble. Science, 348(6231):207-211, 2015.

[70] D. Jansen, J. Stolpp, L. Vidmar, and F. Heidrich-Meisner. Eigenstate thermalization and quantum chaos in the Holstein polaron model. Phys. Rev. B, 99:155130, Apr 2019.

[71] L. F. Santos. Integrability of a disordered Heisenberg spin-1/2 chain. Journal of Physics A: Mathematical and General, 37(17):4723-4729, Apr 2004.

[72] O. S. Barišić, P. Prelovšek, A. Metavitsiadis, and X. Zotos. Incoherent transport induced by a single static impurity in a Heisenberg chain. Phys. Rev. B, 80:125118, Sep 2009.

[73] L. F. Santos and A. Mitra. Domain wall dynamics in integrable and chaotic spin-1/2 chains. Phys. Rev. E, 84:016206, Jul 2011.

[74] E. J. Torres-Herrera and L. F. Santos. Local quenches with global effects in interacting quantum systems. Phys. Rev. E, 89:062110, Jun 2014 .

[75] M. Brenes, E. Mascarenhas, M. Rigol, and J. Goold. High-temperature coherent transport in the xxz chain in the presence of an impurity. Phys. Rev. B, 98:235128, Dec 2018.

[76] M. Brenes, T. LeBlond, J. Goold, and M. Rigol. Eigenstate thermalization in a locally perturbed integrable system. Phys. Rev. Lett., 125:070605, Aug 2020.

[77] M. Lewenstein, A. Sanpera, V. Ahufinger, B. Damski, A. Sen(De), and U. Sen. Ultracold atomic gases in optical lattices: mimicking condensed matter physics and beyond. Advances in Physics, 56(2):243-379, 2007.

[78] I. Bloch, J. Dalibard, and W. Zwerger. Many-body physics with ultracold gases. Rev. Mod. Phys., 80:885-964, Jul 2008. 
[79] S. Scherg, T. Kohlert, J. Herbrych, J. Stolpp, P. Bordia, U. Schneider, F. Heidrich-Meisner, I. Bloch, and M. Aidelsburger. Nonequilibrium mass transport in the 1D Fermi-Hubbard model. Phys. Rev. Lett., 121:130402, Sep 2018.

[80] J. P. Ronzheimer, M. Schreiber, S. Braun, S. S. Hodgman, S. Langer, I. P. McCulloch, F. Heidrich-Meisner, I. Bloch, and U. Schneider. Expansion dynamics of interacting bosons in homogeneous lattices in one and two dimensions. Phys. Rev. Lett., 110:205301, May 2013.

[81] L. Xia, L. A. Zundel, J. Carrasquilla, A. Reinhard, J. M. Wilson, M. Rigol, and D. S. Weiss. Quantum distillation and confinement of vacancies in a doublon sea. Nature Physics, 11(4):316-320, 2015.

[82] F. Heidrich-Meisner, S. R. Manmana, M. Rigol, A. Muramatsu, A. E. Feiguin, and E. Dagotto. Quantum distillation: Dynamical generation of low-entropy states of strongly correlated fermions in an optical lattice. Phys. Rev. A, 80:041603, Oct 2009.

[83] D. Muth, D. Petrosyan, and M. Fleischhauer. Dynamics and evaporation of defects in Mott-insulating clusters of boson pairs. Phys. Rev. $A$, 85:013615, Jan 2012.

[84] J. Herbrych, A. E. Feiguin, E. Dagotto, and F. Heidrich-Meisner. Efficiency of fermionic quantum distillation. Phys. Rev. A, 96:033617, Sep 2017.

[85] J. M. Ziman. Principles of the Theory of Solids. Cambridge university press, 1972.

[86] P. Debye. Zur Theorie der spezifischen Wärmen. Annalen der Physik, 344(14):789-839, 1912.

[87] A. Einstein. Die Plancksche Theorie der Strahlung und die Theorie der spezifischen Wärme. Annalen der Physik, 327(1):180-190, 1907.

[88] L. D. Landau. Über die Bewegung der Elektronen in Kristallgitter. Phys. Z. Sowjetunion, 3:644-645, 1933.

[89] M. Tinkham. Introduction to Superconductivity. International series in pure and applied physics. McGraw Hill, 1996.

[90] L. N. Cooper. Bound electron pairs in a degenerate Fermi gas. Phys. Rev., 104:1189-1190, Nov 1956. 
[91] H. Fröhlich. Theory of the superconducting state. i. the ground state at the absolute zero of temperature. Phys. Rev., 79:845-856, Sep 1950.

[92] H. Fröhlich. Isotope effect in superconductivity. Proceedings of the Physical Society. Section A, 63(7):778-778, Jul 1950.

[93] E. Maxwell. Isotope effect in the superconductivity of mercury. Phys. Rev., 78:477-477, May 1950.

[94] C. A. Reynolds, B. Serin, W. H. Wright, and L. B. Nesbitt. Superconductivity of isotopes of mercury. Phys. Rev., 78:487-487, May 1950.

[95] P. A. Lee, N. Nagaosa, and X.G. Wen. Doping a Mott insulator: Physics of high-temperature superconductivity. Rev. Mod. Phys., 78:17-85, Jan 2006 .

[96] Q. Si, R. Yu, and E. Abrahams. High-temperature superconductivity in iron pnictides and chalcogenides. Nature Reviews Materials, 1(4):1-15, 2016.

[97] R. E. Peierls. Quantum Theory of Solids. Oxford University Press, 1955.

[98] M.J. Rice and S. Strässler. Theory of a quasi-one-dimensional bandconductor. Solid State Communications, 13(1):125 - 128, 1973.

[99] I. G. Lang and Yu. A. Firsov. Kinetic theory of semiconductors with low mobility. Sov. Phys. JETP, 16(5):1301, 1963.

[100] G. L. Goodvin, M. Berciu, and G. A. Sawatzky. Green's function of the Holstein polaron. Phys. Rev. B, 74:245104, Dec 2006.

[101] O. S. Barišić. Comment on "Green's function of a dressed particle". Phys. Rev. Lett., 98:209701, May 2007.

[102] M. Berciu. Berciu replies:. Phys. Rev. Lett., 98:209702, May 2007.

[103] M. Berciu and G. L. Goodvin. Systematic improvement of the momentum average approximation for the Green's function of a Holstein polaron. Phys. Rev. B, 76:165109, Oct 2007.

[104] R. H. McKenzie, C. J. Hamer, and D. W. Murray. Quantum Monte Carlo study of the one-dimensional Holstein model of spinless fermions. Phys. Rev. B, 53:9676-9687, Apr 1996. 
[105] T. Ohgoe and M. Imada. Variational Monte Carlo method for electronphonon coupled systems. Phys. Rev. B, 89:195139, May 2014.

[106] F. Dorfner, L. Vidmar, C. Brockt, E. Jeckelmann, and F. HeidrichMeisner. Real-time decay of a highly excited charge carrier in the one-dimensional Holstein model. Phys. Rev. B, 91:104302, Mar 2015.

[107] F. Dorfner and F. Heidrich-Meisner. Properties of the single-site reduced density matrix in the Bose-Bose resonance model in the ground state and in quantum quenches. Phys. Rev. A, 93:063624, Jun 2016.

[108] D. Jansen, J. Bonča, and F. Heidrich-Meisner. Finite-temperature density-matrix renormalization group method for electron-phonon systems: Thermodynamics and Holstein-polaron spectral functions. Phys. Rev. B, 102:165155, Oct 2020 .

[109] E. Jeckelmann, C. Zhang, and S. R. White. Metal-insulator transition in the one-dimensional Holstein model at half filling. Phys. Rev. B, 60:7950-7955, Sep 1999.

[110] S. Östlund and S. Rommer. Thermodynamic limit of density matrix renormalization. Phys. Rev. Lett., 75:3537-3540, Nov 1995.

[111] U. Schollwöck. The density-matrix renormalization group in the age of matrix product states. Annals of Physics, 326(1):96 - 192, 2011. January 2011 Special Issue.

[112] S. Paeckel, T. Köhler, A. Swoboda, S. R. Manmana, U. Schollwöck, and C. Hubig. Time-evolution methods for matrix-product states. Annals of Physics, 411:167998, 2019.

[113] F. Verstraete and J. I. Cirac. Matrix product states represent ground states faithfully. Phys. Rev. B, 73:094423, Mar 2006.

[114] M. B. Hastings. An area law for one-dimensional quantum systems. $J$. Stat. Mech.: Theory Exp., 2007(08):P08024, 2007.

[115] J. Eisert, M. Cramer, and M. B. Plenio. Colloquium: Area laws for the entanglement entropy. Rev. Mod. Phys., 82:277-306, Feb 2010.

[116] N. Laflorencie. Quantum entanglement in condensed matter systems. Phys. Rep., 646:1 - 59, 2016.

[117] G. Vidal. Efficient simulation of one-dimensional quantum many-body systems. Phys. Rev. Lett., 93:040502, Jul 2004. 
[118] S. R. White. Density matrix renormalization group algorithms with a single center site. Phys. Rev. B, 72:180403, Nov 2005.

[119] A. Weiße and H. Fehske. Peierls instability and optical response in the one-dimensional half-filled Holstein model of spinless fermions. Phys. Rev. B, 58:13526-13533, Nov 1998.

[120] J. Bonča, T. Katrašnik, and S. A. Trugman. Mobile bipolaron. Phys. Rev. Lett., 84:3153-3156, Apr 2000.

[121] Z. Li, D. Baillie, C. Blois, and F. Marsiglio. Ground-state properties of the Holstein model near the adiabatic limit. Phys. Rev. B, 81:115114, Mar 2010.

[122] L. Vidmar, J. Bonča, and S. A. Trugman. Emergence of states in the phonon spectral function of the Holstein polaron below and above the one-phonon continuum. Phys. Rev. B, 82:104304, Sep 2010.

[123] L. Vidmar, J. Bonča, T. Tohyama, and S. Maekawa. Quantum dynamics of a driven correlated system coupled to phonons. Phys. Rev. Lett., 107:246404, Dec 2011.

[124] L. Vidmar, J. Bonča, M. Mierzejewski, P. Prelovšek, and S. A. Trugman. Nonequilibrium dynamics of the Holstein polaron driven by an external electric field. Phys. Rev. B, 83:134301, Apr 2011.

[125] D. Golež, J. Bonča, L. Vidmar, and S. A. Trugman. Relaxation dynamics of the Holstein polaron. Phys. Rev. Lett., 109:236402, Dec 2012.

[126] D. Golež, J. Bonča, and L. Vidmar. Dissociation of a Hubbard-Holstein bipolaron driven away from equilibrium by a constant electric field. Phys. Rev. B, 85:144304, Apr 2012.

[127] C. J. Chandler and F. Marsiglio. Extended versus standard Holstein model: Results in two and three dimensions. Phys. Rev. B, 90:125131, Sep 2014.

[128] W. Barford, R. J. Bursill, and M. Y. Lavrentiev. Breakdown of the adiabatic approximation in trans-polyacetylene. Phys. Rev. B, 65:075107, Jan 2002.

[129] F. A. Y. N. Schröder and A. W. Chin. Simulating open quantum dynamics with time-dependent variational matrix product states: Towards microscopic correlation of environment dynamics and reduced system evolution. Phys. Rev. B, 93:075105, Feb 2016. 
[130] S. Paeckel. Topological and non-equilibrium superconductivity in lowdimensional strongly correlated quantum systems. doctoral thesis, Georg-August-Universität Göttingen, Feb 2020.

[131] F. Verstraete, J. J. García-Ripoll, and J. I. Cirac. Matrix product density operators: Simulation of finite-temperature and dissipative systems. Phys. Rev. Lett., 93:207204, Nov 2004.

[132] A. E. Feiguin and S. R. White. Finite-temperature density matrix renormalization using an enlarged Hilbert space. Phys. Rev. B, 72:220401, Dec 2005.

[133] S. Paeckel and T. Köhler. Ludwig-Maximilians-Universität München, Uppsala University. personal communication.

[134] M. Tezuka, R. Arita, and H. Aoki. Phase diagram for the onedimensional Hubbard-Holstein model: A density-matrix renormalization group study. Phys. Rev. B, 76:155114, Oct 2007.

[135] H. Fehske, G. Hager, and E. Jeckelmann. Metallicity in the half-filled Holstein-Hubbard model. EPL (Europhysics Letters), 84(5):57001, Nov 2008 .

[136] E. Jeckelmann. Gottfried Wilhelm Leibniz Universität Hannover. personal communication.

[137] J. Herbrych. Wrocław University of Science and Technology. personal communication.

[138] C. Hubig, J. Haegeman, and U. Schollwöck. Error estimates for extrapolations with matrix-product states. Phys. Rev. B, 97:045125, Jan 2018 .

[139] H. Ehrke, R. I. Tobey, S. Wall, S. A. Cavill, M. Först, V. Khanna, Th. Garl, N. Stojanovic, D. Prabhakaran, A. T. Boothroyd, M. Gensch, A. Mirone, P. Reutler, A. Revcolevschi, S. S. Dhesi, and A. Cavalleri. Photoinduced melting of antiferromagnetic order in $\mathrm{La}_{0.5} \mathrm{Sr}_{1.5} \mathrm{MnO}_{4}$ measured using ultrafast resonant soft x-ray diffraction. Phys. Rev. Lett., 106:217401, May 2011.

[140] S. R. Manmana, A. Muramatsu, and R. M. Noack. Time evolution of one-dimensional quantum many body systems. AIP Conference Proceedings, 789(1):269-278, 2005. 
[141] P. Barmettler, M. Punk, V. Gritsev, E. Demler, and E. Altman. Relaxation of antiferromagnetic order in spin- $1 / 2$ chains following a quantum quench. Phys. Rev. Lett., 102:130603, Apr 2009.

[142] H. Matsueda, S. Sota, T. Tohyama, and S. Maekawa. Relaxation dynamics of photocarriers in one-dimensional mott insulators coupled to phonons. Journal of the Physical Society of Japan, 81(1):013701, 2011.

[143] J. J. Mendoza-Arenas, D. F. Rojas-Gamboa, M. B. Plenio, and J. Prior. Exciton transport enhancement across quantum Su-Schrieffer-Heeger lattices with quartic nonlinearity. Phys. Rev. B, 100:104307, Sep 2019.

[144] J. M. Deutsch. Eigenstate thermalization hypothesis. Reports on Progress in Physics, 81(8):082001, Jul 2018.

[145] Joel L Lebowitz and Oliver Penrose. Modern ergodic theory. Physics Today, 26(2):23-29, 1973.

[146] O. Bohigas, M. J. Giannoni, and C. Schmit. Characterization of chaotic quantum spectra and universality of level fluctuation laws. Phys. Rev. Lett., 52:1-4, Jan 1984.

[147] R. Steinigeweg, J. Herbrych, and P. Prelovšek. Eigenstate thermalization within isolated spin-chain systems. Phys. Rev. E, 87:012118, Jan 2013 .

[148] W. Beugeling, R. Moessner, and Masudul Haque. Finite-size scaling of eigenstate thermalization. Phys. Rev. E, 89:042112, Apr 2014.

[149] W. Beugeling, R. Moessner, and M. Haque. Off-diagonal matrix elements of local operators in many-body quantum systems. Phys. Rev. E, 91:012144, Jan 2015.

[150] M. Srednicki. Thermal fluctuations in quantized chaotic systems. Journal of Physics A: Mathematical and General, 29(4):L75-L79, Feb 1996.

[151] M. Brenes, J. Goold, and M. Rigol. Low-frequency behavior of offdiagonal matrix elements in the integrable xxz chain and in a locally perturbed quantum-chaotic xxz chain. Phys. Rev. B, 102:075127, Aug 2020 .

[152] C. Schönle, D. Jansen, F. Heidrich-Meisner, and L. Vidmar. Eigenstate thermalization hypothesis through the lens of autocorrelation functions. arXiv:2011.13958, Nov 2020. 
[153] J. R. Garrison and T. Grover. Does a single eigenstate encode the full hamiltonian? Phys. Rev. X, 8:021026, Apr 2018.

[154] L. F. Santos and M. Rigol. Localization and the effects of symmetries in the thermalization properties of one-dimensional quantum systems. Phys. Rev. E, 82:031130, Sep 2010.

[155] T. A. Brody, J. Flores, J. B. French, P. A. Mello, A. Pandey, and S. S. M. Wong. Random-matrix physics: spectrum and strength fluctuations. Rev. Mod. Phys., 53:385-479, Jul 1981.

[156] L. F. Santos and M. Rigol. Onset of quantum chaos in one-dimensional bosonic and fermionic systems and its relation to thermalization. Phys. Rev. E, 81:036206, Mar 2010.

[157] V. Oganesyan and D. A. Huse. Localization of interacting fermions at high temperature. Phys. Rev. B, 75:155111, Apr 2007.

[158] Y. Y. Atas, E. Bogomolny, O. Giraud, and G. Roux. Distribution of the ratio of consecutive level spacings in random matrix ensembles. Phys. Rev. Lett., 110:084101, Feb 2013.

[159] H. Kim, T. N. Ikeda, and D. A. Huse. Testing whether all eigenstates obey the eigenstate thermalization hypothesis. Phys. Rev. E, 90:052105, Nov 2014.

[160] E. Khatami, G. Pupillo, M. Srednicki, and M. Rigol. Fluctuationdissipation theorem in an isolated system of quantum dipolar bosons after a quench. Phys. Rev. Lett., 111:050403, Jul 2013.

[161] J. Richter, J. Gemmer, and R. Steinigeweg. Impact of eigenstate thermalization on the route to equilibrium. Phys. Rev. E, 99:050104, May 2019 .

[162] T. Prosen. Ergodic properties of a generic nonintegrable quantum many-body system in the thermodynamic limit. Phys. Rev. E, 60:39493968, Oct 1999.

[163] L. Vidmar and M. Rigol. Entanglement entropy of eigenstates of quantum chaotic Hamiltonians. Phys. Rev. Lett., 119:220603, Nov 2017.

[164] J. Hubbard. Electron correlations in narrow energy bands. Proceedings of the Royal Society of London. Series A. Mathematical and Physical Sciences, 276(1365):238-257, 1963. 
[165] A. Görlitz, J. M. Vogels, A. E. Leanhardt, C. Raman, T. L. Gustavson, J. R. Abo-Shaeer, A. P. Chikkatur, S. Gupta, S. Inouye, T. Rosenband, and W. Ketterle. Realization of Bose-Einstein condensates in lower dimensions. Phys. Rev. Lett., 87:130402, Sep 2001.

[166] T. Giamarchi. Quantum physics in one dimension, volume 121. Clarendon press, 2003.

[167] A. Klümper. Integrability of quantum chains: Theory and applications to the spin-1/2 XXZ chain, pages 349-379. Springer Berlin Heidelberg, Berlin, Heidelberg, 2004.

[168] F. H. L. Essler, H. Frahm, F. Göhmann, A. Klümper, and V. E. Korepin. The One-Dimensional Hubbard Model. Cambridge University Press, 2005.

[169] M. A. Cazalilla, R. Citro, T. Giamarchi, E. Orignac, and M. Rigol. One dimensional bosons: From condensed matter systems to ultracold gases. Rev. Mod. Phys., 83:1405-1466, Dec 2011.

[170] B. Paredes, A. Widera, V. Murg, O. Mandel, S. Fölling, I. Cirac, G. V. Shlyapnikov, T. W. Hänsch, and I. Bloch. Tonks-Girardeau gas of ultracold atoms in an optical lattice. Nature, 429(6989):277-281, 2004.

[171] U. Schneider, L. Hackermüller, J. P. Ronzheimer, S. Will, S. Braun, T. Best, I. Bloch, E. Demler, S. Mandt, D. Rasch, and A. Rosch. Fermionic transport and out-of-equilibrium dynamics in a homogeneous Hubbard model with ultracold atoms. Nature Physics, 8(3):213$218,2012$.

[172] R. Rausch and M. Potthoff. Filling-dependent doublon dynamics in the one-dimensional Hubbard model. Phys. Rev. B, 95:045152, Jan 2017.

[173] A. Rosch, D. Rasch, B. Binz, and M. Vojta. Metastable superfluidity of repulsive fermionic atoms in optical lattices. Phys. Rev. Lett., 101:265301, Dec 2008.

[174] L. Vidmar, S. Langer, I. P. McCulloch, U. Schneider, U. Schollwöck, and F. Heidrich-Meisner. Sudden expansion of Mott insulators in one dimension. Phys. Rev. B, 88:235117, Dec 2013.

[175] T. Enss and J. Sirker. Light cone renormalization and quantum quenches in one-dimensional Hubbard models. New Journal of Physics, 14(2):023008, Feb 2012. 
[176] A. Bauer, F. Dorfner, and F. Heidrich-Meisner. Temporal decay of Néel order in the one-dimensional Fermi-Hubbard model. Phys. Rev. A, 91:053628, May 2015.

[177] P. Ehrenfest. Bemerkung über die angenäherte Gültigkeit der klassischen Mechanik innerhalb der Quantenmechanik. Zeitschrift für Physik, 45(7-8):455-457, 1927.

[178] N. Makri and W. H. Miller. Time-dependent self-consistent field (tdscf) approximation for a reaction coordinate coupled to a harmonic bath: Single and multiple configuration treatments. The Journal of Chemical Physics, 87(10):5781-5787, 1987. 


\section{Research data:}

The data shown throughout this thesis were stored using the GWDG project management service. In particular, the data can be found in the following git repositories:

Ch. 7:

ssh://git@projects.gwdg.de/holstein-dmrg3s-lbo-vs-dmrg-pseudosite.git (Jan Stolpp and Fabian Heidrich-Meisner have access)

Ch. 8:

ssh://git@projects.gwdg.de/project-as-a-service/stolpp/cdw-melting-in-the -holstein-model.git

(Jan Stolpp, Jacek Herbrych, and Fabian Heidrich-Meisner have access)

Ch. 10:

ssh://git@projects.gwdg.de/project-as-a-service/stolpp/qdist-fermions.git

(Jan Stolpp, Jacek Herbrych, and Fabian Heidrich-Meisner have access)

The data shown in Ch. 9 can be accessed via David Jansen in the git repository https://gitlab.physik.uni-muenchen.de/Jansen.David/HolsteinETH.

Furthermore, the DMRG code used by the author can be found at ssh://git@projects.gwdg.de/mps-libs.git (Jan Stolpp and Fabian HeidrichMeisner have access).

In addition, upon leaving the institute, the data will be permanently stored in the 10-Years-Archive by the IT-service of the Institut für Theoretische Physik, Georg-August-Universität Göttingen (Jürgen Holm) using the GWDG tape archive servers. 


\section{Acknowledgment:}

I thank Prof. Dr. Fabian Heidrich-Meisner for the excellent supervision of my research. The discussions and support from him were vital for the success of this dissertation and he gave me the opportunity to present my work on many conferences. Furthermore, he made a research stay at the University of Tennessee possible, which kickstarted my project on CDW melting. In this context, I have to thank Prof. Dr. Elbio Dagotto for his generous hospitality at the University of Tennessee and especially Dr. Jacek Herbrych for making my stay there a great experience. I also want to thank Eric Jeckelmann for being my second reviewer and also for hosting me at the University Hannover where Christoph Brockt helped me implement important parts of the TEBD-LBO method.

Next, I want to thank the people at the chair for theoretical nanophysics at the Ludwig-Maximilians-Universität München where I spent the first two years as a PhD-student before I moved to Göttingen.

At the CMT group in Göttingen I had a very warm welcome due to the academic and administrative staff. Especially the many coffee breaks with lots of interesting discussions, also about non physics related topics, were substantial for my well being during the PhD studies. Among others I thank Nils, Laura, Florian, Trisha, Lorenzo, Thomas, Sebastian, Kristof, Miroslav, David, Salvatore, Constantin, Kai, Michael and Eric.

My flat mates in Göttingen and especially Böschi made arriving and living there a most pleasant experience and I am thankful for all the distractions from my work.

Last but not least, I am deeply grateful for the support from my parents and family. They were always there for me when I needed them while at the same time they gave me the freedom to pursue my goals. 